\title{
Chiral Cyclopentadienyl Cobalt(III) Complexes Enable Highly Enantioselective 3d-Metal-Catalyzed C-H Functionalizations
}

\author{
Kristers Ozols, Yun-Suk Jang and Nicolai Cramer \\ Ecole Polytechnique Fédérale de Lausanne, Laboratory of Asymmetric Catalysis and Synthesis, \\ SB ISIC LCSA, BCH 4305, CH-1015 Lausanne, Switzerland \\ E-mail: nicolai.cramer@epfl.ch \\ Homepage: https://lcsa.epfl.ch/
}

\section{Supporting Information}

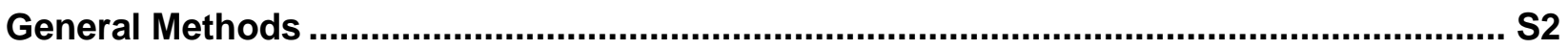

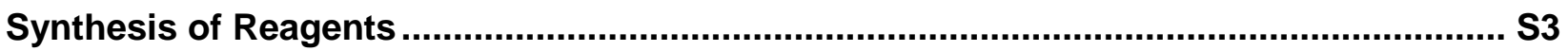

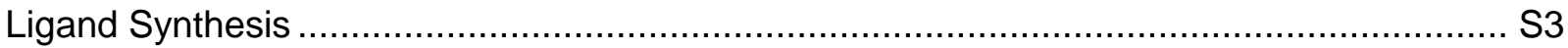

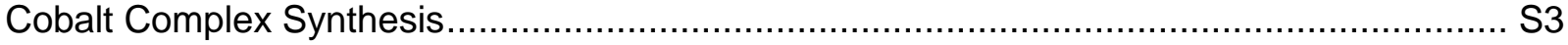

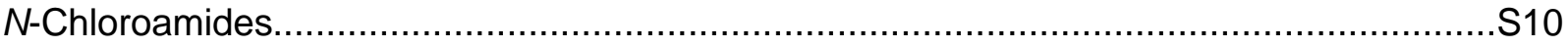

Styrenes, Acrylates and Other Vinyl Compounds ................................................... 11

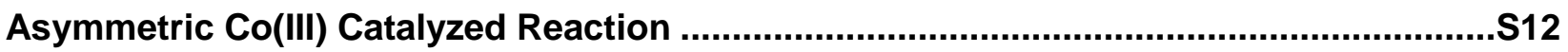

General Procedure for Optimization Table .................................................................. 12

General Procedure A (5.0 mol\% Cat. Loading) ............................................................

General Procedure B (5.0 mol\% Cat. Loading, Solid Reactant) ......................................S13

General Procedure C (10 mol\% Cat. Loading) ........................................................... 14

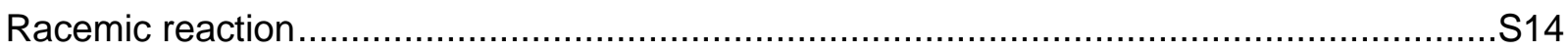

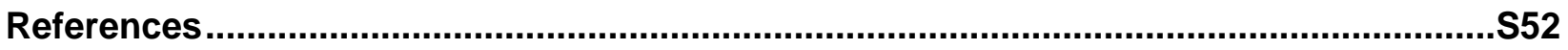

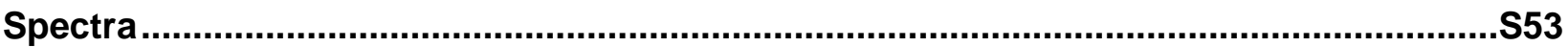




\section{General Methods}

All reactions were carried out under an atmosphere of nitrogen in oven-dried glassware with magnetic stirring, unless otherwise indicated. Dichloromethane and diethyl ether were purified by an Innovative Technology Solvent Delivery System degassed via freeze-pump-thaw technique and stored over $4 \AA$ molecular sieves in a nitrogen-filled glove box. TFE and HFIP were degassed via freeze-pump-thaw technique and stored over $3 \AA$ molecular sieves in a nitrogen-filled glove box. Chemicals were used as obtained from the suppliers unless otherwise indicated. Flash chromatography was performed with Silicycle silica gel 60 (0.040-0.063 $\mu \mathrm{m}$ grade). Analytical thinlayer chromatography was performed with commercial glass plates coated with $0.25 \mathrm{~mm}$ silica gel (E. Merck, Kieselgel 60 F254). Compounds were either visualized under UV-light at $254 \mathrm{~nm}$ or by dipping the plates in an aqueous potassium permanganate solution followed by heating. Proton nuclear magnetic resonance ( $\left.{ }^{1} \mathrm{H} \mathrm{NMR}\right)$ data was acquired on a Bruker AVANCE400 (400 MHz), Bruker DRX-400 (400 MHz), Bruker AVANCEIII-400 (400 MHz) or Bruker DRX-600 (600 MHz) spectrometer at $298 \mathrm{~K}$ unless otherwise noted. Chemical shifts $(\delta)$ are reported in parts per million (ppm) relative to incompletely deuterated $\mathrm{CDCl}_{3}(\mathrm{~s}, 7.26 \mathrm{ppm})$ or $\mathrm{C}_{6} \mathrm{D}_{6}(\mathrm{~s}, 7.16 \mathrm{ppm})$. Splitting patterns are designated as s, singlet; $d$, doublet; t, triplet; q, quartet; sept, septet; m, multiplet, br, broad. Proton-decoupled Carbon-13 nuclear magnetic resonance $\left({ }^{13} \mathrm{C}\left\{{ }^{1} \mathrm{H}\right\}\right.$ NMR) data were acquired on a Bruker AVANCE400 (101 MHz), Bruker DRX-400 (101 MHz), Bruker AVANCEIII$400(101 \mathrm{MHz})$ or Bruker DRX600 (151 MHz) spectrometer at $298 \mathrm{~K}$. Chemical shifts are reported in ppm relative to $\mathrm{CDCl}_{3}(77.16 \mathrm{ppm})$ or $\mathrm{C}_{6} \mathrm{D}_{6}(128.06 \mathrm{ppm})$. Proton-decoupled ${ }^{19} \mathrm{~F}$ nuclear magnetic resonance $\left({ }^{19} \mathrm{~F}\left\{{ }^{1} \mathrm{H}\right\} \mathrm{NMR}\right)$ data were acquired at $376 \mathrm{MHz}$ on a Bruker AVANCEIII-400 spectrometer at $298 \mathrm{~K}$. Infrared (IR) data were recorded on an Alpha-P Bruker FT-IR Spectrometer. Absorbance frequencies are reported in reciprocal centimeters $\left(\mathrm{cm}^{-1}\right)$. HRMS ESI measurements were performed by an Agilent LC-MS TOF and Xevo G2-S QTOF mass spectrometers. APPI-FTMS analyses were performed on a LTQ Orbitrap FTMS instrument. High resolution mass values are given in $\mathrm{m} / \mathrm{z}$. Optical rotations were measured on a Polartronic M polarimeter using a $10.0 \mathrm{~cm}$ cell with a Na $589 \mathrm{~nm}$ filter. X-ray analysis was performed by Dr. R. Scopelliti and Dr. F. Tirani at the EPF Lausanne. 


\section{Synthesis of Reagents}

\section{Ligand Synthesis}

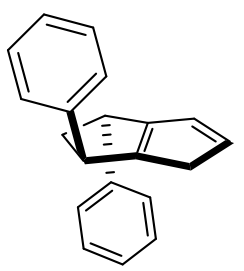

L1

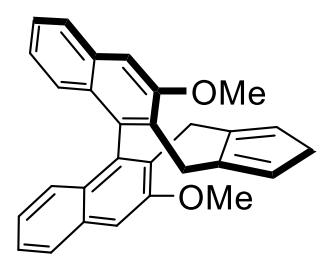

L2

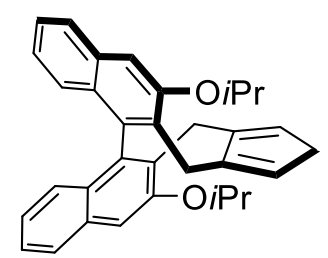

L3

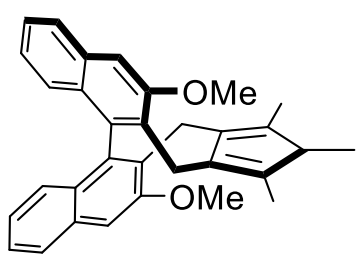

L4
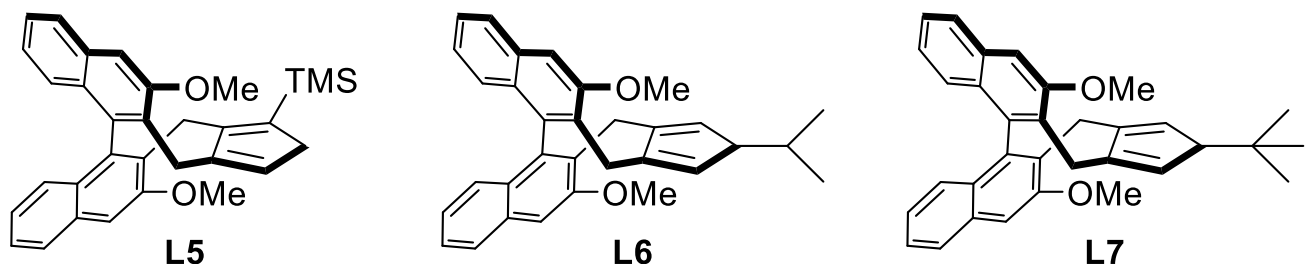

Figure 1: Ligands L1-L7 utilized for Co-complexation.

Ligands $\mathbf{L} 1^{1}, \mathbf{L} 2^{2}, \mathbf{L} 3^{2}, \mathbf{L} 4^{3}, \mathbf{L} 5^{4}, \mathbf{L} 6^{5}, \mathbf{L} 7^{5}$ were synthesized following the corresponding procedure.

\section{Cobalt Complex Synthesis}

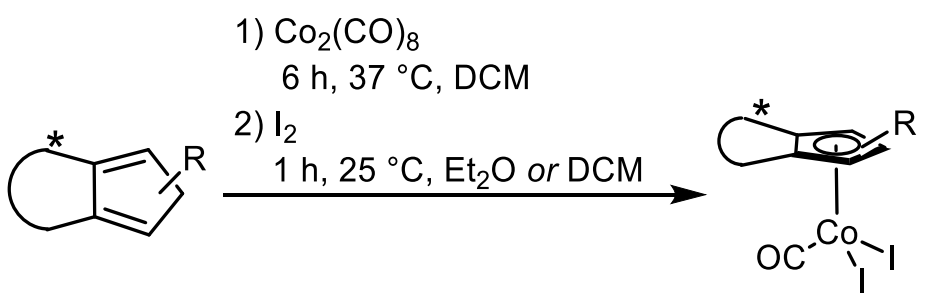

Scheme 1: Cobalt complexation.

The synthesis for complex $\mathbf{C o 7}$ was optimized by changing stoichiometry and reaction atmosphere (see table 1). The optimized conditions were then applied for the synthesis of Co1, Co2 and Co3. The syntheses of other cobalt complexes have not been repeated under the optimized conditions and are described as they were executed. 


\section{Cobalt complex $\mathrm{Cp}^{*} \mathrm{Co}(\mathrm{CO}) \mathbf{I}_{2}$}

$\mathrm{Cp}^{*} \mathrm{Co}(\mathrm{CO}) \mathrm{I}_{2}$ was prepared following a procedure reported by Matsunaga et al. ${ }^{6}$

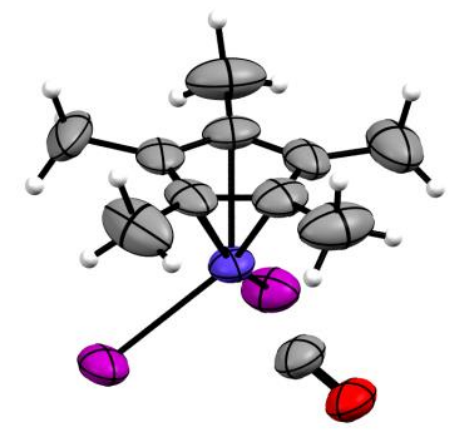

Figure 2: X-Ray Structure of $\mathbf{C p}^{*} \mathbf{C o}(\mathbf{C O}) \mathbf{I}_{\mathbf{2}}$ (CCDC: 1898853).

\section{Cobalt complex Co1}

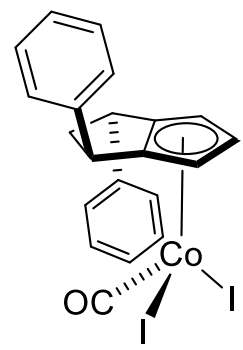

In an $\mathrm{N}_{2}$-filled glovebox, a flame dried Schlenk tube was charged with $\mathrm{Co}_{2}(\mathrm{CO})_{8}$ (61.3 mg, $170 \mu \mathrm{mol}, 1.10$ equiv.). A solution of $\mathbf{L} 1$ ( $40.0 \mathrm{mg}, 155 \mu \mathrm{mol}, 1.00$ equiv.) in dry DCM $(2.58 \mathrm{~mL})$ was added. CO gas was bubbled through the resulting solution for $5 \mathrm{~min}$ at $20^{\circ} \mathrm{C}$. The black reaction mixture was stirred at $37^{\circ} \mathrm{C}$ for 6 $\mathrm{h}$ under $\mathrm{CO}$ atmosphere. All volatiles were evaporated by Schlenk technique. A solution of iodine ( $47.2 \mathrm{mg}, 186 \mu \mathrm{mol}, 1.20$ equiv.) in distilled $\mathrm{Et}_{2} \mathrm{O}(2.58 \mathrm{~mL})$ was added to the black residue. The reaction vessel was connected to a Schlenk line to release carbon monoxide and the dark violet mixture was stirred at room temperature for $1 \mathrm{~h}$. All volatiles were evaporated and the residue was purified by column chromatography on silica gel (gradient: $\mathrm{DCM} /$ pentane $=0 \% \rightarrow 50 \%$ ). The dark violet fractions were collected and concentrated under reduced pressure to obtain $39.0 \mathrm{mg}(65.0 \mu \mathrm{mol}, 42 \%$ yield) of the desired product as a black solid. ${ }^{1} \mathrm{H}$ NMR $(600 \mathrm{MHz}) \delta=7.48-7.45(\mathrm{~m}, 4 \mathrm{H}), 7.42-7.38(\mathrm{~m}, 1 \mathrm{H}), 7.35(\mathrm{t}, J=7.4 \mathrm{~Hz}, 2 \mathrm{H}), 7.30(\mathrm{t}$, $J=7.4 \mathrm{~Hz}, 1 \mathrm{H}), 7.15(\mathrm{~d}, J=7.4 \mathrm{~Hz}, 2 \mathrm{H}), 6.17(\mathrm{t}, J=2.3 \mathrm{~Hz}, 1 \mathrm{H}), 5.73(\mathrm{~d}, J=2.3 \mathrm{~Hz}, 1 \mathrm{H}), 4.89(\mathrm{~d}$, $J=2.3 \mathrm{~Hz}, 1 \mathrm{H}$ ), $4.78(\mathrm{~d}, J=8.1 \mathrm{~Hz}, 1 \mathrm{H}$ ), $4.43(\mathrm{dd}, J=10.8,7.2 \mathrm{~Hz}, 1 \mathrm{H}$ ), 3.34 (ddd, $J=12.8,10.8$, $8.1 \mathrm{~Hz}, 1 \mathrm{H}), 2.72(\mathrm{dd}, J=12.8,7.8 \mathrm{~Hz}, 1 \mathrm{H}) \mathrm{ppm} ;{ }^{13} \mathrm{C}\left\{{ }^{1} \mathrm{H}\right\} \mathbf{N M R}(150 \mathrm{MHz}) \delta=195.0,141.4,138.2$, 129.3 (2C), 128.3, 127.7, 127.23, 127.19, 121.0, 118.6, 97.4, 81.7, 73.5, 44.2, 42.0, 41.2 ppm; IR (ATR): $\tilde{v}=3085,2924,2835,2062,1600,1493,1447,1367,1079,1030,842,752,698 \mathrm{~cm}^{-1}$;

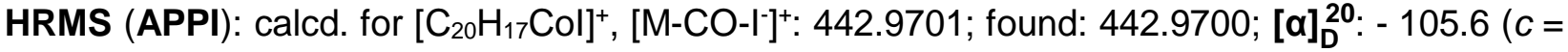
$\left.0.30, \mathrm{CHCl}_{3}\right)$. 


\section{Cobalt complex Co2}

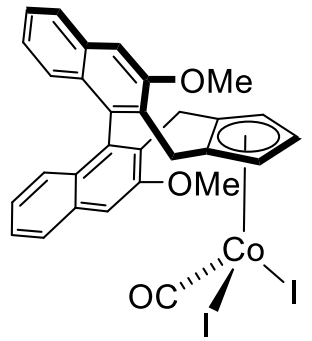

In an $\mathrm{N}_{2}$-filled glovebox, a flame dried Schlenk tube was charged with $\mathrm{Co}_{2}(\mathrm{CO})_{8}(48.9 \mathrm{mg}, 136.0 \mu \mathrm{mol}, 1.10$ equiv.). A solution of $\mathbf{L} 2(50.0 \mathrm{mg}$, $124 \mu \mathrm{mol}, 1.00$ equiv. $)$ in dry DCM $(2.00 \mathrm{~mL})$ was added. CO gas was bubbled through the resulting solution for $5 \mathrm{~min}$ at $20^{\circ} \mathrm{C}$. The black reaction mixture evaporated by Schlenk technique. A solution of iodine (37.6 mg, $148 \mu \mathrm{mol}, 1.20$ equiv.) in dry degassed $\mathrm{Et}_{2} \mathrm{O}(2.00 \mathrm{~mL})$ was added to the black residue. The reaction vessel was connected to a Schlenk line to release carbon monoxide and the dark violet mixture was stirred at room temperature for $1 \mathrm{~h}$. All volatiles were evaporated and the residue was purified by column chromatography on silica gel (gradient: DCM/pentane $=0 \% \rightarrow 50 \%$ ). The dark violet fractions were collected and concentrated under reduced pressure to obtain $84.0 \mathrm{mg}(113 \mu \mathrm{mol}, 91 \%$ yield $)$ of the desired product as a black solid.

${ }^{1} \mathrm{H}$ NMR $\left(600 \mathrm{MHz}, \mathrm{CDCl}_{3}\right) \delta=7.88(\mathrm{~d}, J=8.2 \mathrm{~Hz}, 1 \mathrm{H}), 7.81(\mathrm{~d}, J=8.2 \mathrm{~Hz}, 1 \mathrm{H}), 7.48-7.38(\mathrm{~m}$, $3 \mathrm{H}), 7.24(\mathrm{~s}, 1 \mathrm{H}), 7.15-7.07(\mathrm{~m}, 3 \mathrm{H}), 7.02(\mathrm{~d}, J=8.4 \mathrm{~Hz}, 1 \mathrm{H}), 5.85-5.81(\mathrm{~m}, 1 \mathrm{H}), 5.77-5.73$ (m, 1H), $5.48-5.42(\mathrm{~m}, 1 \mathrm{H}) 4.12(\mathrm{~s}, 3 \mathrm{H}), 4.06(\mathrm{~d}, J=16.2 \mathrm{~Hz}, 1 \mathrm{H}), 3.97(\mathrm{~s}, 3 \mathrm{H}), 3.90(\mathrm{~d}, J=14.1$ $\mathrm{Hz}, 1 \mathrm{H}), 3.60(\mathrm{~d}, J=14.1 \mathrm{~Hz}, 1 \mathrm{H}), 2.77(\mathrm{~d}, J=16.2 \mathrm{~Hz}, 1 \mathrm{H}) \mathrm{ppm} ;{ }^{13} \mathrm{C}\left\{{ }^{1} \mathrm{H}\right\} \mathrm{NMR}\left(150 \mathrm{MHz}, \mathrm{CDCl}_{3}\right)$ $\delta=196.8,155.7,154.8,138.1,136.9,134.2,133.9,127.22,127.19,127.11,127.07,127.0,126.9$, $126.8(3 \times \mathrm{C}), 124.5,124.3,123.7,106.28,106.26,104.4,103.4,94.8,90.7,85.4,55.8,55.4,26.7$, 24.7 ppm; IR (ATR): $\widetilde{v}=3062,2935,2061,1697,1503,1451,1238,1114,1019,907,730 \mathrm{~cm}^{-1}$; HRMS (APPI): calcd. for $\left[\mathrm{C}_{29} \mathrm{H}_{23} \mathrm{ColO}_{2}^{+}\right]^{+}, \quad$ [M-CO-l'] $]^{+}$:589.0069; found: 589.0068; $[\alpha]_{D}^{20}$ : $-91.2\left(c=0.30, \mathrm{CHCl}_{3}\right)$.

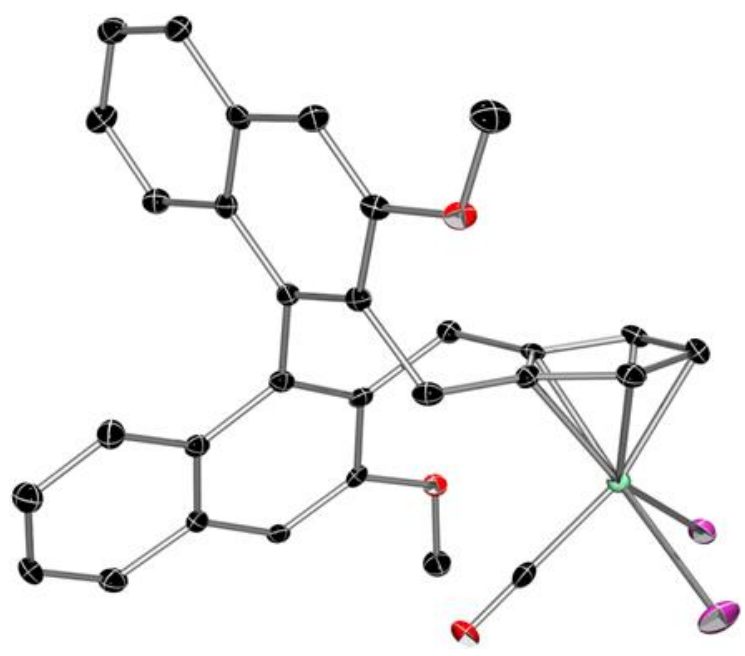

Figure 3: X-Ray Structure of Co2 (CCDC: 1898842). 


\section{$\underline{\text { Cobalt complex Co3 }}$}

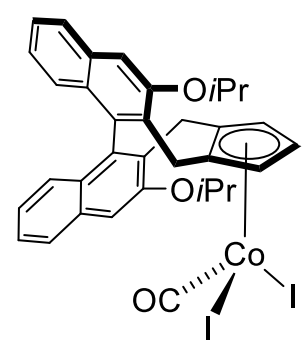

A flame-dried MW tube was charged with $\mathbf{L} 3(20.0 \mathrm{mg}, 43.0 \mu \mathrm{mol}, 1.00$ equiv.), $\mathrm{Co}_{2}(\mathrm{CO})_{8}(17.1 \mathrm{mg}, 48.0 \mu \mathrm{mol}, 1.10$ equiv.) and distilled $\mathrm{DCM}(720 \mu \mathrm{L})$ in an $\mathrm{N}_{2}$-filled glove box. The black solution was stirred at $40^{\circ} \mathrm{C}$ for $6 \mathrm{~h}$ in a sealed tube under nitrogen atmosphere. All volatiles were evaporated under a stream of nitrogen at room temperature. The black residue was dissolved with distilled $\mathrm{Et}_{2} \mathrm{O}(720 \mu \mathrm{L})$ and a solution of $\mathrm{I}_{2}(13.2 \mathrm{mg}, 52.0 \mu \mathrm{mol}, 1.20$ equiv. $)$ in distilled $\mathrm{Et}_{2} \mathrm{O}(1.00 \mathrm{~mL})$ was added to the Co-complex solution. The reaction vessel was connected to a Schlenk line to release carbon monoxide and the dark violet mixture was stirred at room temperature for $1 \mathrm{~h}$. Pentane $(2.00 \mathrm{~mL})$ was added to the crude mixture and the solution was purified by flash column chromatography on silica gel (gradient: DCM/pentane $=0 \% \rightarrow 50 \%$ ). The dark violet fractions were collected and concentrated under reduced pressure to obtain $26.0 \mathrm{mg}$ (32.0 $\mu \mathrm{mol}, 75 \%$ yield) of the desired product as a black solid. ${ }^{1} \mathrm{H}$ NMR $\left(400 \mathrm{MHz}, \mathrm{CDCl}_{3}\right) \delta=7.85$ (d, $J=8.2 \mathrm{~Hz}, 1 \mathrm{H}$ ), 7.75 (d, J = 8.2 Hz, 1H), $7.49-7.35$ (m, 3H), 7.20 (s, 1H), 7.10 (dtd, $J=8.0$, 6.7, $1.3 \mathrm{~Hz}, 2 \mathrm{H}), 7.01(\mathrm{~d}, J=8.5 \mathrm{~Hz}, 2 \mathrm{H}), 5.86$ (t, $J=2.2 \mathrm{~Hz}, 1 \mathrm{H}), 5.79$ (t, $J=2.6 \mathrm{~Hz}, 1 \mathrm{H}$ ), 5.51 (t, $J=2.2 \mathrm{~Hz}, 1 \mathrm{H}$ ), 4.92 (hept, $J=6.1 \mathrm{~Hz}, 1 \mathrm{H}$ ), 4.77 (hept, $J=6.1 \mathrm{~Hz}, 1 \mathrm{H}$ ), $4.12(\mathrm{~d}, J=16.2 \mathrm{~Hz}, 1 \mathrm{H}$ ), $3.88(\mathrm{~d}, J=13.7 \mathrm{~Hz}, 1 \mathrm{H}), 3.42(\mathrm{~d}, J=13.7 \mathrm{~Hz}, 1 \mathrm{H}), 2.74(\mathrm{~d}, J=16.2 \mathrm{~Hz}, 1 \mathrm{H}), 1.73(\mathrm{~d}, J=6.1 \mathrm{~Hz}$, $3 \mathrm{H}), 1.56(\mathrm{~d}, J=6.1 \mathrm{~Hz}, 3 \mathrm{H}), 1.50(\mathrm{~d}, J=6.1 \mathrm{~Hz}, 3 \mathrm{H}), 1.39(\mathrm{~d}, J=6.1 \mathrm{~Hz}, 3 \mathrm{H}) \mathrm{ppm} ;{ }^{13} \mathbf{C}\left\{{ }^{1} \mathrm{H}\right\} \mathbf{N M R}$ $\left(101 \mathrm{MHz}, \mathrm{CDCl}_{3}\right) \delta=196.5,154.2,152.6,138.5,137.0,134.3,134.0,127.3,127.1,127.0,126.9$ (four peaks), 126.8, 126.7, 124.4, 124.3, 124.1, 107.8, 107.5, 104.4, 101.0, 96.7, 93.4, 83.9, 71.2, 70.1, 26.3, 24.8, 22.5, 22.4, 22.3, 22.0 ppm; IR (ATR): 3061, 2974, 2931, 2870, 2061, 1617, 1593, 1436, 1384, 1373, 1292, 1085,908, 835, 730, $468 \mathrm{~cm}^{-1}$; HRMS (ESI): calcd. for $\left[\mathrm{C}_{33} \mathrm{H}_{31} \mathrm{ColO}_{2}\right]^{+}$, [M-CO-I] $]^{+}$: 645.0701; found: 645.0702 ; $\mathrm{mp}$ : a sample was heated over $410^{\circ} \mathrm{C}$, no change was observed; $[\alpha]_{D}^{20}:-29.5\left(c=1.00, \mathrm{CHCl}_{3}\right)$.

\section{Cobalt complex Co4}

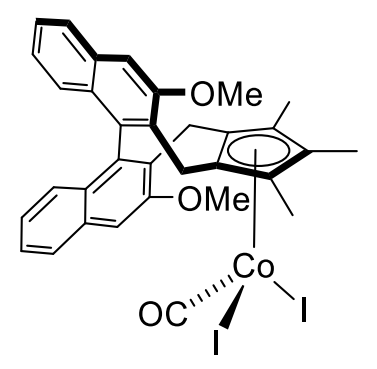

In an $\mathrm{N}_{2}$-filled glovebox, a flame-dried Schlenk tube was charged with $\mathrm{Co}_{2}(\mathrm{CO})_{8}(63.2 \mathrm{mg}, 185 \mu \mathrm{mol}, 0.55$ equiv.). A solution of $\mathbf{L} 4$ (150 mg, $336 \mu \mathrm{mol}, 1.00$ equiv. $)$ in dry DCM $(6.90 \mathrm{~mL})$ was added. The Schlenk tube was connected to a Schlenk line for $\mathrm{CO}$ evolution and stirred at $37^{\circ} \mathrm{C}$ for $6 \mathrm{~h}$. The volatiles were evaporated by Schlenk technique. A solution of 
iodine ( $46.9 \mathrm{mg}, 185 \mu \mathrm{mol}, 0.550$ equiv.) in dry degassed $\mathrm{Et}_{2} \mathrm{O}(4.60 \mathrm{~mL})$ was added to the black residue. The reaction vessel was connected to a Schlenk line to release carbon monoxide and the dark violet mixture was stirred at room temperature for $1 \mathrm{~h}$. All volatiles were evaporated and the residue was purified by column chromatography on silica gel (gradient: DCM/pentane $=0 \% \rightarrow$ $50 \%$ ). The dark violet fractions were collected and concentrated under reduced pressure to obtain $116 \mathrm{mg}$ ( $147 \mu \mathrm{mol}, 44 \%$ yield) of the desired product as a black solid. The analytical data match literature values. ${ }^{3}{ }^{1} \mathrm{H}$ NMR $\left(400 \mathrm{MHz}, \mathrm{CDCl}_{3}\right) \delta=7.86(\mathrm{~d}, J=8.2 \mathrm{~Hz}, 1 \mathrm{H}), 7.81(\mathrm{~d}, J=8.2 \mathrm{~Hz}, 1 \mathrm{H})$, $7.46-7.39(\mathrm{~m}, 2 \mathrm{H}), 7.36(\mathrm{~s}, 1 \mathrm{H}), 7.24(\mathrm{~s}, 1 \mathrm{H}), 7.16-7.05(\mathrm{~m}, 3 \mathrm{H}), 6.98(\mathrm{~d}, J=8.4 \mathrm{~Hz}, 1 \mathrm{H}), 4.12$ (s, 3H), $4.09(\mathrm{~d}, J=16.2 \mathrm{~Hz}, 1 \mathrm{H}), 3.98(\mathrm{~s}, 3 \mathrm{H}), 3.82(\mathrm{~d}, J=14.2 \mathrm{~Hz}, 1 \mathrm{H}), 3.68(\mathrm{~d}, J=14.2 \mathrm{~Hz}, 1 \mathrm{H})$, $2.64-2.57(\mathrm{~m}, 4 \mathrm{H}), 2.34(\mathrm{~s}, 3 \mathrm{H}), 2.24(\mathrm{~s}, 3 \mathrm{H}) \mathrm{ppm}$.

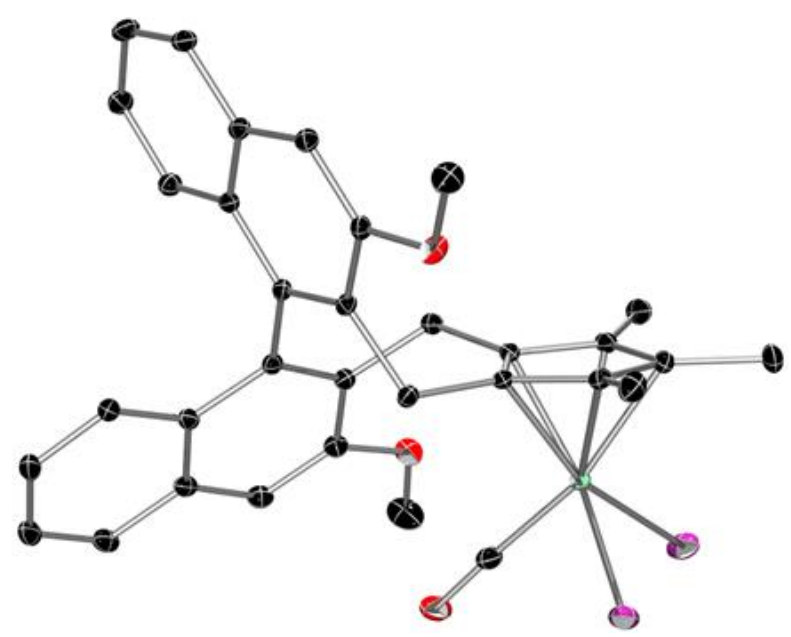

Figure 4: X-Ray Structure of Co4 (CCDC: 1898843).

\section{Cobalt complex Co5}

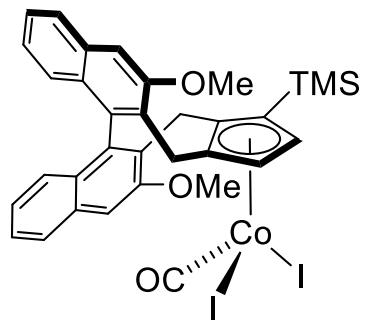

In an $\mathrm{N}_{2}$-filled glovebox, a flame dried Schlenk tube was charged with $\mathrm{Co}_{2}(\mathrm{CO})_{8}(22.0 \mathrm{mg}, 61.0 \mu \mathrm{mol}, 0.55$ equiv.). A solution of $\mathbf{L} 5(53.0 \mathrm{mg}$, $111 \mu \mathrm{mol}, 1.00$ equiv. $)$ in dry DCM $(2.30 \mathrm{~mL})$ was added. The Schlenk tube was connected to a Schlenk line for $\mathrm{CO}$ evolution and stirred at $37^{\circ} \mathrm{C}$ for $6 \mathrm{~h}$. The resulting solution was evaporated by Schlenk technique. A solution of iodine ( $15.5 \mathrm{mg}, 61.0 \mu \mathrm{mol}, 0.55$ equiv.) in dry degassed $\mathrm{Et}_{2} \mathrm{O}(1.50 \mathrm{~mL})$ was added to the solid black residue. The reaction vessel was connected to a Schlenk line to release carbon monoxide and the dark violet mixture was stirred at room temperature for $1 \mathrm{~h}$. All volatiles were evaporated and the product was purified by column chromatography on silica gel (gradient: $\mathrm{DCM} /$ pentane $=0 \% \rightarrow 50 \%$ ). The dark violet fractions ${ }^{*}$ were collected and concentrated under

*Some fractions also contained desilylated complex. 
reduced pressure to obtain $20.0 \mathrm{mg}(24.0 \mu \mathrm{mol}, 22 \%$ yield $)$ of the desired product as a black solid. ${ }^{1}$ H NMR $\left(600 \mathrm{MHz}, \mathrm{CDCl}_{3}\right) \delta=7.88(\mathrm{~d}, J=8.2 \mathrm{~Hz}, 1 \mathrm{H}), 7.79(\mathrm{~d}, J=7.8 \mathrm{~Hz}, 1 \mathrm{H}), 7.45$ (ddd, $J=$ 7.9, 6.9, $1.0 \mathrm{~Hz}, 1 \mathrm{H}), 7.43-7.40(\mathrm{~m}, 2 \mathrm{H}), 7.23(\mathrm{~s}, 1 \mathrm{H}), 7.14-7.07(\mathrm{~m}, 3 \mathrm{H}), 7.01$ (d, J = 8.3 Hz, $1 \mathrm{H}), 5.64(\mathrm{~d}, J=1.7 \mathrm{~Hz}, 1 \mathrm{H}), 5.61(\mathrm{~d}, J=1.7 \mathrm{~Hz}, 1 \mathrm{H}), 4.14(\mathrm{~d}, J=15.9 \mathrm{~Hz}, 1 \mathrm{H}), 4.11(\mathrm{~s}, 3 \mathrm{H}), 3.96$ (s, 3H) $3.85(\mathrm{~d}, J=13.8 \mathrm{~Hz}, 1 \mathrm{H}), 3.28(\mathrm{~d}, J=13.8 \mathrm{~Hz}, 1 \mathrm{H}), 2.73(\mathrm{~d}, J=15.9 \mathrm{~Hz}, 1 \mathrm{H}), 0.53(\mathrm{~s}, 9 \mathrm{H})$ ppm; ${ }^{13} \mathrm{C}\left\{{ }^{1} \mathrm{H}\right\}$ NMR $\left(150 \mathrm{MHz}, \mathrm{CDCl}_{3}\right) \delta=197.6,155.8,154.7,138.1,136.9,134.4,133.9,127.4$, 127.23, 127.21, 127.1, 127.0, 126.9 (3×C), 126.5, 124.64, 124.57, 124.3, 106.33, 106.27, 105.7, 102.3, 102.0, 101.1, 89.7, 55.7, 55.5, 25.4, 24.5, 0.4 ppm; IR (ATR): $\tilde{v}=3060,2954,2059,1619$, 1596, 1449, 1422, 1236, 1113, 1020, 909, $836 \mathrm{~cm}^{-1}$; HRMS (ESI): calcd. for $\left[\mathrm{C}_{32} \mathrm{H}_{31} \mathrm{Co}_{1} \mathrm{O}_{2} \mathrm{Si}\right]^{+}$, [MCO-I'] $]^{+}$661.0475; found: 661.0493; [a] $]_{D}^{20}:-109.9\left(c=0.30, \mathrm{CHCl}_{3}\right)$.

\section{Cobalt complex Co6}

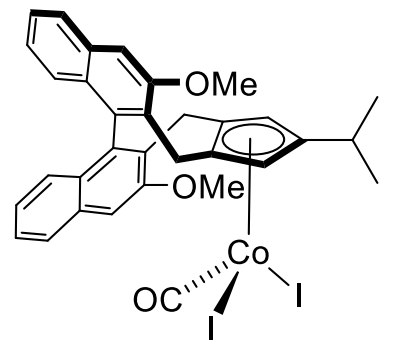

In an $\mathrm{N}_{2}$-filled glovebox, a flame-dried Schlenk tube was charged with $\mathrm{Co}_{2}(\mathrm{CO})_{8}(90.0 \mathrm{mg}, 264 \mu \mathrm{mol}, 0.55$ equiv.). A solution of $\mathbf{L 6}(214 \mathrm{mg}$, $479 \mu \mathrm{mol}, 1.00$ equiv.) in dry DCM $(10.0 \mathrm{~mL})$ was added. The Schlenk tube was connected to a Schlenk line for $\mathrm{CO}$ evolution and stirred at $37^{\circ} \mathrm{C}$ for $6 \mathrm{~h}$. The resulting solution was evaporated by Schlenk technique. A solution of iodine ( $66.9 \mathrm{mg}, 264 \mu \mathrm{mol}, 0.55$ equiv.) in dry degassed $\mathrm{Et}_{2} \mathrm{O}$ $(6.00 \mathrm{~mL})$ was added to the solid black residue. The reaction vessel was connected to a Schlenk line to release carbon monoxide and the dark violet mixture was stirred at room temperature for 1 h. All volatiles were evaporated and product was purified by silica gel column chromatography (gradient: $\mathrm{DCM} /$ pentane $=0 \% \rightarrow 50 \%$ ). The dark violet fractions were collected and concentrated under reduced pressure to obtain $155 \mathrm{mg}$ ( $197 \mu \mathrm{mol}, 41 \%$ yield) of the desired product as a black solid. ${ }^{1} \mathrm{H}$ NMR $\left(600 \mathrm{MHz}, \mathrm{CDCl}_{3}\right) \delta=7.88(\mathrm{~d}, J=8.3 \mathrm{~Hz}, 1 \mathrm{H}), 7.80(\mathrm{~d}, J=8.2 \mathrm{~Hz}, 1 \mathrm{H}), 7.47-7.39$ $(\mathrm{m}, 3 \mathrm{H}), 7.23(\mathrm{~s}, 1 \mathrm{H}), 7.15-7.07(\mathrm{~m}, 3 \mathrm{H}), 7.00(\mathrm{~d}, J=8.5 \mathrm{~Hz}, 1 \mathrm{H}), 5.57(\mathrm{~s}, 1 \mathrm{H}), 5.50(\mathrm{~s}, 1 \mathrm{H}), 4.11$ (s, 3H), $4.03(\mathrm{~d}, J=16.3 \mathrm{~Hz}, 1 \mathrm{H}), 3.96(\mathrm{~s}, 3 \mathrm{H}), 3.78(\mathrm{~d}, J=13.9 \mathrm{~Hz}, 1 \mathrm{H}), 3.59$ (hept, $J=7.0 \mathrm{~Hz}$, $1 \mathrm{H}$ ), 3.41 (d, $J=13.9 \mathrm{~Hz}, 1 \mathrm{H}), 2.75(\mathrm{~d}, J=16.3 \mathrm{~Hz}, 1 \mathrm{H}), 1.41(\mathrm{~d}, J=7.0 \mathrm{~Hz}, 3 \mathrm{H}), 1.39$ (d, $J=7.0$ $\mathrm{Hz}, 3 \mathrm{H}) \mathrm{ppm} ;{ }^{13} \mathrm{C}\left\{{ }^{1} \mathrm{H}\right\}$ NMR $\left(150 \mathrm{MHz}, \mathrm{CDCl}_{3}\right) \delta=198.3,155.8,154.7,138.1,137.0,134.3,133.9$, 127.3, 127.23, 127.18, 127.1, 127.0, $126.9(2 \times C)$, 126.8, 126.7, 124.6, 124.5, 124.3, 110.4, 106.33, 106.27, 100.1, 98.3, 95.7, 93.9, 55.7, 55.5, 27.6, 25.7, 24.4, 24.0, 22.5 ppm; IR (ATR): $\widetilde{v}=3062,3003,2961,2054,1596,1449,1423,1329,1296,1239,1166,1113,1019,906 \mathrm{~cm}^{-1}$; HRMS (APPI): calcd. for $\left[\mathrm{C}_{32} \mathrm{H}_{29} \mathrm{ColO}_{2}\right]^{+}, \quad$ [M-CO-l $]^{+}$: 631.0538; found: 631.0528; $[\alpha]_{D}^{20}:-155.3\left(c=0.20, \mathrm{CHCl}_{3}\right)$. 


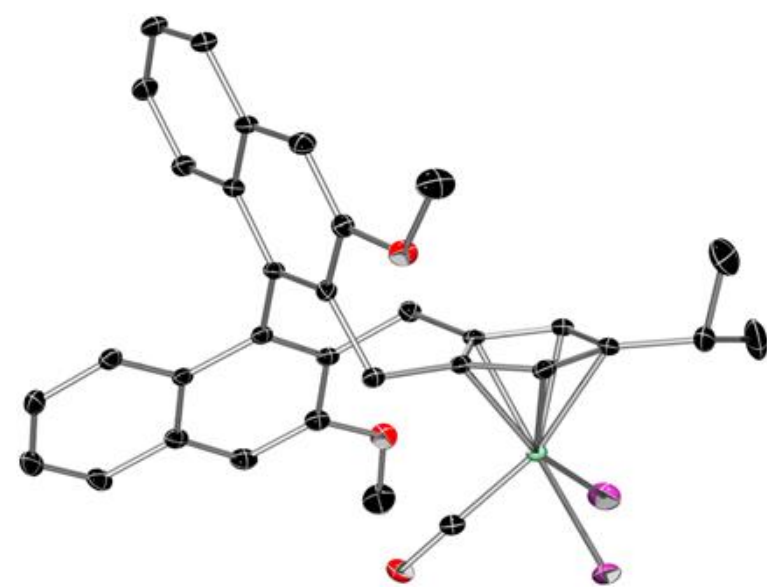

Figure 5: X-Ray Structure of Co6 (CCDC: 1898844).

\section{Cobalt complex Co7}

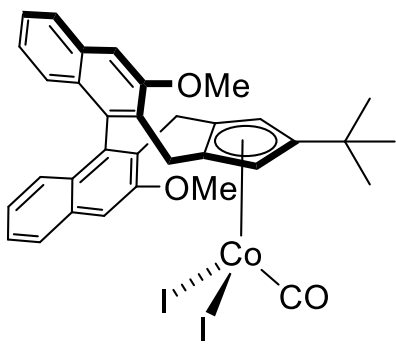

In a $\mathrm{N}_{2}$ filled glovebox, a flame dried Schlenk tube was charged with $\mathrm{Co}_{2}(\mathrm{CO})_{8}$ (129 mg, $358 \mu \mathrm{mol}, 1.10$ equiv.). A solution of $\mathbf{L 6}$ (150 mg, $326 \mu \mathrm{mol}, 1.00$ equiv. $)$ in dry DCM $(5.00 \mathrm{~mL})$ was added. CO gas was bubbled through the resulting solution for $5 \mathrm{~min}$ at $20^{\circ} \mathrm{C}$. The black reaction mixture was stirred at $37^{\circ} \mathrm{C}$ for $6 \mathrm{~h}$ under $\mathrm{CO}$ atmosphere. All volatiles were evaporated by Schlenk technique. A solution of iodine (99.0 mg, $391 \mu \mathrm{mol}, 1.20$ equiv.) in dry degassed $\mathrm{Et}_{2} \mathrm{O}(5.00 \mathrm{~mL})$ was added solution to the black residue. The reaction vessel was connected to a Schlenk line to release carbon monoxide and the dark violet mixture was stirred at room temperature for $1 \mathrm{~h}$. All volatiles were evaporated and the residue was purified by column chromatography on silica gel (gradient: DCM/pentane $=0 \% \rightarrow$ $50 \%)$. The dark violet fractions were collected and concentrated under reduced pressure to obtain $210 \mathrm{mg}\left(262 \mu \mathrm{mol}, 80 \%\right.$ yield) of the desired product as a black solid. ${ }^{1} \mathrm{H} \mathbf{~ N M R}\left(500 \mathrm{MHz}, \mathrm{CDCl}_{3}\right)$ $\delta=7.88(\mathrm{~d}, J=8.2 \mathrm{~Hz}, 1 \mathrm{H}), 7.80(\mathrm{~d}, J=8.1 \mathrm{~Hz}, 1 \mathrm{H}), 7.48-7.39(\mathrm{~m}, 2 \mathrm{H}), 7.39(\mathrm{~s}, 1 \mathrm{H}), 7.24(\mathrm{~s}$, $1 \mathrm{H}), 7.16-7.07(\mathrm{~m}, 3 \mathrm{H}), 7.01(\mathrm{~d}, J=8.4 \mathrm{~Hz}, 1 \mathrm{H}), 5.56(\mathrm{~s}, 1 \mathrm{H}), 5.45(\mathrm{~s}, 1 \mathrm{H}), 4.18(\mathrm{~d}, J=16.3 \mathrm{~Hz}$, $1 \mathrm{H}), 4.10(\mathrm{~s}, 3 \mathrm{H}), 3.96(\mathrm{~s}, 3 \mathrm{H}), 3.72(\mathrm{~d}, J=13.8 \mathrm{~Hz}, 1 \mathrm{H}), 3.49(\mathrm{~d}, J=13.8 \mathrm{~Hz}, 1 \mathrm{H}), 2.81(\mathrm{~d}, J=$ $16.3 \mathrm{~Hz}, 1 \mathrm{H}), 1.54(\mathrm{~s}, 9 \mathrm{H}) \mathrm{ppm} ;{ }^{13} \mathrm{C}\left\{{ }^{1} \mathrm{H}\right\} \mathbf{N M R}\left(151 \mathrm{MHz}, \mathrm{CDCl}_{3}\right), \delta=197.9,156.1,154.7,138.1$, 137.0, 134.4, 133.9, 127.4, 127.20, 127.17, 127.1, 127.02, 127.0, 126.9, 126.81, 126.78, 124.7, 124.6, 124.2, 114.0, $106.3(2 \times \mathrm{C}), 100.2,98.0,94.5,91.8,55.7,55.4,32.5,32.0,25.7,24.3 \mathrm{ppm}$; IR (ATR): $\tilde{v}=3063,2958,2925,2054,1618,1596,1449,1228,1113,1019,906,832,729 \mathrm{~cm}^{-}$

1; HRMS (ESI): calcd. for, $\left[\mathrm{C}_{33} \mathrm{H}_{31} \mathrm{ColO}_{2}\right]^{+}$, [M-CO-I $]^{+}$: 645.0706, found 645.0725; $[\alpha]_{D}^{20:}-104.4\left(c=0.30, \mathrm{CHCl}_{3}\right)$. 


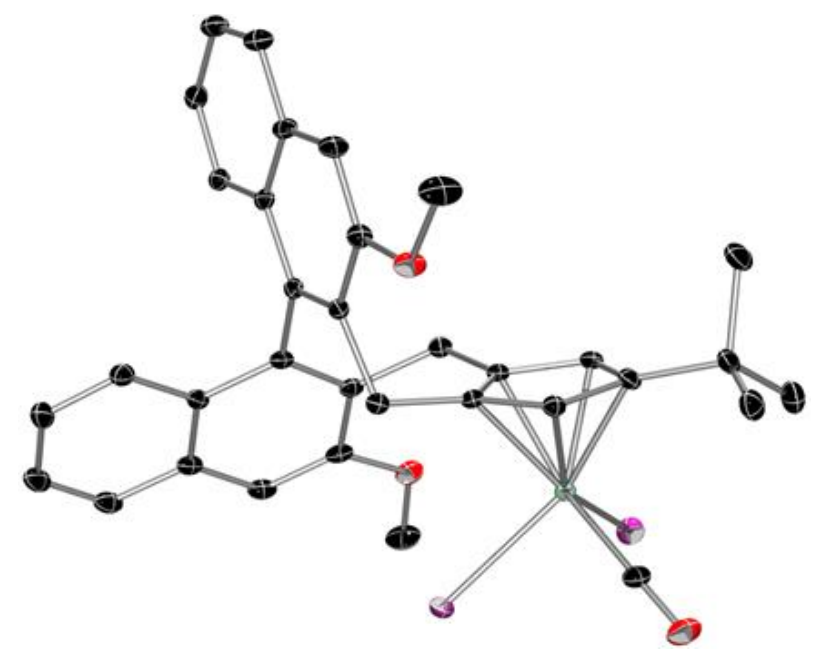

Figure 6: X-Ray Structure of Co7 (CCDC: 1898852).

Table 1: Optimisation table for complexation of L7:

\begin{tabular}{|l|l|l|l|l|}
\hline Entry & $\mathbf{C o}_{2}(\mathbf{C O})_{8}$ [equiv.] & Atmosphere & $\mathbf{I}_{2}$ [equiv.] & Yield [\%] \\
\hline 1 & 0.55 & $\mathrm{~N}_{2}$ & 0.55 & 40 \\
\hline 2 & 0.55 & $\mathrm{CO}$ & 0.55 & 55 \\
\hline 3 & 1.1 & $\mathrm{CO}$ & 1.2 & 80 \\
\hline
\end{tabular}

\section{$N$-Chloroamides}<smiles>O=C(NCl)c1ccccc1</smiles><smiles>COc1ccc(C(=O)NCl)cc1</smiles><smiles>Cc1ccc(C(=O)NCl)cc1</smiles><smiles>Cc1cccc(C(=O)NCl)c1</smiles><smiles>O=C(NCl)c1ccc(Br)cc1</smiles><smiles>O=C(NCl)c1ccc(Cl)cc1</smiles><smiles>O=C(NCl)c1ccc(F)cc1</smiles>

Figure 7: N-Chloroamides 1a-1g.

$\mathrm{N}$-Chloroamides $1 \mathrm{a}-\mathbf{1} \mathbf{g}$ were synthesized following the procedure of Zhu et al. ${ }^{7}$ 


\section{Styrenes, Acrylates and Other Vinyl Compounds}

All styrenes, acrylates and other vinyl compounds were obtained from commercial suppliers. Liquid compounds with stabilizers were purified prior to their use by column chromatography on aluminium oxide (basic, Brockman 1, 50-200 $\mu \mathrm{m}, 60 \AA$ A). Solid compounds were used as supplied. 


\section{Asymmetric Co(III) Catalyzed Reaction}

\section{General Procedure for Optimization Table}

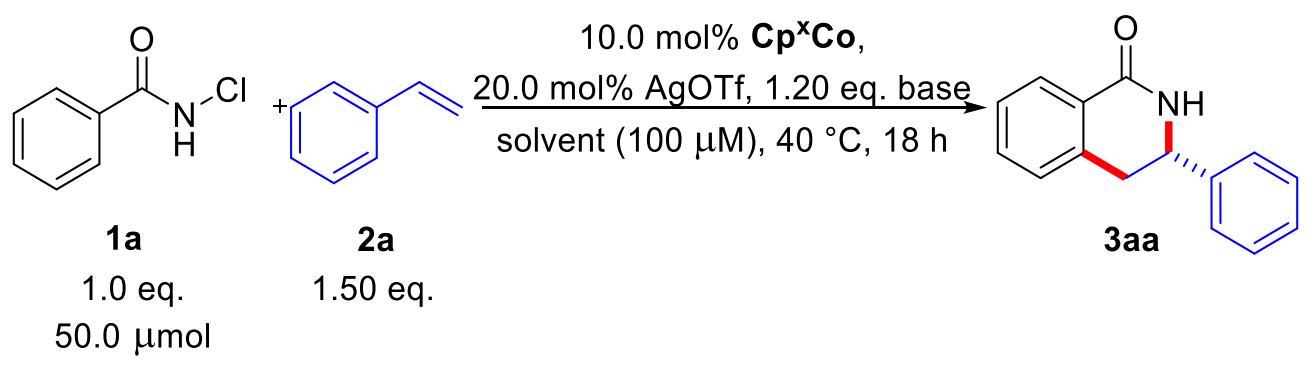

Scheme 2: Reaction procedure for optimizing the conditions.

Cobalt catalyst $(5.00 \mu \mathrm{mol}, 10.0 \mathrm{~mol} \%)$ was loaded into a flame dried vessel under air. Inside an $\mathrm{N}_{2}$-filled glovebox, the respective base $(60.0 \mu \mathrm{mol}, 1.20$ equiv. $)$, silver triflate $(2.57 \mathrm{mg}, 10.0 \mu \mathrm{mol}$, $20.0 \mathrm{~mol} \%)$ and the respective solvent $(250 \mu \mathrm{L})$ were added. The tube was sealed with a septum and the suspension was stirred at $20^{\circ} \mathrm{C}$ for $5 \mathrm{~min}$. Styrene (8.62 $\mu \mathrm{L}, 75.0 \mu \mathrm{mol}, 1.50$ equiv.) and a solution of $\mathrm{N}$-chlorobenzamide $(7.78 \mathrm{mg}, 50.0 \mu \mathrm{mol}, 1.00$ equiv.) in the respective solvent $(250 \mu \mathrm{L})$ were added and the resulting brown mixture was stirred for $18 \mathrm{~h}$ at $40^{\circ} \mathrm{C}$ in the absence of light. The reaction was then quenched by addition of $\mathrm{AcOH}(100 \mu \mathrm{L})$ and EtOAc $(500 \mu \mathrm{L})$, stirred for $15 \mathrm{~min}$ at $20^{\circ} \mathrm{C}$, and subsequently filtered through a pad of silica gel in vacuo. The resulting crude mixture was analyzed by quantitative $\mathrm{NMR}$ with $\mathrm{CH}_{2} \mathrm{Br}_{2}$ as the internal standard. For er determination, the crude mixture was purified by column chromatography on silica gel (eluent pentane/EtOAc 15\% $\rightarrow 25 \%$ ) and analyzed by HPLC using a chiral stationary phase (Chiralpak ID, 4.6 x $250 \mathrm{~mm} ; 20 \%$ i-PrOH/hexane, $1.0 \mathrm{~mL} / \mathrm{min}, 254 \mathrm{~nm})$.

\section{General Procedure A (5.00 mol\% Catalyst Loading)}

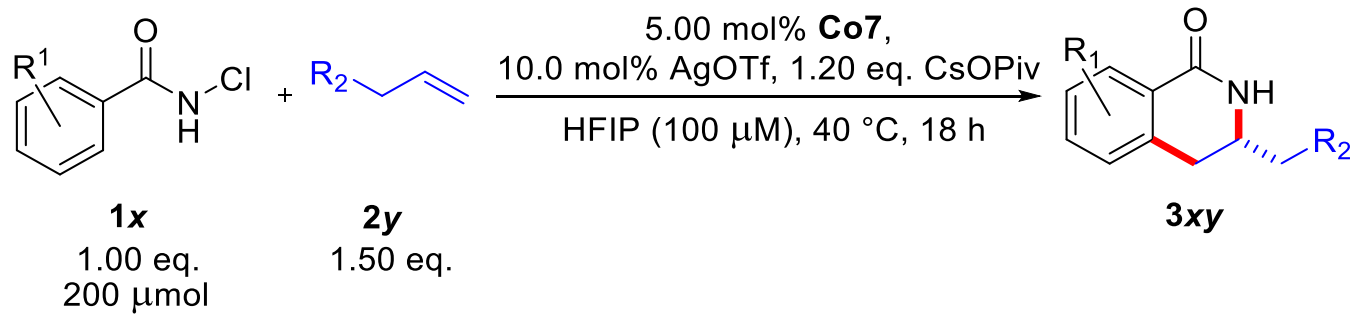

Scheme 3: Conditions for general procedure A. 
Cobalt catalyst Co7 (8.00 mg, $10.0 \mu \mathrm{mol}, 5.00$ mol\%) was loaded into a flame-dried reaction vessel. In $\mathrm{N}_{2}$-filled glovebox, caesium pivalate $(56.2 \mathrm{mg}, 240 \mu \mathrm{mol}, 1.20$ equiv.) and silver triflate (5.14 mg, $20.0 \mu \mathrm{mol}, 10.0 \mathrm{~mol} \%$ ) were added. The reaction vessel was sealed with a septum and dry, degassed HFIP $(1.00 \mathrm{~mL})$ was added. The resulting mixture was stirred at $20^{\circ} \mathrm{C}$ for $5 \mathrm{~min}$. Then, the respective vinyl (300 $\mu \mathrm{mol}, 1.50$ equiv.) and a solution of the respective $N$-chloroamide $(200 \mu \mathrm{mol}, 1.00$ equiv. $)$ in HFIP $(1.00 \mathrm{~mL})$ were added. The reaction mixture was stirred at $40{ }^{\circ} \mathrm{C}$ for $18 \mathrm{~h}$ in the absence of light. It was then quenched by addition of $\mathrm{AcOH}(0.200 \mathrm{~mL})$ and $\mathrm{EtOAc}$ $(1.00 \mathrm{~mL})$. The crude reaction mixture was filtered through a pad of silica gel, all volatiles were evaporated and the residue was purified by column chromatography on silica gel (pentane/EtOAc $15 \% \rightarrow 25 \%)$.

\section{General Procedure B (5.00 mol\% Catalyst Loading, Solid Reactant)}

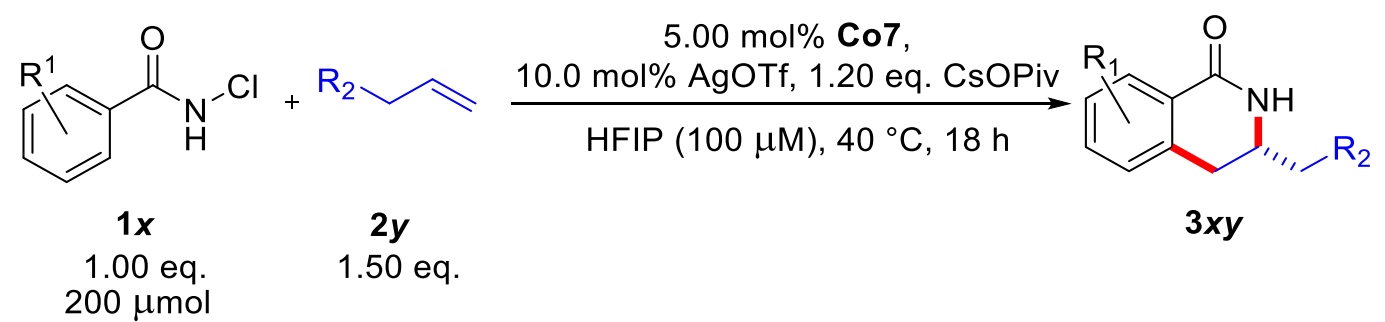

Scheme 4: Conditions for general procedure B.

Cobalt catalyst Co7 (8.00 mg, $10.0 \mu \mathrm{mol}, 5.00 \mathrm{~mol} \%)$ was loaded into a flame-dried reaction vessel. In an $\mathrm{N}_{2}$-filled glovebox, caesium pivalate (56.2 mg, $240 \mu \mathrm{mol}, 1.20$ equiv.) and silver triflate $(5.14 \mathrm{mg}, 20.0 \mu \mathrm{mol}, 10.0 \mathrm{~mol} \%)$ were added. The reaction vessel was sealed with a septum and dry, degassed HFIP $(1.00 \mathrm{~mL})$ was added. The resulting mixture was stirred at $20^{\circ} \mathrm{C}$ for $5 \mathrm{~min}$. Then, HFIP (1.00 mL), the respective vinyl $(300 \mu \mathrm{mol}, 1.50$ equiv.) and the respective solid $\mathrm{N}$-chloroamide (200 $\mathrm{mmol}, 1.00$ equiv.) were added. The reaction mixture was stirred at $40{ }^{\circ} \mathrm{C}$ for $18 \mathrm{~h}$ in the absence of light. It was then quenched by addition of $\mathrm{AcOH}(0.200 \mathrm{~mL})$ and $\mathrm{EtOAc}$ $(1.00 \mathrm{~mL})$. The crude reaction mixture was filtered through a pad of silica gel, all volatiles were evaporated and the residue was purified by column chromatography on silica gel (gradient: EtOAc/pentane $=15 \% \rightarrow 25 \%$ ). 


\section{General Procedure C (10.0 mol\% Catalyst Loading)}

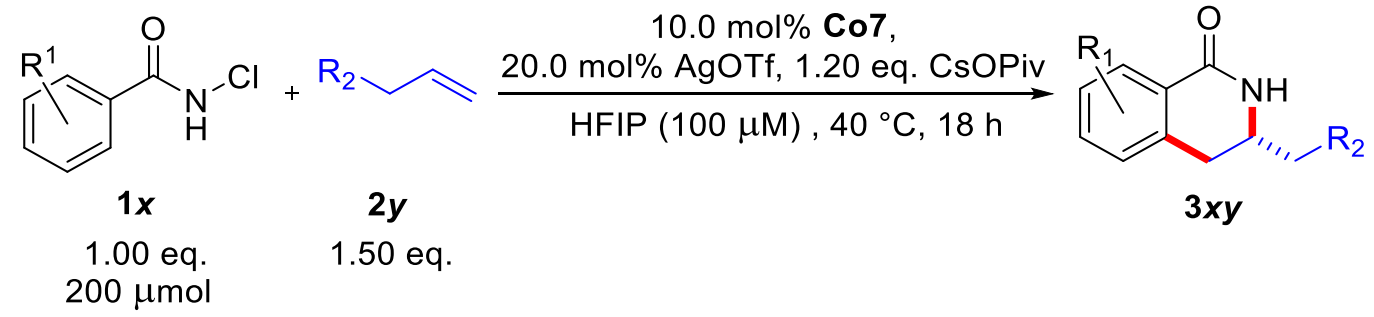

Scheme 5: Conditions for general procedure C.

Cobalt catalyst Co7 (16.0 mg, $20.0 \mu \mathrm{mol}, 10.0 \mathrm{~mol} \%)$ was loaded in a flame-dried reaction vessel. In an $\mathrm{N}_{2}$-filled glovebox, caesium pivalate ( $56.2 \mathrm{mg}, 240 \mu \mathrm{mol}, 1.20$ equiv.) and silver triflate (10.3 mg, $40.0 \mu \mathrm{mol}, 20.0 \mathrm{~mol} \%$ ) were added. The reaction vessel was sealed with a septum and dry, degassed HFIP $(1.00 \mathrm{~mL})$ was added. The resulting mixture was stirred at $20^{\circ} \mathrm{C}$ for $5 \mathrm{~min}$. Then, the respective vinyl ( $300 \mu \mathrm{mol}, 1.50$ equiv.) and a solution of the respective $\mathrm{N}$-chloroamide (200 $\mu \mathrm{mol}, 1.00$ equiv.) in HFIP $(1.00 \mathrm{~mL})$ were added. The reaction mixture was stirred at $40^{\circ} \mathrm{C}$ for $18 \mathrm{~h}$ in the absence of light. It was then quenched by addition of $\mathrm{AcOH}(0.200 \mathrm{~mL})$ and EtOAc $(1.00 \mathrm{~mL})$. The crude reaction mixture was filtered through a pad of silica gel, all volatiles were evaporated and the residue was purified by column chromatography on silica gel (pentane/EtOAc $=15 \% \rightarrow 25 \%)$.

\section{Racemic reaction}

The racemic samples for enantioenriched dihydroisoquinolinones were obtained by the procedure of Zhu et al. utilizing $\mathrm{Cp}^{*} \mathrm{Co}(\mathrm{CO}) \mathrm{I}_{2}{ }^{8}$

\section{(-)-(S)-3-phenyl-3,4-dihydroisoquinolin-1(2H)-one (3aa)}

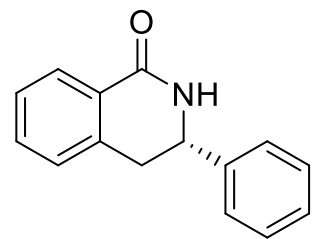

General Procedure A. Yield: 87\% (39.0 mg, $175 \mu \mathrm{mol})$, white solid. The spectra match with the literature values ${ }^{9} .{ }^{1} \mathrm{H}$ NMR $\left(400 \mathrm{MHz}, \mathrm{CDCl}_{3}\right) \delta=8.11$ (dd, $J=7.7,1.4 \mathrm{~Hz}, 1 \mathrm{H}), 7.46$ (td, $J=7.5,1.5 \mathrm{~Hz}, 1 \mathrm{H}), 7.43-7.33(\mathrm{~m}, 6 \mathrm{H})$, $7.20(\mathrm{~d}, J=7.5 \mathrm{~Hz}, 1 \mathrm{H}), 6.09(\mathrm{~s}, 1 \mathrm{H}), 4.89(\mathrm{dd}, J=11.0,4.8 \mathrm{~Hz}, 1 \mathrm{H}), 3.21$ (dd, $J=15.7,11.0 \mathrm{~Hz}, 1 \mathrm{H}), 3.13(\mathrm{dd}, J=15.7,4.8 \mathrm{~Hz}, 1 \mathrm{H}) \mathrm{ppm}$; $\boldsymbol{R}_{\mathrm{f}}: 0.43($ hexane/EtOAc = 1/1); $[a]_{D}^{20}-161.0\left(c=1.00, \mathrm{CHCl}_{3}\right)$; Chiral HPLC: Chiralpak ID, $4.6 \times 250 \mathrm{~mm} ; 20 \% \mathrm{i}-\mathrm{PrOH} / \mathrm{hexane}$, $1.0 \mathrm{~mL} / \mathrm{min}, 254 \mathrm{~nm}$; $\operatorname{tr}$ (minor) = $18.6 \mathrm{~min}$, $\operatorname{tr}$ (major) $=20.1 \mathrm{~min}, 99.6 / 0.4 \mathrm{er}$. 


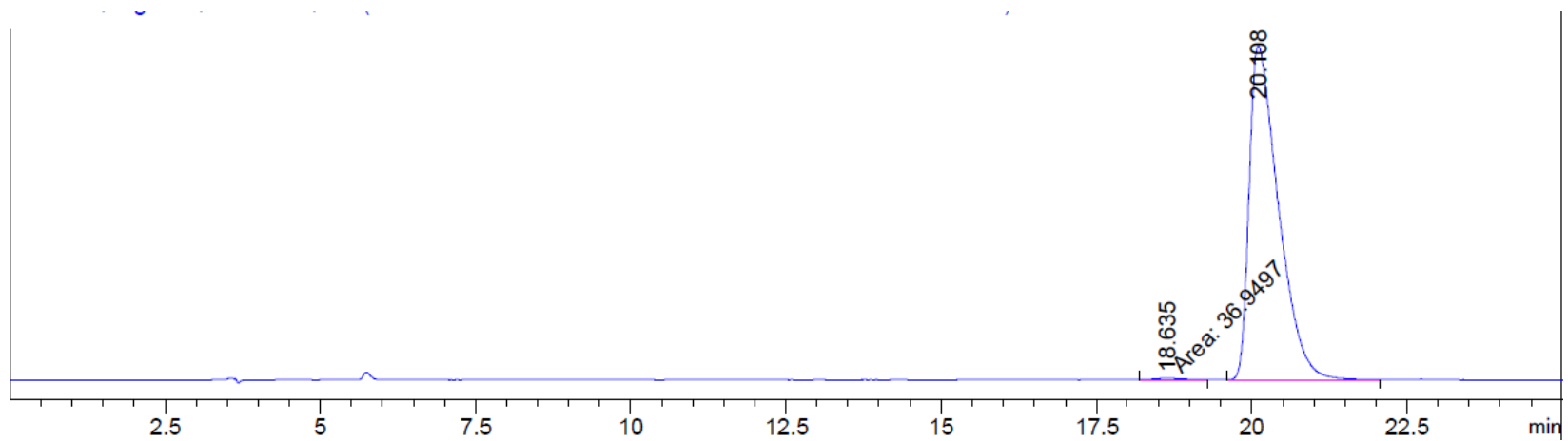

\begin{tabular}{|c|c|c|c|c|c|c|}
\hline $\begin{array}{c}e a k \\
\#\end{array}$ & $\begin{array}{c}\text { RetTime } \\
\text { [min] }\end{array}$ & Type & $\begin{array}{l}\text { Width } \\
\text { [min] }\end{array}$ & $\begin{array}{c}\text { Area } \\
{\left[\mathrm{mAU}^{*} \mathrm{~S}\right]}\end{array}$ & $\begin{array}{l}\text { Height } \\
\text { [mAU] }\end{array}$ & $\begin{array}{c}\text { Area } \\
\frac{8}{8}\end{array}$ \\
\hline & & & & ------- & & \\
\hline & & & & 36.94972 & 1.25841 & 405 \\
\hline 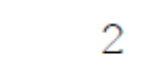 & & DD & 0.4946 & 9069.29102 & 276.54721 & 594 \\
\hline
\end{tabular}

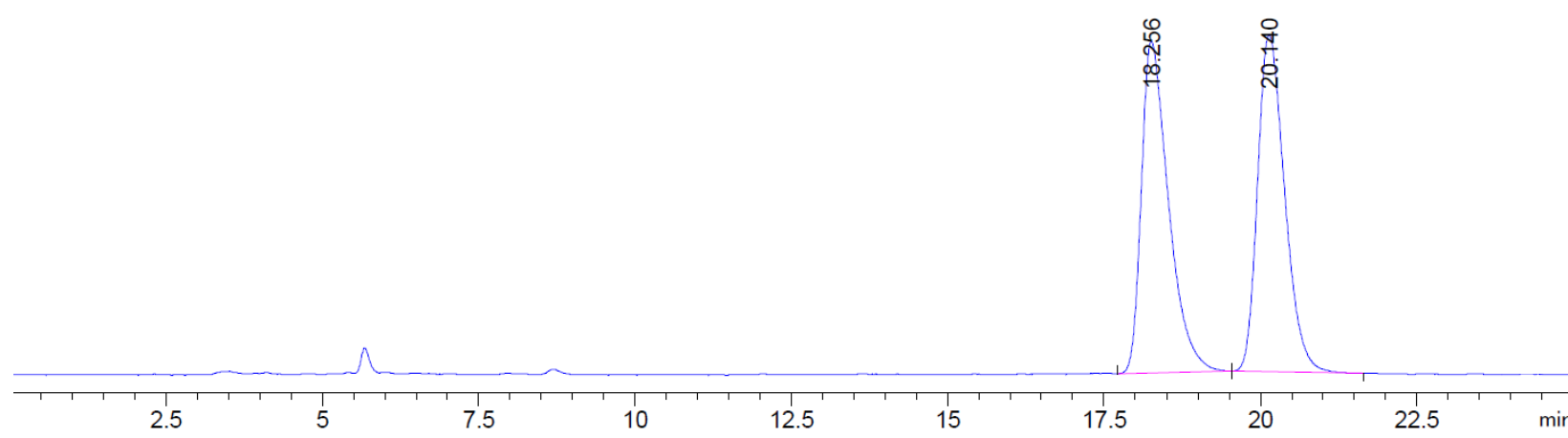

Peak RetTime Type Width Area Height Area

\begin{tabular}{|c|c|c|c|c|c|}
\hline \# & [min] & [min] & {$\left[\mathrm{mAU}{ }^{*} \mathrm{~s}\right]$} & [mAU] & $\%$ \\
\hline & & & & & \\
\hline 2 & $20.140 \mathrm{BB}$ & 0.4631 & 1499.77356 & 49.83626 & 50 \\
\hline
\end{tabular}

(-)-(S)-6-Methoxy-3-phenyl-3,4-dihydroisoquinolin-1(2H)-one (3ba)<smiles>COc1ccc2c(c1)CC(c1ccccc1)NC2=O</smiles>

General Procedure B. $10 \mathrm{~mol} \% \mathrm{Co} 7$ and $20 \mathrm{~mol} \%$ AgOTf loadings were used. Yield: $61 \%$ (31.2 mg, $123 \mu \mathrm{mol})$, brown solid. The spectra match with the literature values ${ }^{9} .{ }^{1} \mathrm{H}$ NMR $\left(400 \mathrm{MHz}, \mathrm{CDCl}_{3}\right) \delta=8.06(\mathrm{~d}, J=8.6$, $1 \mathrm{H}), 7.41-7.29(\mathrm{~m}, 5 \mathrm{H}), 6.87(\mathrm{dd}, J=8.6,2.2 \mathrm{~Hz}, 1 \mathrm{H}), 6.66(\mathrm{~d}, J=1.8$ Hz, 1H), 6.15 (s, 1H), 4.83 (dd, $J=10.5,5.2 \mathrm{~Hz}, 1 \mathrm{H}$ ), 3.83 (s, 3H), 3.16 (dd, $J=15.7,10.5 \mathrm{~Hz}$, $1 \mathrm{H}), 3.08(\mathrm{dd}, J=15.7,5.2 \mathrm{~Hz}, 1 \mathrm{H}) \mathrm{ppm} ; \boldsymbol{R}_{\mathrm{f}}: 0.40\left(\mathrm{CHCl}_{3} / \mathrm{MeOH} 40: 1\right) ;[\alpha]_{\mathrm{D}}^{20}:-152(c=1.00$, 
$\mathrm{CHCl}_{3}$ ); Chiral HPLC: Chiralpak IA, 4.6 x $250 \mathrm{~mm}$; 30\% i-PrOH/hexane, $1.0 \mathrm{~mL} / \mathrm{min}, 254 \mathrm{~nm}$; tr (minor) $=12.9 \mathrm{~min}, \operatorname{tr}($ major $)=16.4 \mathrm{~min}, 98.9 / 1.1 \mathrm{er}$.

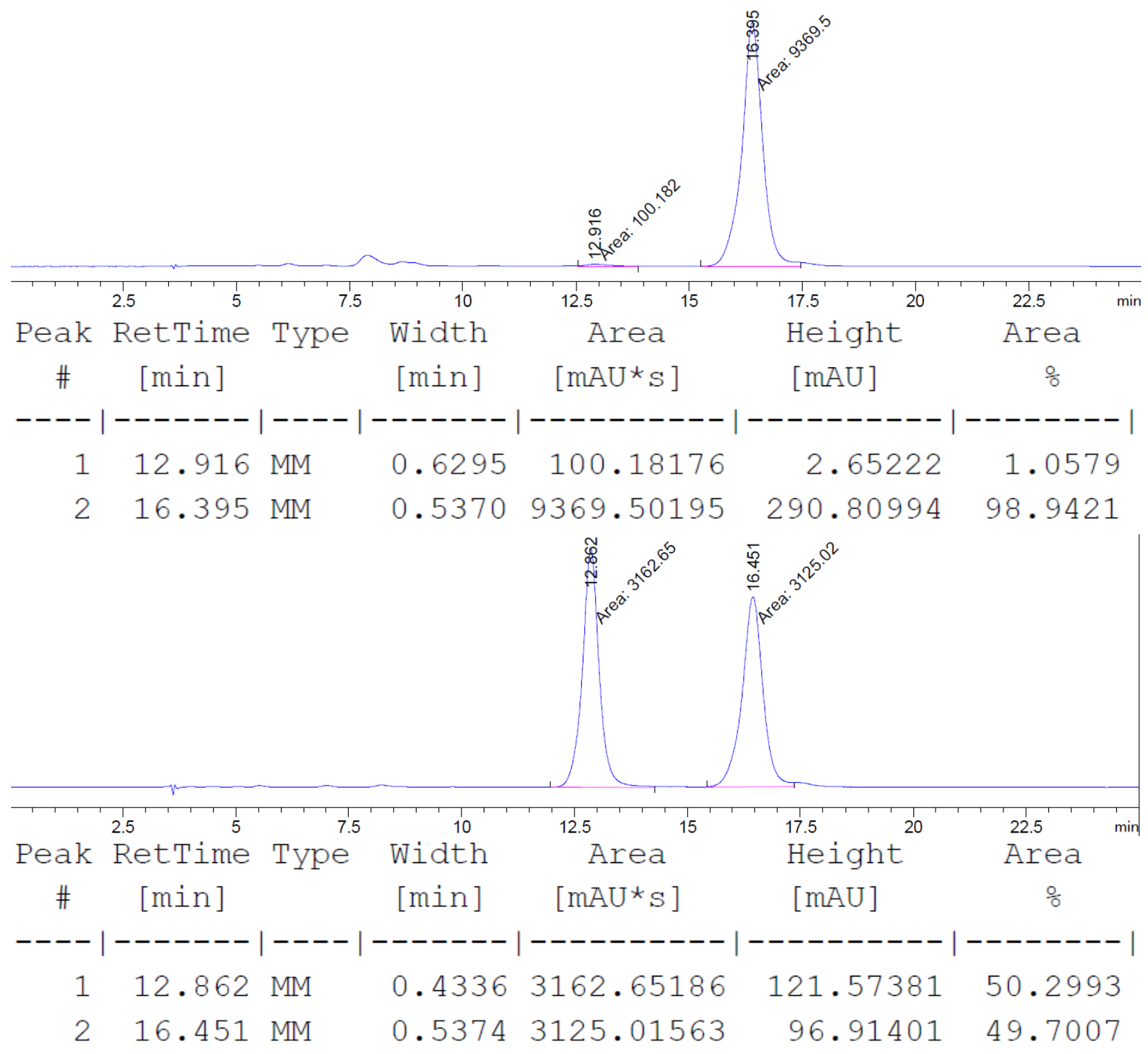

(-)-(S)-6-Methyl-3-phenyl-3,4-dihydroisoquinolin-1(2H)-one (3ca)<smiles>Cc1ccc2c(c1)C[C@@H](c1ccccc1)NC2=O</smiles>

General Procedure A. Yield: 63\% (30.0 mg, $126 \mu \mathrm{mol})$, white solid. The spectra match with the literature values ${ }^{9} .{ }^{1} \mathbf{H}$ NMR $\left(400 \mathrm{MHz}, \mathrm{CDCl}_{3}\right) \delta=$ $8.00(\mathrm{~d}, J=7.9 \mathrm{~Hz}, 1 \mathrm{H}), 7.42-7.30(\mathrm{~m}, 5 \mathrm{H}), 7.18(\mathrm{~d}, J=7.9 \mathrm{~Hz}, 1 \mathrm{H}), 6.99$ (s, 1H), $6.01(\mathrm{~s}, 1 \mathrm{H}), 4.84(\mathrm{dd}, J=10.8,4.8 \mathrm{~Hz}, 1 \mathrm{H}), 3.16$ (dd, $J=15.6$, $10.8 \mathrm{~Hz}, 1 \mathrm{H}$ ), 3.08 (dd, $J=15.6,4.8 \mathrm{~Hz}, 1 \mathrm{H}$ ), 2.38 (s, 3H) ppm; $\boldsymbol{R}_{\mathrm{f}}: 0.30$ (hexane/EtOAc = 2/1); $[\alpha]_{\mathrm{D}}^{20}:-163.2\left(c=1.00, \mathrm{CHCl}_{3}\right)$; Chiral HPLC: Chiralpak ID, 4.6 x $250 \mathrm{~mm} ; 20 \%$ i-PrOH/hexane, $1.0 \mathrm{~mL} / \mathrm{min}, 254 \mathrm{~nm}$; tr (minor) = $24.1 \mathrm{~min}$, tr (major) = $28.4 \mathrm{~min}, 98.7 / 1.3 \mathrm{er}$. 


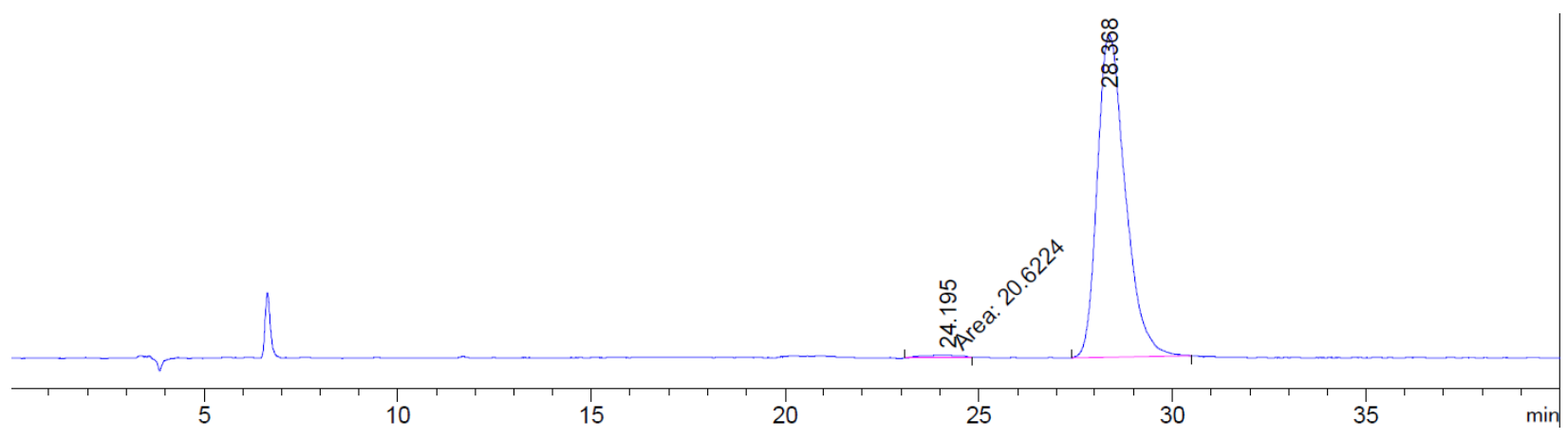

Peak RetTime Type Width Area Height Area
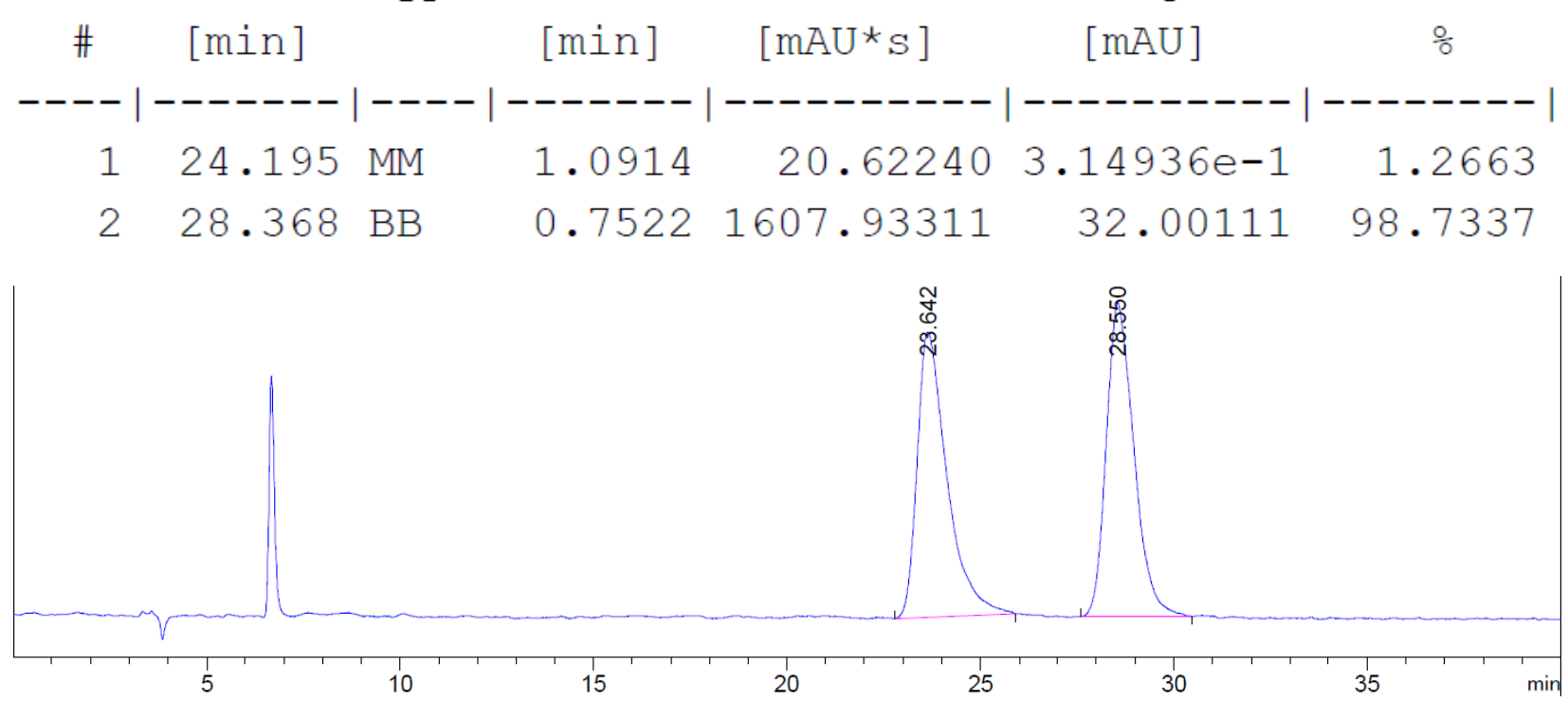

Peak RetTime Type Width Area Height Area

\begin{tabular}{|c|c|c|c|c|c|c|}
\hline \# & [min] & & [min] & {$\left[\mathrm{mAU}^{*} \mathrm{~s}\right]$} & [mAU] & $\frac{\circ}{0}$ \\
\hline & & & & & & \\
\hline 1 & 23.642 & BB & 0.7183 & 743.46063 & 13.84928 & 041 \\
\hline 2 & 28.550 & $\mathrm{BB}$ & 0.6928 & 758.35571 & 15.23226 & 50 \\
\hline
\end{tabular}

(-)-(S)-7-Methyl-3-phenyl-3,4-dihydroisoquinolin-1(2H)-one (3da)

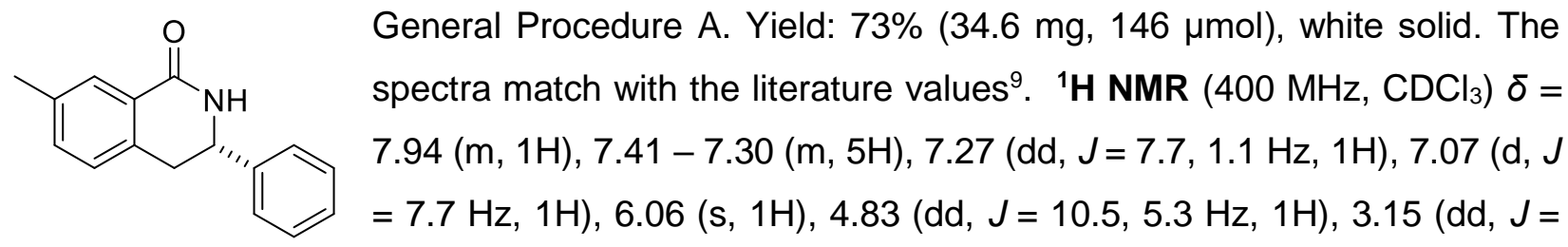

15.7, $10.5 \mathrm{~Hz}, 1 \mathrm{H}$ ), 3.10 (dd, $J=15.7,5.3 \mathrm{~Hz}, 1 \mathrm{H}$ ), 2.39 (s, 3H) ppm; $\boldsymbol{R}_{\mathrm{f}}: 0.30$ (hexane/EtOAc = 2/1); $[\alpha]_{\mathrm{D}}^{20}:-175.3\left(c=1.00, \mathrm{CHCl}_{3}\right)$; Chiral HPLC: Chiralpak IA, $4.6 \times 250 \mathrm{~mm} ; 10 \% i-$ $\mathrm{PrOH} /$ hexane, $1.0 \mathrm{~mL} / \mathrm{min}, 254 \mathrm{~nm}$; $\operatorname{tr}($ major $)=11.9 \mathrm{~min}$, tr $($ minor $)=13.7 \mathrm{~min}, 99.6 / 0.4 \mathrm{er}$. 


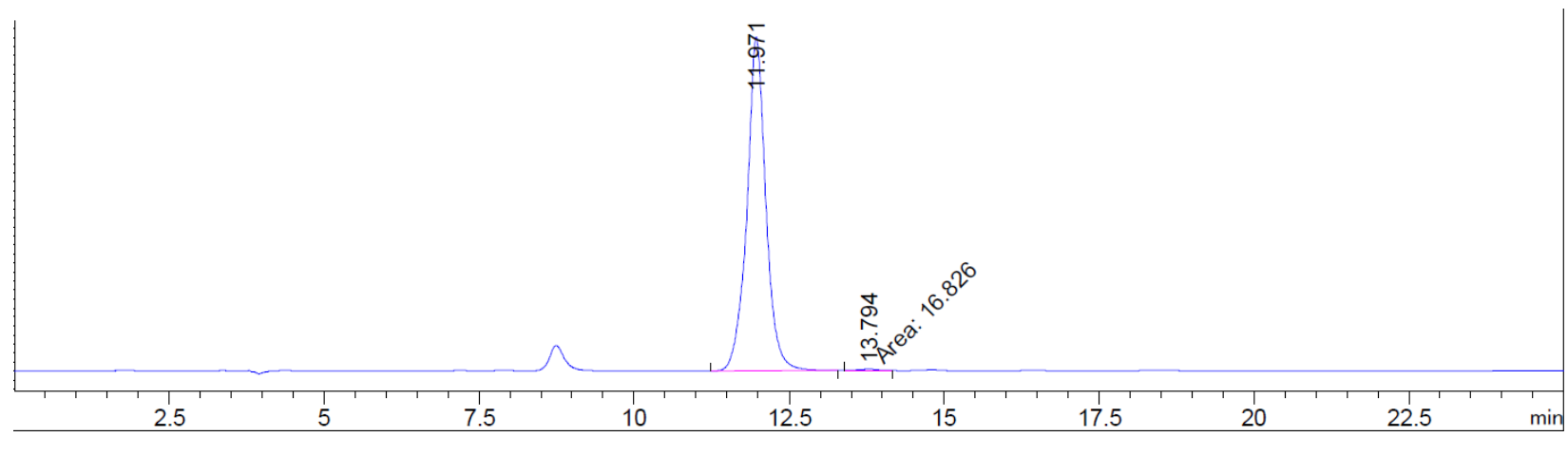

Peak RetTime Type Width Area Height Area

\begin{tabular}{|c|c|c|c|c|c|}
\hline \# & {$[\mathrm{min}]$} & {$[\mathrm{min}]$} & {$\left[\mathrm{mAU}{ }^{*} \mathrm{~S}\right]$} & {$[\mathrm{mAU}]$} & 응 \\
\hline & & & | ---------- & | ---- & --- \\
\hline 1 & $71 \mathrm{BB}$ & 0 . & 3959.29175 & 185.24413 & 99 \\
\hline 2 & $13.794 \mathrm{MM}$ & 3470 & 16.82598 & $8.08053 e-1$ & 0.4232 \\
\hline
\end{tabular}

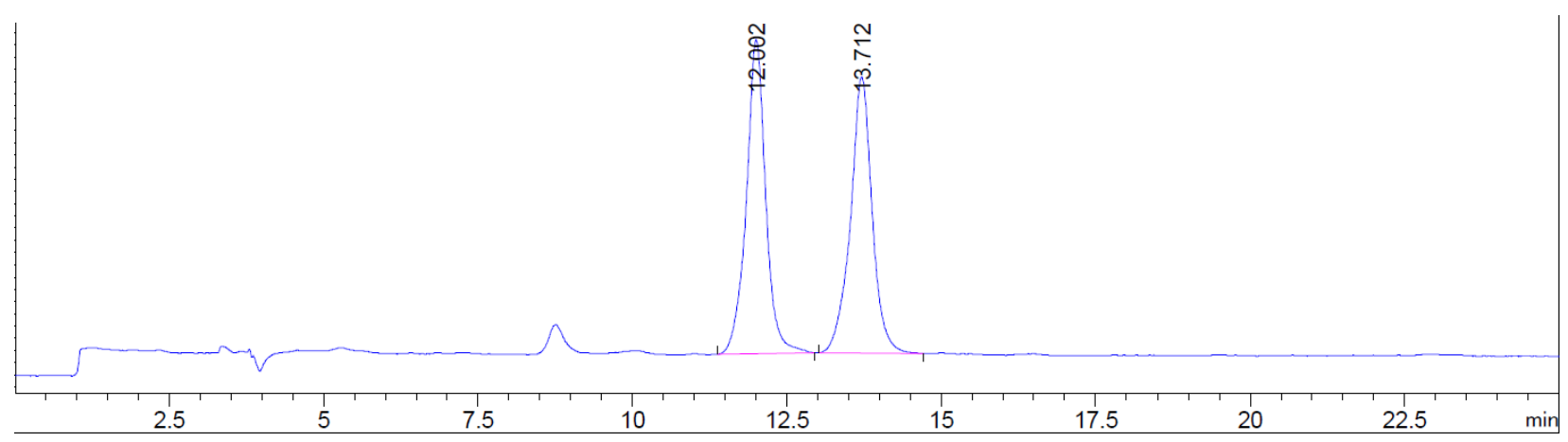

\begin{tabular}{|c|c|c|c|c|c|c|}
\hline $\begin{array}{c}\text { Peak } \\
\text { \# }\end{array}$ & $\begin{array}{c}\text { RetTime } \\
\text { [min] }\end{array}$ & Type & $\begin{array}{l}\text { Width } \\
{[\mathrm{min}]}\end{array}$ & $\begin{array}{c}\text { Area } \\
{\left[\mathrm{mAU}^{*} \mathrm{~s}\right]}\end{array}$ & $\begin{array}{l}\text { Height } \\
{[\mathrm{mAU}]}\end{array}$ & $\begin{array}{c}\text { Area } \\
\frac{\circ}{0}\end{array}$ \\
\hline & & & & & & \\
\hline 1 & 12.0 & & & 563 & 541 & 99 \\
\hline 2 & 13.7 & & 97 & 96527 & 22.61925 & 5701 \\
\hline
\end{tabular}

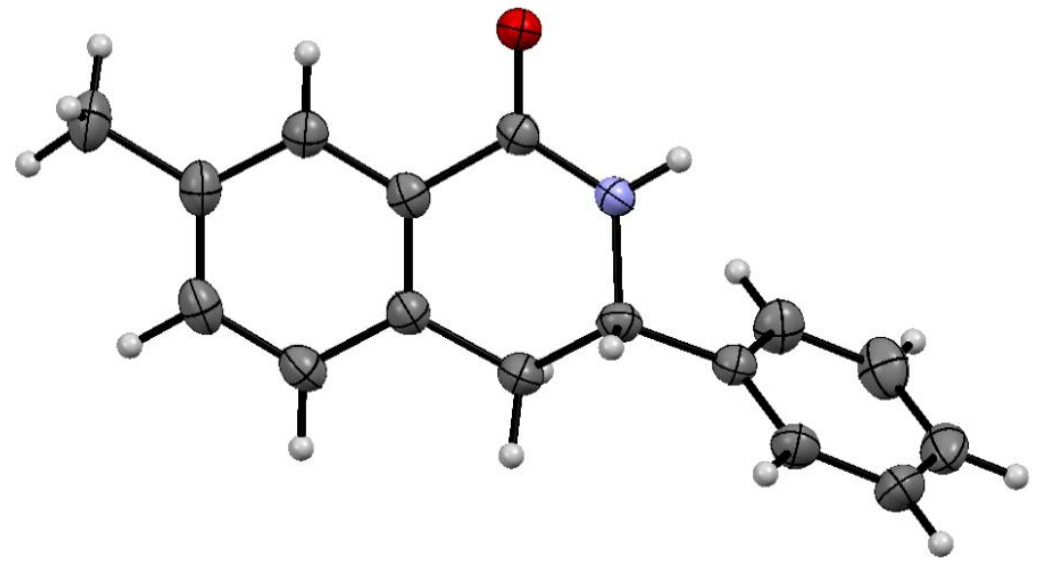

X-Ray structure of 3da (CCDC No: 1898858). 
(-)-(S)-6-Bromo-3-phenyl-3,4-dihydroisoquinolin-1(2H)-one (3ea)<smiles>O=C1N[C@@H](c2ccccc2)Cc2cc(Br)ccc21</smiles>

General Procedure B. Yield: 89\% (54.0 mg, $179 \mu \mathrm{mol})$, white solid. The spectra match with the literature values ${ }^{9} .{ }^{1} \mathbf{H}$ NMR $\left(400 \mathrm{MHz}, \mathrm{CDCl}_{3}\right) \delta=$ $7.93(\mathrm{~d}, J=8.2 \mathrm{~Hz}, 1 \mathrm{H}), 7.51$ (dd, $J=8.2,1.4 \mathrm{~Hz}, 1 \mathrm{H}), 7.44-7.33(\mathrm{~m}$, $6 \mathrm{H}), 6.11(\mathrm{~s}, 1 \mathrm{H}), 4.92(\mathrm{dd}, J=10.7,4.8 \mathrm{~Hz}, 1 \mathrm{H}), 3.19(\mathrm{dd}, J=15.9,10.7$ $\mathrm{Hz}, 1 \mathrm{H}), 3.11(\mathrm{dd}, J=15.9,4.8 \mathrm{~Hz}, 1 \mathrm{H}) \mathrm{ppm} ; \boldsymbol{R}_{\mathrm{f}}: 0.30($ hexane/EtOAc $=2 / 1) ;[\alpha]_{\mathrm{D}}^{20}:-113.5$ $\left(c=1.00, \mathrm{CHCl}_{3}\right)$; Chiral HPLC: Chiralpak ID, $4.6 \times 250 \mathrm{~mm} ; 30 \% i-\mathrm{PrOH} /$ hexane, $1.0 \mathrm{~mL} / \mathrm{min}$, $254 \mathrm{~nm}$; tr $($ minor $)=14.9 \mathrm{~min}, \operatorname{tr}($ major $)=17.7 \mathrm{~min}, 98.7 / 1.3 \mathrm{er}$.
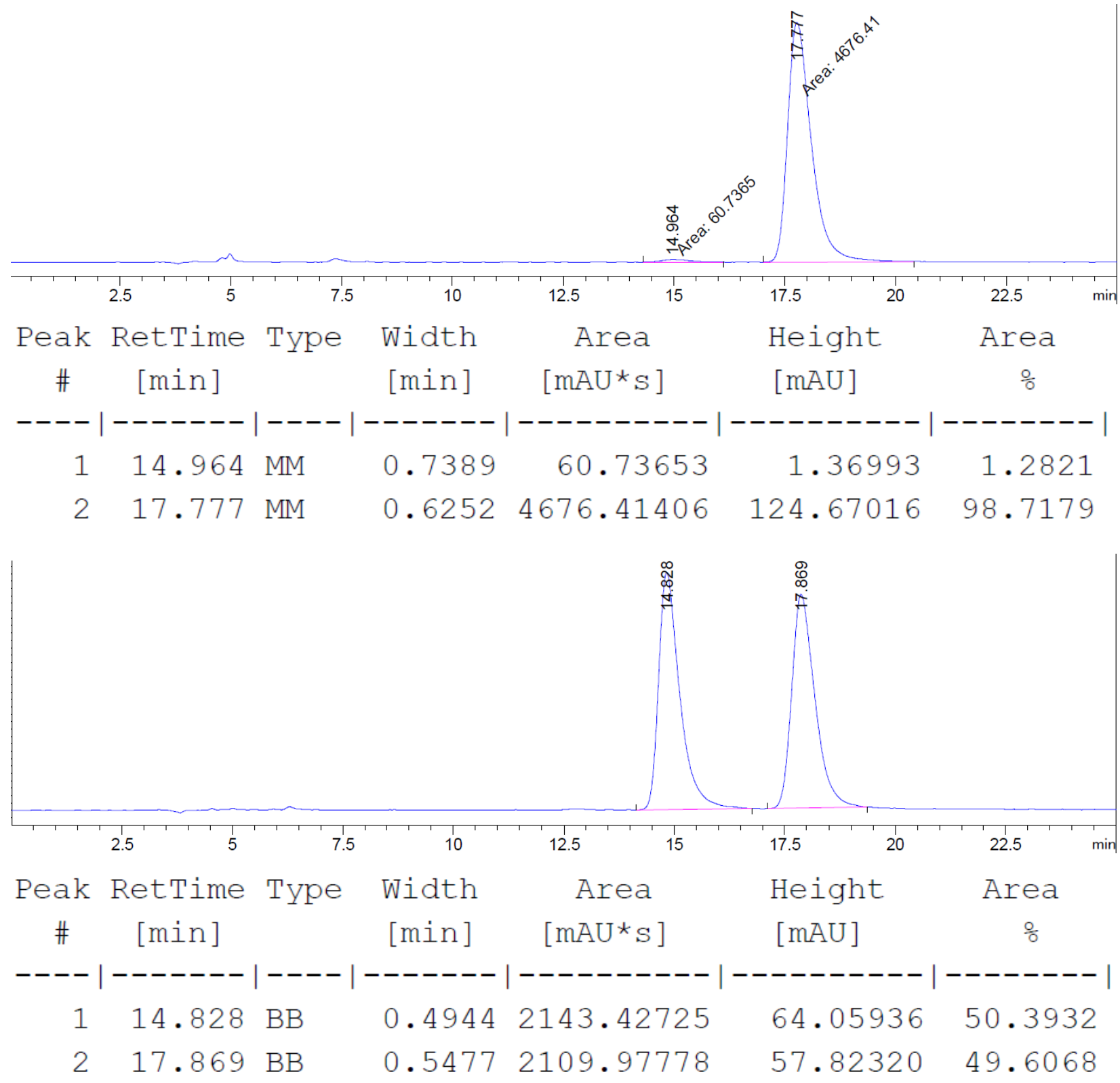


\section{(-)-(S)-6-Chloro-3-phenyl-3,4-dihydroisoquinolin-1(2H)-one (3fa)}<smiles>O=C1N[C@H](c2ccccc2)Cc2cc(Cl)ccc21</smiles>

General Procedure B. The spectra match with the literature values ${ }^{9}$. Yield: $82 \%(42.1 \mathrm{mg}, 163 \mu \mathrm{mol})$, white solid. ${ }^{1} \mathrm{H}$ NMR $\left(400 \mathrm{MHz}, \mathrm{CDCl}_{3}\right) \delta=$ $8.02(\mathrm{~d}, J=8.3 \mathrm{~Hz}, 1 \mathrm{H}), 7.42-7.28(\mathrm{~m}, 6 \mathrm{H}), 7.16(\mathrm{~s}, 1 \mathrm{H}), 6.47$ (br.s, $1 \mathrm{H})$, 4.84 (dd, $J=9.6,5.7 \mathrm{~Hz}, 1 \mathrm{H}$ ), 3.16 (dd, $J=16.9,9.6 \mathrm{~Hz}, 1 \mathrm{H}), 3.11$ (dd, $J$ $=16.9,5.7 \mathrm{~Hz}, 1 \mathrm{H})$ ppm; $\boldsymbol{R}_{\mathrm{f}}: 0.30$ (hexane/EtOAc = 2/1); [a] $]_{\mathrm{D}}^{20}:-126.8\left(c=1.00, \mathrm{CHCl}_{3}\right)$; Chiral HPLC: Chiralpak ID, 4.6 x 250 mm; 20\% i-PrOH/hexane, $1.0 \mathrm{~mL} / \mathrm{min}, 254 \mathrm{~nm}$; tr (minor) = 16.9 min, $\operatorname{tr}$ (major) $=20.1 \mathrm{~min}, 99.4 / 0.6 \mathrm{er}$.

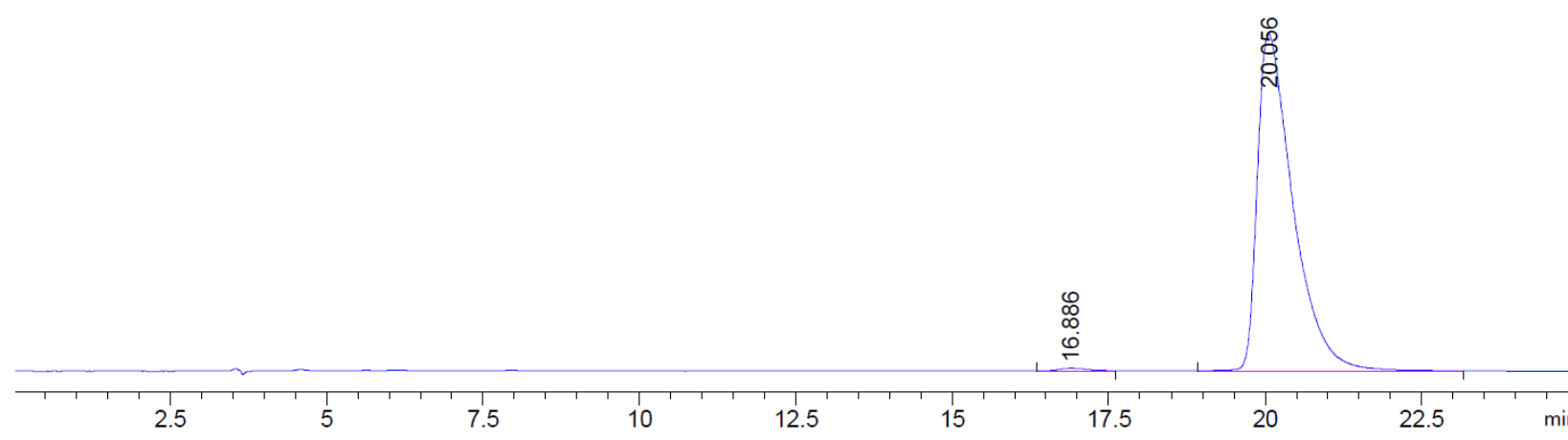

Peak RetTime Type Width Area Height Area
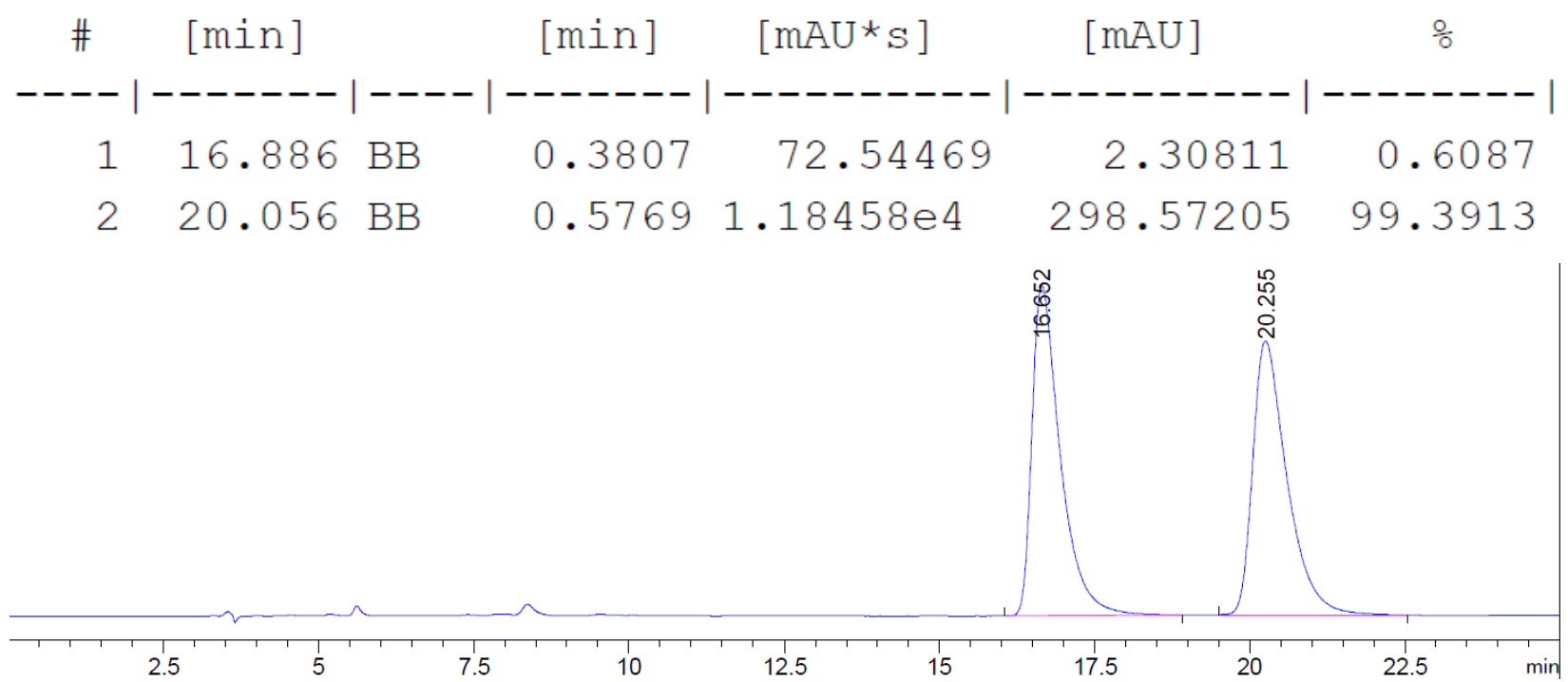

Peak RetTime Type Width Area Height Area

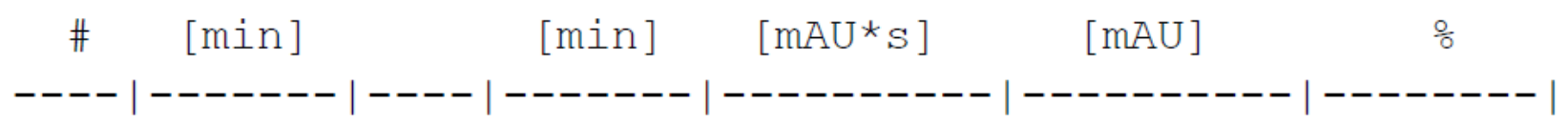

$1 \quad 16.652 \mathrm{BB} \quad 0.4760 \quad 4564.64844 \quad 143.13995 \quad 50.0703$

$\begin{array}{lllllll}2 & 20.255 & \mathrm{BB} & 0.5674 & 4551.83447 & 118.69341 & 49.9297\end{array}$ 


\section{(-)-(S)-6-Fluoro-3-phenyl-3,4-dihydroisoquinolin-1(2H)-one (3ga)}<smiles>O=C1N[C@H](c2ccccc2)Cc2cc(F)ccc21</smiles>

General Procedure B. Yield: 87\% (41.9 mg, $174 \mu \mathrm{mol})$, white solid. The spectra match with the literature values ${ }^{9} .{ }^{1} \mathbf{H}$ NMR $\left(400 \mathrm{MHz}, \mathrm{CDCl}_{3}\right) \delta=$ $8.14(\mathrm{dd}, J=8.6,5.8 \mathrm{~Hz}, 1 \mathrm{H}), 7.43-7.33(\mathrm{~m}, 5 \mathrm{H}), 7.06(\mathrm{dt}, J=8.6,2.3$ $\mathrm{Hz}, 1 \mathrm{H}), 6.90$ (dd, $J=8.7,2.1 \mathrm{~Hz}, 1 \mathrm{H}), 5.94$ (s, 1H), 4.87 (dd, $J=11.0,4.8$ $\mathrm{Hz}, 1 \mathrm{H}), 3.21(\mathrm{dd}, J=15.9,11.0 \mathrm{~Hz}, 1 \mathrm{H}), 3.12(\mathrm{dd}, J=15.9,4.8 \mathrm{~Hz}, 1 \mathrm{H}) \mathrm{ppm} ;{ }^{19} \mathrm{~F}$ NMR $(376 \mathrm{MHz}$, $\left.\mathrm{CDCl}_{3}\right) \delta=-106.5(\mathrm{td}, J=8.7,5.8 \mathrm{~Hz}) \mathrm{ppm} ; \boldsymbol{R}_{\mathrm{f}}: 0.30$ (hexane/EtOAc $\left.=2 / 1\right) ;[\boldsymbol{\alpha}]_{\mathrm{D}}^{20}:-152.3(c=$ 1.00, $\mathrm{CHCl}_{3}$ ); Chiral HPLC: Chiralpak ID, 4.6 × $250 \mathrm{~mm} ; 20 \%$ i-PrOH/hexane, $1.0 \mathrm{~mL} / \mathrm{min}, 254 \mathrm{~nm}$; $\operatorname{tr}($ minor $)=14.9 \mathrm{~min}, \operatorname{tr}($ major $)=16.6 \mathrm{~min}, 99.3 / 0.7 \mathrm{er}$.

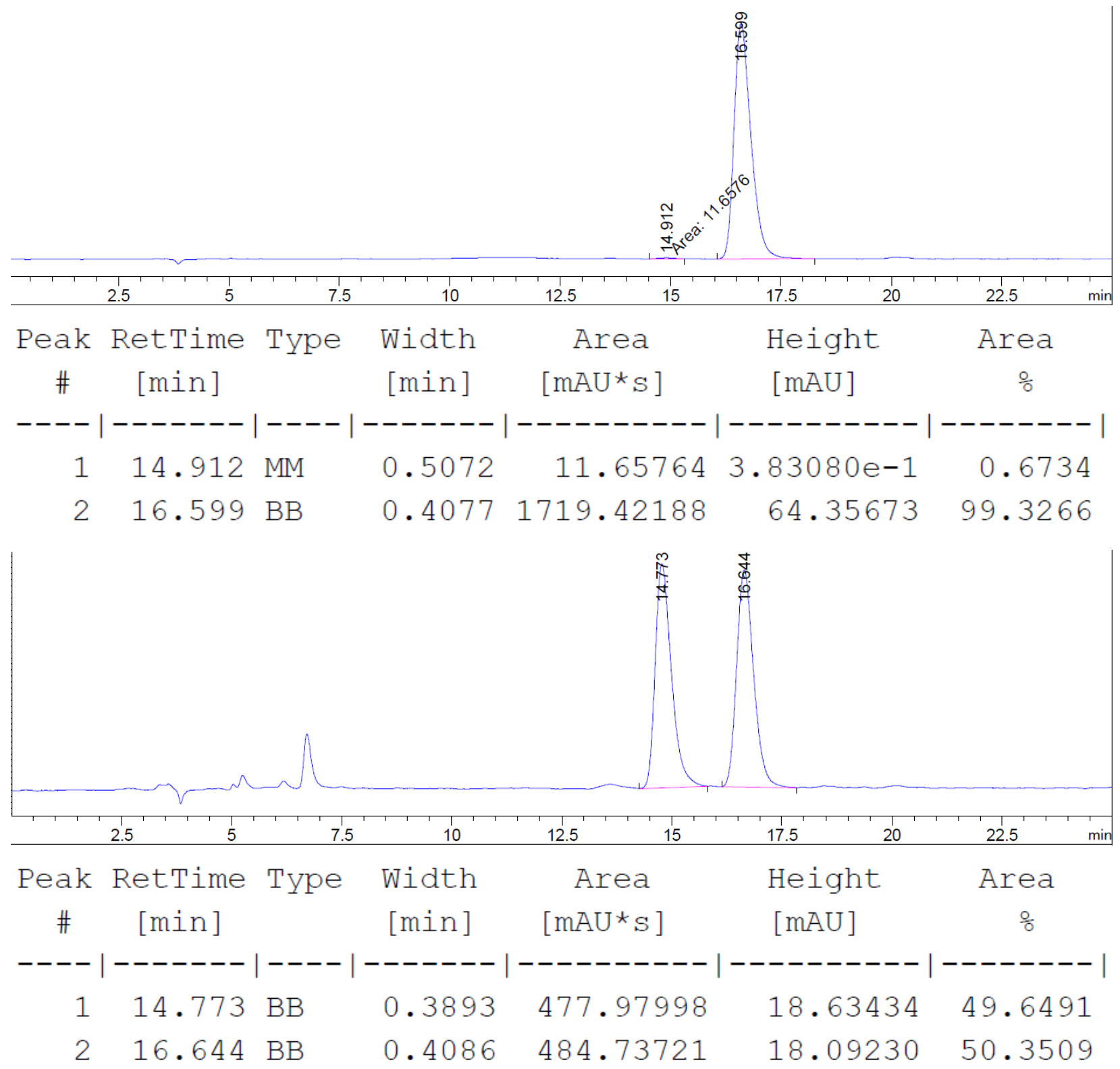




\section{(-)-(S)-3-(4-methoxyphenyl)-3,4-dihydroisoquinolin-1(2H)-one (3ab)}

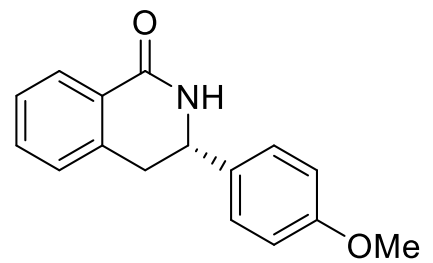

General Procedure A. Yield: $87 \%$ (44.0 mg, $174 \mu \mathrm{mol})$, white solid. The spectra match with the literature values ${ }^{9} .{ }^{1} \mathrm{H}$ NMR $\left(400 \mathrm{MHz}, \mathrm{CDCl}_{3}\right) \delta$ $=8.11(\mathrm{dd}, J=7.6,1.4 \mathrm{~Hz}, 1 \mathrm{H}), 7.46(\mathrm{td}, J=7.5,1.5 \mathrm{~Hz}, 1 \mathrm{H}), 7.37$ (t, $J=7.5 \mathrm{~Hz}, 1 \mathrm{H}), 7.32(\mathrm{~d}, J=8.7 \mathrm{~Hz}, 2 \mathrm{H}), 7.19(\mathrm{~d}, J=7.4 \mathrm{~Hz}, 1 \mathrm{H}), 6.91$ (d, $J=8.6 \mathrm{~Hz}, 2 \mathrm{H}), 6.02(\mathrm{~s}, 1 \mathrm{H}), 4.81(\mathrm{dd}, J=11.4,4.5 \mathrm{~Hz}, 1 \mathrm{H}), 3.82$ (s, 3H), 3.19 (dd, $J=15.7,11.4 \mathrm{~Hz}, 1 \mathrm{H}$ ), 3.07 (dd, $J=15.7,4.5 \mathrm{~Hz}, 1 \mathrm{H}$ ) ppm; $\boldsymbol{R}_{\mathrm{f}}: 0.27$ (hexane/EtOAc $=1 / 1)$; $[\alpha]_{\mathrm{D}}^{20}:-108.2\left(c=1.00, \mathrm{CHCl}_{3}\right)$; Chiral HPLC: Chiralpak IB, 4.6 x $250 \mathrm{~mm}$; $20 \% \mathrm{i}-\mathrm{PrOH} / \mathrm{hexane}, 1.0 \mathrm{~mL} / \mathrm{min}, 266 \mathrm{~nm}$; $\operatorname{tr}$ (major) $=11.9 \mathrm{~min}, \operatorname{tr}(\operatorname{minor})=13.4 \mathrm{~min}, 99.5 / 0.5 \mathrm{er}$.

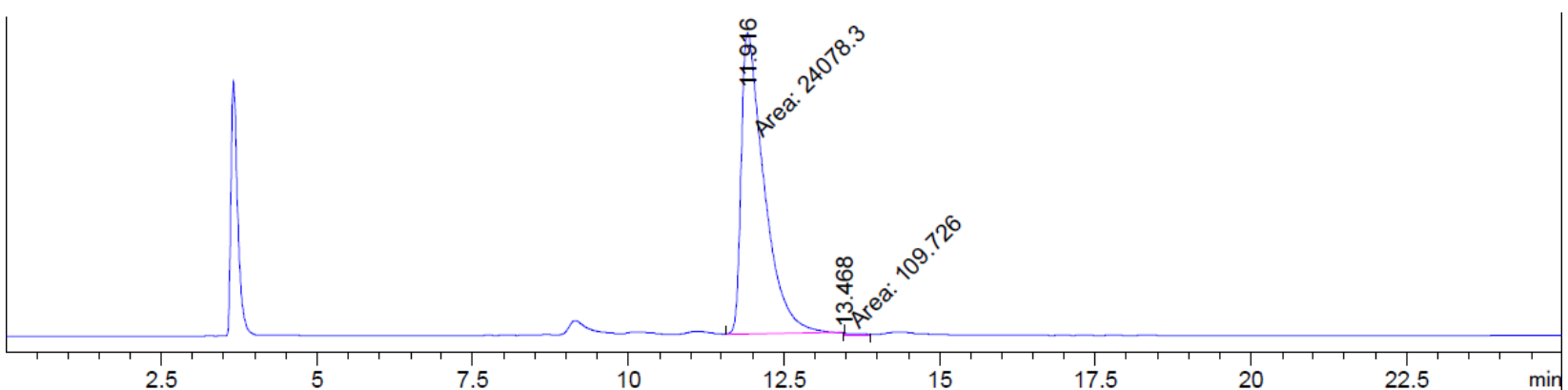

\begin{tabular}{|c|c|c|c|c|c|c|}
\hline $\begin{array}{l}e a k \\
\#\end{array}$ & $\begin{array}{c}\text { RetTime } \\
\text { [min] }\end{array}$ & Type & $\begin{array}{l}\text { Width } \\
\text { [min] }\end{array}$ & $\begin{array}{c}\text { Area } \\
{\left[\mathrm{mAU}^{*} \mathrm{~S}\right]}\end{array}$ & $\begin{array}{l}\text { Height } \\
{[\mathrm{mAU}]}\end{array}$ & $\begin{array}{c}\text { Area } \\
\frac{8}{8}\end{array}$ \\
\hline & & & & & ----- & \\
\hline 1 & & MM & & $2.40783 e 4$ & 46393 & 99 \\
\hline 2 & .468 & MM & 65 & 109.72580 & 6.04914 & 536 \\
\hline
\end{tabular}




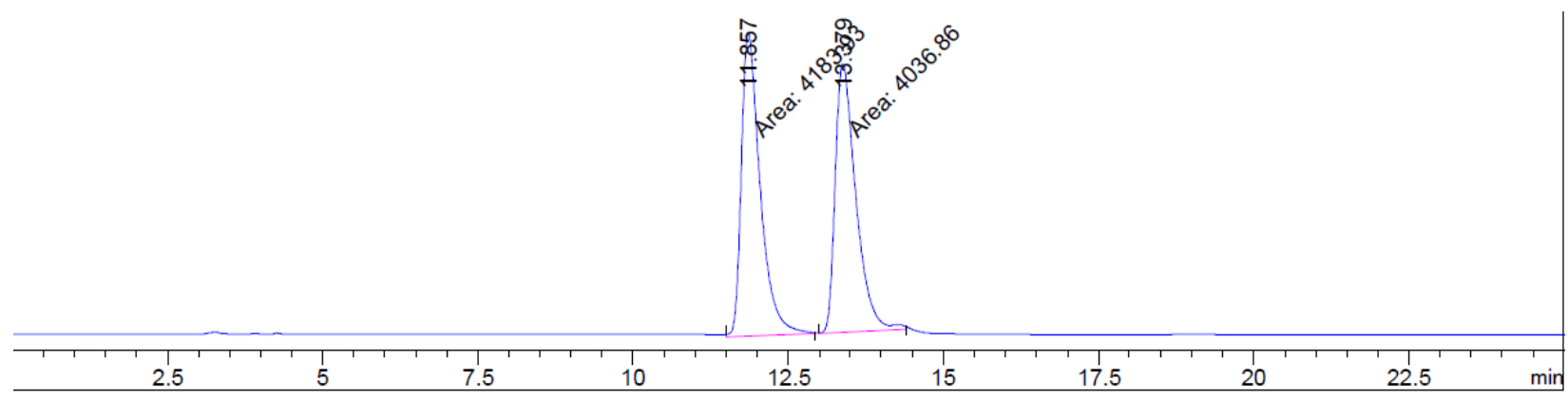

\begin{tabular}{|c|c|c|c|c|c|c|}
\hline $\begin{array}{c}\text { eak } \\
\#\end{array}$ & $\begin{array}{c}\text { RetTime } \\
\text { [min] }\end{array}$ & Type & $\begin{array}{l}\text { Width } \\
\text { [min] }\end{array}$ & $\begin{array}{c}\text { Area } \\
{\left[\mathrm{mAU}^{*} \mathrm{~s}\right]}\end{array}$ & $\begin{array}{l}\text { Height } \\
{[\mathrm{mAU}]}\end{array}$ & $\begin{array}{c}\text { Area } \\
\frac{8}{8}\end{array}$ \\
\hline & & & & ------ & ---1 & \\
\hline 1 & & & & 4183. & 22 & 5 \\
\hline 2 & 379 & & 854 & 4036.86328 & .55865 & 055 \\
\hline
\end{tabular}

(-)-(S)-3-(4-(tert-butyl)phenyl)-3,4-dihydroisoquinolin-1(2H)-one (3ac)

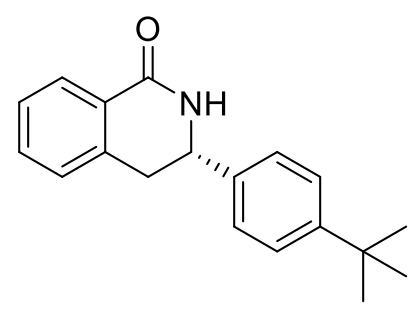

General Procedure A. Yield: 81\% (45.0 mg, $161 \mu \mathrm{mol})$, white solid. The spectra match with the literature values ${ }^{9} .{ }^{1} \mathbf{H}$ NMR $\left(400 \mathrm{MHz}, \mathrm{CDCl}_{3}\right) \delta$ $=8.13(\mathrm{dd}, J=7.7,1.4 \mathrm{~Hz}, 1 \mathrm{H}), 7.50-7.45(\mathrm{~m}, 1 \mathrm{H}), 7.44-7.31(\mathrm{~m}$, $5 \mathrm{H}), 7.19(\mathrm{~d}, J=7.6 \mathrm{~Hz}, 1 \mathrm{H}), 5.98(\mathrm{~s}, 1 \mathrm{H}), 4.84(\mathrm{dd}, J=11.4,4.6 \mathrm{~Hz}$, $1 \mathrm{H}), 3.21(\mathrm{dd}, J=15.7,11.4 \mathrm{~Hz}, 1 \mathrm{H}), 3.13-2.99(\mathrm{~m}, 1 \mathrm{H}), 1.33(\mathrm{~s}, 9 \mathrm{H})$ ppm; $\boldsymbol{R}_{\mathbf{f}}: 0.23($ hexane/EtOAc $=1 / 1) ;[\boldsymbol{\alpha}]_{D}^{20}:-111.7\left(c=1.00, \mathrm{CHCl}_{3}\right)$; Chiral HPLC: Chiralpak IA, $4.6 \times 250 \mathrm{~mm} ; 20 \% \mathrm{i}-\mathrm{PrOH} / \mathrm{hexane}, 1.0 \mathrm{~mL} / \mathrm{min}, 254 \mathrm{~nm}$; $\operatorname{tr}$ (minor) = $7.1 \mathrm{~min}, \operatorname{tr}$ (major) = $8.5 \mathrm{~min}$, $98.1 / 1.9 \mathrm{er}$. 


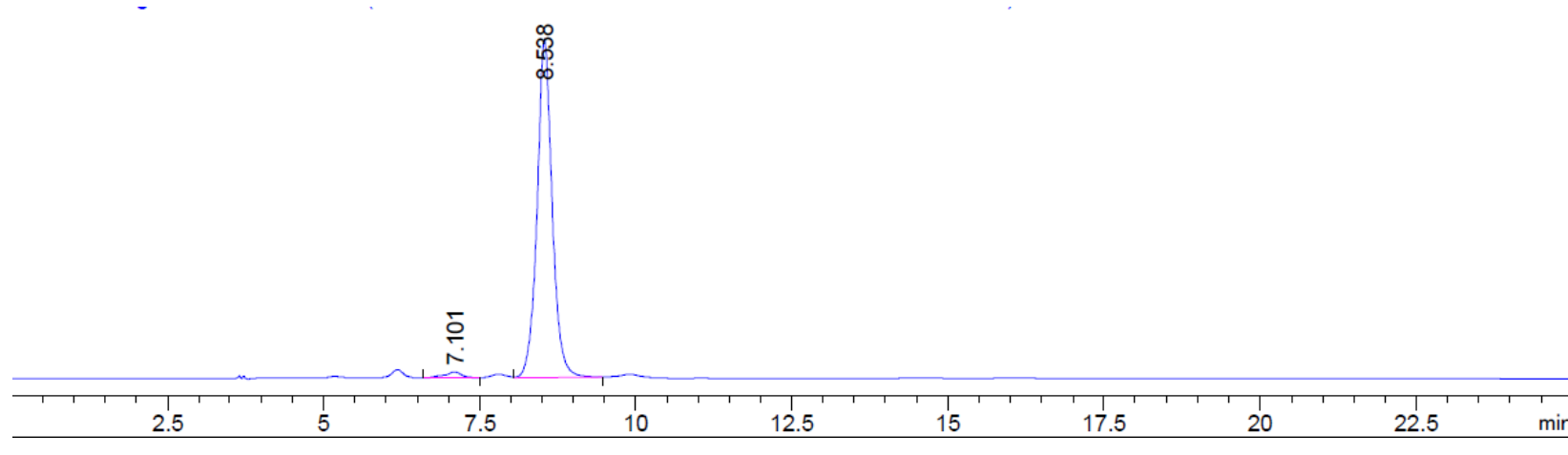

\begin{tabular}{|c|c|c|c|c|c|c|}
\hline $\begin{array}{c}\text { eak } \\
\text { \# }\end{array}$ & $\begin{array}{c}\text { RetTime } \\
\text { [min] }\end{array}$ & Type & $\begin{array}{l}\text { Width } \\
\text { [min] }\end{array}$ & $\begin{array}{c}\text { Area } \\
{\left[\mathrm{mAU}^{*} \mathrm{~S}\right]}\end{array}$ & $\begin{array}{l}\text { Height } \\
{[\mathrm{mAU}]}\end{array}$ & $\begin{array}{c}\text { Area } \\
\frac{8}{8}\end{array}$ \\
\hline & & & & & & \\
\hline 1 & & & & 107 & 5.74767 & 9387 \\
\hline & 538 & & & 5429.61279 & 322.10098 & .0613 \\
\hline
\end{tabular}

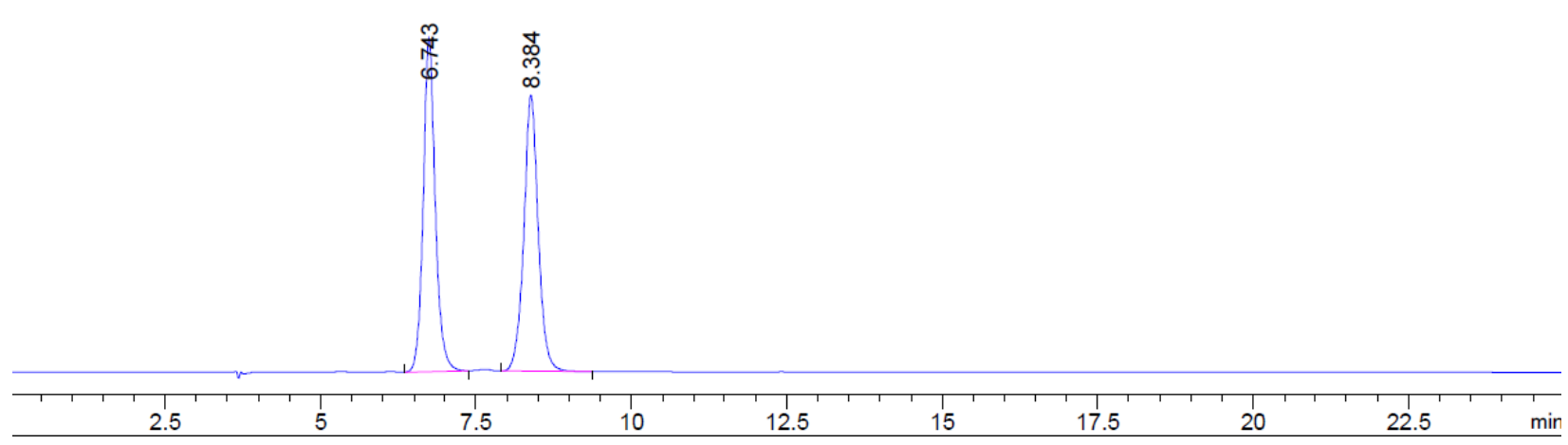

Peak RetTime Type Width Area Height Area

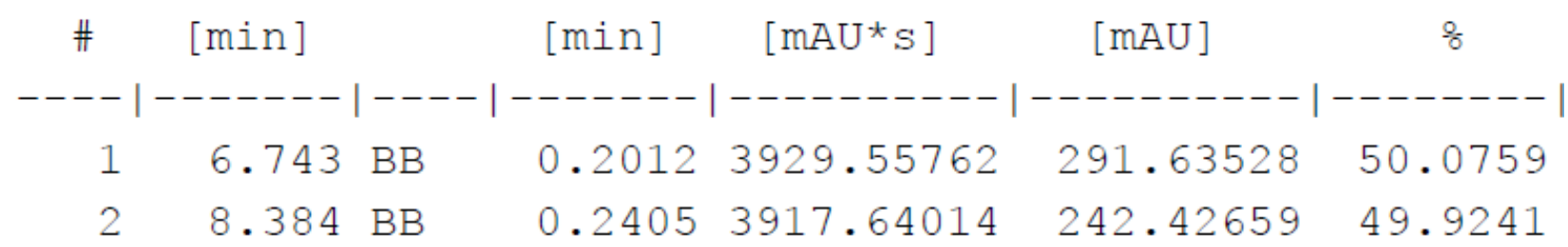

\section{$(-)-(S)-3-(p$-tolyl)-3,4-dihydroisoquinolin-1(2H)-one (3ad)}

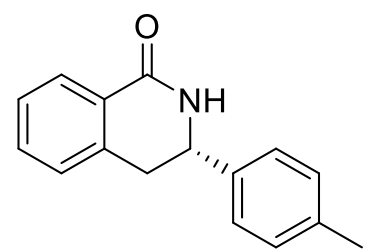

General Procedure A. Yield: $88 \%$ (42.0 mg, $177 \mu \mathrm{mol})$, white solid. The spectra match with the literature values ${ }^{9} .{ }^{1} \mathrm{H}$ NMR $\left(400 \mathrm{MHz}, \mathrm{CDCl}_{3}\right) \delta=$ 8.12 (dd, $J=7.8,1.4 \mathrm{~Hz}, 1 \mathrm{H}$ ), 7.46 (td, $J=7.5,1.5 \mathrm{~Hz}, 1 \mathrm{H}$ ), 7.37 (t, $J=7.5$ $\mathrm{Hz}, 1 \mathrm{H}), 7.29(\mathrm{~d}, J=8.1 \mathrm{~Hz}, 2 \mathrm{H}), 7.22-7.16(\mathrm{~m}, 3 \mathrm{H}), 6.08(\mathrm{~s}, 1 \mathrm{H}), 4.82$ (dd, $J=11.2,4.7 \mathrm{~Hz}, 1 \mathrm{H}$ ), 3.19 (dd, $J=15.7,11.2 \mathrm{~Hz}, 1 \mathrm{H}$ ), 3.09 (dd, $J=15.7,4.7 \mathrm{~Hz}, 1 \mathrm{H}), 2.36$ $(\mathrm{s}, 3 \mathrm{H}) \mathrm{ppm} ; \boldsymbol{R}_{\mathrm{f}}: 0.42($ hexane/EtOAc $=1 / 1)$; $[\alpha]_{\mathrm{D}}^{20}:-154.5\left(\mathrm{c}=1.00, \mathrm{CHCl}_{3}\right)$; Chiral HPLC: 
Chiralpak ID, 4.6 x $250 \mathrm{~mm}$; 20\% i-PrOH/hexane, $1.0 \mathrm{~mL} / \mathrm{min}, 254 \mathrm{~nm}$; (minor) = $17.3 \mathrm{~min}$, tr (major) $=24.8 \mathrm{~min}, \operatorname{tr} 99.5 / 0.5 \mathrm{er}$.

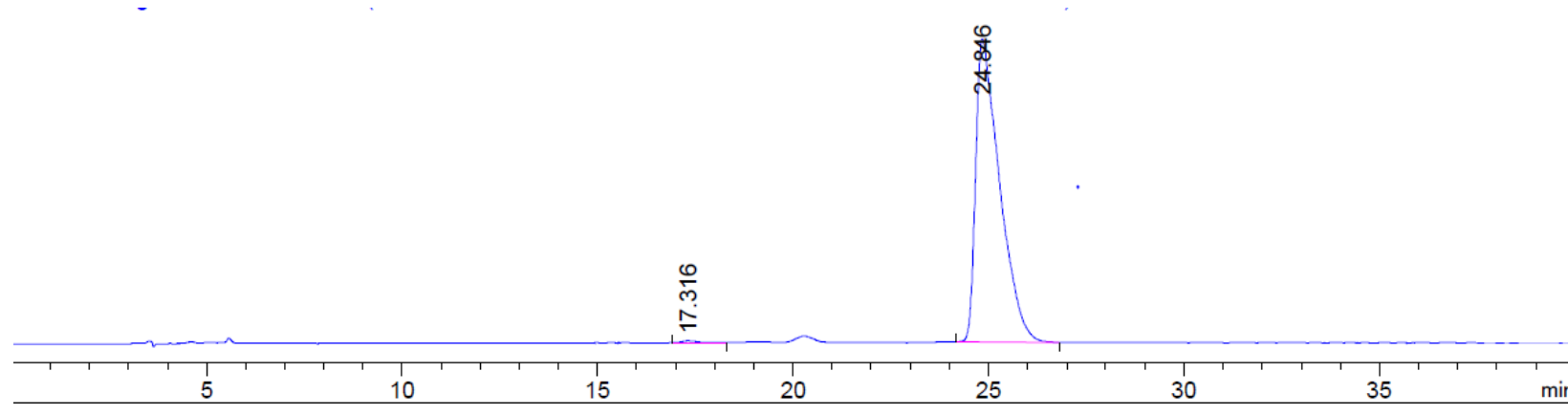

\begin{tabular}{|c|c|c|c|c|c|c|}
\hline $\begin{array}{c}\text { Peak } \\
\quad \#\end{array}$ & $\begin{array}{c}\text { RetTime } \\
\text { [min] }\end{array}$ & Type & $\begin{array}{l}\text { Width } \\
\text { [min] }\end{array}$ & $\begin{array}{c}\text { Area } \\
{\left[\mathrm{mAU}^{*} \mathrm{~s}\right]}\end{array}$ & $\begin{array}{l}\text { Height } \\
{[\mathrm{mAU}]}\end{array}$ & $\begin{array}{c}\text { Area } \\
\frac{\circ}{8}\end{array}$ \\
\hline & & & & 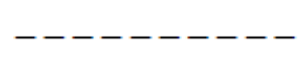 & & \\
\hline 1 & 17.316 & $\mathrm{BB}$ & 0.3738 & 50.18780 & 1.73348 & 890 \\
\hline 2 & 24.846 & $\mathrm{BB}$ & 0.5871 & $1.02127 e 4$ & 239.68126 & 5110 \\
\hline
\end{tabular}

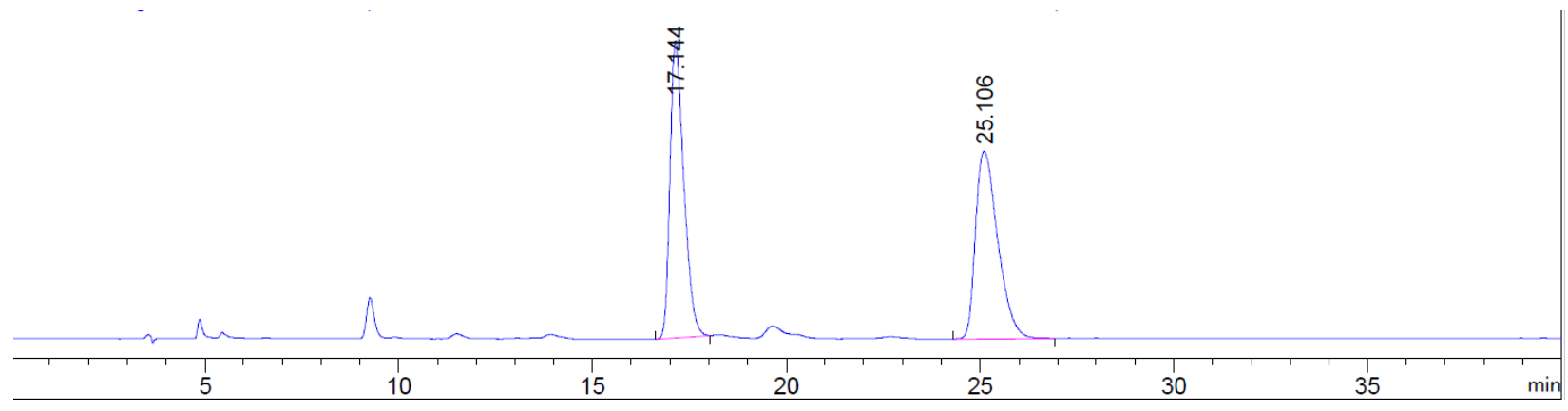

\begin{tabular}{|c|c|c|c|c|c|c|}
\hline $\begin{array}{c}\text { Peak } \\
\#\end{array}$ & $\begin{array}{c}\text { RetTime } \\
\text { [min] }\end{array}$ & Type & $\begin{array}{l}\text { Width } \\
\text { [min] }\end{array}$ & $\begin{array}{c}\text { Area } \\
{\left[\mathrm{mAU}^{*} \mathrm{~s}\right]}\end{array}$ & $\begin{array}{l}\text { Height } \\
\text { [mAU] }\end{array}$ & $\begin{array}{c}\text { Area } \\
\frac{\circ}{\circ}\end{array}$ \\
\hline & & & & & & \\
\hline 1 & 17.144 & $\mathrm{BB}$ & 0.3700 & 3993.09424 & 161.77075 & 49. \\
\hline 2 & 25.106 & $\mathrm{BB}$ & 0.6087 & 4087.70972 & .17645 & 50 \\
\hline
\end{tabular}

\section{$(-)-(S)-3-(m$-tolyl)-3,4-dihydroisoquinolin-1(2H)-one (3ae)}

General Procedure A. Yield: $88 \%(42.0 \mathrm{mg}, 177 \mu \mathrm{mol})$, pale yellow solid.
The spectra match with the literature values ${ }^{9} .{ }^{1} \mathbf{H}$ NMR $\left(400 \mathrm{MHz}, \mathrm{CDCl}_{3}\right)$
$\delta=8.12(\mathrm{dd}, J=7.8,1.4 \mathrm{~Hz}, 1 \mathrm{H}), 7.46(\mathrm{td}, J=7.4,1.5 \mathrm{~Hz}, 1 \mathrm{H}), 7.41-$
$7.34(\mathrm{~m}, 1 \mathrm{H}), 7.28(\mathrm{t}, J=7.5 \mathrm{~Hz}, 1 \mathrm{H}), 7.23-7.12(\mathrm{~m}, 4 \mathrm{H}), 6.11(\mathrm{~s}, 1 \mathrm{H})$, 4.82 (dd, $J=11.3,4.7 \mathrm{~Hz}, 1 \mathrm{H}$ ), 3.20 (dd, $J=15.7,11.2 \mathrm{~Hz}, 1 \mathrm{H}), 3.10$ (dd, $J=15.8,4.7 \mathrm{~Hz}, 1 \mathrm{H}$ ), 
$2.37(\mathrm{~s}, 3 \mathrm{H}) \mathrm{ppm} ; \boldsymbol{R}_{\mathrm{f}}: 0.53($ hexane/EtOAc $=1 / 1) ;[\boldsymbol{\alpha}]_{\mathrm{D}}^{20}:-154.8\left(c=1.00, \mathrm{CHCl}_{3}\right)$; Chiral HPLC: Chiralpak IC, $4.6 \times 250 \mathrm{~mm} ; 20 \% \mathrm{i}-\mathrm{PrOH} /$ hexane, $1.0 \mathrm{~mL} / \mathrm{min}, 254 \mathrm{~nm}$; $\operatorname{tr}$ (major) = $15.6 \mathrm{~min}$, tr $(\operatorname{minor})=17.8 \mathrm{~min}, 99.6 / 0.4 \mathrm{er}$.

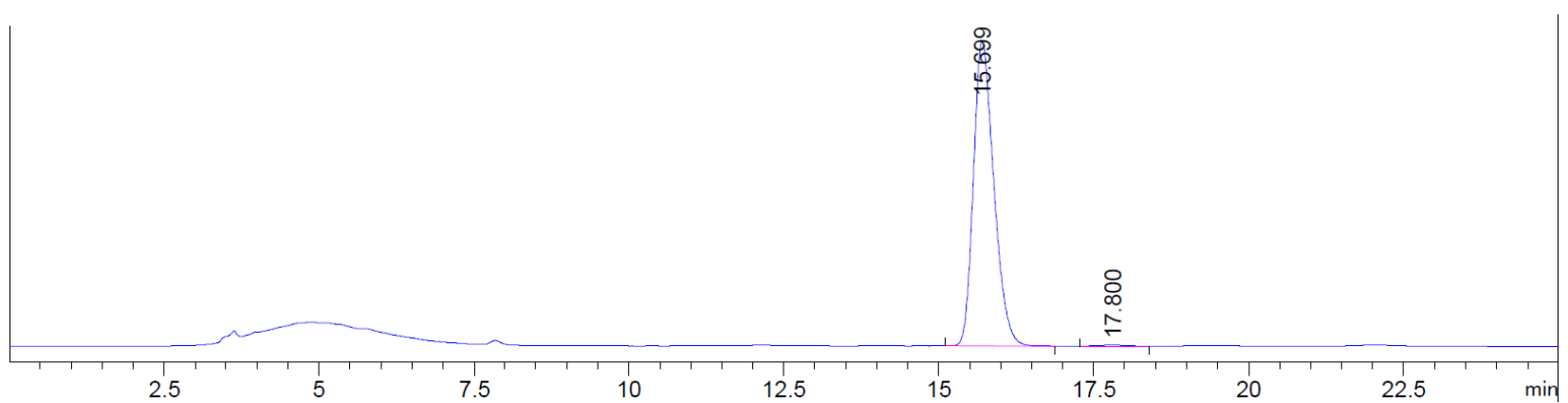

Peak RetTime Type Width Area Height Area

\begin{tabular}{|c|c|c|c|c|c|}
\hline \# & {$[\mathrm{min}]$} & [min] & {$\left[\mathrm{mAU}^{*} \mathrm{~s}\right]$} & [mAU ] & $\frac{\circ}{0}$ \\
\hline & & & & & \\
\hline 1 & $15.699 \mathrm{BB}$ & & 1.11487 & 486. & \\
\hline 2 & $17.800 \mathrm{BB}$ & 0.3144 & 43.74466 & 1.68782 & \\
\hline
\end{tabular}

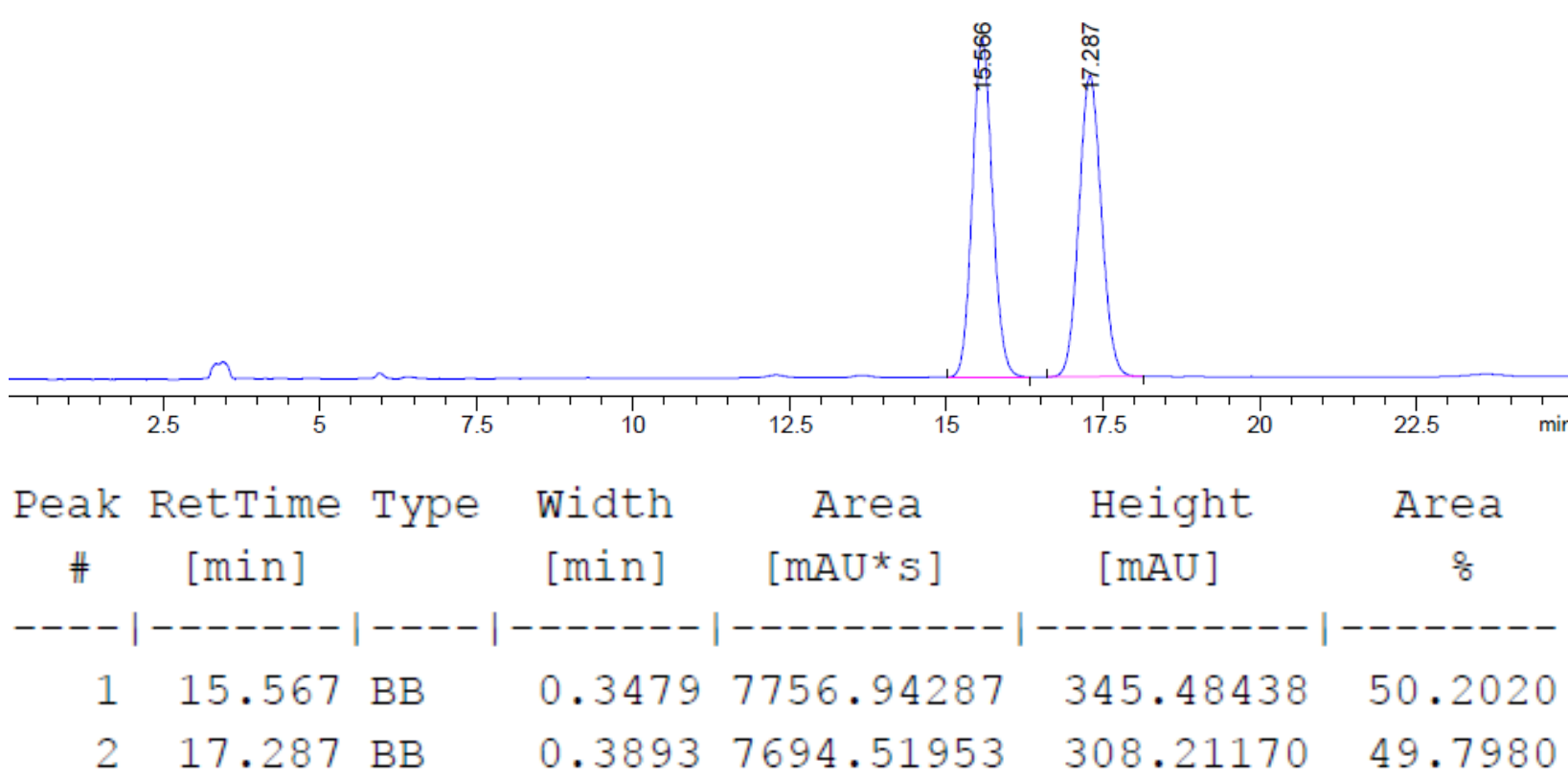


(-)-(S)-3-(4-fluorophenyl)-3,4-dihydroisoquinolin-1(2H)-one (3af)<smiles>O=C1N[C@H](c2ccc(F)cc2)Cc2ccccc21</smiles>

General Procedure A. Yield: 85\% (41.0 mg, $170 \mu \mathrm{mol})$, white solid. The spectra match with the literature values ${ }^{9} .{ }^{1} \mathrm{H}$ NMR $\left(400 \mathrm{MHz}, \mathrm{CDCl}_{3}\right) \delta=$ 8.11 (dd, $J=7.8,1.4 \mathrm{~Hz}, 1 \mathrm{H}$ ), 7.47 (td, $J=7.5,1.4 \mathrm{~Hz}, 1 \mathrm{H}$ ), $7.41-7.34$ $(\mathrm{m}, 3 \mathrm{H}), 7.18(\mathrm{~d}, J=7.5 \mathrm{~Hz}, 1 \mathrm{H}), 7.11-7.01(\mathrm{~m}, 2 \mathrm{H}), 6.13(\mathrm{~s}, 1 \mathrm{H}), 4.86$ (dd, $J=10.3,5.3 \mathrm{~Hz}, 1 \mathrm{H}), 3.24-3.07(\mathrm{~m}, 2 \mathrm{H}) \mathrm{ppm} ;{ }^{19} \mathrm{~F}\left\{{ }^{1} \mathrm{H}\right\}$ NMR $(376$ $\left.\mathrm{MHz}, \mathrm{CDCl}_{3}\right) \delta=-113.4 \mathrm{ppm} ; \boldsymbol{R}_{\mathrm{f}}: 0.37$ (hexane/EtOAc = 1/1); $[\alpha]_{\mathrm{D}}^{20}:-164.8\left(c=1.00, \mathrm{CHCl}_{3}\right)$; Chiral HPLC: Chiralpak IA, $4.6 \times 250 \mathrm{~mm} ; 20 \% \mathrm{i}-\mathrm{PrOH} /$ hexane, $1.0 \mathrm{~mL} / \mathrm{min}, 254 \mathrm{~nm}$; tr (minor) = $9.3 \mathrm{~min}, \operatorname{tr}$ (major) $=10.8 \mathrm{~min}, 99.5 / 0.5 \mathrm{er}$.

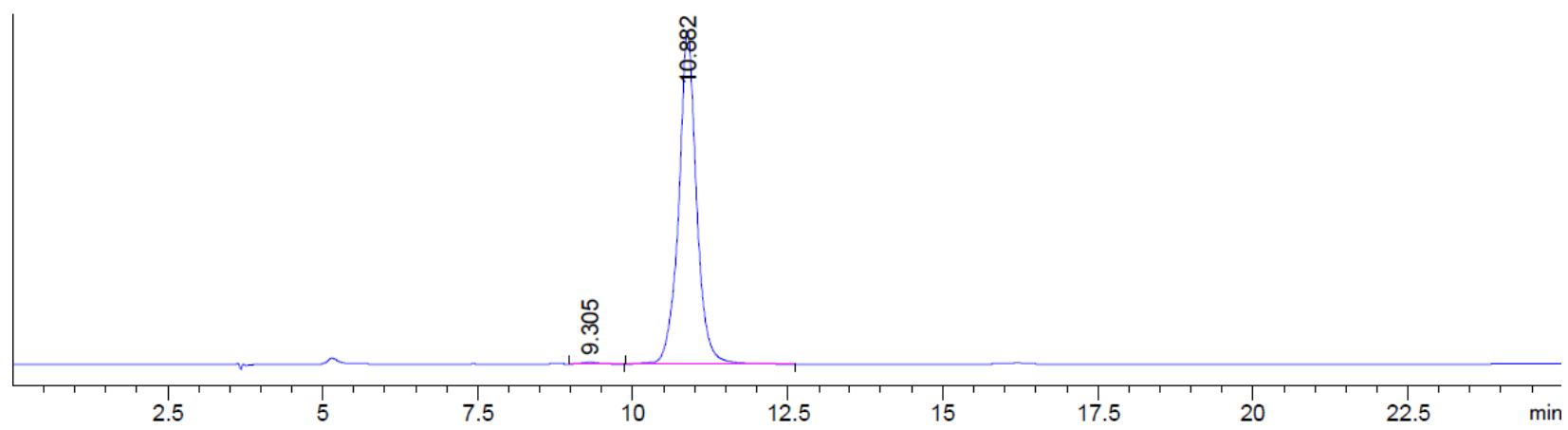

Peak RetTime Type Width Area Height Area $\begin{array}{lllll}\# & {[\mathrm{~min}]} & {[\mathrm{min}]} & {\left[\mathrm{mAU}{ }^{*} \mathrm{~s}\right]} & {[\mathrm{mAU}]}\end{array}$ $----|-------|----|-------|----------|----------|--------\mid$

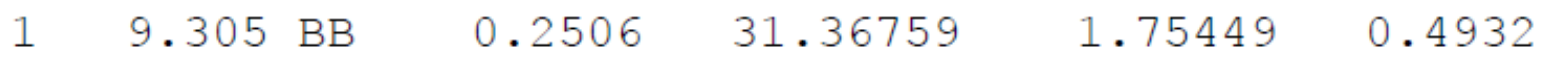
$\begin{array}{lllllll}2 & 10.882 & \mathrm{BB} & 0.2941 & 6328.47021 & 315.36829 & 99.5068\end{array}$

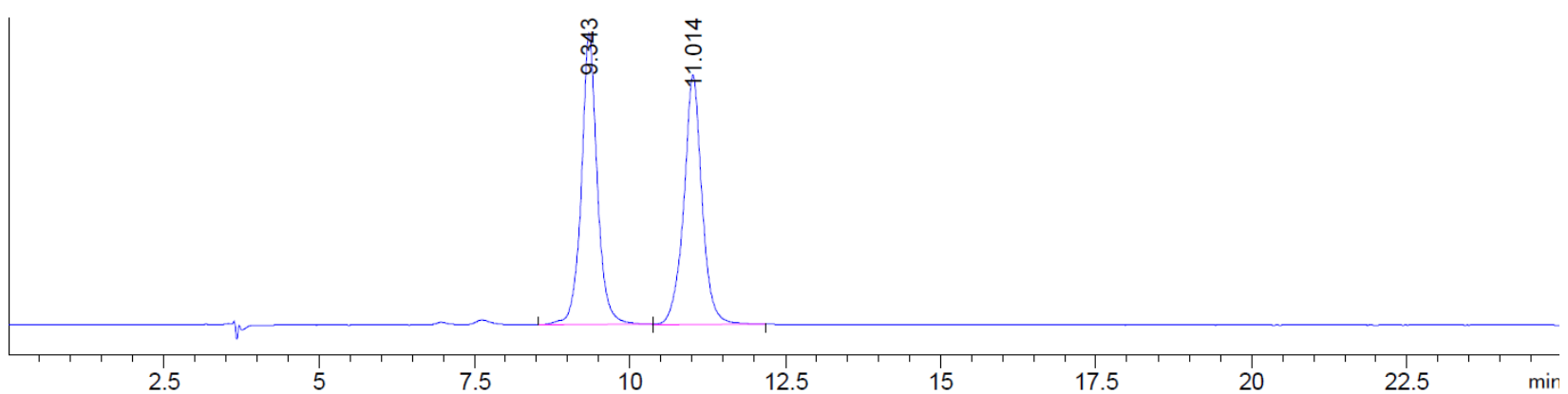

Peak RetTime Type Width Area Height Area

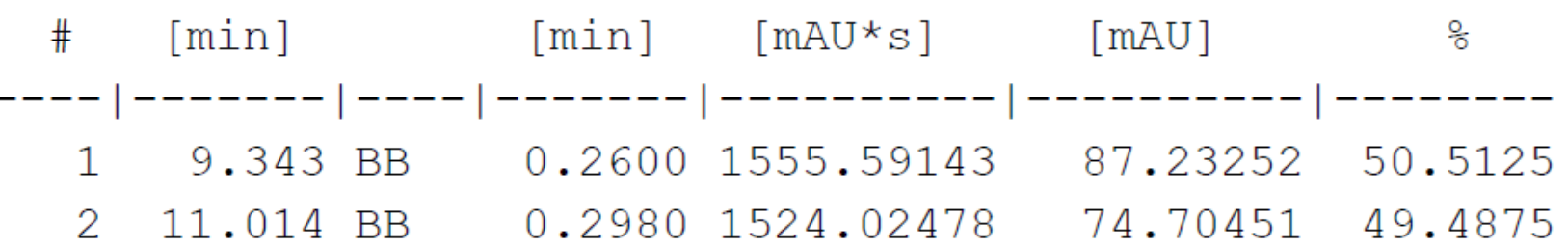




\section{(-)-methyl (S)-1-oxo-1,2,3,4-tetrahydroisoquinoline-3-carboxylate (3ag)}<smiles>COC(=O)[C@@H]1Cc2ccccc2C(=O)N1</smiles>

General Procedure C. Yield: 68\% (28.0 mg, $136 \mu \mathrm{mol})$, white solid. The spectra match with the literature values. ${ }^{8}{ }^{1} \mathrm{H}$ NMR $\left(400 \mathrm{MHz}, \mathrm{CDCl}_{3}\right) \delta=8.08$ (dd, $J=7.7,1.4 \mathrm{~Hz}, 1 \mathrm{H}), 7.47(\mathrm{td}, J=7.5,1.5 \mathrm{~Hz}, 1 \mathrm{H}), 7.41-7.34(\mathrm{~m}, 1 \mathrm{H})$, 7.24 (d, $J=7.5 \mathrm{~Hz}, 1 \mathrm{H}$ ), 6.48 (s, 1H), 4.41 (ddd, $J=10.1,5.2,2.0 \mathrm{~Hz}, 1 \mathrm{H}$ ), 3.80 (s, 3H), 3.33 (dd, $J=15.7,5.1 \mathrm{~Hz}, 1 \mathrm{H}$ ), 3.21 (dd, $J=15.7,10.0 \mathrm{~Hz}, 1 \mathrm{H}$ ) ppm; $\boldsymbol{R}_{\mathrm{f}}: 0.17$ (hexane/EtOAc = 1/1); $[\alpha]_{D}^{20}:-62.6\left(c=1.00, \mathrm{CHCl}_{3}\right)$; Chiral HPLC: Chiralpak IA, 4.6 x 250 mm; $20 \% \mathrm{i}-\mathrm{PrOH} /$ hexane, $1.0 \mathrm{~mL} / \mathrm{min}, 254 \mathrm{~nm}$; $\operatorname{tr}$ (major) = $10.7 \mathrm{~min}$, $\operatorname{tr}$ (minor) = 11.9 min, 90.8/9.2 er.

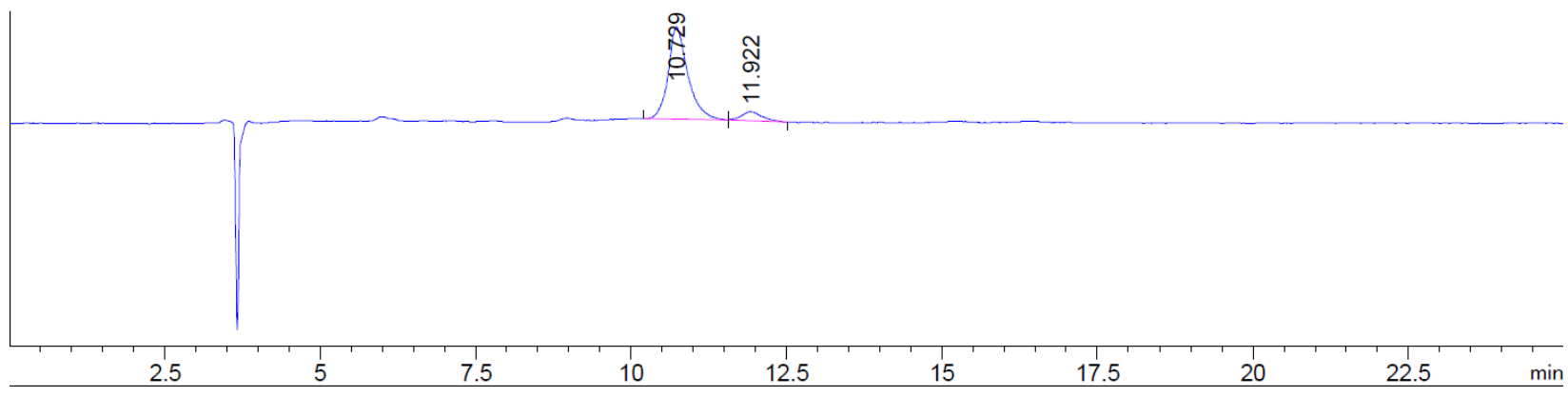

\begin{tabular}{|c|c|c|c|c|c|c|}
\hline $\begin{array}{c}\text { Peak } \\
\quad \#\end{array}$ & $\begin{array}{c}\text { RetTime } \\
\text { [min] }\end{array}$ & Type & $\begin{array}{l}\text { Width } \\
\text { [min] }\end{array}$ & $\begin{array}{c}\text { Area } \\
{\left[\mathrm{mAU}^{*} \mathrm{~s}\right]}\end{array}$ & $\begin{array}{l}\text { Height } \\
{[\mathrm{mAU}]}\end{array}$ & $\begin{array}{c}\text { Area } \\
\text { 응 }\end{array}$ \\
\hline & & & & -------- & ------- & \\
\hline 1 & 10.729 & $\mathrm{BB}$ & 0.3204 & 336.58124 & 15.31702 & \\
\hline 2 & 11.922 & $\mathrm{BB}$ & 0.3041 & 33.95538 & 1.48872 & \\
\hline
\end{tabular}

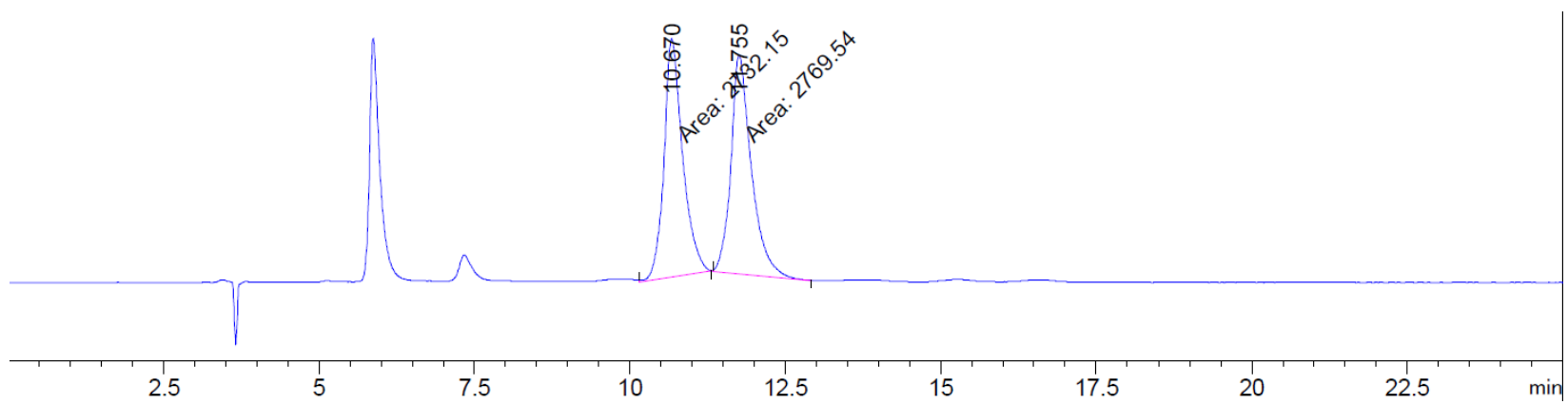

Peak RetTime Type Width Area Height Area

\begin{tabular}{ccccccc}
$\#$ & {$[\mathrm{~min}]$} & {$[\mathrm{min}]$} & {$\left[\mathrm{mAU}{ }^{*} \mathrm{~s}\right]$} & {$[\mathrm{mAU}]$} & \% \\
\hline 1 & 10.670 & $\mathrm{MM}$ & 0.3478 & 2732.14868 & 130.94331 & 49.6601 \\
2 & 11.755 & $\mathrm{MM}$ & 0.3824 & 2769.54419 & 120.70953 & 50.3399
\end{tabular}


(-)-tert-butyl (S)-1-oxo-1,2,3,4-tetrahydroisoquinoline-3-carboxylate (3ah)

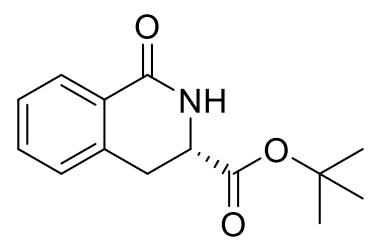

General Procedure A. Yield: $75 \%(37.0 \mathrm{mg}, 150 \mu \mathrm{mol})$, white solid. ${ }^{1} \mathrm{H}$ NMR $\left(400 \mathrm{MHz}, \mathrm{CDCl}_{3}\right) \delta=8.07(\mathrm{dd}, J=7.7,1.4 \mathrm{~Hz}, 1 \mathrm{H}), 7.46$ (td, $J=$ $7.5,1.5 \mathrm{~Hz}, 1 \mathrm{H}), 7.37$ (t, $J=7.5 \mathrm{~Hz}, 1 \mathrm{H}), 7.24(\mathrm{~d}, J=7.5 \mathrm{~Hz}, 1 \mathrm{H}), 6.35$ (s, $1 \mathrm{H}$ ), 4.27 (ddd, $J=10.7,4.9,1.7 \mathrm{~Hz}, 1 \mathrm{H}$ ), 3.26 (dd, $J=15.7,5.0 \mathrm{~Hz}, 1 \mathrm{H}$ ), $3.14(\mathrm{dd}, J=15.6,10.7 \mathrm{~Hz}, 1 \mathrm{H}), 1.47(\mathrm{~s}, 9 \mathrm{H}) \mathrm{ppm} ;{ }^{13} \mathrm{C}\left\{{ }^{1} \mathrm{H}\right\}$ NMR $\left(101 \mathrm{MHz}, \mathrm{CDCl}_{3}\right) \delta=169.4$, 165.3, 136.6, 132.6, 128.7, 128.3, 127.6, 127.5, 83.3, 53.8, 31.7, 28.1 ppm; IR (ATR): 3225, 2976, 2930, 1731, 1669, 1605, 1464, 1392, 1368, 1323, 1310, 1244, 1148, 1007, 842, 742, $568 \mathrm{~cm}^{-1}$; HRMS (ESI): calcd. for $\left[\mathrm{C}_{14} \mathrm{H}_{18} \mathrm{NO}_{3}\right]^{+}, \quad\left[\mathrm{M}+\mathrm{H}^{+}\right]^{+}$: 248.1281; found: 248.1284; $\boldsymbol{R}_{\mathrm{f}}: 0.36$ (hexane/EtOAc $=1 / 1) ;[\alpha]_{D}^{20}:-75.5\left(c=1.00, \mathrm{CHCl}_{3}\right)$; Chiral HPLC: Chiralpak ID, 4.6 x $250 \mathrm{~mm}$; $20 \% \mathrm{i}-\mathrm{PrOH} /$ hexane, $1.0 \mathrm{~mL} / \mathrm{min}, 274 \mathrm{~nm}$; $\operatorname{tr}$ (major) $=18.7 \mathrm{~min}, \operatorname{tr}(\operatorname{minor})=23.3 \mathrm{~min}, 98.9 / 1.1 \mathrm{er}$.

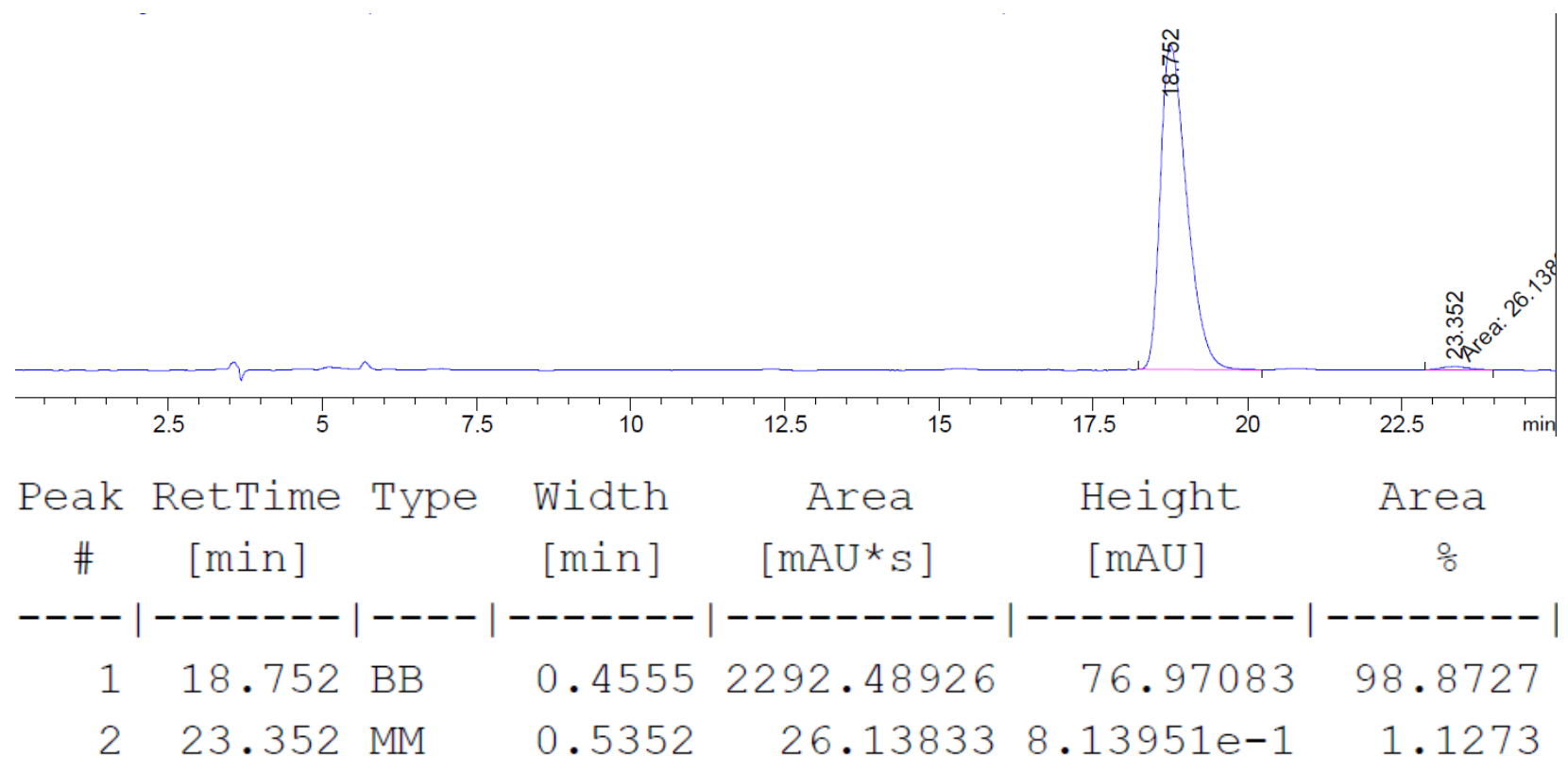




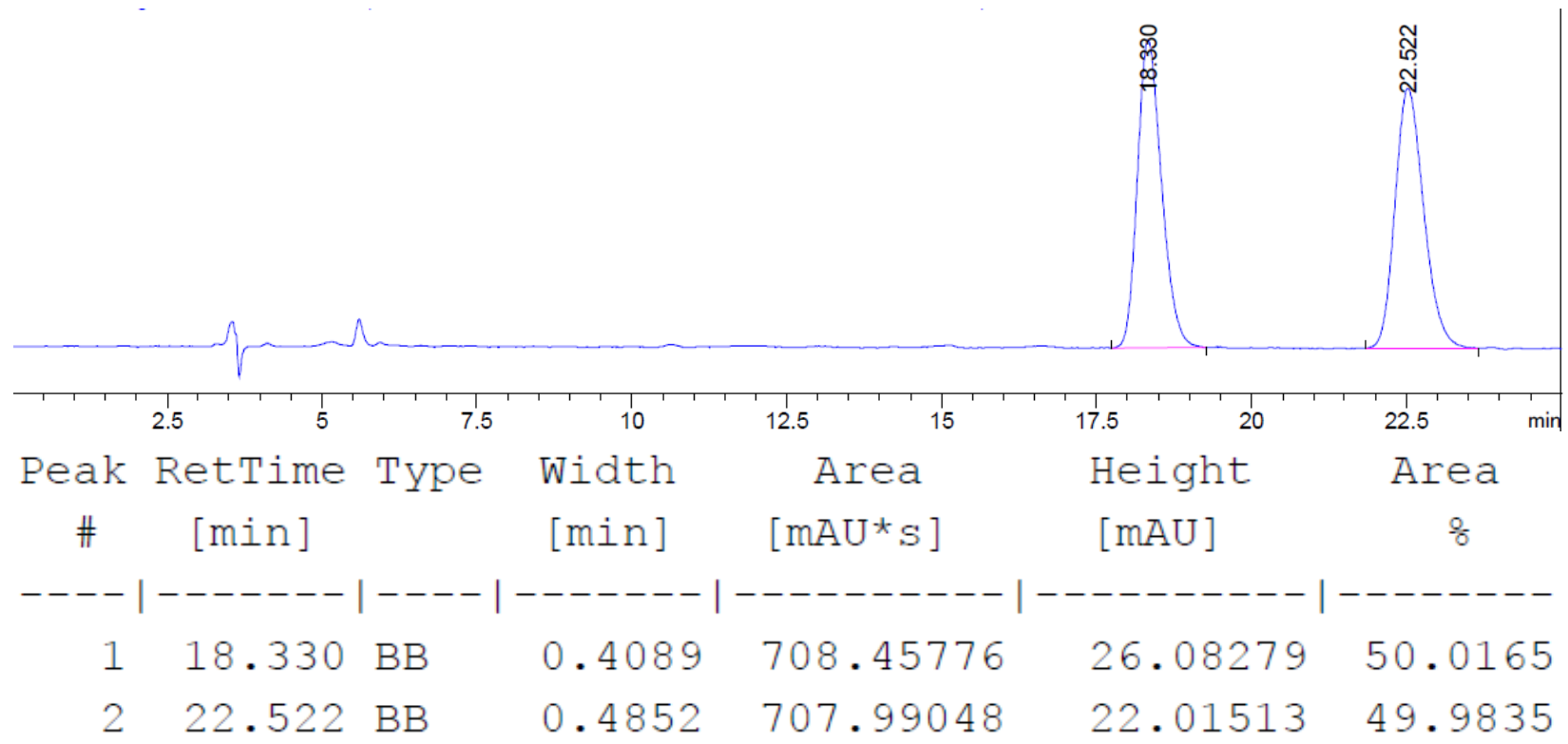

$(-)-(S)-3-($ morpholine-4-carbonyl)-3,4-dihydroisoquinolin-1(2H)-one (3ai)<smiles>O=C1N[C@H](C(=O)N2CCOCC2)Cc2ccccc21</smiles>

With catalyst Co7: General Procedure C. Yield: $44 \%$ (23.0 mg, $88.0 \mu \mathrm{mol})$, white solid. ${ }^{1} \mathrm{H}$ NMR $\left(400 \mathrm{MHz}, \mathrm{CDCl}_{3}\right) \delta=8.05$ (dd, $\left.J=7.7,1.4 \mathrm{~Hz}, 1 \mathrm{H}\right), 7.46$ (td, $J$ $=7.5,1.5 \mathrm{~Hz}, 1 \mathrm{H}), 7.37(\mathrm{t}, J=7.5 \mathrm{~Hz}, 1 \mathrm{H}), 7.21(\mathrm{~d}, J=7.5 \mathrm{~Hz}, 1 \mathrm{H}), 6.75(\mathrm{~s}, 1 \mathrm{H})$, 4.67 (dd, $J=11.4,4.1 \mathrm{~Hz}, 1 \mathrm{H}), 3.79-3.66(\mathrm{~m}, 5 \mathrm{H}), 3.63-3.47(\mathrm{~m}, 3 \mathrm{H}), 3.19$ (dd, $J=15.5,11.4 \mathrm{~Hz}, 1 \mathrm{H}), 3.03(\mathrm{dd}, J=15.5,4.1 \mathrm{~Hz}, 1 \mathrm{H}) \mathrm{ppm} ;{ }^{13} \mathbf{C}\left\{{ }^{1} \mathrm{H}\right\}$ NMR $\left(101 \mathrm{MHz}, \mathrm{CDCl}_{3}\right) \delta=168.2,165.3,136.2,132.6,129.0,128.3,127.9,127.3$, 66.9, 66.6, 51.8, 46.1 , 42.9, 32.2 ppm; IR (ATR): 3276, 2962, 2921, 2854, 1665, 1606, 1463, 1434, 1270, 1244, 1113, 1038, 748, $568 \mathrm{~cm}^{-1}$; HRMS (ESI): calcd. for $\left[\mathrm{C}_{14} \mathrm{H}_{17} \mathrm{~N}_{2} \mathrm{O}_{3}\right]^{+},\left[\mathrm{M}_{+} \mathrm{H}^{+}\right]^{+}: 261.1234$; found: $261.1231 ; \boldsymbol{R}_{\mathrm{f}}: 0.04$ (hexane/EtOAc $\left.=1 / 1\right)$; $[\alpha]_{\mathrm{D}}^{20}:-154.3\left(c=0.50, \mathrm{CHCl}_{3}\right)$; Chiral HPLC: Chiralpak IA, $4.6 \times 250 \mathrm{~mm}$; 30\% i-PrOH/hexane, $1.0 \mathrm{~mL} / \mathrm{min}, 254 \mathrm{~nm}$; tr (major) = $21.4 \mathrm{~min}$, tr (minor) $=27.6 \mathrm{~min}, 99.5 / 0.5 \mathrm{er}$. 

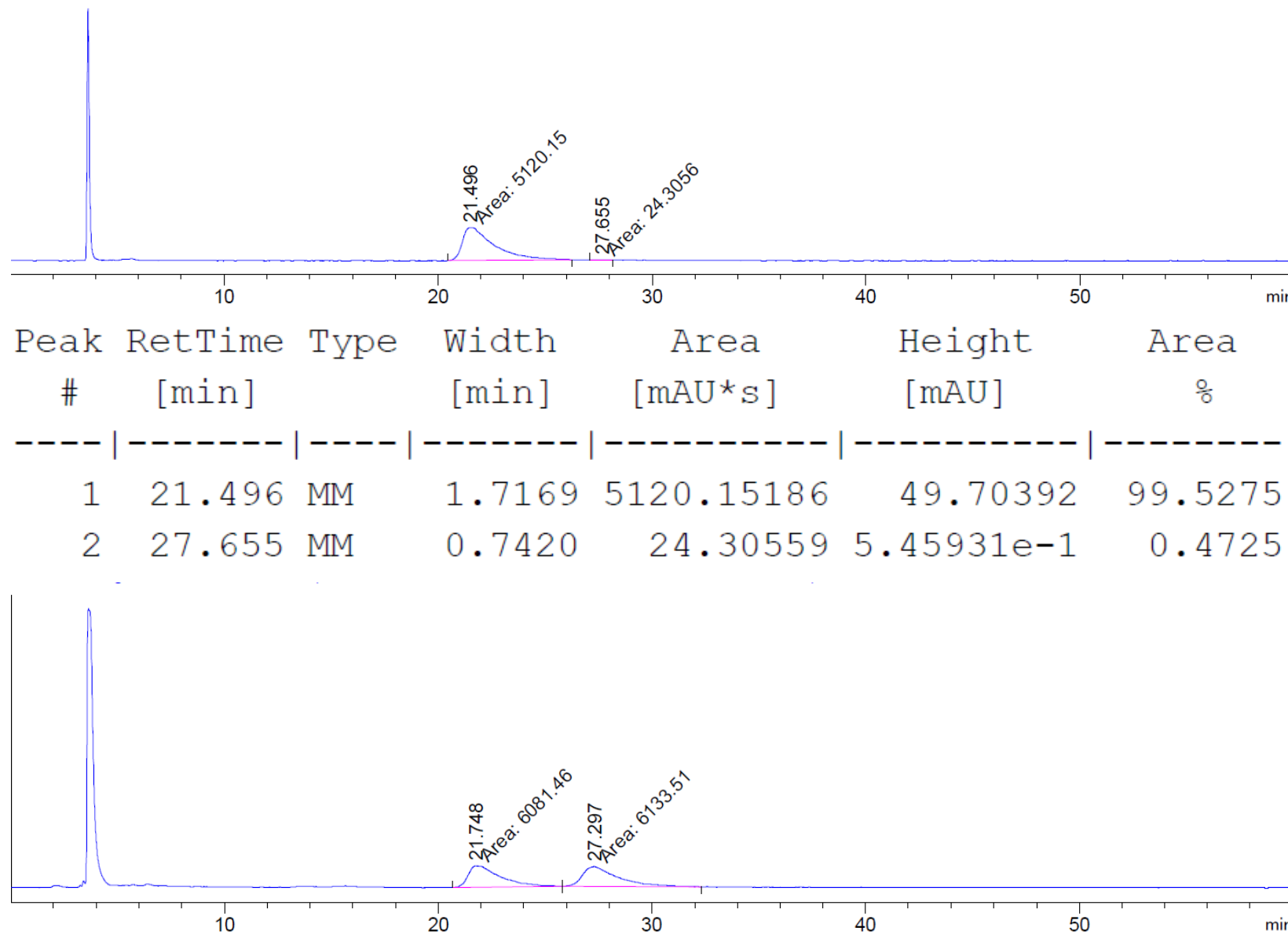

Peak RetTime Type Width

Area

40

$\# \quad[\mathrm{~min}] \quad[\mathrm{min}] \quad\left[\mathrm{mAU}^{*} \mathrm{~s}\right] \quad[\mathrm{mAU}] \quad \frac{\circ}{\circ}$

----|-------|----|-------|----------|----------|--------

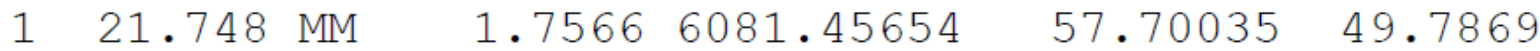

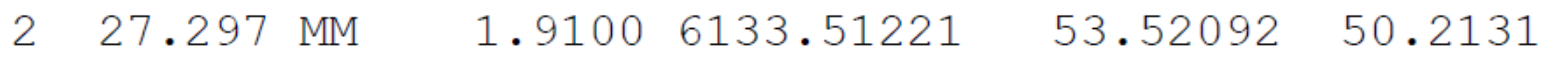

With catalyst Co6: General Procedure C. The amount of alkene was increased to $85.0 \mathrm{mg}$, $0.60 \mathrm{mmol}$, 3.0 equiv. Yield: $74 \%$ (38.5 mg, $148 \mu \mathrm{mol})$, white solid. ; $[\alpha]_{D}^{20}:-151.0\left(c=1.00, \mathrm{CHCl}_{3}\right)$. Chiral HPLC: Chiralpak IF, $4.6 \times 250 \mathrm{~mm} ; 50 \% \mathrm{i}-\mathrm{PrOH} /$ hexane, $1.0 \mathrm{~mL} / \mathrm{min}, 254 \mathrm{~nm}$; $\operatorname{tr}$ (major) = $36.9 \mathrm{~min}, \operatorname{tr}($ minor $)=52.6 \mathrm{~min}, 93.2 / 6.8 \mathrm{er}$. 

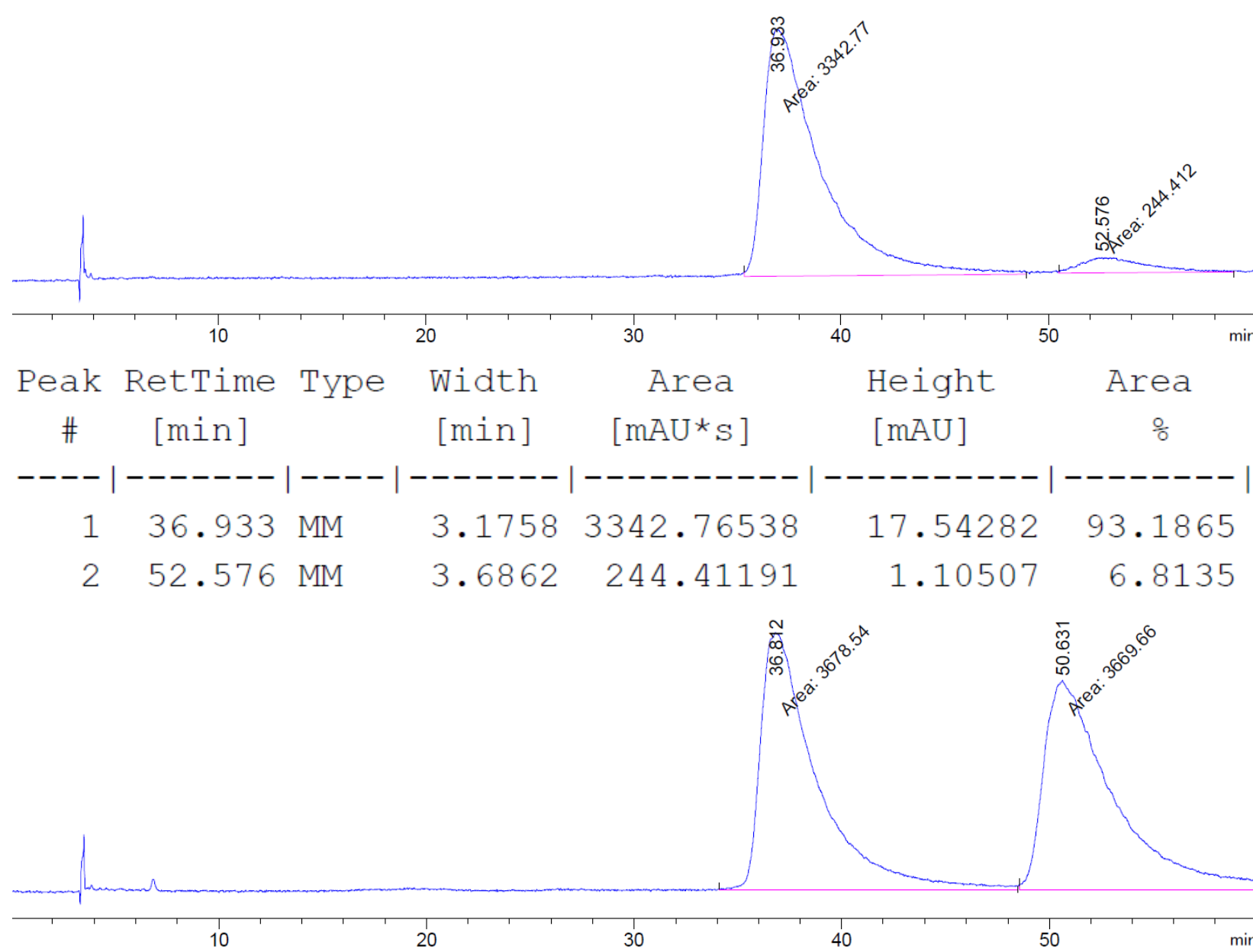

\begin{tabular}{|c|c|c|c|c|c|c|}
\hline $\begin{array}{c}\text { Peak } \\
\#\end{array}$ & $\begin{array}{c}\text { RetTime } \\
\text { [min] }\end{array}$ & Type & $\begin{array}{l}\text { Width } \\
\text { [min] }\end{array}$ & $\begin{array}{c}\text { Area } \\
{\left[\mathrm{mAU}^{*} \mathrm{~s}\right]}\end{array}$ & $\begin{array}{l}\text { Height } \\
{[\mathrm{mAU}]}\end{array}$ & $\begin{array}{c}\text { Area } \\
\text { 응 }\end{array}$ \\
\hline & & & & ------ & & \\
\hline 1 & 6.812 & $\mathrm{MM}$ & 3.1323 & 3678.53906 & 19.57321 & \\
\hline 0 & 50.631 & $\mathrm{MM}$ & 3.8431 & 3669.66064 & 15.91445 & \\
\hline
\end{tabular}

(+)-(R)-2-(1-oxo-1,2,3,4-tetrahydroisoquinolin-3-yl)isoindoline-1,3-dione (3aj)<smiles>O=C1N[C@H](N2C(=O)c3ccccc3C2=O)Cc2ccccc21</smiles>

General procedure A. $N$-Vinyl phthalimide was added to the reaction mixture as a solid. Yield: $68 \%(40.0 \mathrm{mg}, 137 \mu \mathrm{mol})$, white solid. ${ }^{1} \mathbf{H}$ NMR $\left(400 \mathrm{MHz}_{\mathrm{CDCl}}\right) \mathrm{CD}=8.06(\mathrm{~d}, J=7.7,0.9 \mathrm{~Hz}, 1 \mathrm{H}), 7.79-7.73(\mathrm{~m}, 2 \mathrm{H})$, 7.71-7.65 (m, 2H), 7.50 (td, $J=7.5,1.3 \mathrm{~Hz}, 1 \mathrm{H}), 7.36$ (t, $J=7.5 \mathrm{~Hz}, 1 \mathrm{H}$ ), 7.24 (d, $J=7.5 \mathrm{~Hz}, 1 \mathrm{H}$ ), 6.91 (s, 1H), 6.03-5.97 (m, 1H), 3.60 (dd, J=16.6, $4.5 \mathrm{~Hz}, 1 \mathrm{H}), 3.51$ (dd, $J=16.6,6.5 \mathrm{~Hz}, 1 \mathrm{H}) \mathrm{ppm} ;{ }^{13} \mathrm{C}\left\{{ }^{1} \mathrm{H}\right\} \mathrm{NMR} \delta=\left(101 \mathrm{MHz}, \mathrm{CDCl}_{3}\right)$ 167.4, 165.6, 136.5, 134.5, 132.7, 131.6, 127.9, 127.8, 127.4, 127.3, 123.7, 58.2, 31.4 ppm; IR (ATR) 3317, 3061, 2959, 1776, 1713, 1672, 1468, 1387, 1364, 1323, 1284, 1111, 742, $718 \mathrm{~cm}^{-1}$; HRMS (ESI/QTOF) calcd. for $\left[\mathrm{C}_{17} \mathrm{H}_{12} \mathrm{~N}_{2} \mathrm{NaO}_{3}\right]^{+},[\mathrm{M}+\mathrm{Na}]^{+}: 315.0740 ;$ found: $315.0745 ; \boldsymbol{R}_{\mathrm{f}}: 0.45$ 
(hexane/EtOAc $=1 / 2) ;[\alpha]_{D}^{20}:-151.7\left(c=1.00, \mathrm{CHCl}_{3}\right)$; Chiral HPLC: Chiralpak IB, $4.6 \times 250 \mathrm{~mm}$; $40 \% \mathrm{i}-\mathrm{PrOH} /$ hexane, $1.0 \mathrm{~mL} / \mathrm{min}, 254 \mathrm{~nm}$; $\operatorname{tr}$ (major) $=12.0 \mathrm{~min}, \operatorname{tr}(\operatorname{minor})=14.5 \mathrm{~min}, 98.8 / 1.2 \mathrm{er}$.
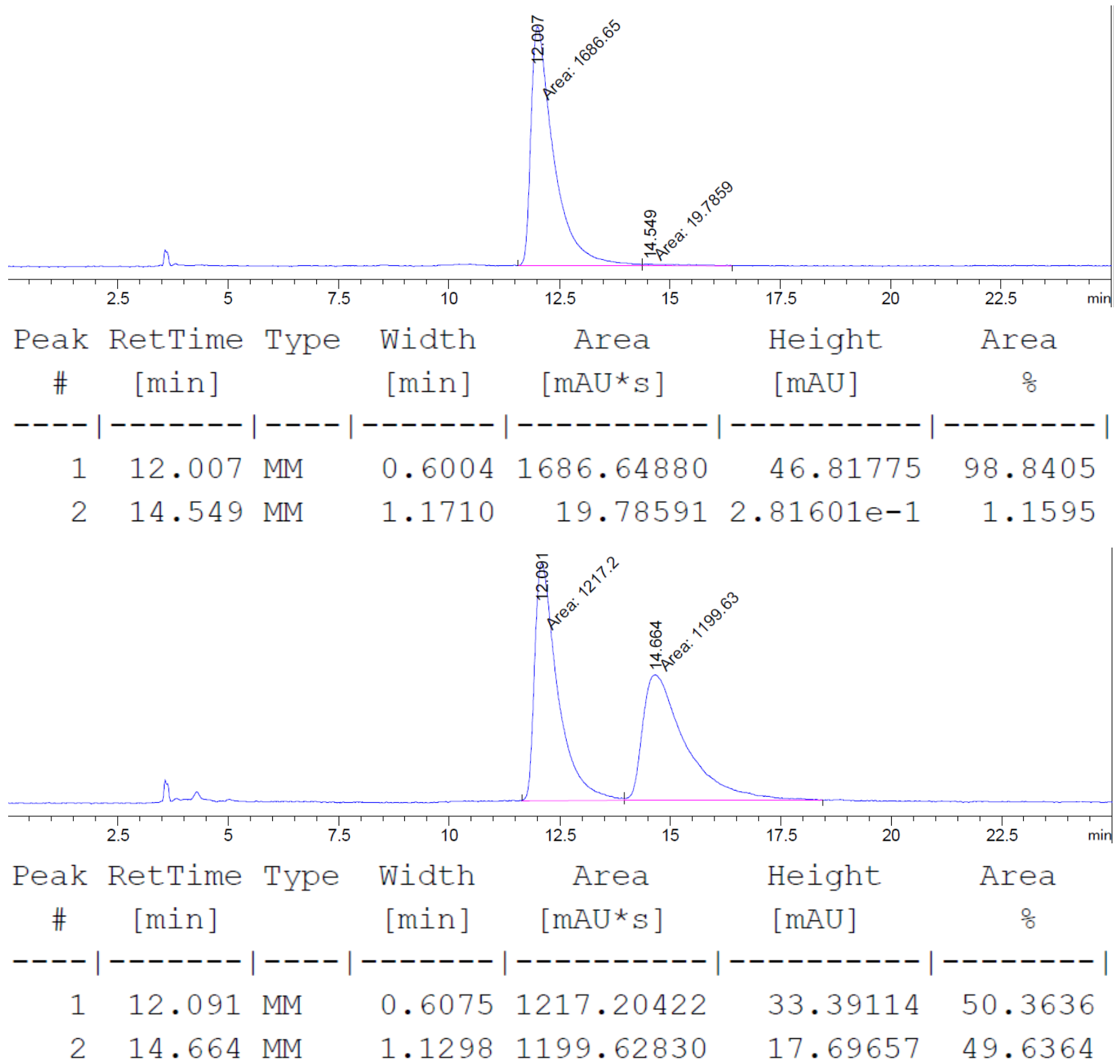

$(-)-(R)$-3-butyl-3,4-dihydroisoquinolin-1(2H)-one (3ak)

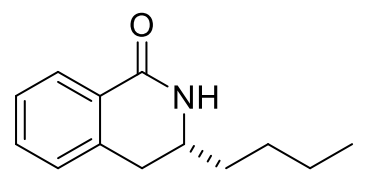

General Procedure A. Yield: 86\% (35.0 mg, $172 \mu \mathrm{mol}$ ), off-white solid. The spectra match with the literature values ${ }^{10} .{ }^{1} \mathrm{H}$ NMR $\left(400 \mathrm{MHz}, \mathrm{CDCl}_{3}\right) \delta=$ 8.05 (d, $J=7.6,1 \mathrm{H}$ ), 7.44 (t, $J=7.6,1 \mathrm{H}$ ), 7.33 (d, $J=7.6,1 \mathrm{H}$ ), 7.20 (d, $J=$ 7.5, 1H), $6.09(\mathrm{~s}, 1 \mathrm{H}), 3.73-3.63(\mathrm{~m}, 1 \mathrm{H}), 2.97$ (dd, $J=15.6,4.3,1 \mathrm{H}), 2.81$ (dd, $J=15.6,10.4$, $1 \mathrm{H}), 1.60(\mathrm{t}, J=7.2,7.2,2 \mathrm{H}), 1.37(\mathrm{~s}, 4 \mathrm{H}), 0.92(\mathrm{t}, J=6.7,3 \mathrm{H}) \mathrm{ppm} ;{ }^{13} \mathrm{C}\left\{{ }^{1} \mathrm{H}\right\} \mathbf{N M R}(101 \mathrm{MHz}$, $\left.\mathrm{CDCl}_{3}\right) \delta=166.4,138.3,132.4,128.7,128.1,127.6,127.2,51.5,35.2,34.5,27.7,22.6,14.1$ ppm; 
$\boldsymbol{R}_{\mathrm{f}}: 0.33$ (hexane/EtOAc = 2/1); $[\alpha]_{\mathrm{D}}^{20}:-33.5\left(c=1.00, \mathrm{CHCl}_{3}\right)$; Chiral HPLC: Chiralpak IB, $4.6 \mathrm{x}$ $250 \mathrm{~mm} ; 10 \% \mathrm{i}-\mathrm{PrOH} /$ hexane, $1.0 \mathrm{~mL} / \mathrm{min}, 254 \mathrm{~nm}$; tr (minor) = $7.7 \mathrm{~min}$, tr (major) = $8.2 \mathrm{~min}$, 95.5/4.5 er.
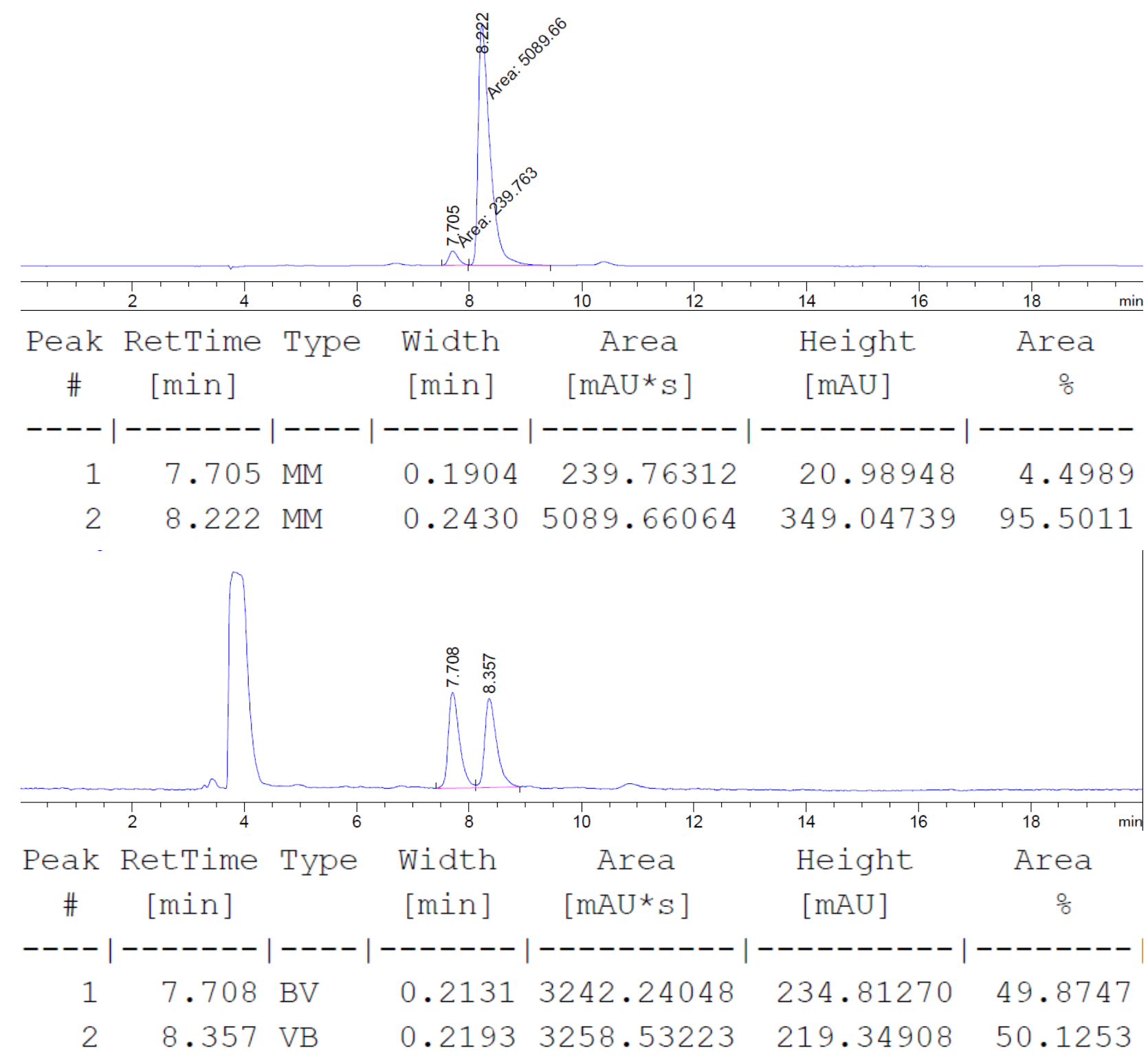

(-)- $(R)$-3-hexyl-3,4-dihydroisoquinolin-1(2H)-one (3al)

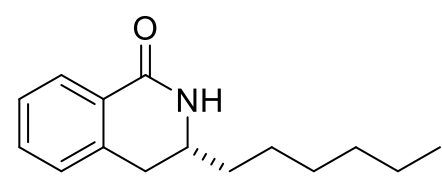

General Procedure A. Yield: 74\% (34.3 mg, $148 \mu \mathrm{mol})$, off-white solid. The spectra match with the literature values. ${ }^{8}{ }^{1} \mathbf{H}$ NMR $(400 \mathrm{MHz}$, $\left.\mathrm{CDCl}_{3}\right) \delta=8.04(\mathrm{dd}, J=7.5,0.8 \mathrm{~Hz}, 1 \mathrm{H}), 7.42(\mathrm{dt}, J=7.5,1.3 \mathrm{~Hz}, 1 \mathrm{H})$, $7.32(\mathrm{t}, J=7.5 \mathrm{~Hz}, 1 \mathrm{H}), 7.18(\mathrm{~d}, J=7.5 \mathrm{~Hz}, 1 \mathrm{H}), 6.53(\mathrm{~s}, 1 \mathrm{H}), 3.72-3.63(\mathrm{~m}, 1 \mathrm{H}), 2.96(\mathrm{dd}, J=$ 15.6, 4.4 Hz, 1H), 2.79 (dd, $J=15.6,10.3 \mathrm{~Hz}, 1 \mathrm{H}), 1.68-1.52(\mathrm{~m}, 2 \mathrm{H}), 1.47-1.22(\mathrm{~m}, 8 \mathrm{H}), 0.87$ $(\mathrm{t}, J=6.7 \mathrm{~Hz}, 3 \mathrm{H})$ ppm; $\boldsymbol{R}_{\mathrm{f}}: 0.40$ (hexane/EtOAc = 1/1); [a] $]_{\mathrm{D}}^{20}:-49.0\left(c=1.00, \mathrm{CHCl}_{3}\right) ;$ Chiral HPLC: 
Chiralpak IB, 4.6 x $250 \mathrm{~mm} ; 10 \%$ i-PrOH/hexane, $1.0 \mathrm{~mL} / \mathrm{min}, 254 \mathrm{~nm}$; tr (minor) = $7.2 \mathrm{~min}$, tr (major) $=7.9 \mathrm{~min}, 94.7 / 5.3 \mathrm{er}$.
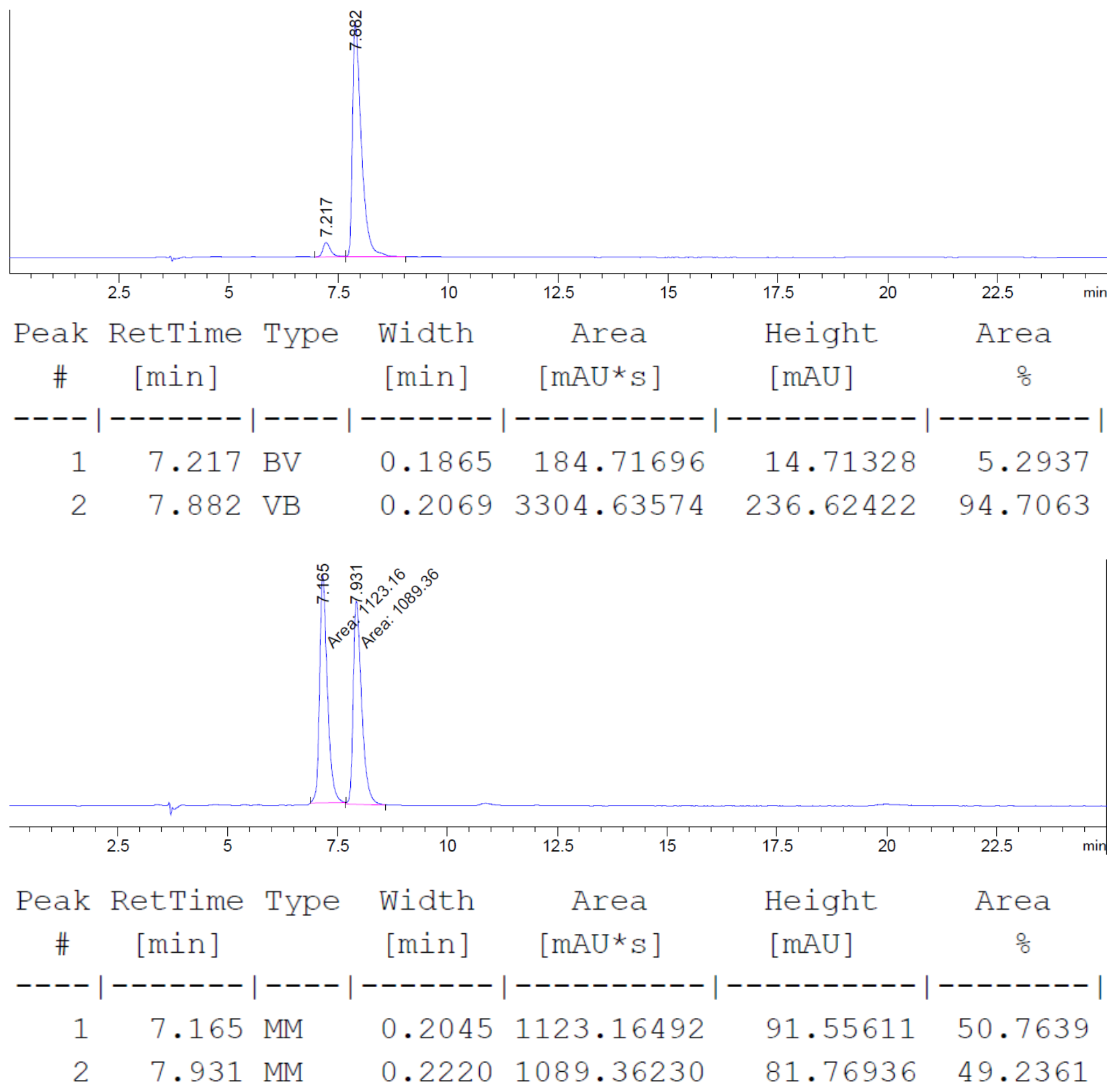

\section{(-)-(S)-3-(2-(benzyloxy)ethyl)-3,4-dihydroisoquinolin-1(2H)-one (3am)}<smiles>O=C1N[C@H](CCOc2ccccc2)Cc2ccccc21</smiles>

General Procedure C. Yield: $78 \%(44.0 \mathrm{mg}, 156 \mu \mathrm{mol})$, off-white solid.

${ }^{1} \mathrm{H}$ NMR $\left(400 \mathrm{MHz}, \mathrm{CDCl}_{3}\right) \delta=8.06(\mathrm{~d}, J=7.6,1 \mathrm{H}), 7.43(\mathrm{t}, J=7.4,1 \mathrm{H}), 7.40$ - $7.28(\mathrm{~m}, 6 \mathrm{H}), 7.17(\mathrm{~d}, J=7.4,1 \mathrm{H}), 6.68(\mathrm{~s}, 1 \mathrm{H}), 4.55(\mathrm{~s}, 2 \mathrm{H}), 3.95-3.86$ (m, 1H), $3.71-3.56(\mathrm{~m}, 2 \mathrm{H}), 2.98-2.81(\mathrm{~m}, 2 \mathrm{H}), 2.03-1.89(\mathrm{~m}, 1 \mathrm{H}), 1.87-1.76(\mathrm{~m}, 1 \mathrm{H}) \mathrm{ppm}$; ${ }^{13} \mathbf{C}\left\{{ }^{1} \mathrm{H}\right\}$ NMR $\left(101 \mathrm{MHz}, \mathrm{CDCl}_{3}\right) \delta=166.5$ (see HMBC), 138.1, 137.9, 132.3, 128.7, 128.1, 128.0, 127.9, 127.5, 127.2, 73.5, 68.0, 50.9, 35.2, 34.9 ppm; IR (ATR): 3201, 3064, 2918, 2861, 1664, 
1605, 1578, 1465, 1398, 1341, 1092, 744, $698 \mathrm{~cm}^{-1} ; \boldsymbol{R}_{\mathrm{f}}: 0.12$ (hexane/EtOAc = 2/1); [a] $]_{\mathrm{D}}^{20}:-35.0$ ( $\left.c=1.00, \mathrm{CHCl}_{3}\right)$; HRMS (ESI): calcd. for $\left[\mathrm{C}_{18} \mathrm{H}_{20} \mathrm{NO}_{2}\right]^{+},\left[\mathrm{M}+\mathrm{H}^{+}\right]^{+}:$282.1489; found: 282.1490; Chiral HPLC: Chiralpak IA, 4.6 x $250 \mathrm{~mm} ; 20 \%$ i-PrOH/hexane, $1.0 \mathrm{~mL} / \mathrm{min}, 254 \mathrm{~nm}$; tr (major) = $8.5 \mathrm{~min}, \operatorname{tr}$ (minor) $=9.5 \mathrm{~min}, 92.8 / 7.2 \mathrm{er}$.
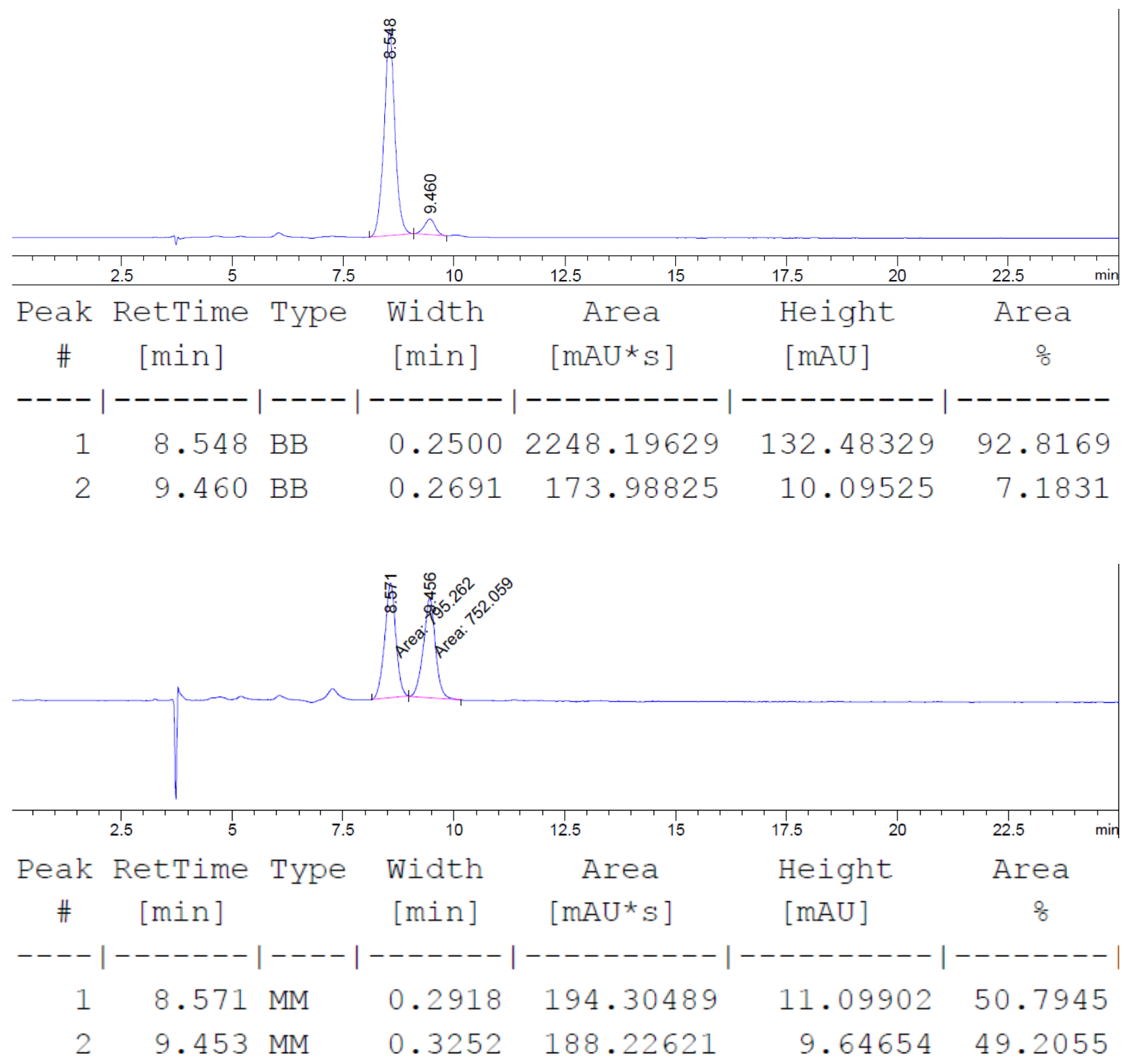

\section{(-)-(S)-3-(2-hydroxyethyl)-3,4-dihydroisoquinolin-1(2H)-one (3an)}

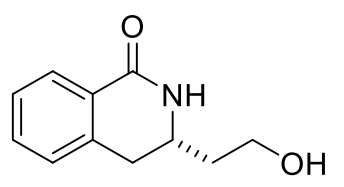

General Procedure C. Increased amount of CsOPiv $(84.0 \mathrm{mg}, 360 \mu \mathrm{mol}$, 1.80 equiv.) used. Yield: 49\% (18.6 mg, $97.0 \mu \mathrm{mol})$, white solid. ${ }^{1} \mathbf{H}$ NMR $\left(400 \mathrm{MHz}, \mathrm{CDCl}_{3}\right) \delta=8.02(\mathrm{dd}, J=7.5,0.9 \mathrm{~Hz}, 1 \mathrm{H}), 7.59(\mathrm{~s}, 1 \mathrm{H}), 7.43(\mathrm{td}, J$ 
$=7.5 \mathrm{~Hz}, 1.3 \mathrm{~Hz}, 1 \mathrm{H}), 7.33$ (t, $J=7.5,1 \mathrm{H}), 7.18(\mathrm{~d}, J=7.5 \mathrm{~Hz}, 1 \mathrm{H}), 4.04-3.89(\mathrm{~m}, 2 \mathrm{H}), 3.89-$ $3.81(\mathrm{~m}, 1 \mathrm{H}), 3.74(\mathrm{~s}, 1 \mathrm{H}), 2.93(\mathrm{dd}, J=15.6,5.1 \mathrm{~Hz}, 1 \mathrm{H}), 2.87$ (dd, $J=15.6,10.4 \mathrm{~Hz}, 1 \mathrm{H}), 1.98$ $-1.87(\mathrm{~m}, 1 \mathrm{H}), 1.83-1.74(\mathrm{~m}, 1 \mathrm{H}) \mathrm{ppm} ;{ }^{13} \mathrm{C}\left\{{ }^{1} \mathrm{H}\right\} \mathbf{N M R}\left(101 \mathrm{MHz}, \mathrm{CDCl}_{3}\right) \delta=166.7,138.3,132.4$, 128.5, 128.0, 127.5, 127.2, 60.9, 51.4, 37.0, 34.9 ppm; IR (ATR): 3305, 2925, 2882, 1652, 1604 , $1465,1400,1341,1064,1034,745 \mathrm{~cm}^{-1} ; \boldsymbol{R}_{\mathrm{f}}: 0.60(\mathrm{DCM} / \mathrm{MeOH}=10 / 1) ;[\alpha]_{\mathrm{D}}^{20}:-106.5(c=1.00$, $\mathrm{CHCl}_{3}$ ); HRMS (ESI/QTOF): calcd. for $\left[\mathrm{C}_{11} \mathrm{H}_{14} \mathrm{NO}_{2}\right]^{+},\left[\mathrm{M}+\mathrm{H}^{+}\right]^{+}:$192.1019; found: 192.1012; Chiral HPLC: Chiralpak IC, $4.6 \times 250 \mathrm{~mm} ; 20 \% \mathrm{i}-\mathrm{PrOH} /$ hexane, $1.0 \mathrm{~mL} / \mathrm{min}, 254 \mathrm{~nm}$; tr (minor) = 36.6 $\min , \operatorname{tr}$ (major) $=42.0 \mathrm{~min}, 98.1 / 1.9 \mathrm{er}$.

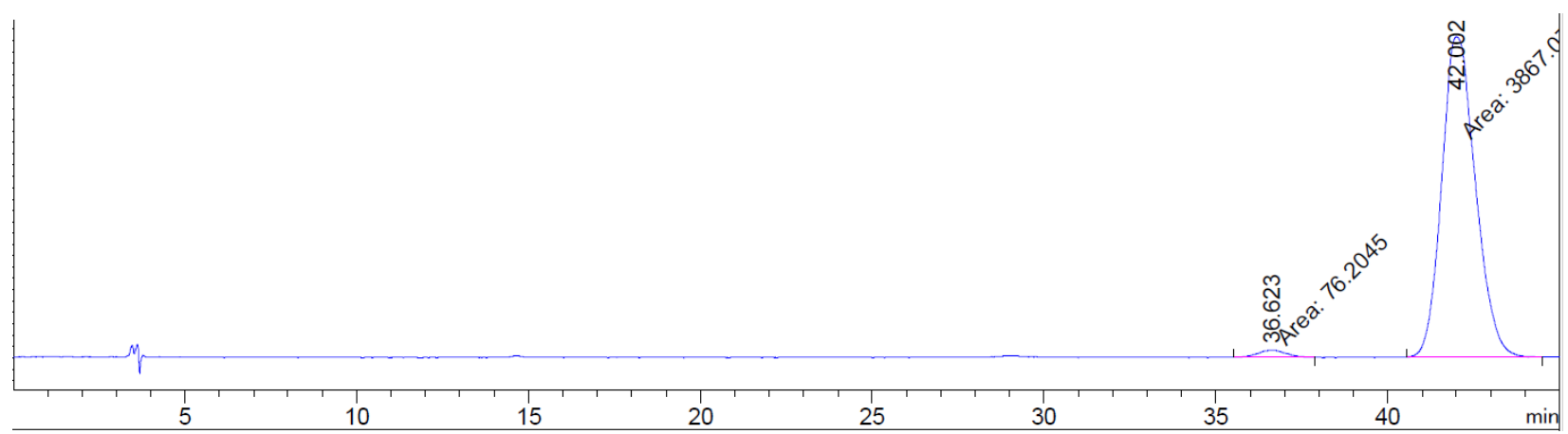

Peak RetTime Type Width Area Height Area

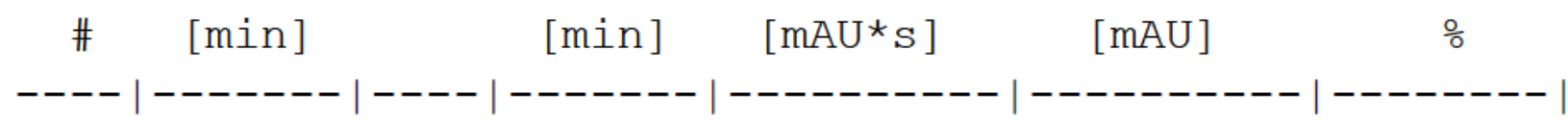
$136.623 \mathrm{MM}$
$0.9457 \quad 76.20451$
1.34305
1.9325

$242.002 \mathrm{MM}$

1.13963867 .07324

$56.55775 \quad 98.0675$

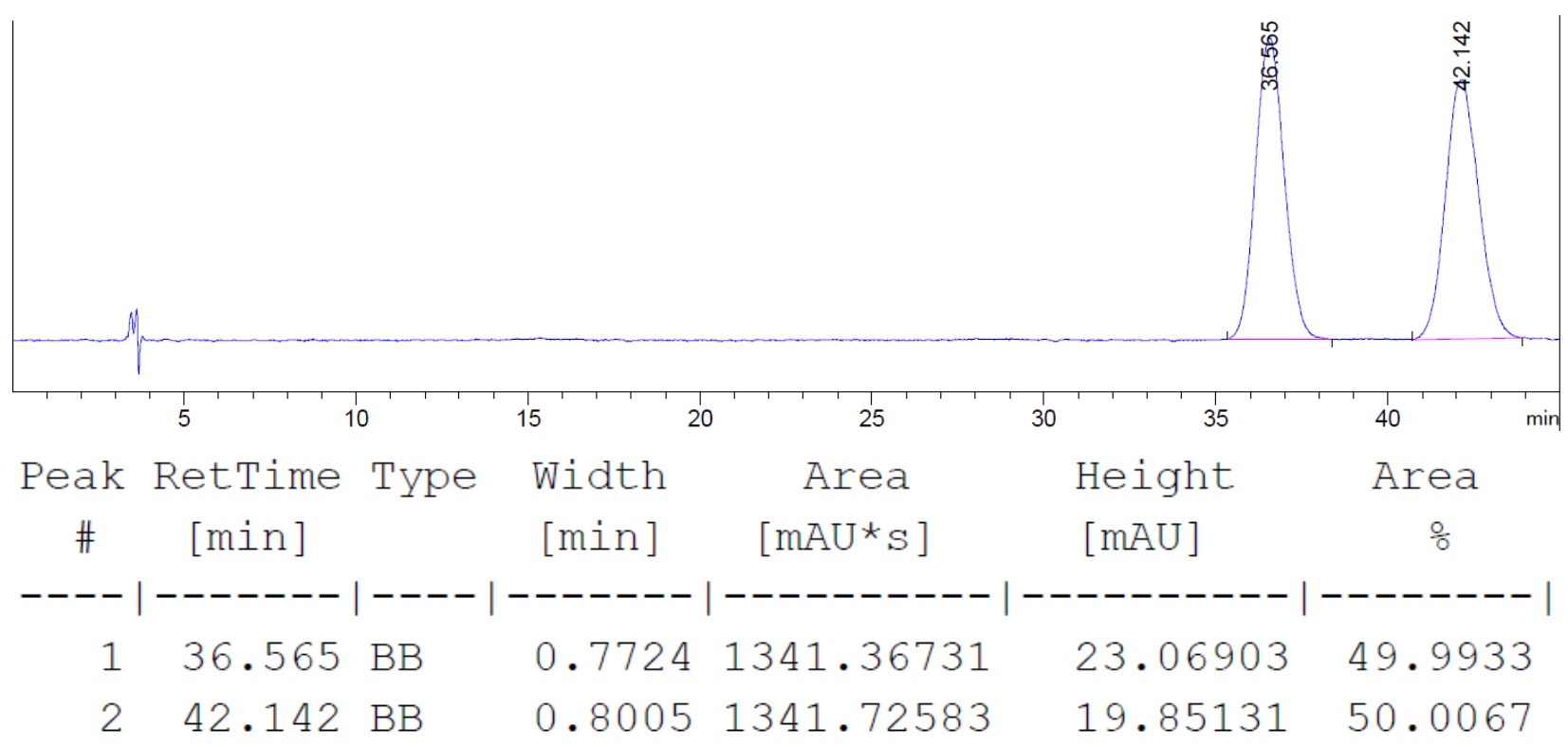




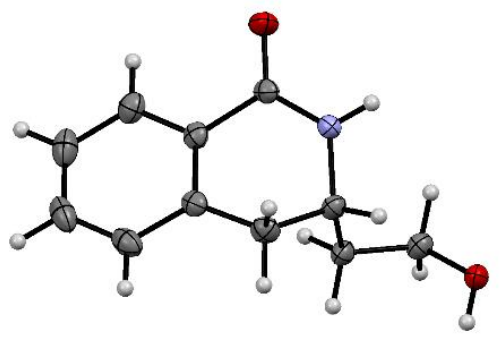

X-Ray structure of racemic 3an (CCDC No: 1898854).

\section{(-)-(R)-4-(2-hydroxyethyl)-3,4-dihydroisoquinolin-1(2H)-one (3an')}<smiles>O=C1NC[C@H](CCO)c2ccccc21</smiles>

General procedure C. Increased amount of CsOPiv $(84.0 \mathrm{mg}, 360 \mu \mathrm{mol}, 1.80$ equiv.) used. Yield: $21 \%(8.0 \mathrm{mg}, 42.0 \mu \mathrm{mol})$, white solid. The spectra match with the literature values. ${ }^{11}$ Absolute configuration determined by comparison with the reported $[\alpha]_{D}^{20}$ value. ${ }^{12}{ }^{1} \mathrm{H}$ NMR $\left(400 \mathrm{MHz}, \mathrm{CDCl}_{3}\right) \delta=8.06(\mathrm{~d}, J=7.6 \mathrm{~Hz}, 1 \mathrm{H}), 7.46$ (td, $J=7.6 \mathrm{~Hz}, 1.3 \mathrm{~Hz}, 1 \mathrm{H}), 7.36$ (td, $J=7.6,1.0 \mathrm{~Hz}, 1 \mathrm{H}), 7.26(\mathrm{~d}, J=7.6 \mathrm{~Hz}, 1 \mathrm{H})$, $6.56-6.43(\mathrm{~m}, 1 \mathrm{H}), 3.80-3.69(\mathrm{~m}, 2 \mathrm{H}), 3.68-3.61(\mathrm{~m}, 1 \mathrm{H}), 3.47-3.40(\mathrm{~m}, 1 \mathrm{H}), 3.17-3.09$ $(\mathrm{m}, 1 \mathrm{H}), 2.00-1.86(\mathrm{~m}, 3 \mathrm{H}) \mathrm{ppm} ; \boldsymbol{R}_{\mathrm{f}}: 0.50(\mathrm{DCM} / \mathrm{MeOH}=10 / 1) ;[\alpha]_{\mathrm{D}}^{20}:-93.3\left(c=0.50, \mathrm{CHCl}_{3}\right)$; Chiral HPLC: Chiralpak IG, $4.6 \times 250 \mathrm{~mm} ; 20 \% \mathrm{i}-\mathrm{PrOH} /$ hexane, $1.0 \mathrm{~mL} / \mathrm{min}, 254 \mathrm{~nm}$; $\operatorname{tr}$ (major) = $13.2 \mathrm{~min}, \operatorname{tr}(\mathrm{minor})=14.8 \mathrm{~min}, 78.2 / 21.8 \mathrm{er}$.

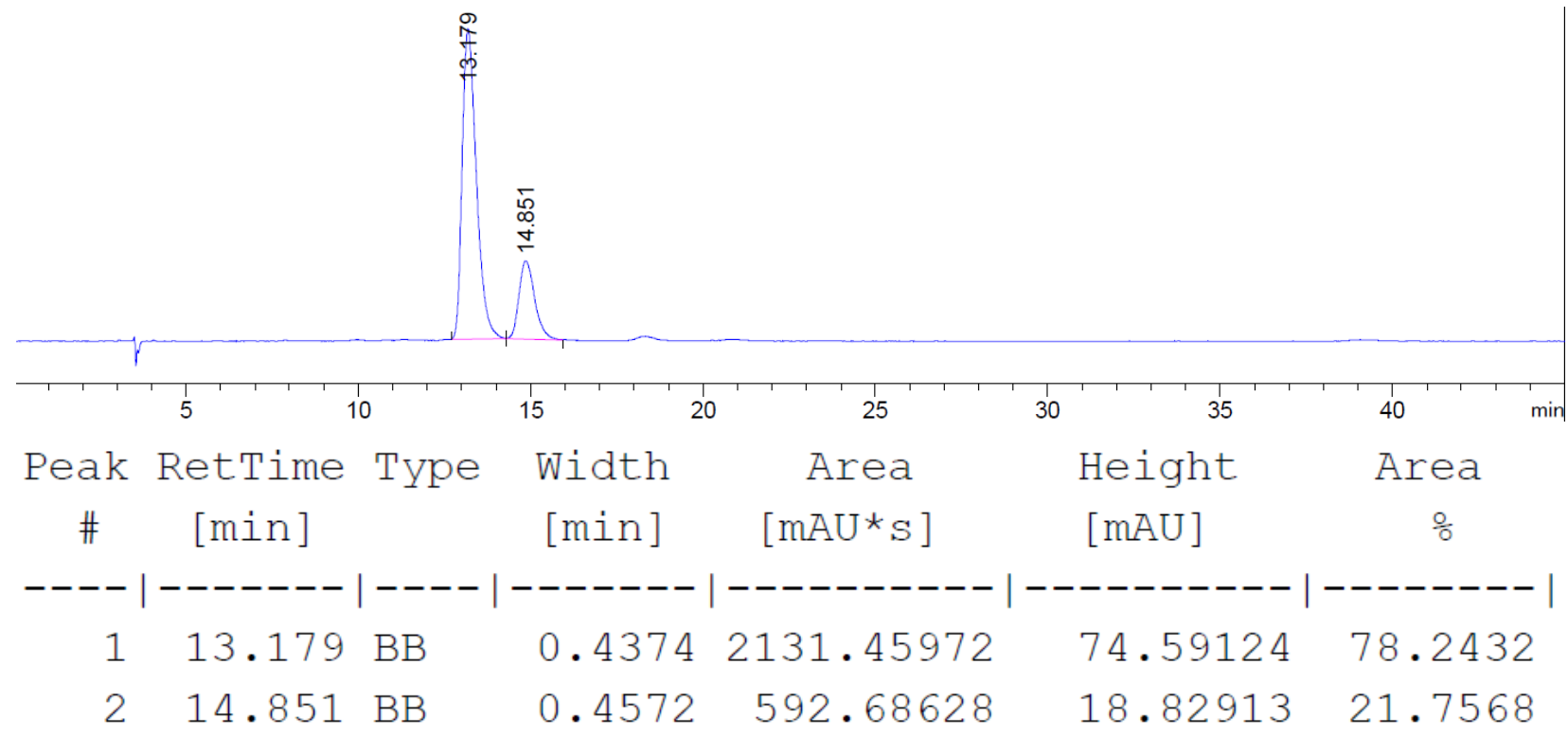




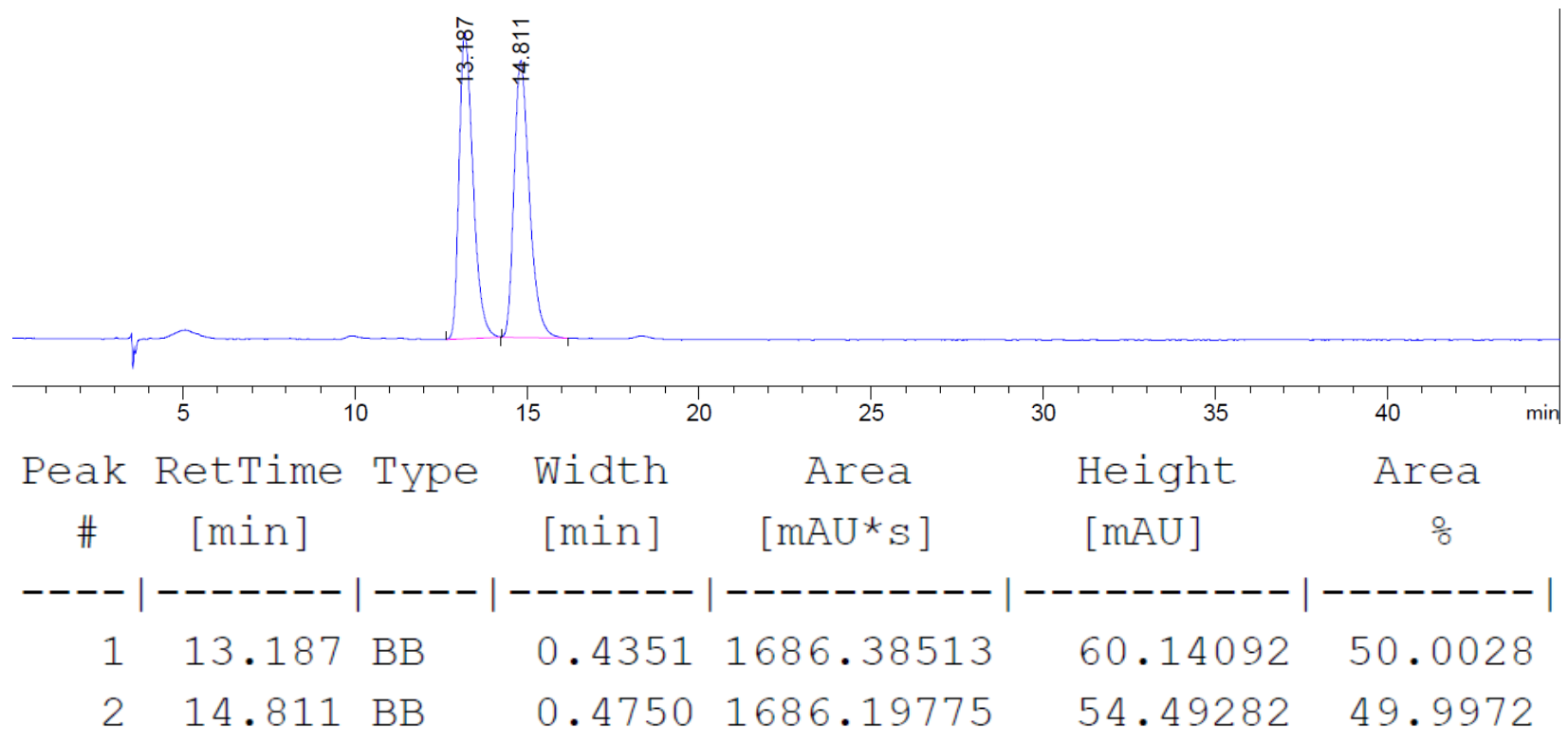

\section{(+)-(S)-3-Acetoxymethyl-3,4-dihydroisoquinolin-1(2H)-one (3ao)}<smiles>CC(=O)OC[C@H]1Cc2ccccc2C(=O)N1</smiles>

General Procedure A. Yield: 77\% (33.7 mg, $154 \mu \mathrm{mol})$, white solid. The spectra match with the literature values. ${ }^{13}{ }^{1} \mathrm{H}$ NMR $\left(400 \mathrm{MHz}, \mathrm{CDCl}_{3}\right) \delta=$ 8.06 (dd, $J=7.7,1.0 \mathrm{~Hz}, 1 \mathrm{H}$ ), 7.45 (td, $J=7.5 \mathrm{~Hz}, J=1.3 \mathrm{~Hz}, 1 \mathrm{H}), 7.35$ (t, $J=7.5 \mathrm{~Hz}, 1 \mathrm{H}), 7.21(\mathrm{~d}, J=7.5 \mathrm{~Hz}, 1 \mathrm{H}), 6.62(\mathrm{~s}, 1 \mathrm{H}), 4.25$ (dd, $J=11.0$, $4.3 \mathrm{~Hz}, 1 \mathrm{H}), 4.08(\mathrm{dd}, J=11.0,7.9 \mathrm{~Hz}, 1 \mathrm{H}), 4.04-3.96(\mathrm{~m}, 1 \mathrm{H}), 3.04(\mathrm{dd}, J=15.7,4.8 \mathrm{~Hz}, 1 \mathrm{H})$, 2.90 (dd, $J=15.7,9.0 \mathrm{~Hz}, 1 \mathrm{H}), 2.07(\mathrm{~s}, 3 \mathrm{H}) \mathrm{ppm} ; \boldsymbol{R}_{\mathrm{f}}: 0.40(\mathrm{DCM} / \mathrm{EtOAc}=2 / 1) ;[\alpha]_{\mathrm{D}}^{20}:+3.63(c=$ 1.00, $\mathrm{CHCl}_{3}$ ); Chiral HPLC: Chiralpak IA, 4.6 x $250 \mathrm{~mm} ; 30 \%$ i-PrOH/hexane, $1.0 \mathrm{~mL} / \mathrm{min}, 254$ $\mathrm{nm} ; \operatorname{tr}($ major $)=6.9 \mathrm{~min}, \operatorname{tr}($ minor $)=8.3 \mathrm{~min}, 95.8 / 4.2 \mathrm{er}$. 

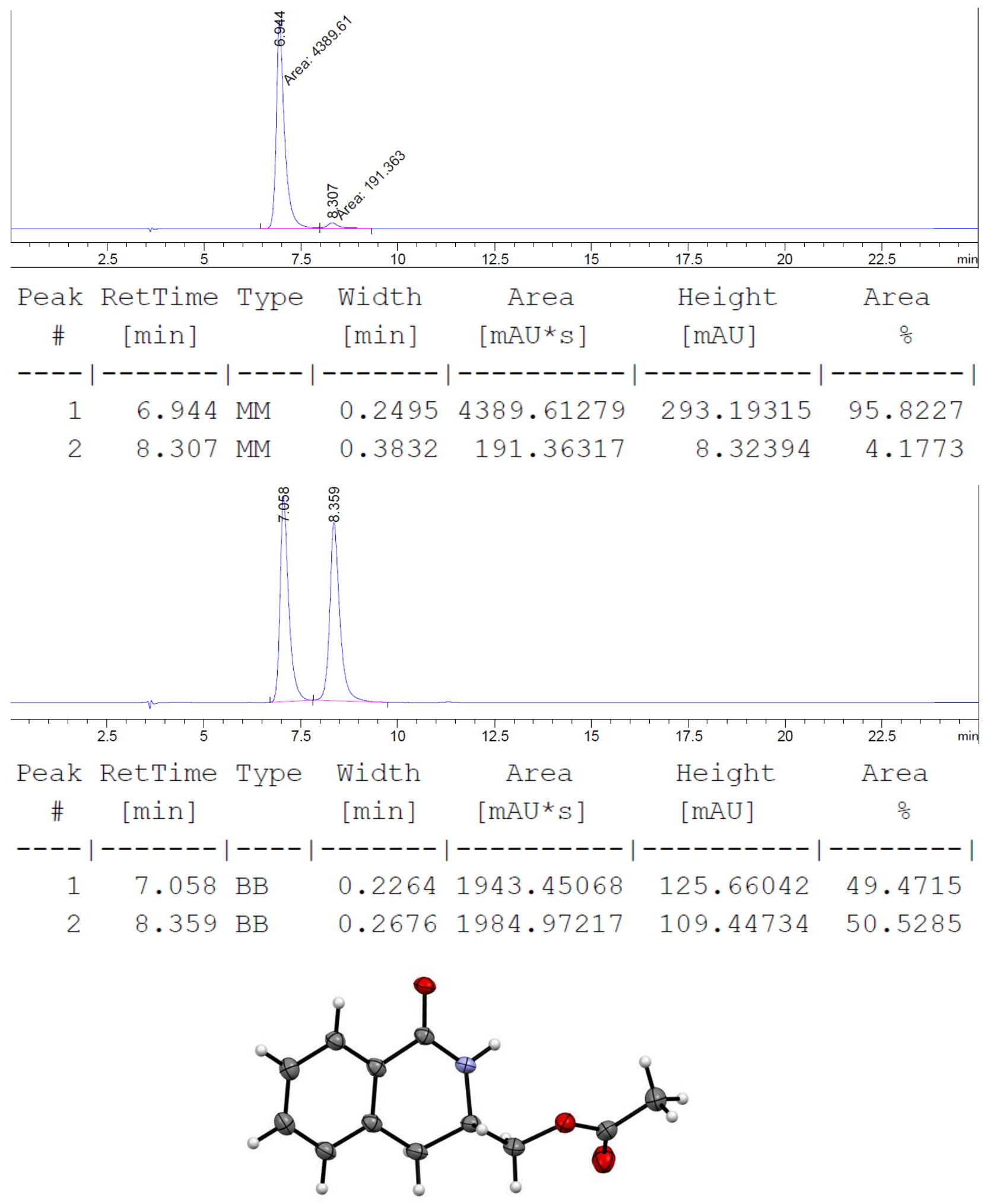

X-Ray structure of 3ao (CCDC No: 1898856). 
$(-)-(S, E)-3-(5-p h e n y \mid p e n t-1-e n-1-y l)-3,4-d i h y d r o i s o q u i n o l i n-1(2 H)$-one (3ap)

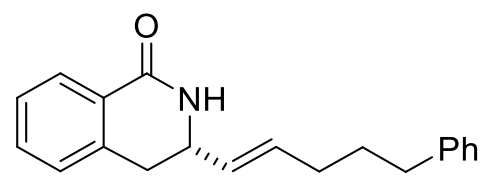

General procedure A. Yield: 76\% (44.0 mg, $152 \mu \mathrm{mol})$, white solid.

${ }^{1} \mathrm{H}-\mathrm{NMR}\left(400 \mathrm{MHz}, \mathrm{CDCl}_{3}\right) \quad \delta=8.07(\mathrm{dd}, J=7.7,0.8 \mathrm{~Hz}, 1 \mathrm{H}), 7.44$

(td, $J=7.5 \mathrm{~Hz}, 1.3 \mathrm{~Hz}, 1 \mathrm{H}), 7.34(\mathrm{t}, J=7.5 \mathrm{~Hz}, 1 \mathrm{H}), 7.31-7.26(\mathrm{~m}$, 2H), 7.22 - $7.12(\mathrm{~m}, 4 \mathrm{H}), 6.08(\mathrm{~s}, 1 \mathrm{H}), 5.74$ (dt, $J=15.2,6.7 \mathrm{~Hz}, 1 \mathrm{H}), 5.51$ (ddt, $J=15.2,7.3$, $1.1 \mathrm{~Hz}, 1 \mathrm{H}), 4.25-4.17(\mathrm{~m}, 1 \mathrm{H}), 3.00$ (dd, $J=15.6,4.8 \mathrm{~Hz}, 1 \mathrm{H}), 2.91$ (dd, $J=15.6,9.8 \mathrm{~Hz}, 1 \mathrm{H}$ ), $2.58\left(\mathrm{t}, J=7.6 \mathrm{~Hz}, 2 \mathrm{H}\right.$ ), $2.08\left(\mathrm{q}, J=7.1 \mathrm{~Hz}, 2 \mathrm{H}\right.$ ), 1.70 (quintet, $J=7.6 \mathrm{~Hz}, 2 \mathrm{H}$ ) ppm; ${ }^{13} \mathrm{C}\left\{{ }^{1} \mathrm{H}\right\} \mathbf{N M R}$ $\left(101 \mathrm{MHz}, \mathrm{CDCl}_{3}\right) \delta=166.2,142.2,137.8,133.9,132.4,129.7,128.6,128.5,128.4,128.0,127.5$, 127.2, 125.9, 53.8, 35.3, 35.2, 31.7, 30.7 ppm; IR (ATR): 3199, 3025, 2928, 1661, 1604, 1463, 1391, 1316, 1155, 967, 745, $699 \mathrm{~cm}^{-1} ; \boldsymbol{R}_{\mathrm{f}}=0.60$ (hexane/EtOAc = 1:2); $[\boldsymbol{\alpha}]_{\mathrm{D}}^{20}:-34.9(c=1.00$, $\mathrm{CHCl}_{3}$ ); HRMS (ESI/QTOF): calcd. for $\left[\mathrm{C}_{20} \mathrm{H}_{22} \mathrm{NO}^{+},[\mathrm{M}+\mathrm{H}]^{+}:\right.$292.1696; found 292.1701; Chiral HPLC: Chiralpak IC, 4.6 x $250 \mathrm{~mm} ; 30 \%$ i-PrOH/hexane, $1.0 \mathrm{~mL} / \mathrm{min}, 254 \mathrm{~nm}$; tr (major) = 18.7 $\min , \operatorname{tr}($ minor $)=26.7 \mathrm{~min}, 98.6 / 1.4$ er.

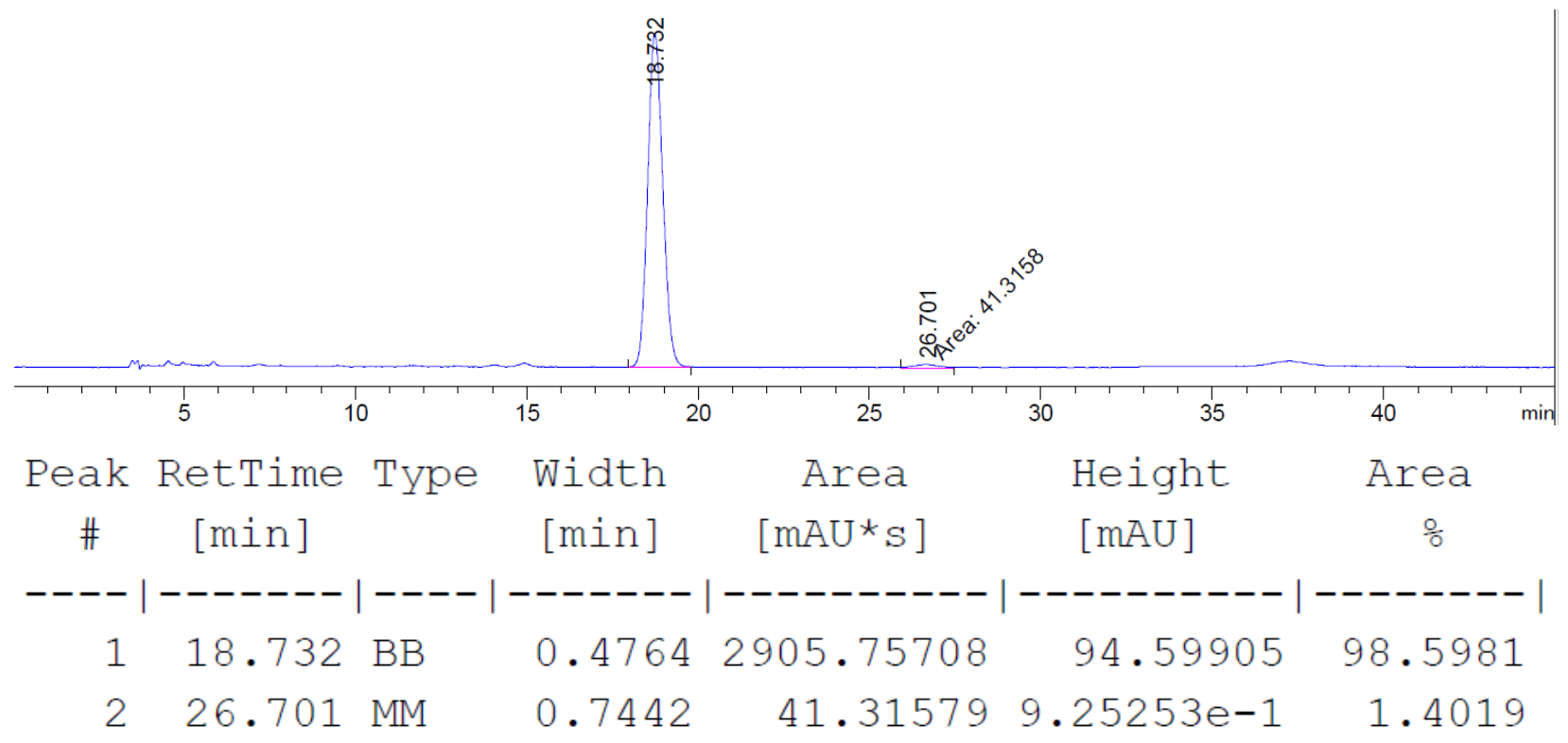




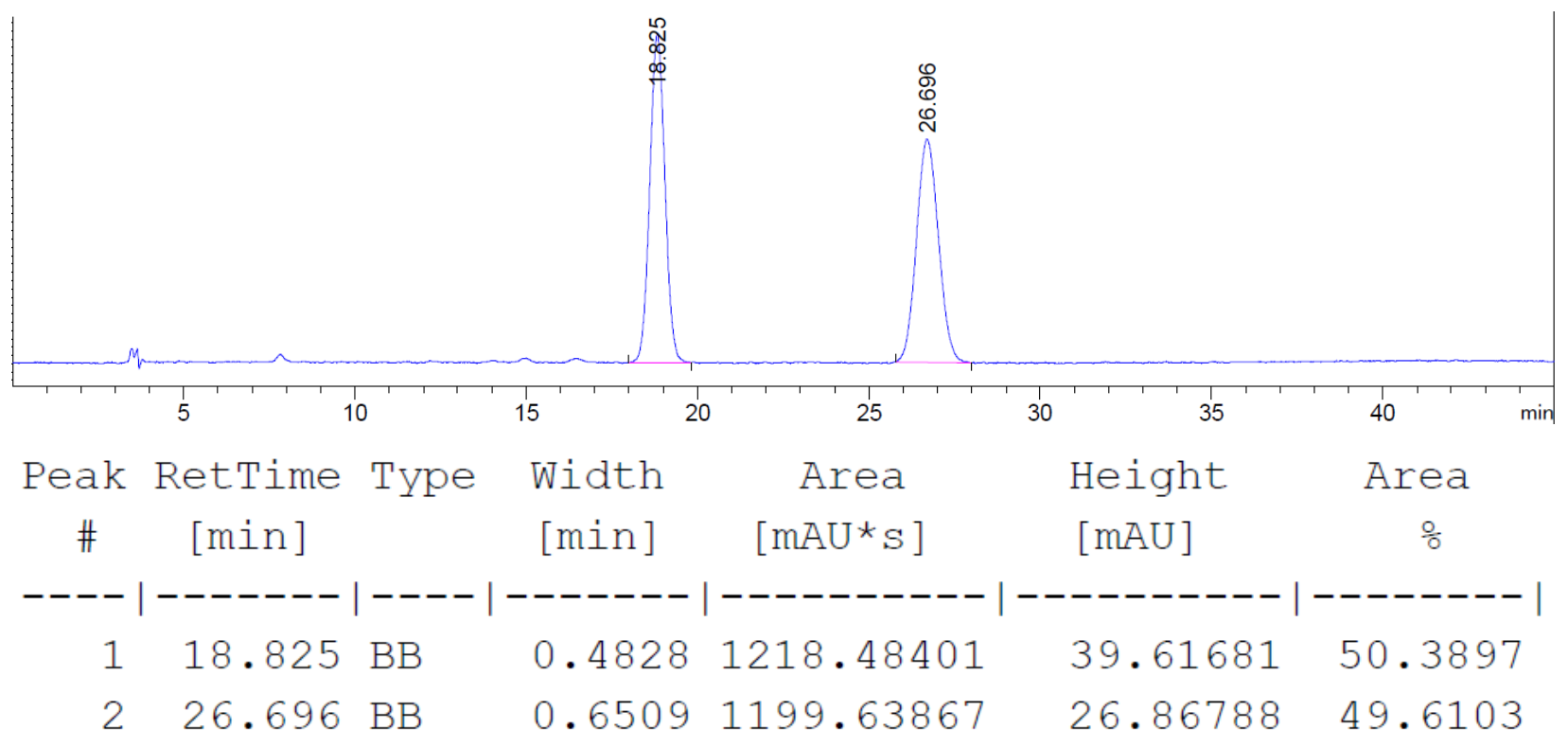

\section{(-)-(S)-3-(6-methylhepta-1,5-dien-2-yl)-3,4-dihydroisoquinolin-1(2H)-one (3aq)}

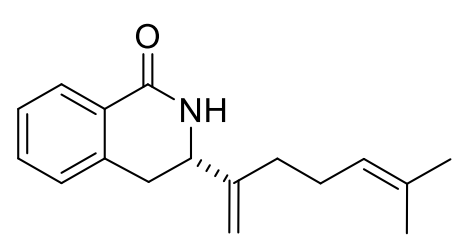

Cobalt catalyst Co7 (16.0 mg, $20.0 \mu \mathrm{mol}, 10.0 \mathrm{~mol} \%)$ was loaded into a flame-dried reaction vessel. In $\mathrm{N}_{2}$-filled glovebox, caesium pivalate (56.2 mg, $240 \mu \mathrm{mol}, 1.20$ equiv.), caesium carbonate (32.6 mg, 0.10 mmol, 0.50 equiv.) and silver triflate (10.3 mg, $40.0 \mu \mathrm{mol}, 20.0 \mathrm{~mol} \%)$

were added. The reaction vessel was sealed with a septum and dry, degassed HFIP (1.33 mL) was added. The resulting mixture was stirred at $20^{\circ} \mathrm{C}$ for $5 \mathrm{~min}$. Then, dry and degassed 1,2dichloroethane $(660 \mu \mathrm{L})$, myrcene $(600 \mu \mathrm{mol}, 3.00$ equiv.) and solid $\mathrm{N}$-chloroamide (200 $\mu \mathrm{mol}$, 1.00 equiv.) were added. The reaction mixture was stirred at $40^{\circ} \mathrm{C}$ for $18 \mathrm{~h}$ in the absence of light. The crude reaction mixture was filtered through a pad of silica gel, all volatiles were evaporated and the residue was purified by column chromatography on silica gel (gradient: EtOAc/pentane = $0 \% \rightarrow 50 \%$ ). Yield: $73 \%$ (37.3 mg, $146 \mu \mathrm{mol}$ ), yellow oil. ${ }^{1} \mathbf{H}$ NMR $\left(400 \mathrm{MHz}, \mathrm{CDCl}_{3}\right) \delta=8.07$ (dd, $J=7.7,1.0 \mathrm{~Hz}, 1 \mathrm{H}$ ), 7.45 (td, $J=7.5 \mathrm{~Hz}, 1.4 \mathrm{~Hz}, 1 \mathrm{H}), 7.35$ (t, $J=7.5 \mathrm{~Hz}, 1 \mathrm{H}$ ), 7.20 (d, $J=7.5 \mathrm{~Hz}$, $1 \mathrm{H}), 5.81(\mathrm{~s}, 1 \mathrm{H}), 5.15(\mathrm{~s}, 1 \mathrm{H}), 5.13-5.07(\mathrm{~m}, 1 \mathrm{H}), 5.01(\mathrm{~s}, 1 \mathrm{H}), 4.23(\mathrm{dd}, J=9.7,5.4 \mathrm{~Hz}, 1 \mathrm{H}), 3.05$ (dd, $J=15.7,5.4 \mathrm{~Hz}, 1 \mathrm{H}$ ), 2.99 (dd, $J=15.7,9.7 \mathrm{~Hz}, 1 \mathrm{H}$ ), 2.22-2.06 (m, 4H), 1.70 (s, 3H), 1.61 (s, 3H) ppm; ${ }^{13} \mathrm{C}\left\{{ }^{1} \mathrm{H}\right\}$ NMR $\left(101 \mathrm{MHz}, \mathrm{CDCl}_{3}\right) \delta=166.6,148.4,137.8,132.7,132.5,128.6,128.1$, 127.5, 127.3, 123.4, 112.0, 56.3, 34.3, 32.9, 26.7, 25.8, 17.9 ppm; IR (ATR): 3201, 3080, 2920, 1664, 1604, 1578, 1462, 1389, 1289, 1155, 901, 742, $529 \mathrm{~cm}^{-1} ; \boldsymbol{R}_{\mathrm{f}}: 0.5$ (hexane/EtOAc = 1/2); $[\alpha]_{D}^{20}:-108.2\left(c=1.00, \mathrm{CHCl}_{3}\right)$; HRMS (ESI/QTOF): calcd. for $\left[\mathrm{C}_{17} \mathrm{H}_{22} \mathrm{NO}^{+},[\mathrm{M}+\mathrm{H}]^{+}: 256.1696\right.$; found: 256.1705; Chiral HPLC: Chiralpak IC, $4.6 \times 250 \mathrm{~mm}$; 30\% i-PrOH/hexane, $1.0 \mathrm{~mL} / \mathrm{min}$, $254 \mathrm{~nm}$; tr (major) = $9.3 \mathrm{~min}, \operatorname{tr}($ minor $)=10.3 \mathrm{~min}, 99.5 / 0.5 \mathrm{er}$. 


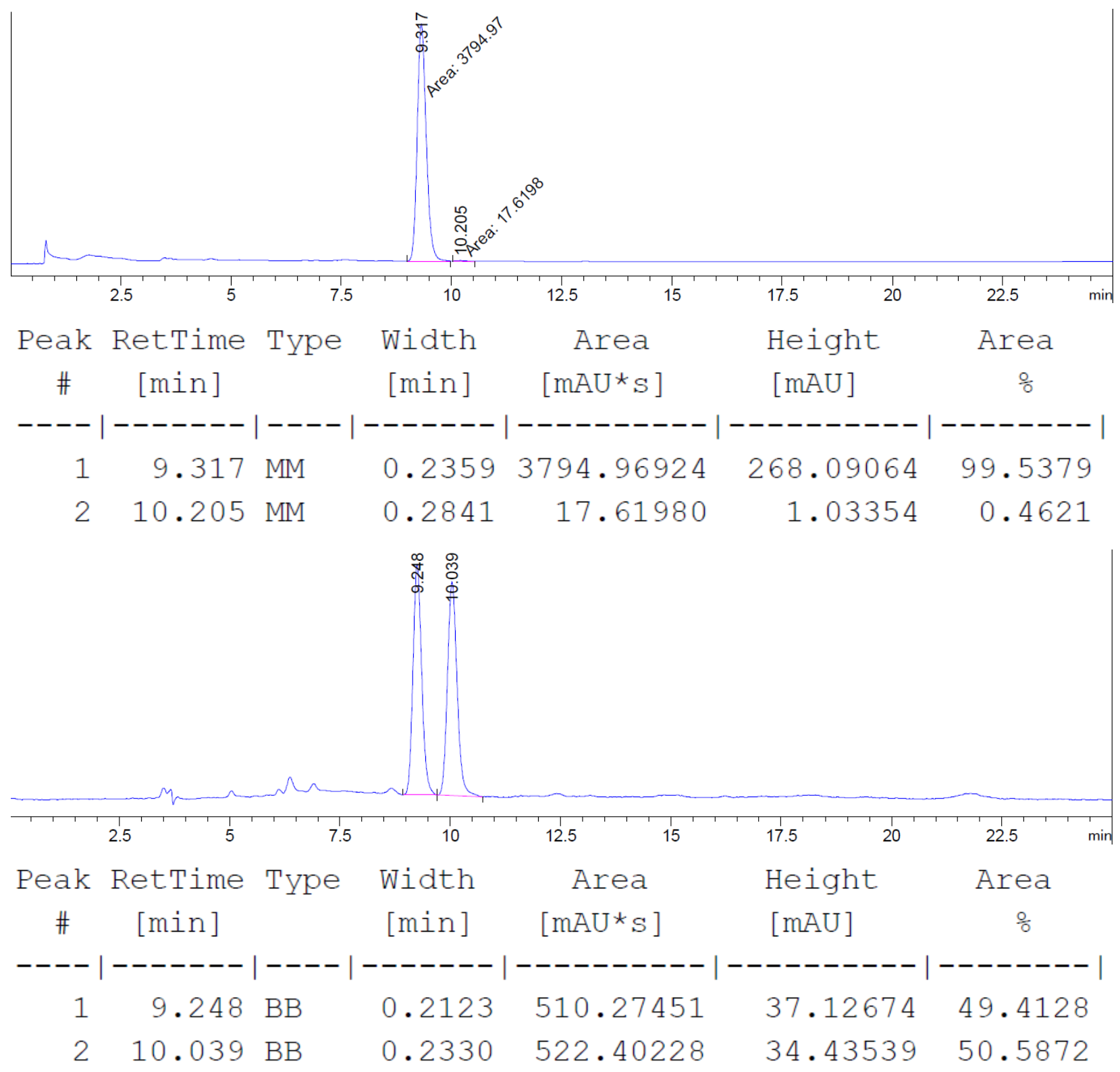

(-)-(4aR,10bR)-1,4a, 5,10b-tetrahydrophenanthridin-6(2H)-one (3ar)

General Procedure A. Yield: $74 \%(29.0 \mathrm{mg}, 147 \mu \mathrm{mol})$, white solid. The spectra
match with the literature values ${ }^{9}{ }^{1} \mathrm{H} \mathrm{NMR}\left(400 \mathrm{MHz}, \mathrm{CDCl}_{3}\right) \delta=8.08(\mathrm{dd}, J=7.8$, $-2.87(\mathrm{~m}, 1 \mathrm{H}), 2.27-2.18(\mathrm{~m}, 2 \mathrm{H}), 2.07-1.90(\mathrm{~m}, 1 \mathrm{H}), 1.75-1.65(\mathrm{~m}, 1 \mathrm{H}) \mathrm{ppm} ; \boldsymbol{R}_{\mathrm{f}}: 0.30$ (hexane/EtOAc $=1 / 1) ;[\alpha]_{D}^{20}:-38.8\left(c=1.00, \mathrm{CHCl}_{3}\right)$; Chiral HPLC: Chiralpak IA, 4.6 × $250 \mathrm{~mm}$; $20 \% \mathrm{i}-\mathrm{PrOH} /$ hexane, $1.0 \mathrm{~mL} / \mathrm{min}, 254 \mathrm{~nm}$; tr (major) $=7.4 \mathrm{~min}$, $\operatorname{tr}$ (minor) $=9.7 \mathrm{~min}, 86.5 / 13.5 \mathrm{er}$. 


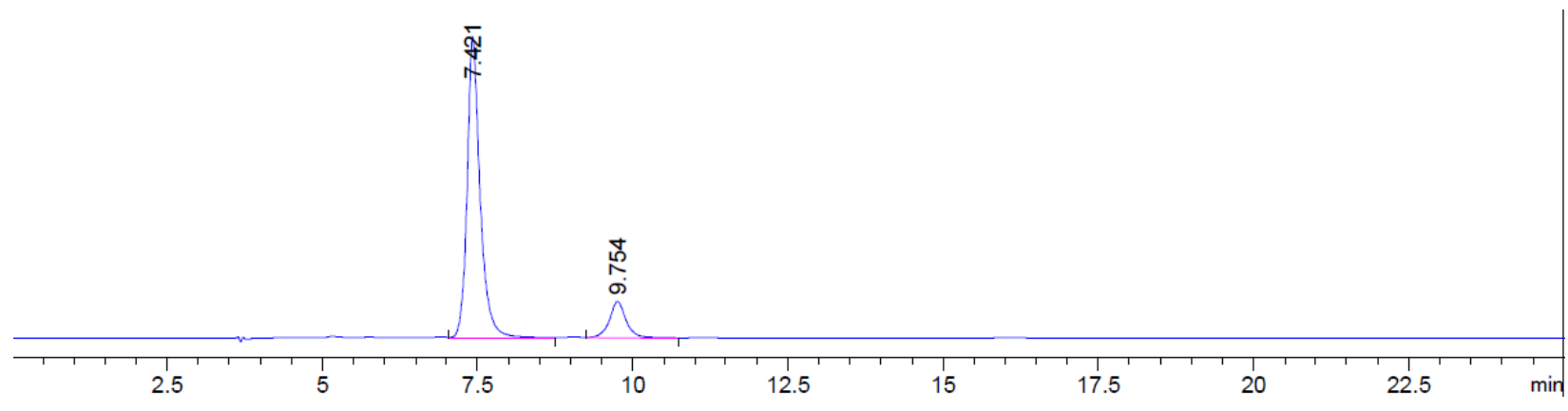

\begin{tabular}{|c|c|c|c|c|c|c|}
\hline $\begin{array}{c}\text { eak } \\
\#\end{array}$ & $\begin{array}{c}\text { RetTime } \\
\text { [min] }\end{array}$ & Type & $\begin{array}{l}\text { Width } \\
\text { [min] }\end{array}$ & $\begin{array}{c}\text { Area } \\
{\left[\mathrm{mAU}^{*} \mathrm{~s}\right]}\end{array}$ & $\begin{array}{l}\text { Height } \\
{[\mathrm{mAU}]}\end{array}$ & $\begin{array}{c}\text { Area } \\
\frac{8}{8}\end{array}$ \\
\hline & & & & & & \\
\hline 1 & & 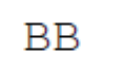 & & 4170 . & 327 & 64 \\
\hline 2 & 9.754 & $\mathrm{BB}$ & & 648.36328 & 34.88499 & 4536 \\
\hline
\end{tabular}

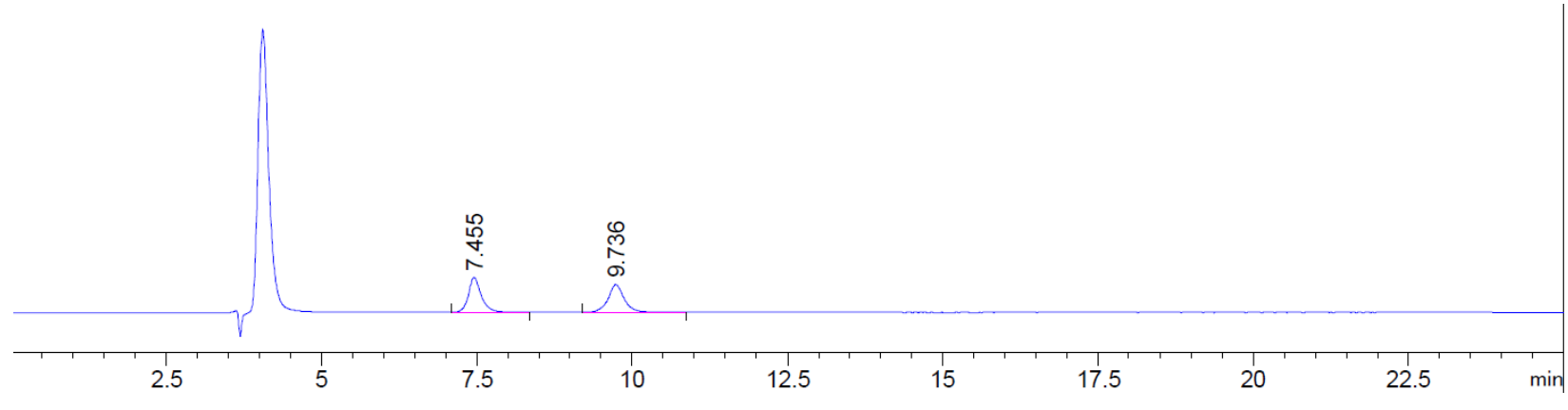

\begin{tabular}{|c|c|c|c|c|c|c|}
\hline $\begin{array}{c}\text { eak } \\
\text { \# }\end{array}$ & $\begin{array}{c}\text { RetTime } \\
\text { [min] }\end{array}$ & Type & $\begin{array}{l}\text { Width } \\
\text { [min] }\end{array}$ & $\begin{array}{c}\text { Area } \\
{\left[\mathrm{mAU}^{*} \mathrm{~s}\right]}\end{array}$ & $\begin{array}{l}\text { Height } \\
{[\mathrm{mAU}]}\end{array}$ & $\begin{array}{c}\text { Area } \\
\frac{\circ}{0}\end{array}$ \\
\hline & & & & 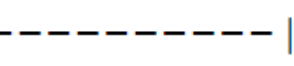 & --- & \\
\hline 1 & & & & & 10 & 56 \\
\hline 2 & 9.736 & & & 599.15784 & 32.45769 & 3644 \\
\hline
\end{tabular}

\section{$\underline{(+)-(6 \mathrm{a} R, 12 \mathrm{a} R, \mathrm{Z})-6 \mathrm{a}, 7,8,11,12,12 \mathrm{a} \text {-hexahydrocycloocta[c]isoquinolin-5(6H)-one (3as) }}$}

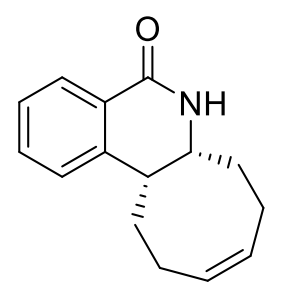

General Procedure A. Yield: 53\% (24.0 mg, $106 \mu \mathrm{mol})$, white solid. The spectra match with the literature values. ${ }^{8} \mathrm{H} \mathrm{HMR}\left(400 \mathrm{MHz}, \mathrm{CDCl}_{3}\right) \delta=8.05$ (d, $J=7.7$ $\mathrm{Hz}, 1 \mathrm{H}), 7.45(\mathrm{td}, J=7.5,1.6 \mathrm{~Hz}, 1 \mathrm{H}), 7.34(\mathrm{td}, J=7.5,1.3 \mathrm{~Hz}, 1 \mathrm{H}), 7.22-7.15$ $(\mathrm{m}, 1 \mathrm{H}), 5.88(\mathrm{~s}, 1 \mathrm{H}), 5.74-5.56(\mathrm{~m}, 2 \mathrm{H}), 4.00-3.91(\mathrm{~m}, 1 \mathrm{H}), 3.25(\mathrm{dd}, J=$ $12.2,4.0 \mathrm{~Hz}, 1 \mathrm{H}), 2.90-2.73(\mathrm{~m}, 1 \mathrm{H}), 2.68-2.55(\mathrm{~m}, 1 \mathrm{H}), 2.53-2.40(\mathrm{~m}, 1 \mathrm{H}), 2.26-2.03(\mathrm{~m}$, $3 \mathrm{H}$ ), 1.86 (ddd, $J=14.0,6.7,3.2 \mathrm{~Hz}, 1 \mathrm{H}$ ), 1.58 (ddt, $J=9.4,7.2,4.7 \mathrm{~Hz}, 1 \mathrm{H}$ ) ppm; $\boldsymbol{R}_{\mathrm{f}}: 0.26$ (hexane/EtOAc $=1 / 1) ;[\alpha]_{D}^{20}:+46.9\left(c=1.00, \mathrm{CHCl}_{3}\right)$; Chiral HPLC: Chiralpak IC, 4.6 × $250 \mathrm{~mm}$; 
$30 \% \mathrm{i}-\mathrm{PrOH} /$ hexane, $1.0 \mathrm{~mL} / \mathrm{min}, 286 \mathrm{~nm}$; $\operatorname{tr}$ (minor) = $13.4 \mathrm{~min}$, $\operatorname{tr}$ (major) = $20.3 \mathrm{~min}, 87.5 / 12.5$ er.

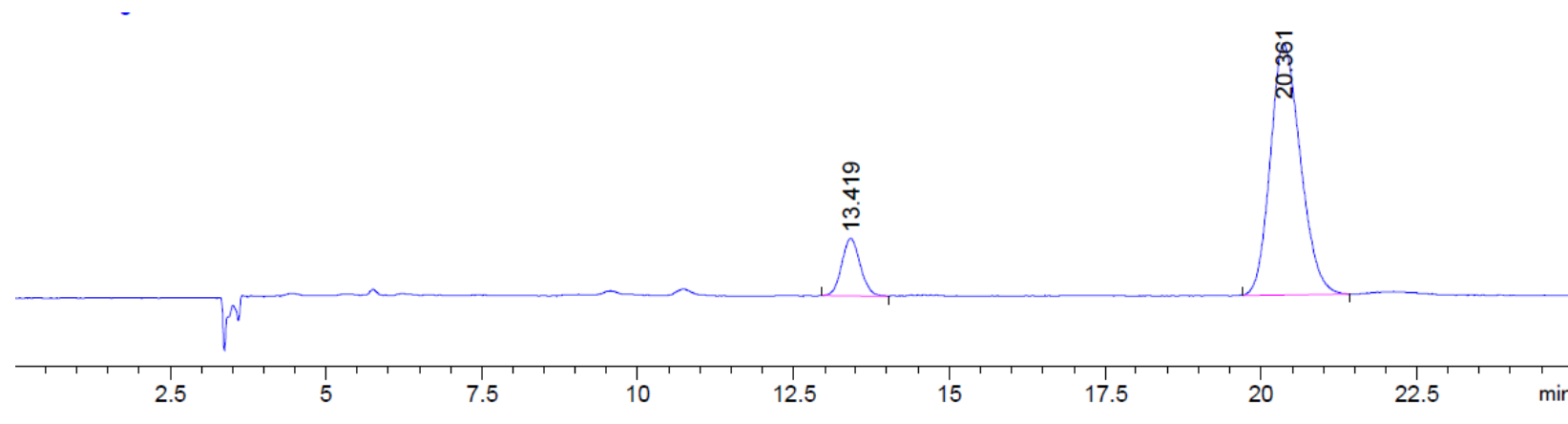

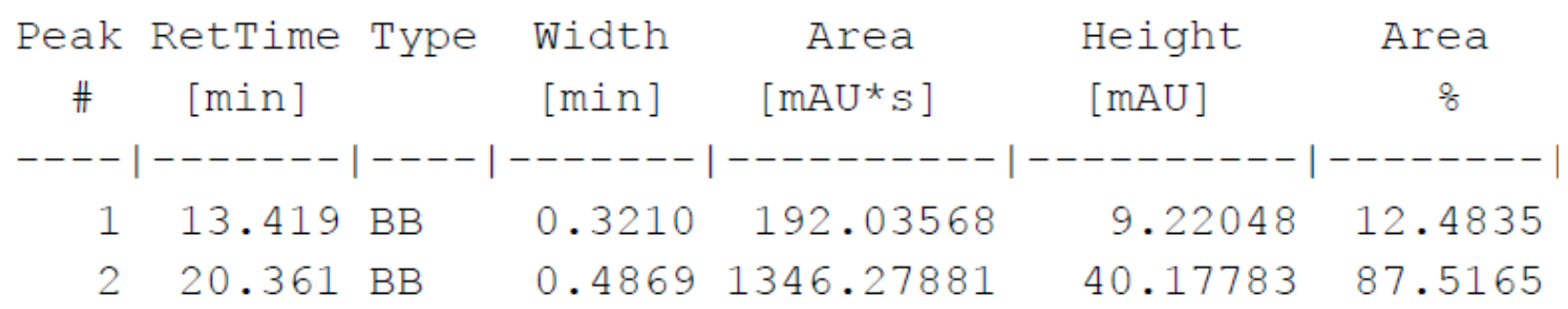

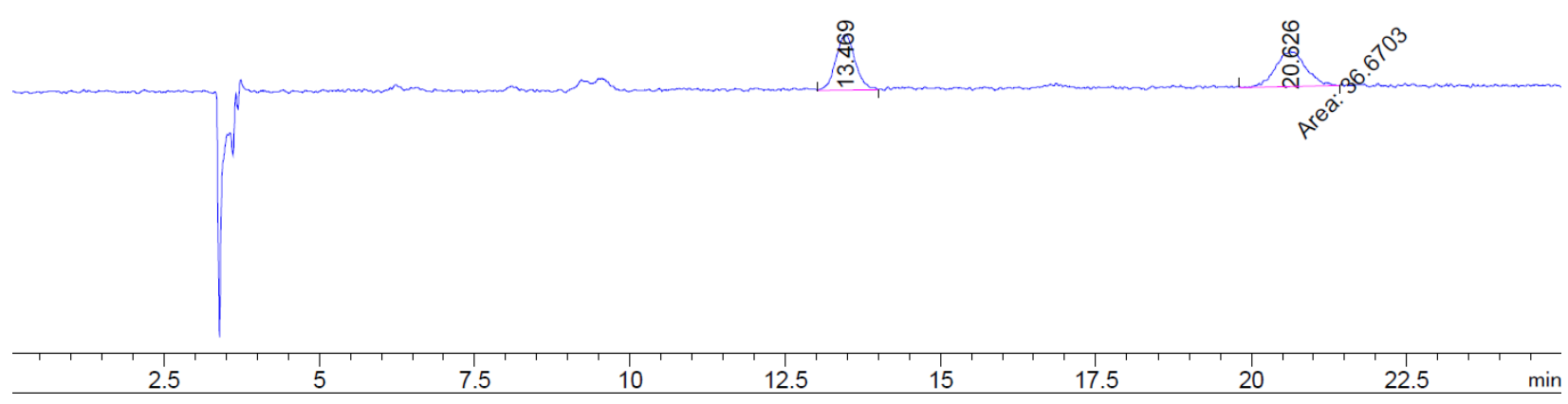

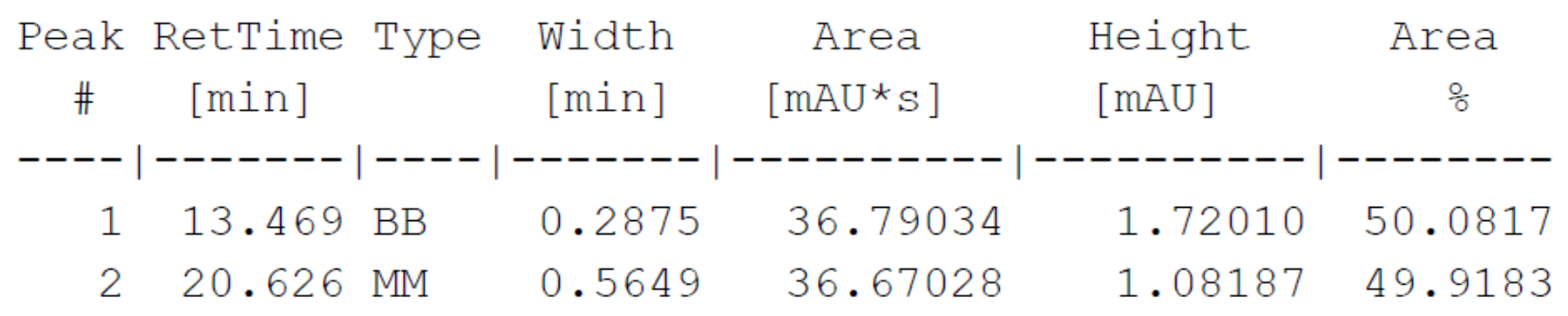


(-)-(6aS,7R,12R,12a R)-6a,7,12,12a-tetrahydro-7,12-epoxybenzo[b]phenanthridin-5(6H)one (3at)<smiles>O=C1N[C@@]2(Cc3ccccc3[C@@H]2O)c2ccccc21</smiles>

General Procedure A. Yield: $91 \%$ (48.0 mg, $182 \mu \mathrm{mol})$, white solid. The spectra match with the literature values. ${ }^{14} 1 \mathrm{H}$ NMR $\left(400 \mathrm{MHz}, \mathrm{CDCl}_{3}\right) \delta=8.20$ (dd, $J=$ 7.8, $1.4 \mathrm{~Hz}, 1 \mathrm{H}$ ), 7.58 (td, $J=7.6,7.5,1.4 \mathrm{~Hz}, 1 \mathrm{H}), 7.45-7.37$ (m, 4H), $7.34-$ $7.20(\mathrm{~m}, 2 \mathrm{H}), 7.08(\mathrm{~s}, 1 \mathrm{H}), 5.34(\mathrm{~s}, 1 \mathrm{H}), 5.27(\mathrm{~s}, 1 \mathrm{H}), 4.02(\mathrm{~d}, J=8.5 \mathrm{~Hz}, 1 \mathrm{H})$, 3.40 (d, $J=8.4 \mathrm{~Hz}, 1 \mathrm{H}$ ) ppm; IR (ATR): 3674, 3662, 2987, 2971, 2901, 1669, 1473, 1406, 1382, 1257, 1066, 1056, 857, 755, $656 \mathrm{~cm}^{-1}$; ; HRMS (ESI): calcd. for $\left[\mathrm{C}_{17} \mathrm{H}_{14} \mathrm{NO}_{2}\right]^{+},\left[\mathrm{M}+\mathrm{H}^{+}\right]^{+}: 264.1019$; found: 264.1019; $\boldsymbol{R}: 0.28$ (hexane/EtOAc = 1/1); [a] $]_{D}^{20}:-33.5$ $\left(c=1.00, \mathrm{CHCl}_{3}\right)$; Chiral HPLC: Chiralpak IB, $4.6 \times 250 \mathrm{~mm} ; 20 \% i-\mathrm{PrOH} / \mathrm{hexane}, 1.0 \mathrm{~mL} / \mathrm{min}$, $210 \mathrm{~nm}$; tr (minor) $=20.1 \mathrm{~min}$, tr $($ major $)=35.0 \mathrm{~min}, 97.8 / 2.2 \mathrm{er}$.

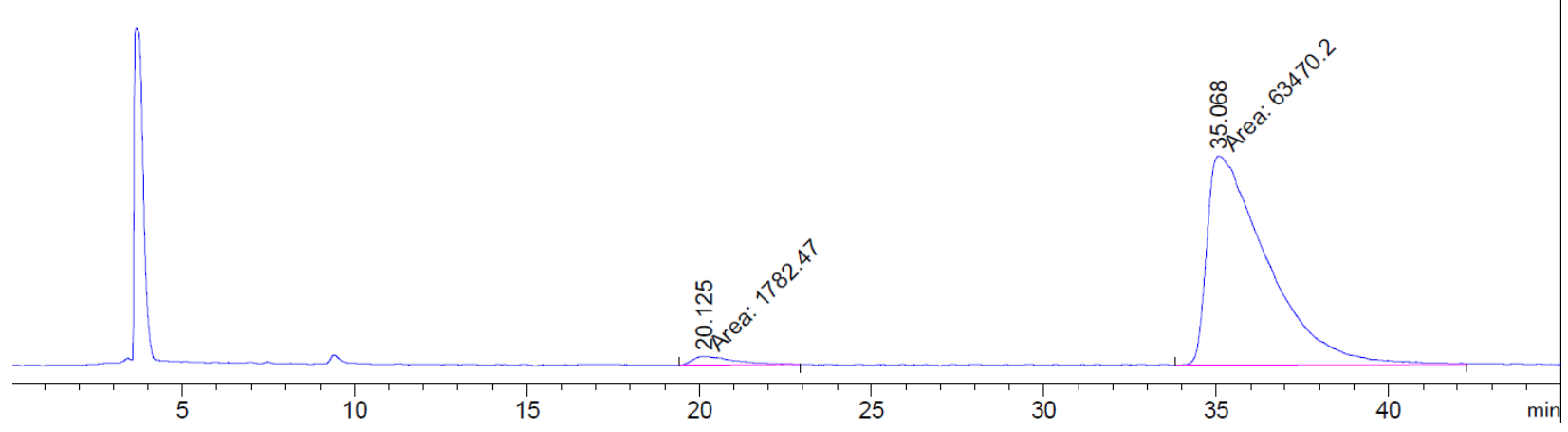

\begin{tabular}{|c|c|c|c|c|c|c|}
\hline $\begin{array}{c}\text { eak } \\
\#\end{array}$ & $\begin{array}{c}\text { RetTime } \\
\text { [min] }\end{array}$ & Type & $\begin{array}{l}\text { Width } \\
\text { [min] }\end{array}$ & $\begin{array}{c}\text { Area } \\
{\left[\mathrm{mAU}^{*} \mathrm{~s}\right]}\end{array}$ & $\begin{array}{l}\text { Height } \\
\text { [mAU] }\end{array}$ & $\begin{array}{c}\text { Area } \\
\frac{\circ}{0}\end{array}$ \\
\hline & & & & -------- & & \\
\hline 1 & & & & 199. & 583 & 767 \\
\hline 2 & & & 61 & 8944.35742 & 85.10497 & 8233 \\
\hline
\end{tabular}



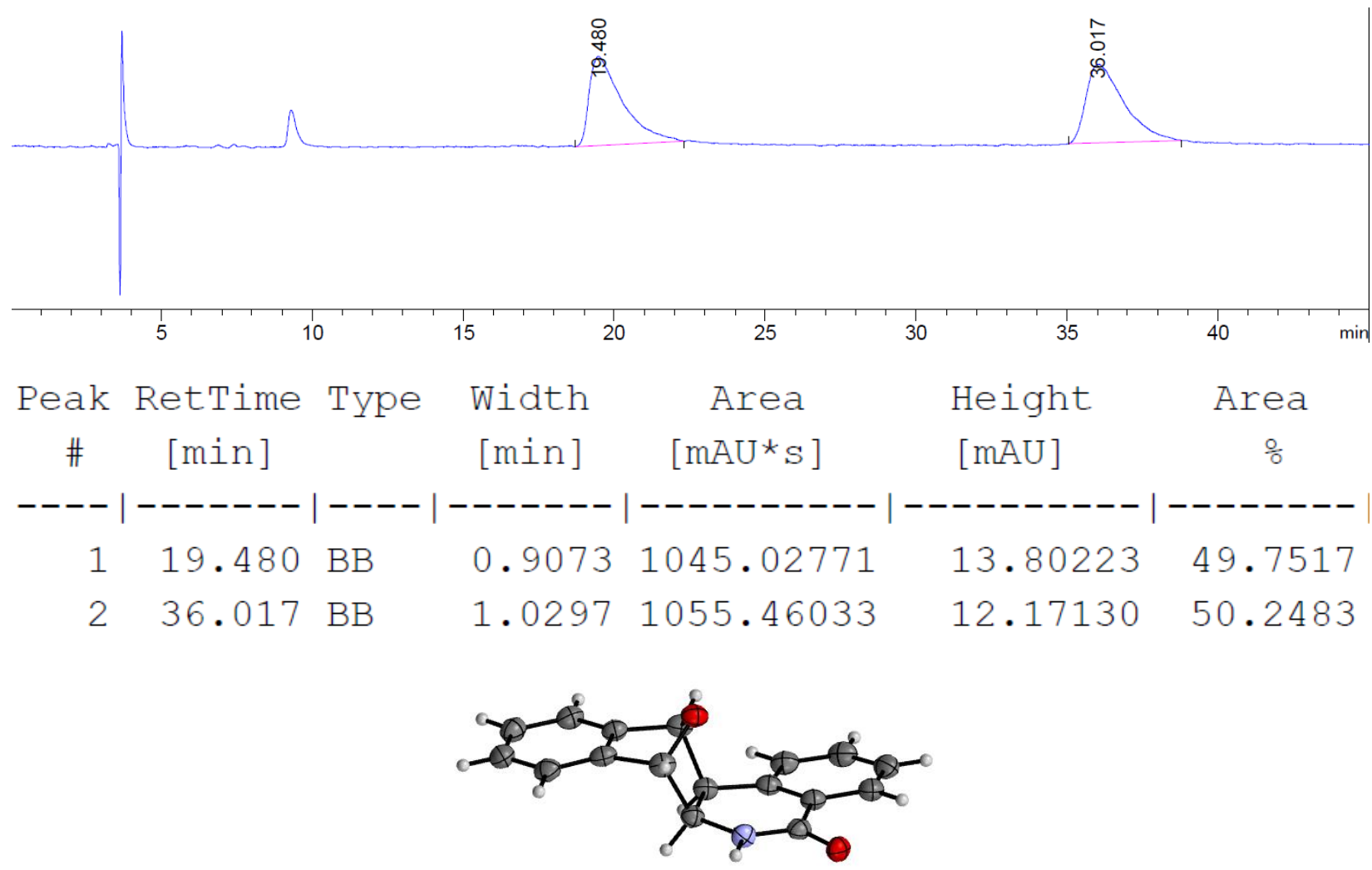

X-Ray structure of 3at (CCDC No: 1898857).

(+)-phenyl $\quad(6 \mathrm{a} S, 12 \mathrm{a} R)-5$-oxo-5,6,6a,7,12,12a-hexahydro-7,12-epiminobenzo[b]phenanthridine-13-carboxylate (3au)

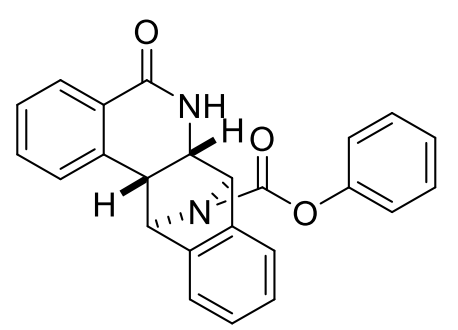

General procedure A. The alkene was added as a solid. Yield: $85 \%$ (65.0 mg, $170 \mu \mathrm{mol})$, white foam. ${ }^{1} \mathrm{H}$ NMR $\left(600 \mathrm{MHz}, \mathrm{CD}_{3} \mathrm{CN}, 70^{\circ} \mathrm{C}\right)$ $\delta=8.08(\mathrm{~d}, J=7.7 \mathrm{~Hz}, 1 \mathrm{H}), 7.67(\mathrm{~d}, J=7.7 \mathrm{~Hz}, 1 \mathrm{H}), 7.62(\mathrm{td}, J=7.3$, $0.8 \mathrm{~Hz}, 1 \mathrm{H}), 7.58(\mathrm{~d}, J=6.9 \mathrm{~Hz}, 1 \mathrm{H}), 7.49(\mathrm{~d}, J=7.0 \mathrm{~Hz}, 1 \mathrm{H}), 7.41(\mathrm{t}$, $J=7.6 \mathrm{~Hz}, 1 \mathrm{H}), 7.36-7.30(\mathrm{~m}, 2 \mathrm{H}), 7.27(\mathrm{t}, J=7.7 \mathrm{~Hz}, 2 \mathrm{H}), 7.15(\mathrm{t}, J$ $=7.4 \mathrm{~Hz}, 1 \mathrm{H}), 6.77(\mathrm{~s}, 2 \mathrm{H}), 6.60(\mathrm{~s}, 1 \mathrm{H}), 5.36(\mathrm{~s}, 2 \mathrm{H}), 4.00(\mathrm{dd}, J=8.5,1.8 \mathrm{~Hz}, 1 \mathrm{H}), 3.48(\mathrm{~d}, J=$ $8.5 \mathrm{~Hz}, 1 \mathrm{H}) \mathrm{ppm} ;{ }^{13} \mathrm{C}\left\{{ }^{1} \mathrm{H}\right\}$ NMR $\left(150 \mathrm{MHz}, \mathrm{CD}_{3} \mathrm{CN}, 70{ }^{\circ} \mathrm{C}\right) \delta=163.6,154.0,152.2,146.2,143.0$, 139.0, 133.8, 130.33, 130.31, 129.0, 128.7, 128.5, 128.4, 127.9, 126.6, 122.7, 122.4, 121.5, 71.7, 71.2, 57.7, 43.5 ppm; IR (ATR): 3272, 3063, 1721, 1670, 1602, 1474, 1400, 1337, 1201, 1164 , 1047, 751; $\boldsymbol{R}_{\mathfrak{f}}=0.6$ (toluene/ $\left.i-\mathrm{PrOH}=10: 1\right) ;[\alpha]_{D}^{20}:+7.33\left(c=0.50, \mathrm{CHCl}_{3}\right)$; HRMS (ESI/QTOF): calcd. for $\left[\mathrm{C}_{24} \mathrm{H}_{18} \mathrm{~N}_{2} \mathrm{NaO}_{3}\right]^{+}$, [M+Na] $]^{+}: 405.1210$; found 405.1212; Chiral HPLC: Chiralpak IA, 4.6 x $250 \mathrm{~mm} ; 40 \% \mathrm{i}-\mathrm{PrOH} /$ hexane, $1.0 \mathrm{~mL} / \mathrm{min}, 254 \mathrm{~nm}$; $\operatorname{tr}$ (minor) = $14.0 \mathrm{~min}, \operatorname{tr}$ (major) = $20.4 \mathrm{~min}$, $96.4 / 3.6 \mathrm{er}$. 

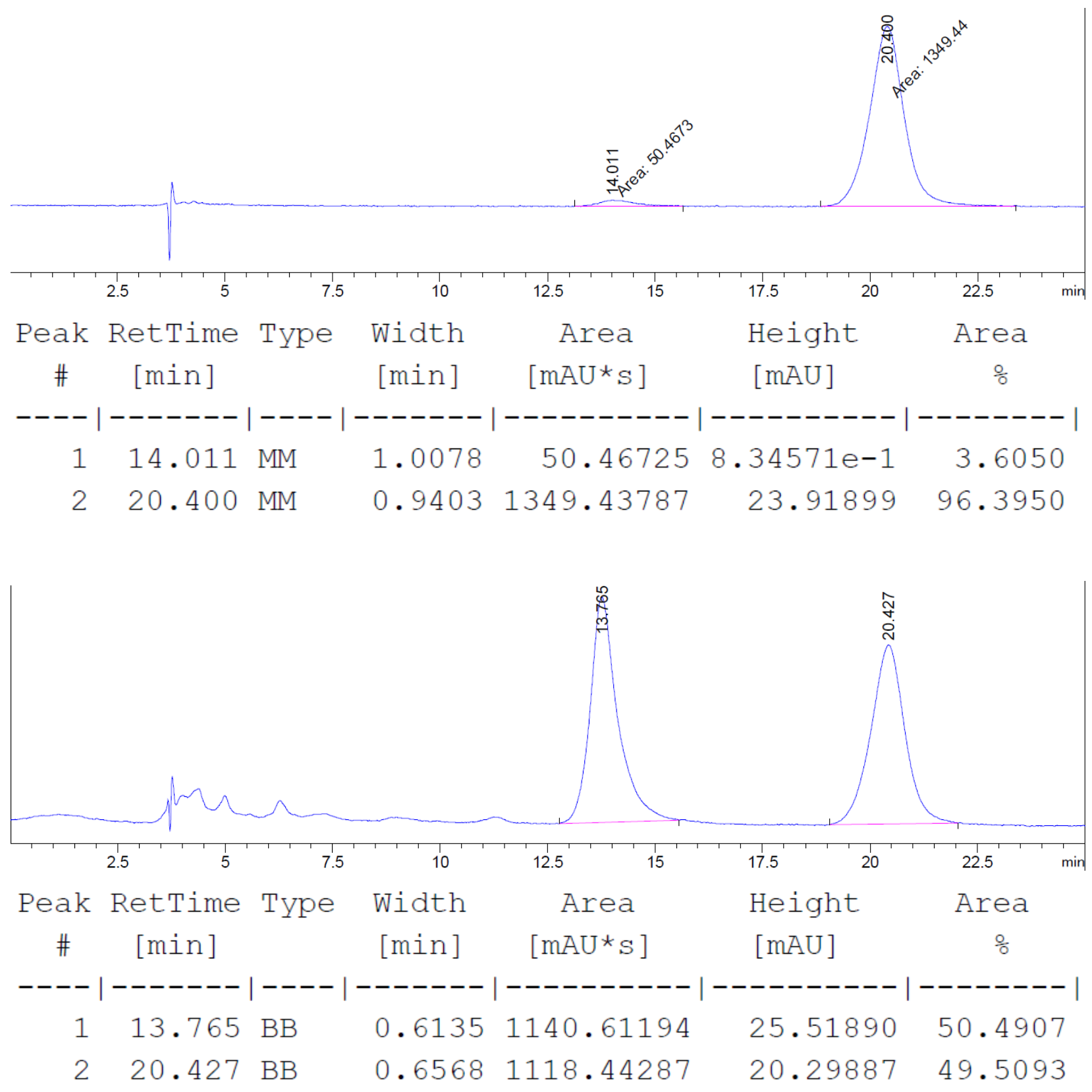


\section{Steric Maps}

Buried volumes and steric maps were generated with SambVca 2.0. ${ }^{15}$

Co2
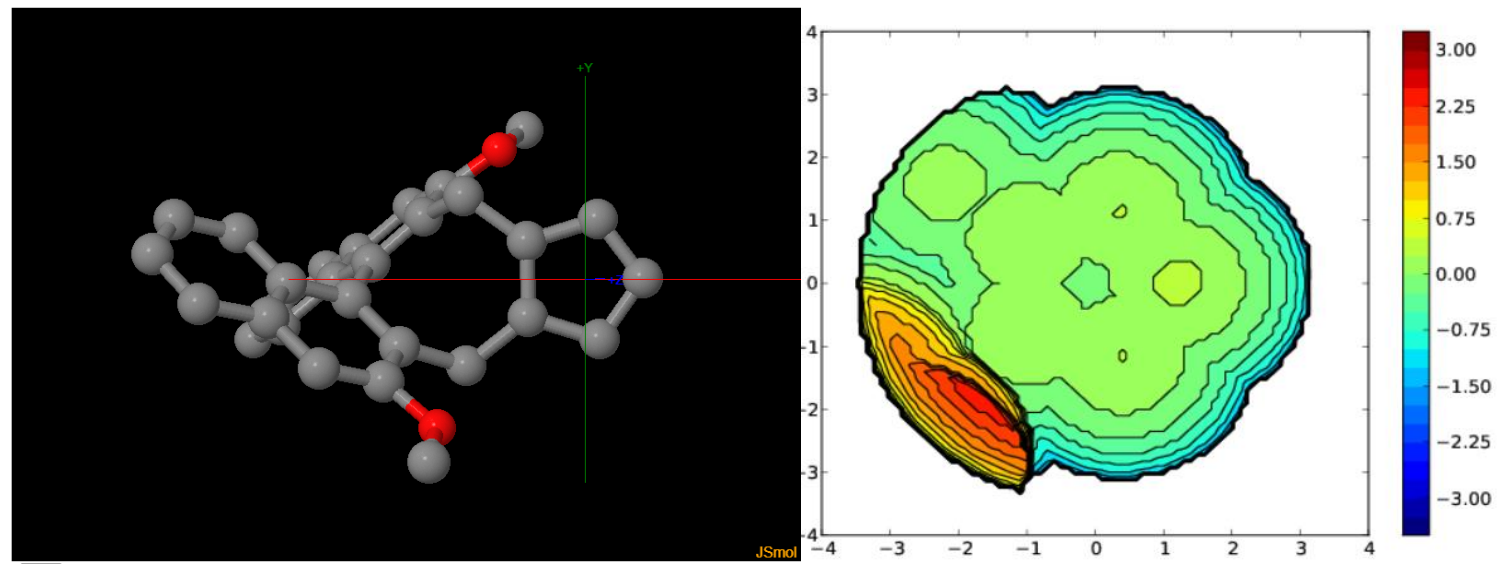

\begin{tabular}{|llcc}
\hline V Free & V Buried & V Total & V Exact \\
\hline 93.0 & 86.5 & 179.5 & 179.6 \\
\hline
\end{tabular}

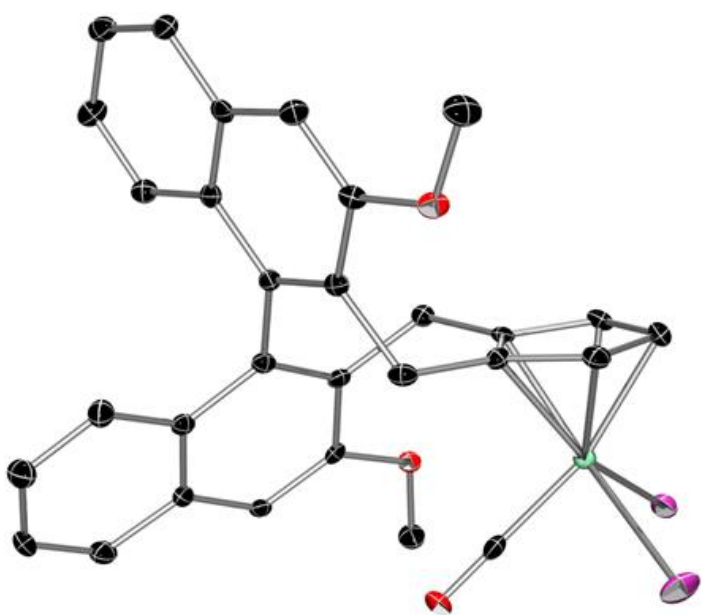

Co4
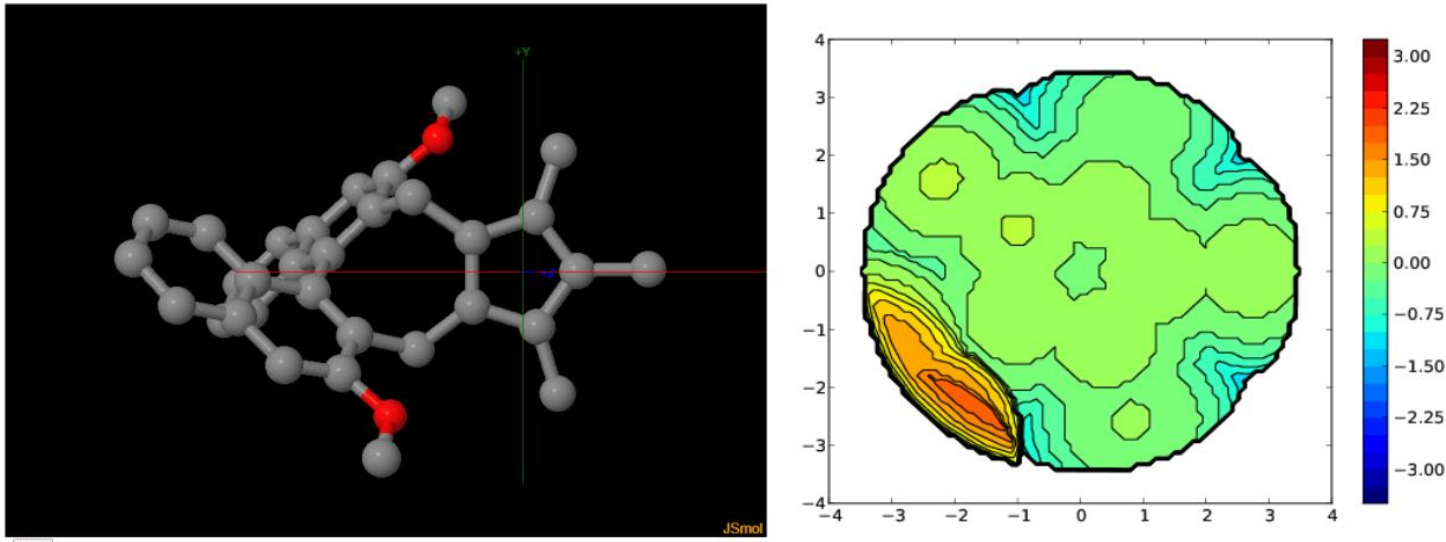


\begin{tabular}{|llcc|}
\hline V Free & V Buried & V Total & V Exact \\
\hline 86.1 & 93.4 & 179.5 & 179.6 \\
\hline
\end{tabular}

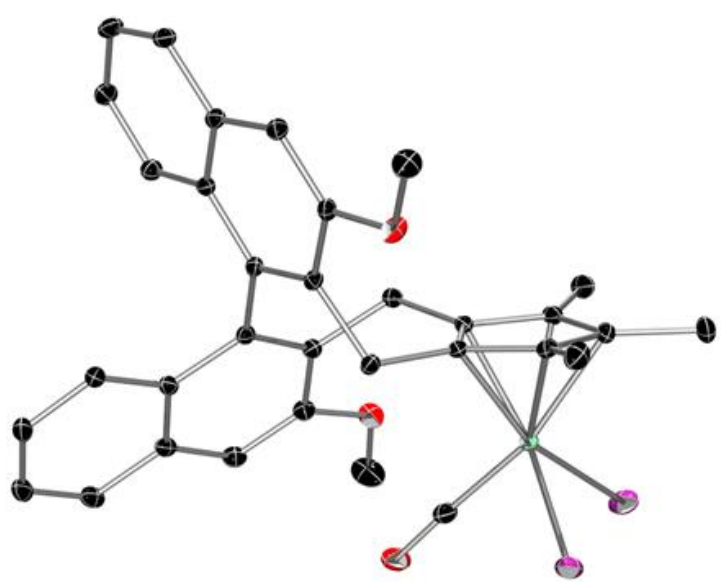

\section{Co6}
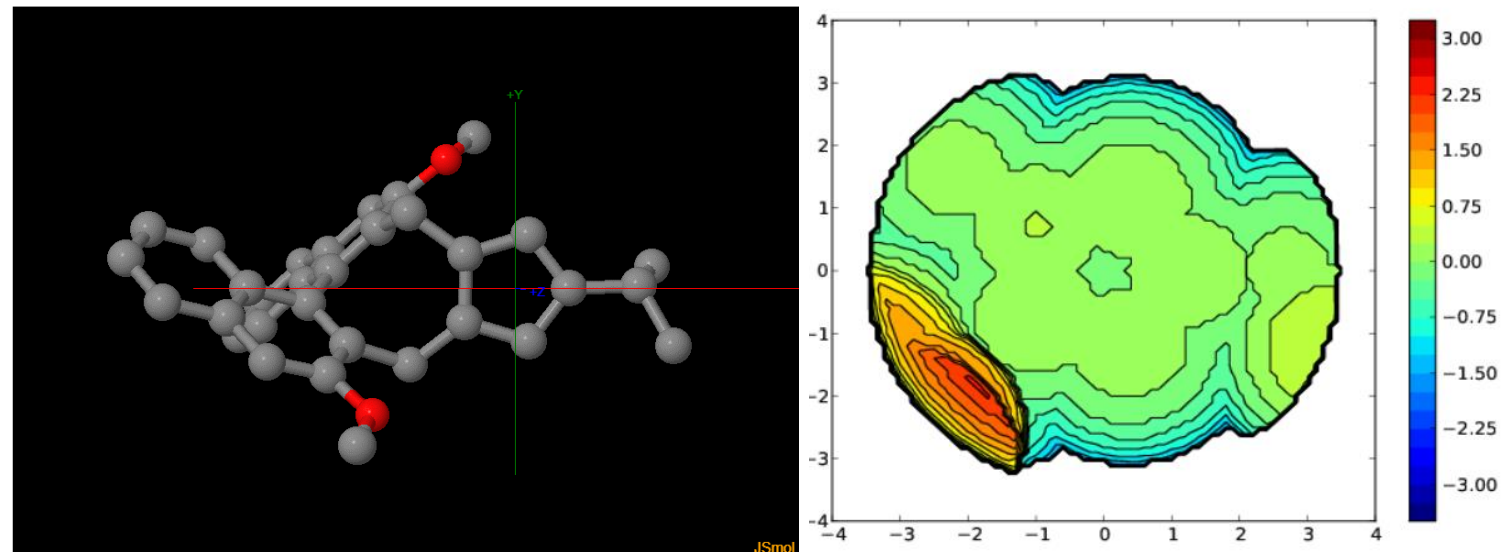

\begin{tabular}{|llcc|}
\hline V Free & V Buried & V Total & V Exact \\
\hline 89.3 & 90.3 & 179.5 & 179.6 \\
\hline
\end{tabular}

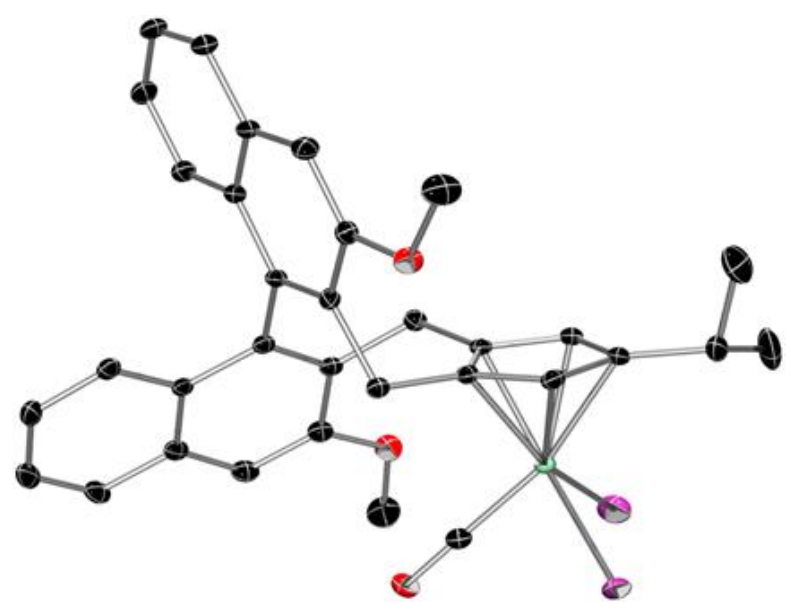




\section{Co7}
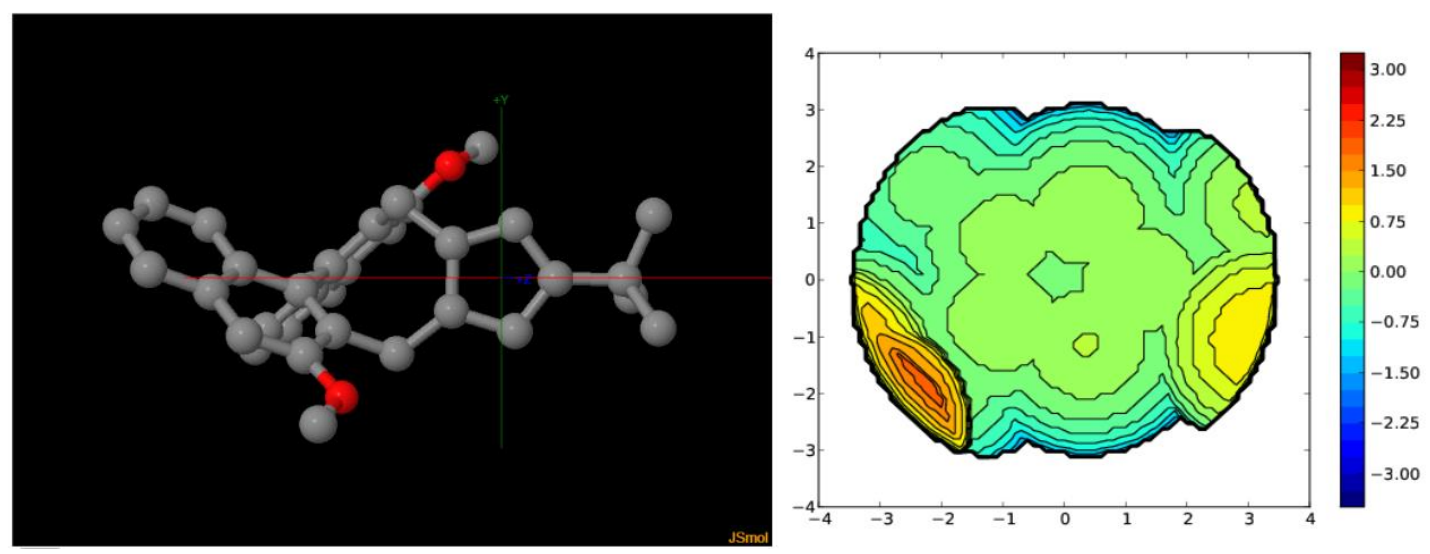

\begin{tabular}{|lccc|}
\hline V Free & V Buried & V Total & V Exact \\
\hline 89.4 & 90.1 & 179.5 & 179.6 \\
\hline
\end{tabular}

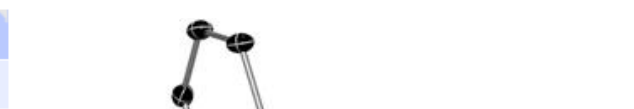

\begin{tabular}{|lll}
\hline \%V_Free & \%V_Bur & \% Tot/Ex \\
\hline 49.8 & 50.2 & 100.0 \\
\hline
\end{tabular}

\begin{tabular}{lccccc} 
Quadrant & V_f & V_b & V_t $_{-}$ & $\%_{\text {V_f }}$ & $\%$ V_b $_{-}$ \\
\hline SW & 20.1 & 24.8 & 44.9 & 44.8 & 55.2 \\
NW & 24.6 & 20.3 & 44.9 & 54.8 & 45.2 \\
NE & 23.2 & 21.7 & 44.8 & 51.7 & 48.3 \\
SE & 21.5 & 23.3 & 44.9 & 48.0 & 52.0 \\
\hline
\end{tabular}

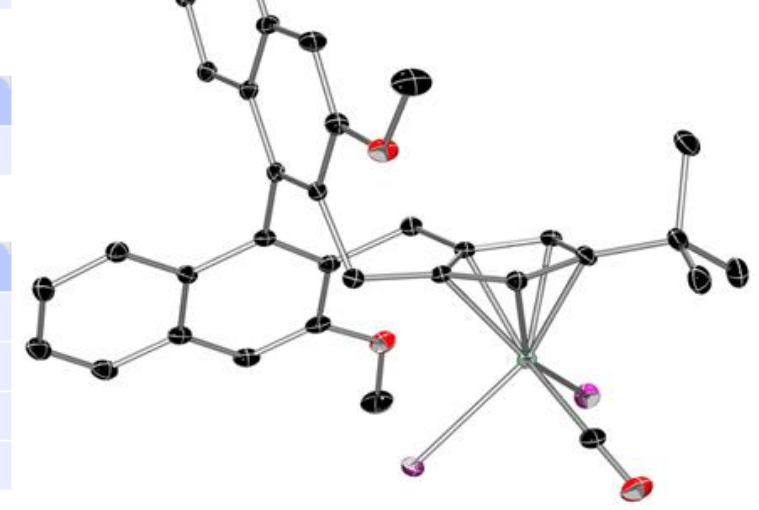




\section{References}

(1) Wang, S.-G.; Park, S. H.; Cramer, N. A Readily Accessible Class of Chiral Cp Ligands and their Application in Rull-Catalyzed Enantioselective Syntheses of Dihydrobenzoindoles Angew. Chem., Int. Ed. 2018, 57, 5459.

(2) Ye, B.; Cramer, N. A Tunable Class of Chiral Cp Ligands for Enantioselective Rhodium(III)-Catalyzed C-H Allylations of Benzamides J. Am. Chem. Soc. 2013, 135, 636.

(3) Smits, G.; Audic, B.; Wodrich, M. D.; Corminboeuf, C.; Cramer, N. A $\beta$-Carbon elimination strategy for convenient in situ access to cyclopentadienyl metal complexes Chem. Sci. 2017, 8, 7174.

(4) Audic, B.; Wodrich, M. D.; Cramer, N. Mild complexation protocol for chiral Cp×Rh and Ir complexes suitable for in situ catalysis Chem. Sci. 2019, 10, 781.

(5) Sun, Y.; Cramer, N. Tailored trisubstituted chiral $\mathrm{Cp}^{\times} \mathrm{Rh}^{111}$ catalysts for kinetic resolutions of phosphinic amides Chem. Sci. 2018, 9, 2981.

(6) Sun, B.; Yoshino, T.; Matsunaga, S.; Kanai, M. Air-Stable Carbonyl(pentamethylcyclopentadienyl)cobalt Diiodide Complex as a Precursor for Cationic (Pentamethylcyclopentadienyl)cobalt(III) Catalysis: Application for Directed C-2 Selective C-H Amidation of Indoles Adv. Synth. Catal. 2014, 356, 1491.

(7) Yu, X.; Chen, K.; Guo, S.; Shi, P.; Song, C.; Zhu, J. Direct Access to Cobaltacycles via $\mathrm{C}-\mathrm{H}$ Activation: $\mathrm{N}$-Chloroamide-Enabled Room-Temperature Synthesis of Heterocycles Org. Lett. 2017, 19, 5348.

(8) Yu, X.; Chen, K.; Wang, Q.; Zhang, W.; Zhu, J. Co(III)-Catalyzed N-chloroamidedirected $\mathrm{C}-\mathrm{H}$ activation for 3,4-dihydroisoquinolone synthesis Org. Chem. Front. 2018, 5, 994.

(9) Ye, B.; Cramer, N. Chiral Cyclopentadienyl Ligands as Stereocontrolling Element in Asymmetric C-H Functionalization Science 2012, 338, 504.

(10) Grunewald, G. L.; Romero, F. A.; Chieu, A. D.; Fincham, K. J.; Bhat, S. R.; Criscione, K. R. Exploring the active site of phenylethanolamine $\mathrm{N}$-methyltransferase: $3-$ alkyl-7-substituted-1,2,3,4-tetrahydroisoquinoline inhibitors Biorg. Med. Chem. 2005, 13, 1261.

(11) Trifonova, E. A.; Ankudinov, N. M.; Kozlov, M. V.; Sharipov, M. Y.; Nelyubina, Y. V.; Perekalin, D. S. Rhodium(III) Complex with a Bulky Cyclopentadienyl Ligand as a Catalyst for Regioselective Synthesis of Dihydroisoquinolones through $\mathrm{C}-\mathrm{H}$ Activation of Arylhydroxamic Acids Chem. - Eur. J. 2018, 24, 16570.

(12) Smith, A. B.; Cantin, L.-D.; Pasternak, A.; Guise-Zawacki, L.; Yao, W.; Charnley, A. K.; Barbosa, J.; Sprengeler, P. A.; Hirschmann, R.; Munshi, S.; Olsen, D. B.; Schleif, W. A.; Kuo, L. C. Design, Synthesis, and Biological Evaluation of Monopyrrolinone-Based HIV-1 Protease Inhibitors J. Med. Chem. 2003, 46, 1831.

(13) Heinrich, M. R.; Blank, O.; Wölfel, S. Reductive Carbodiazenylation of Nonactivated Olefins via Aryl Diazonium Salts Org. Lett. 2006, 8, 3323.

(14) Trifonova, E. A.; Ankudinov, N. M.; Mikhaylov, A. A.; Chusov, D. A.; Nelyubina, Y. V.; Perekalin, D. S. A Planar-Chiral Rhodium(III) Catalyst with a Sterically Demanding Cyclopentadienyl Ligand and Its Application in the Enantioselective Synthesis of Dihydroisoquinolones Angew. Chem., Int. Ed. 2018, 57, 7714.

(15) Poater, A.; Ragone, F.; Giudice, S.; Costabile, C.; Dorta, R.; Nolan, S. P.; Cavallo, $\mathrm{L}$. Thermodynamics of $\mathrm{N}$-Heterocyclic Carbene Dimerization: The Balance of Sterics and Electronics Organometallics 2008, 27, 2679. 


\section{Spectra}




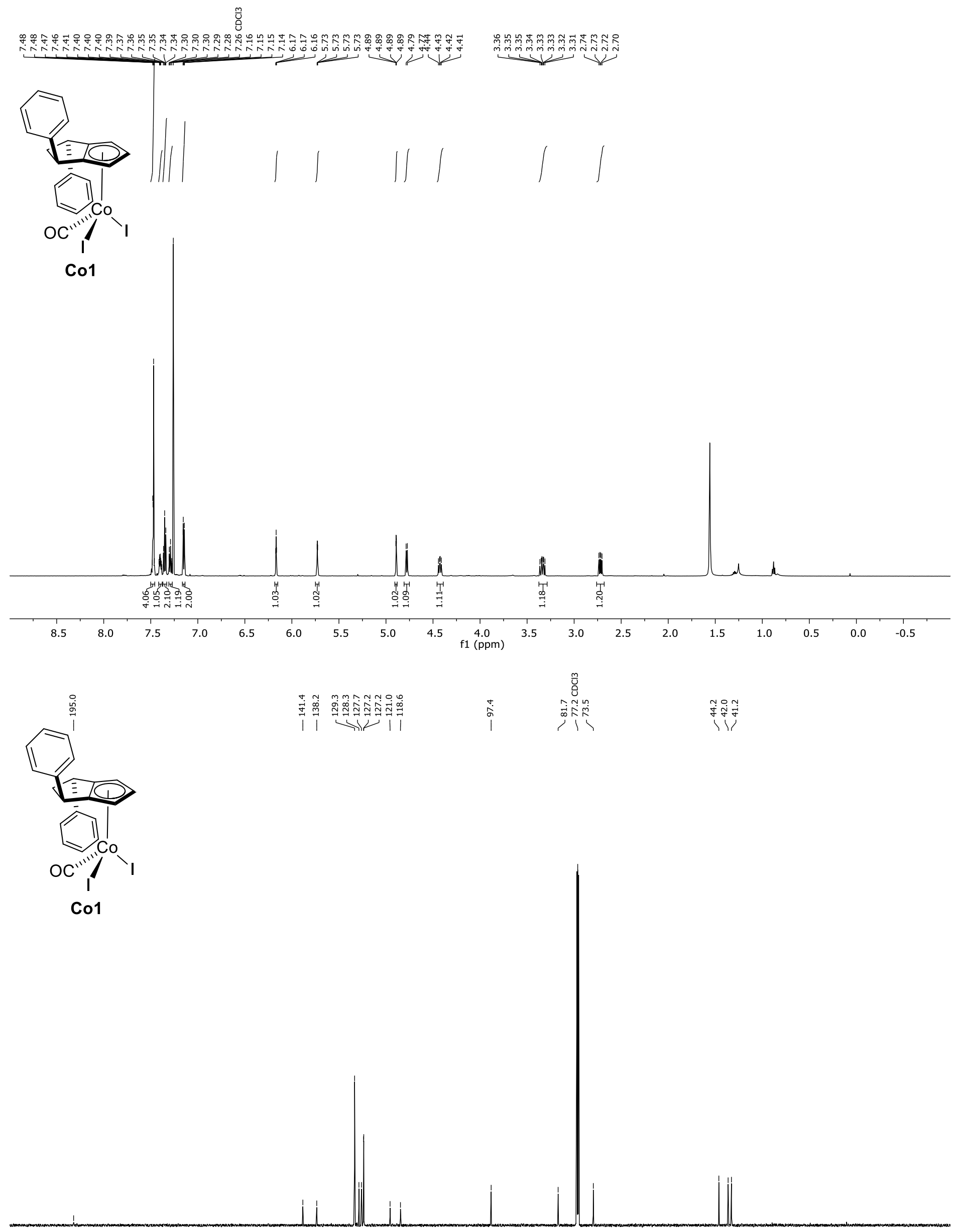



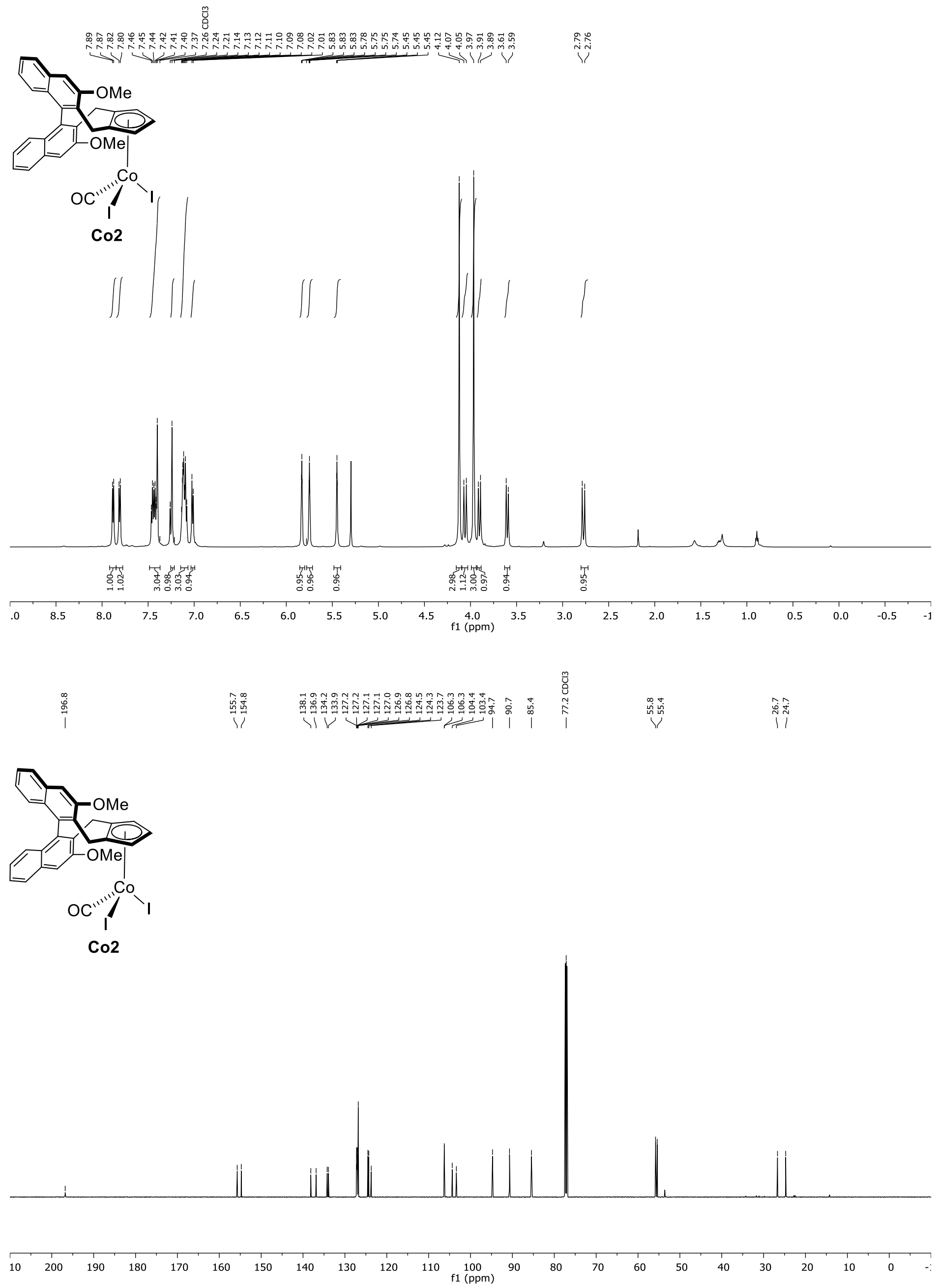

S55 

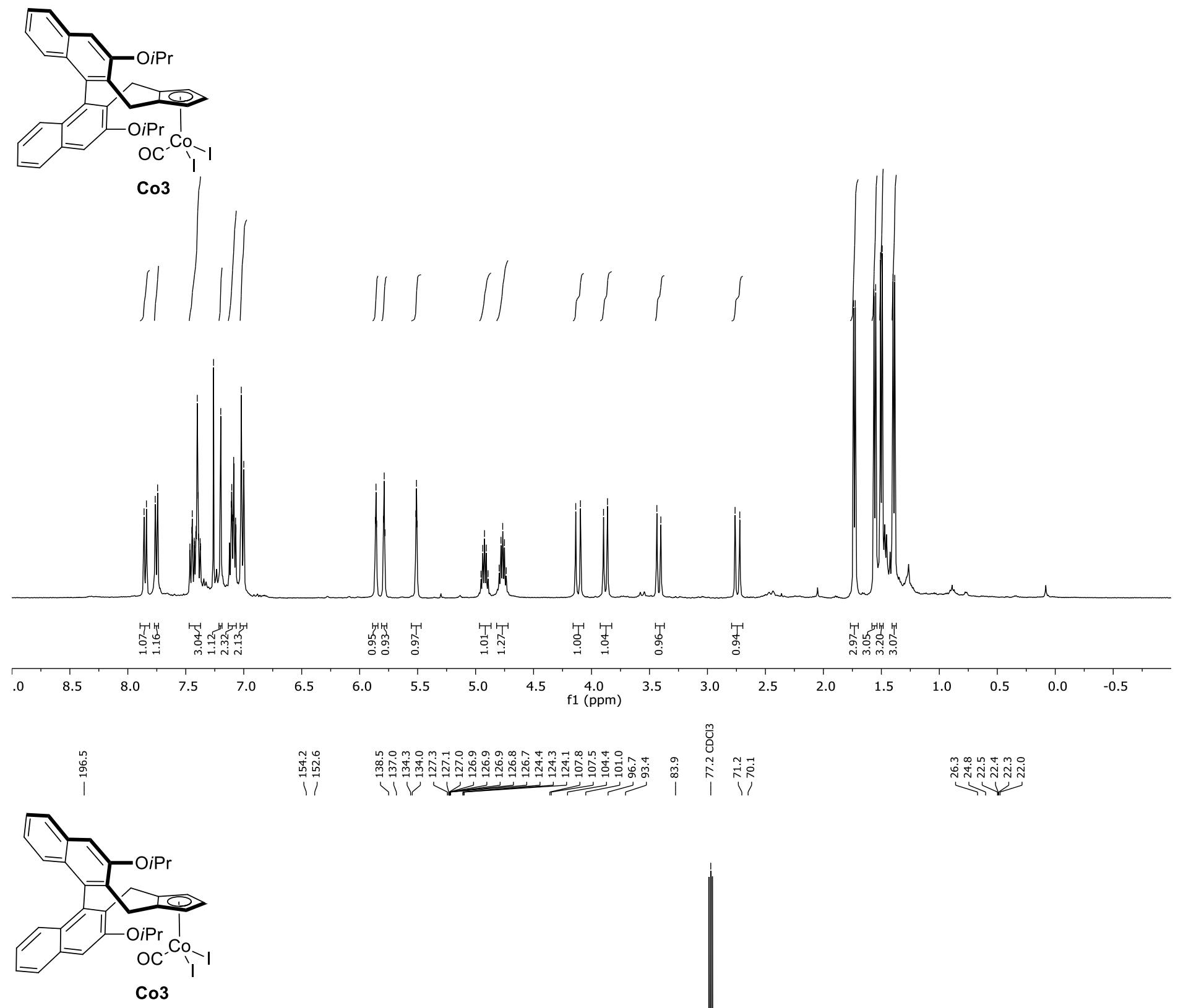

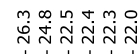

4

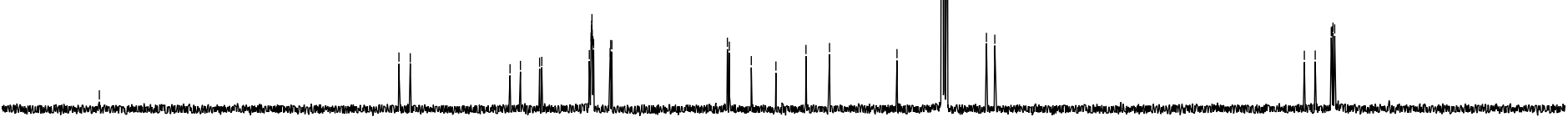



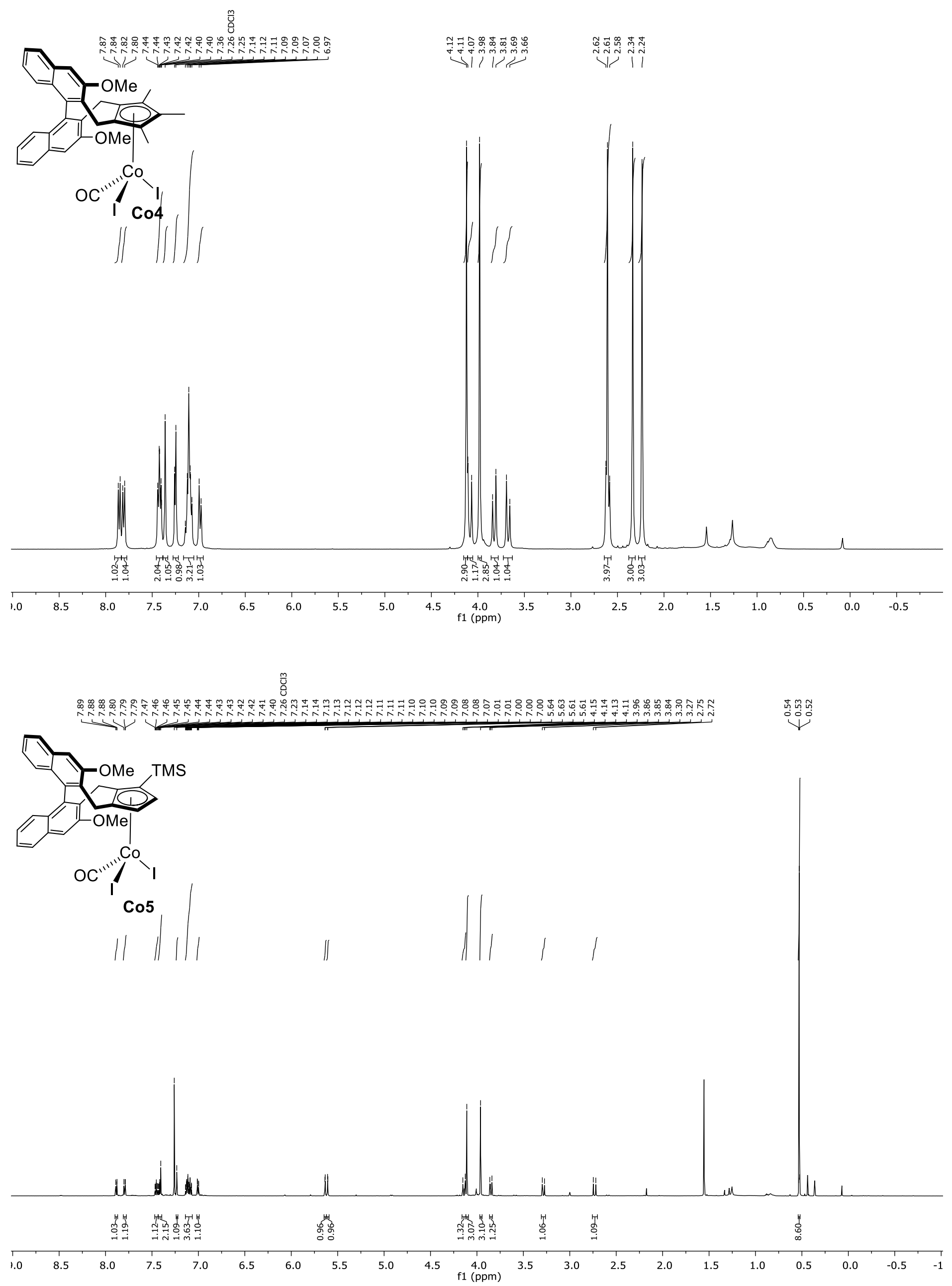

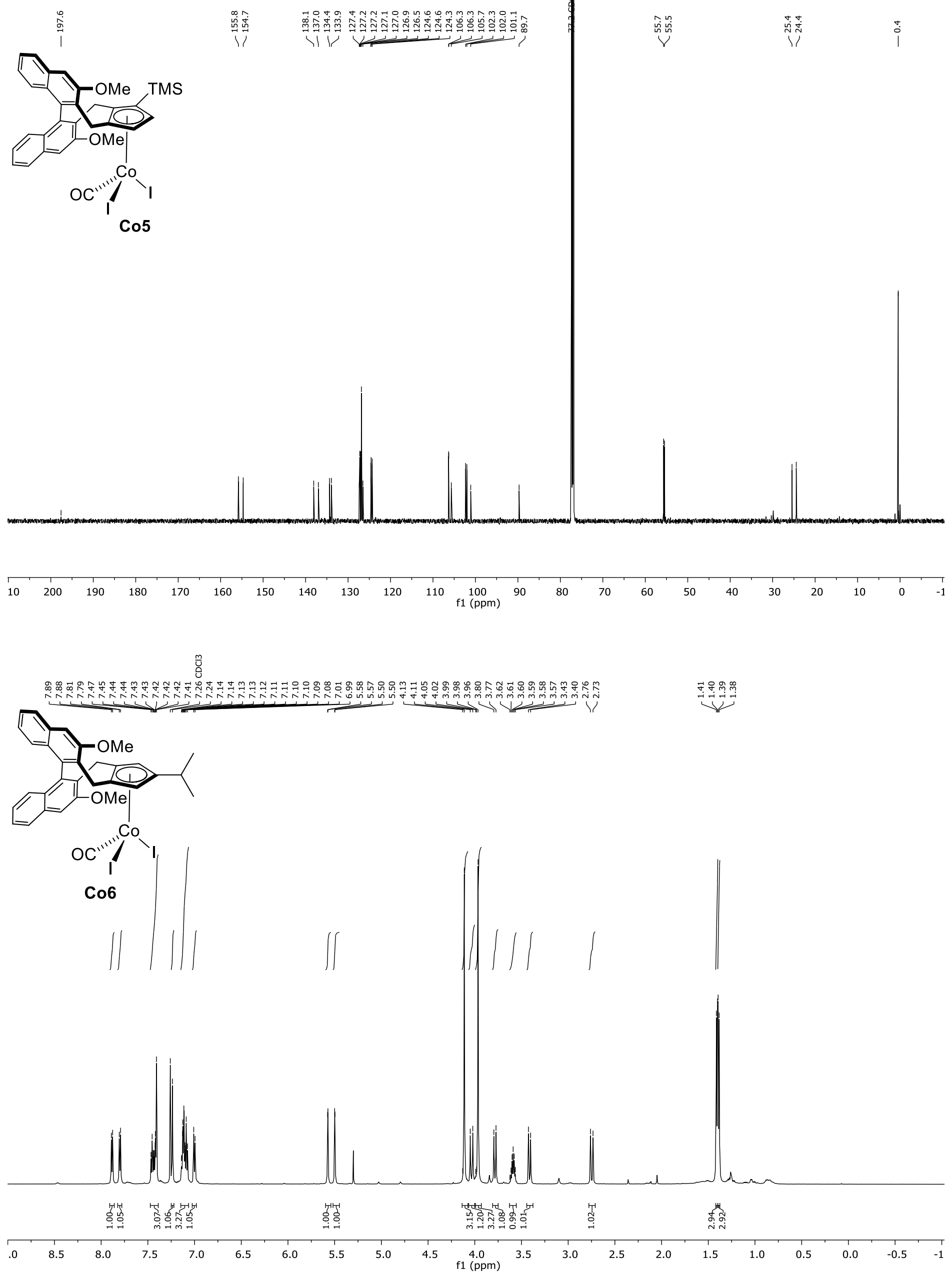

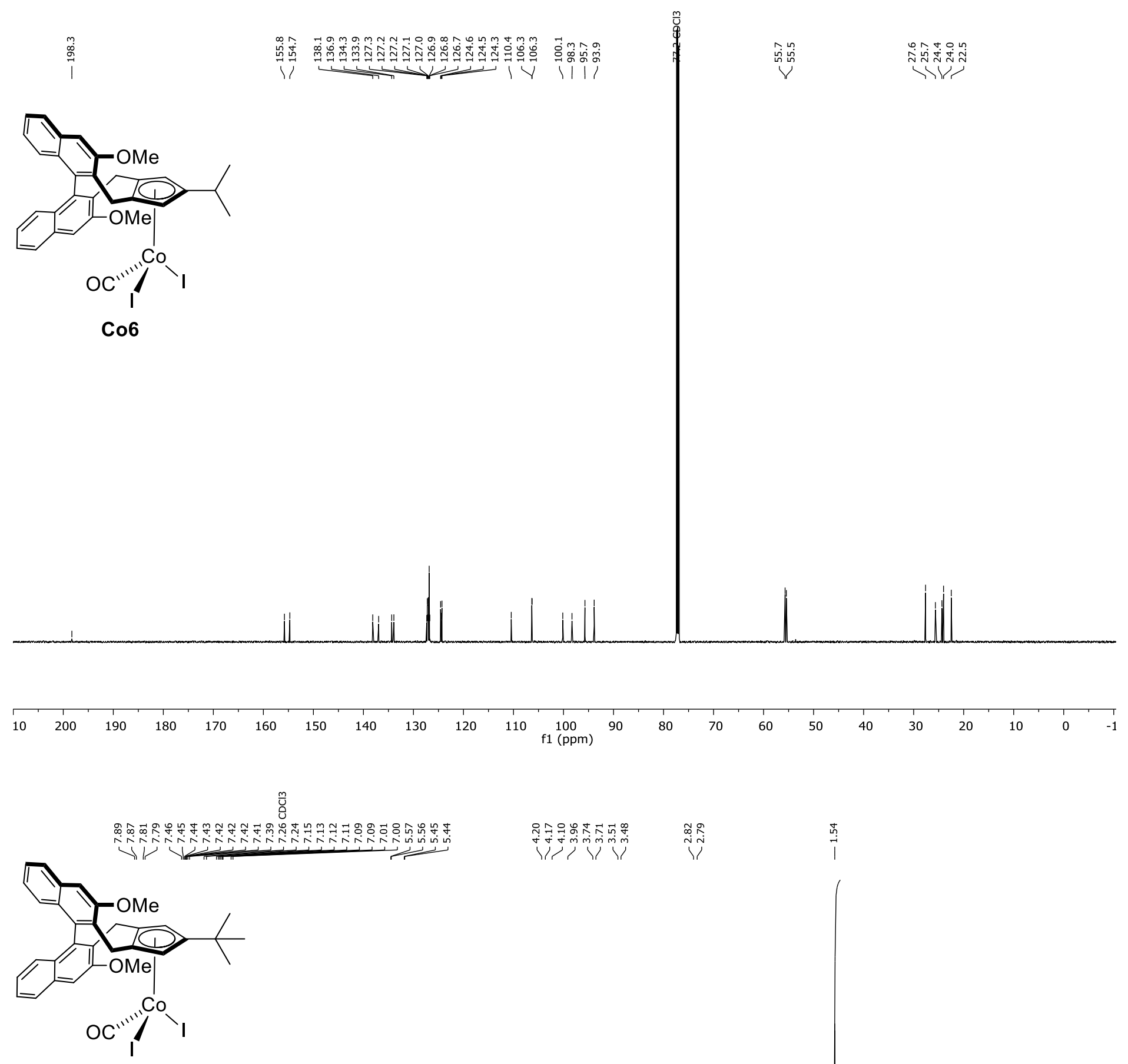

पू

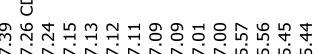
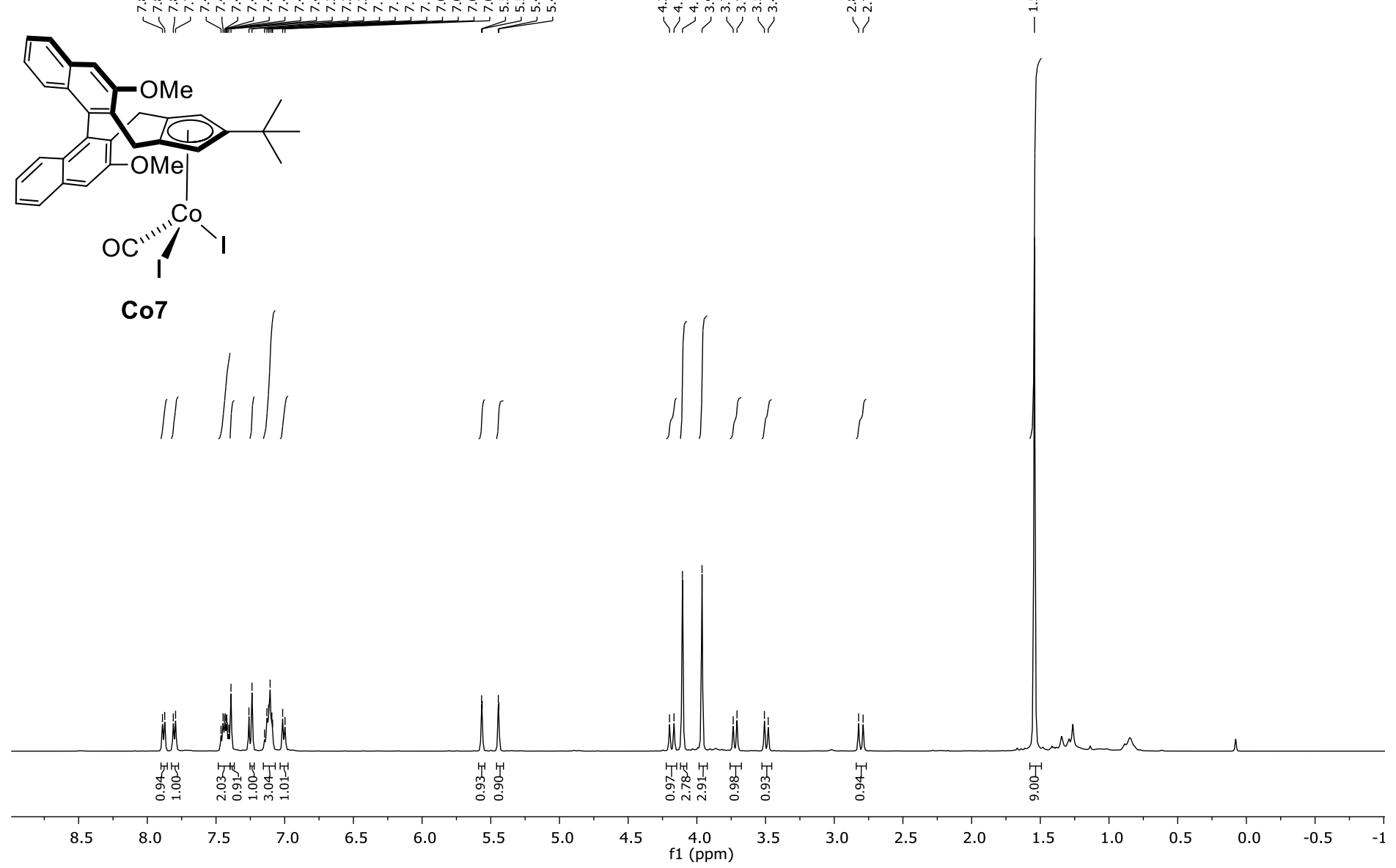


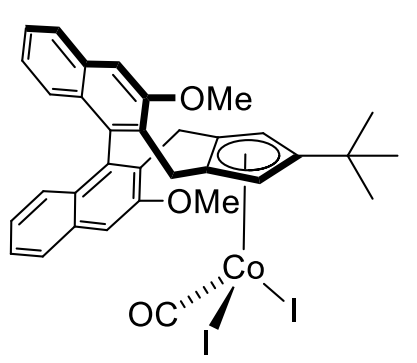

Co7

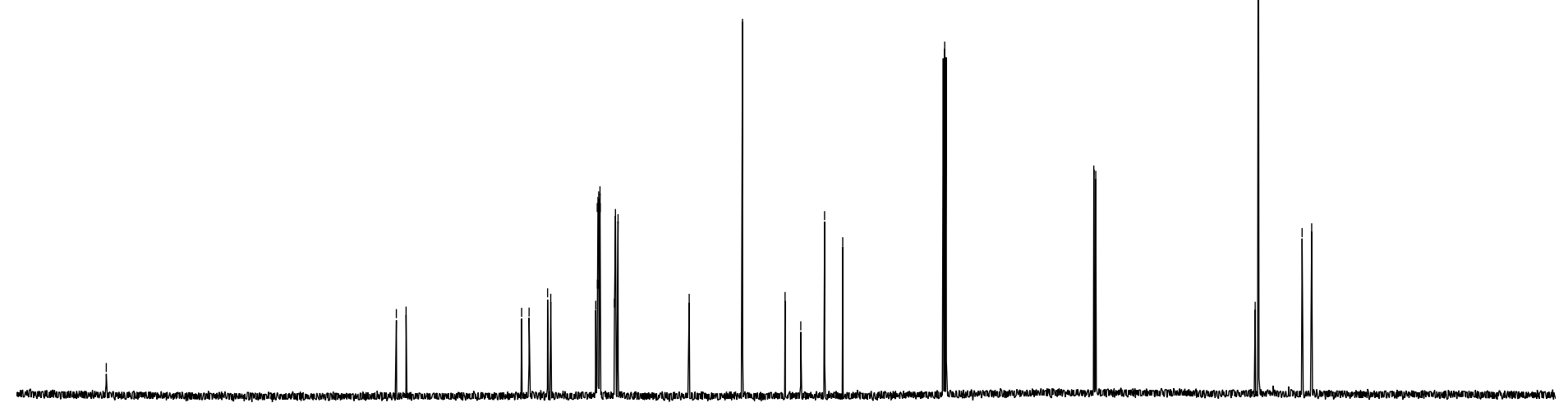

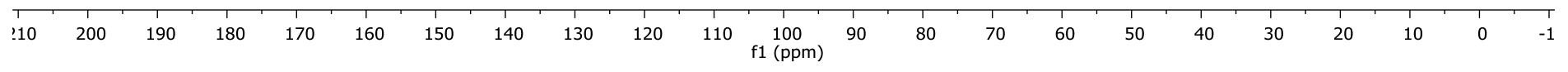

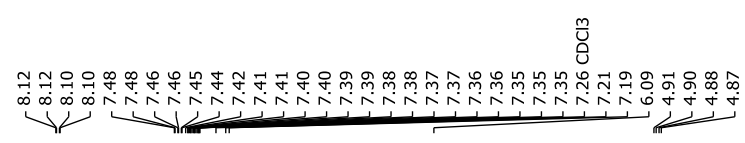

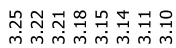

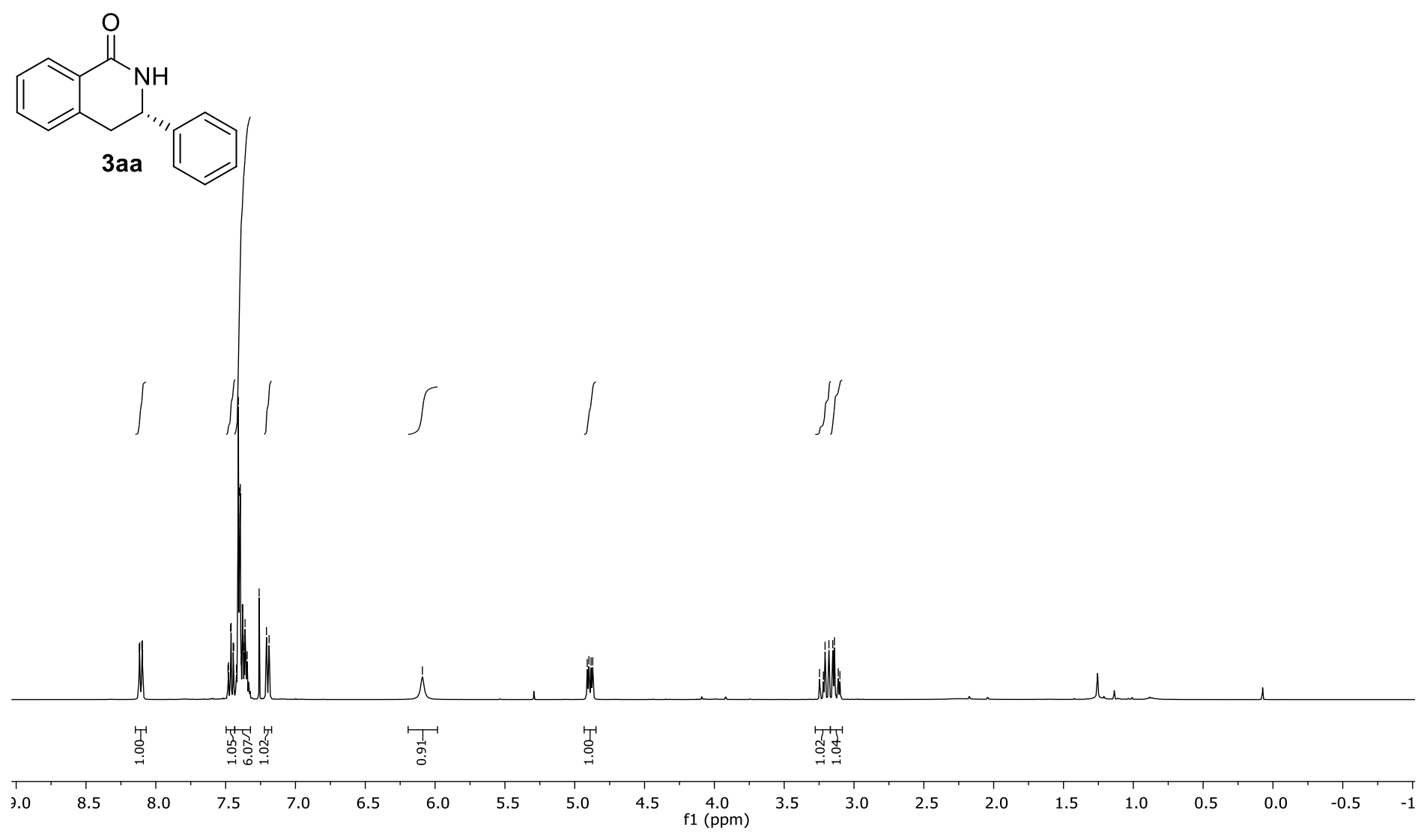



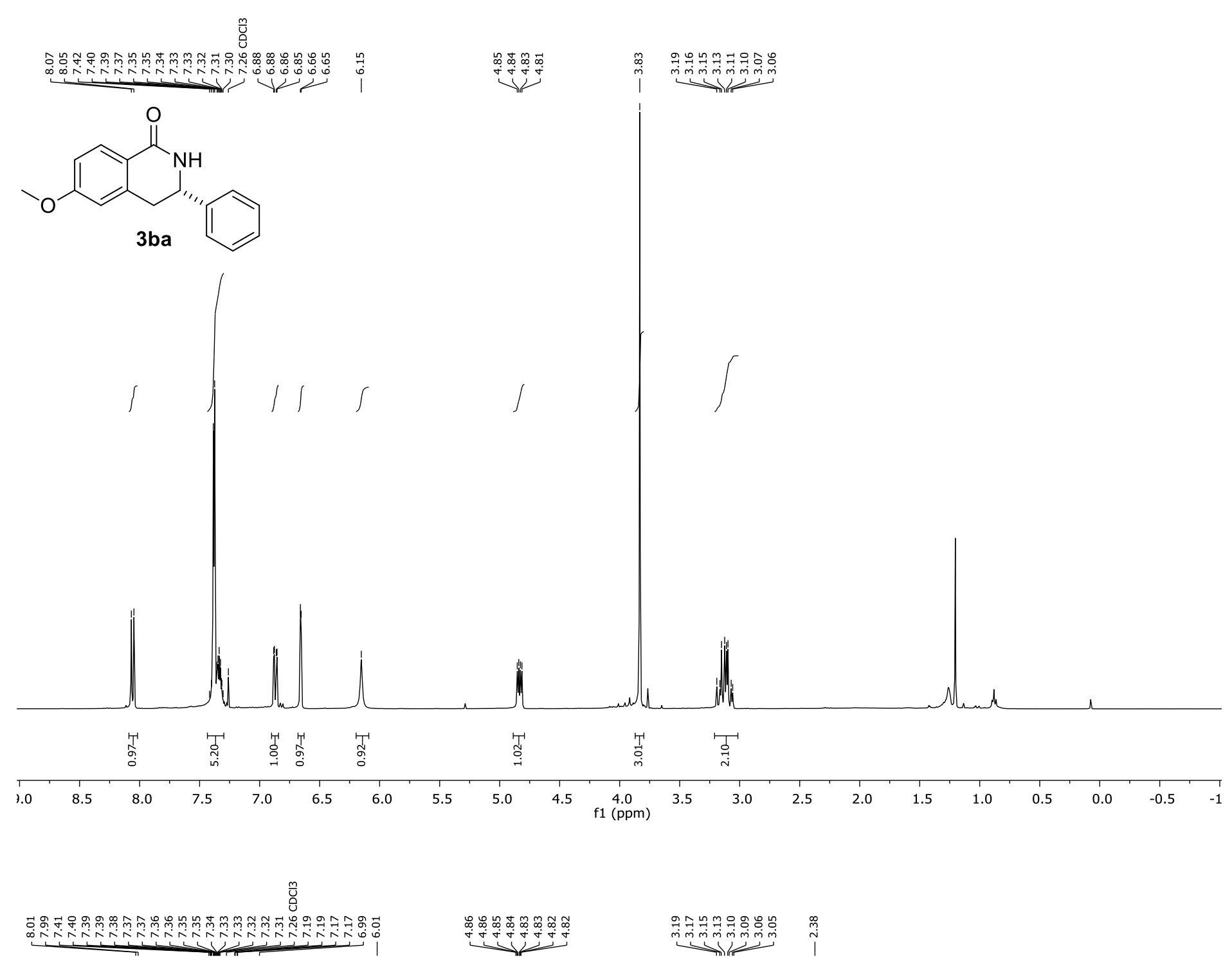<smiles>Cc1ccc2c(c1)C[C@@H](c1ccccc1)NC2=O</smiles>

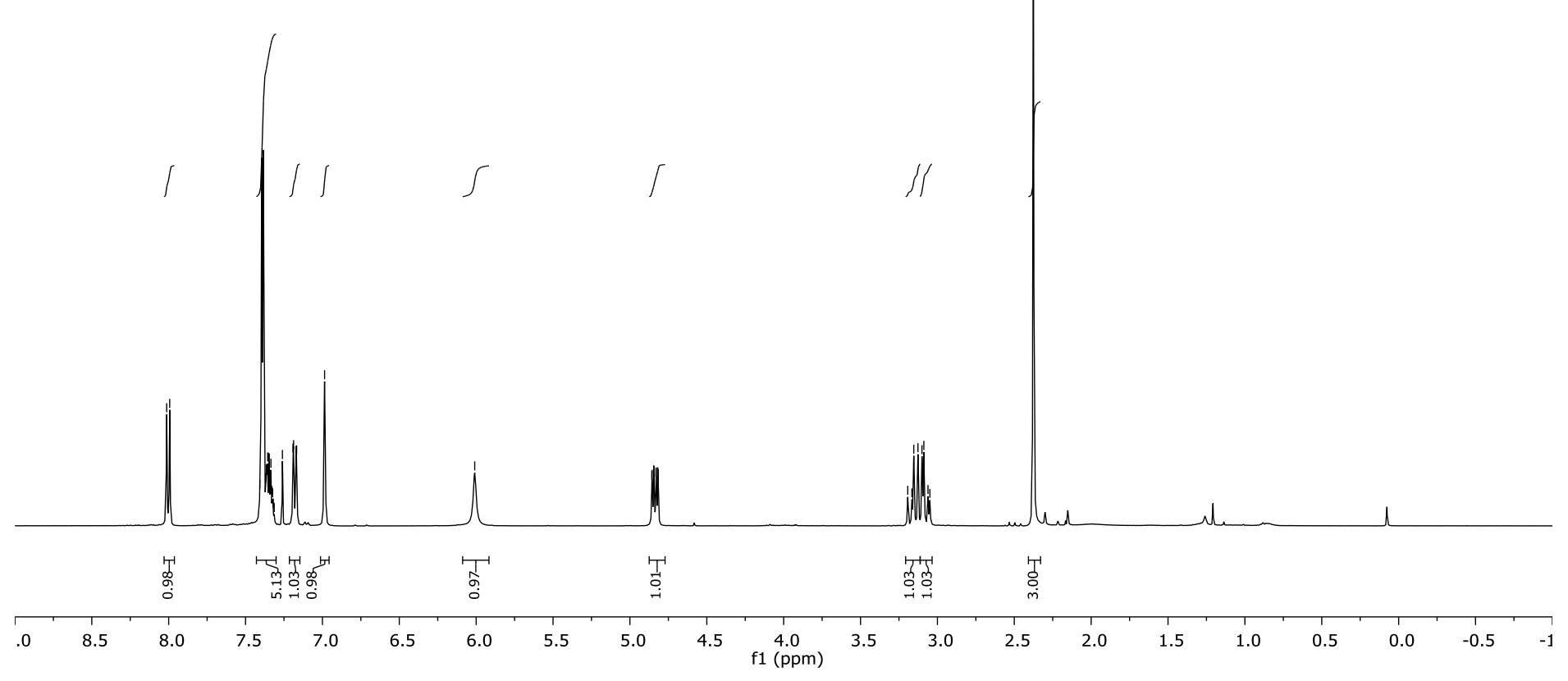




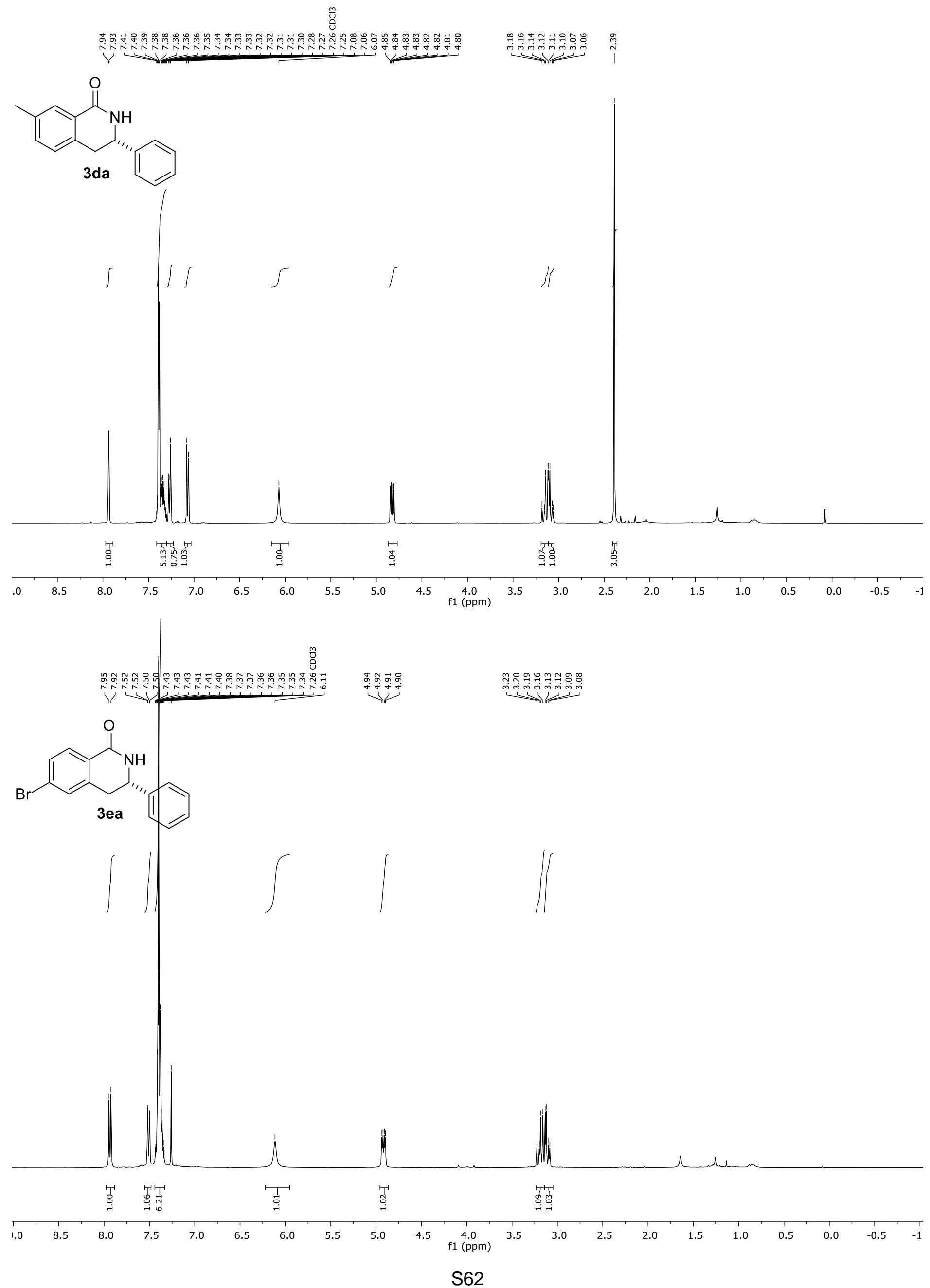



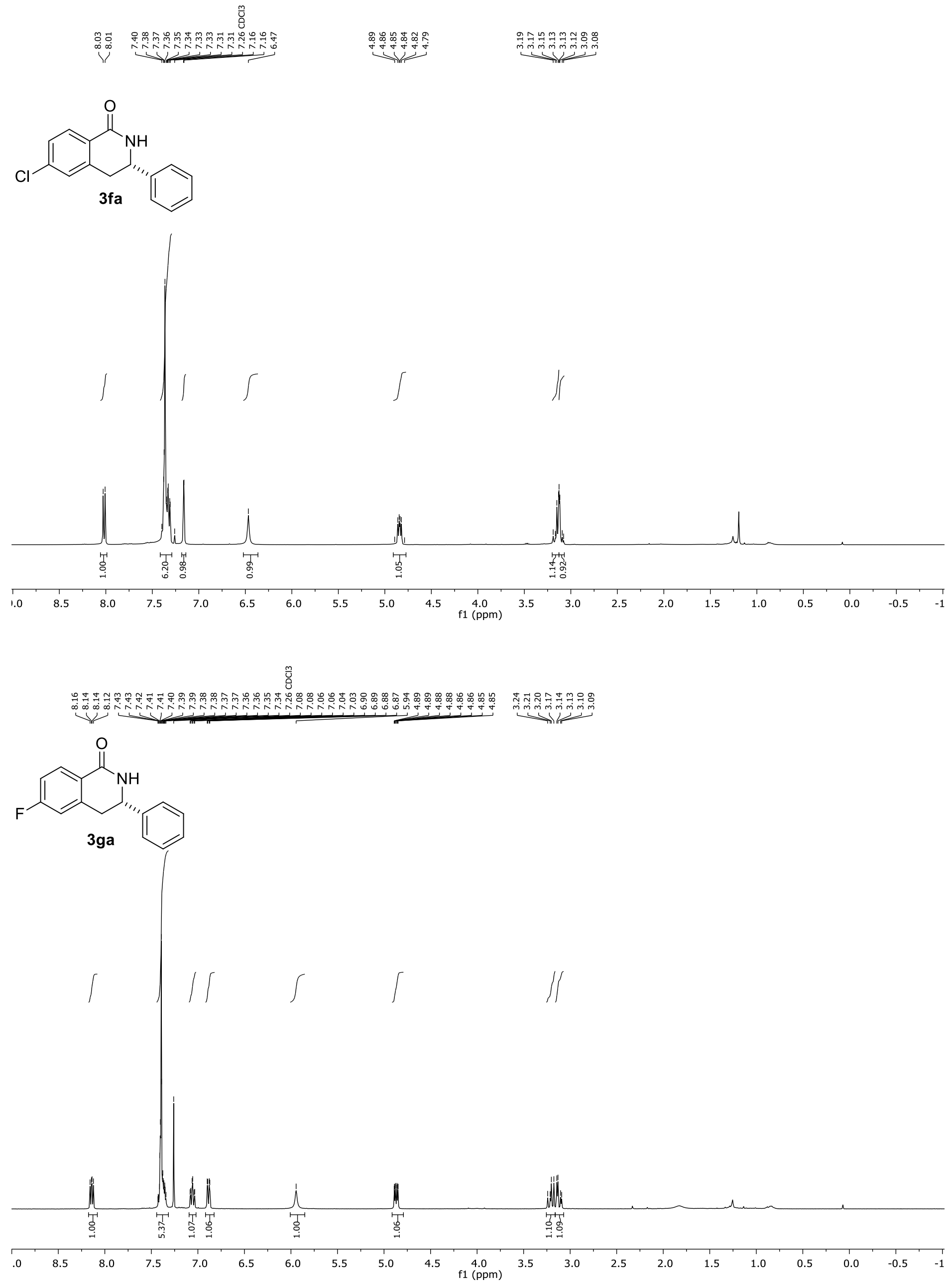
<smiles>O=C1N[C@@H](c2ccccc2)Cc2cc(F)ccc21</smiles>
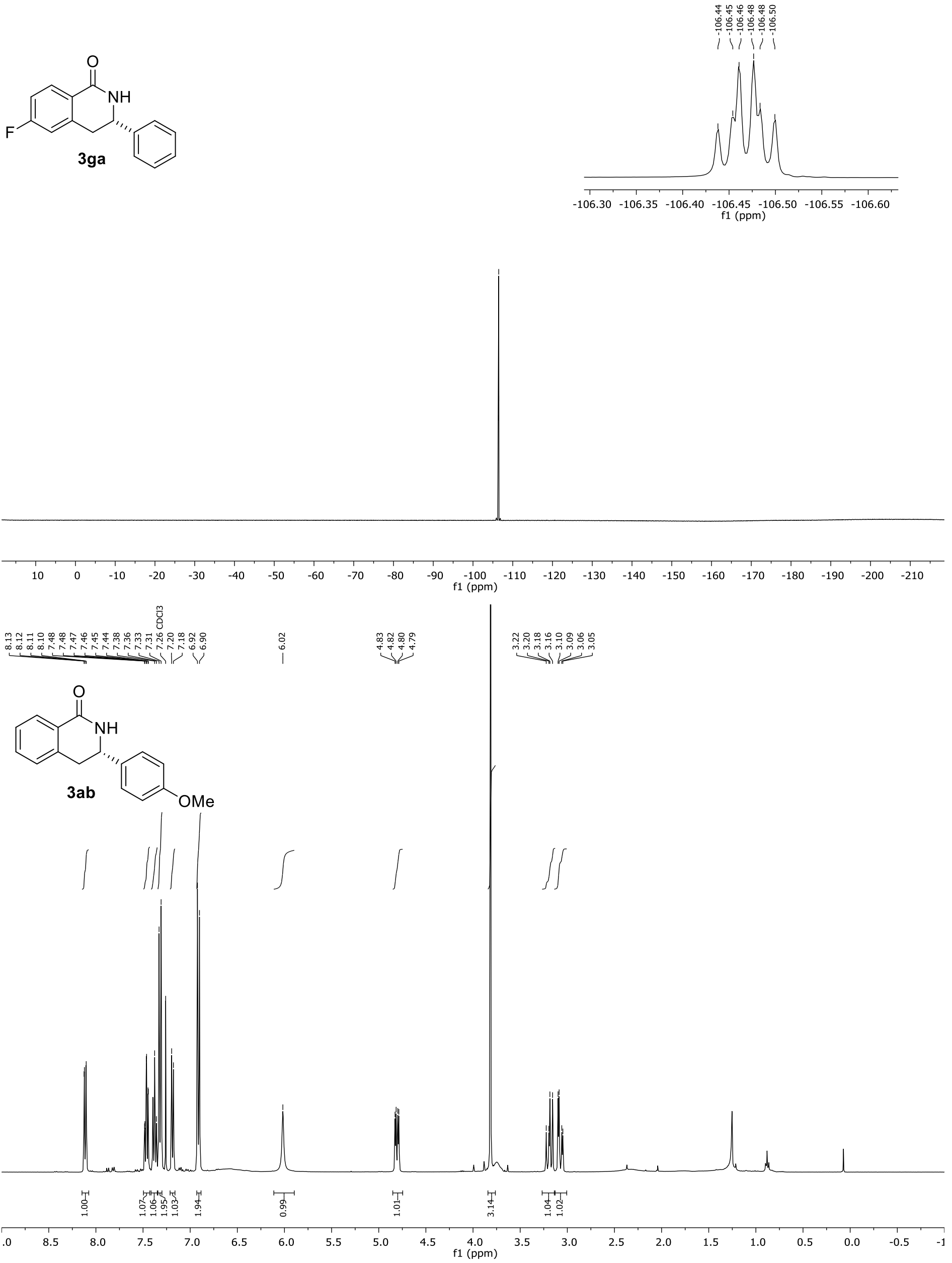
<smiles>CC(C)(C)c1ccc([C@@H]2Cc3ccccc3C(=O)N2)cc1</smiles><smiles>CC=CC</smiles>
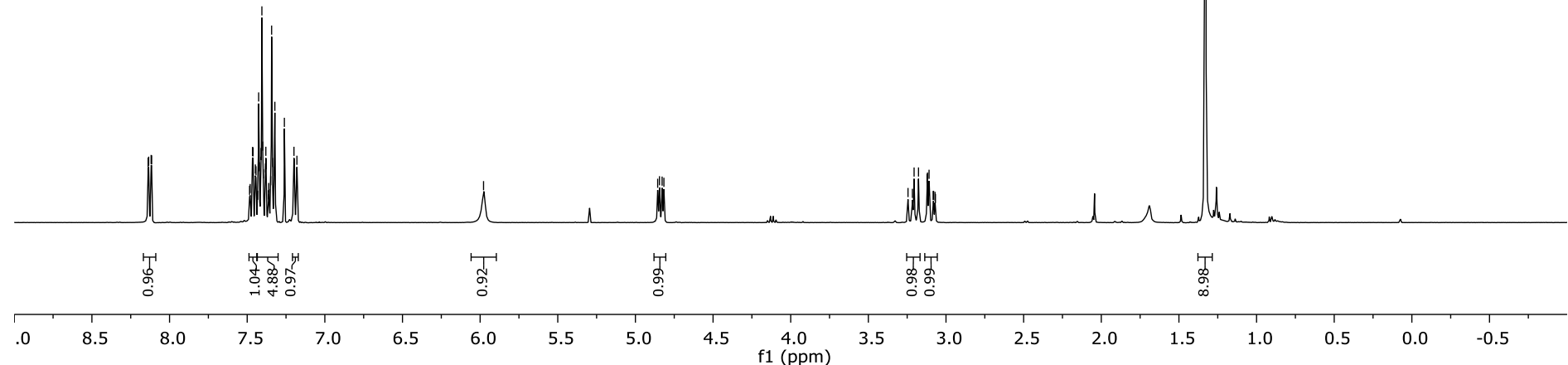

莭

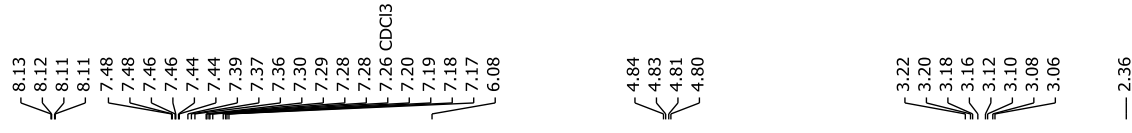<smiles>Cc1ccc([C@@H]2Cc3ccccc3C(=O)N2)cc1</smiles><smiles>C#CCCC</smiles><smiles>C1CCCC1</smiles>
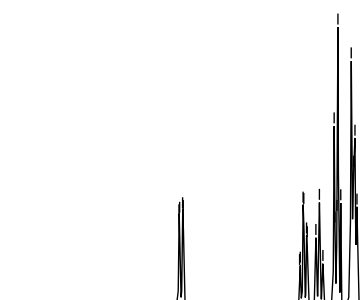<smiles>C1=CC=C1</smiles>
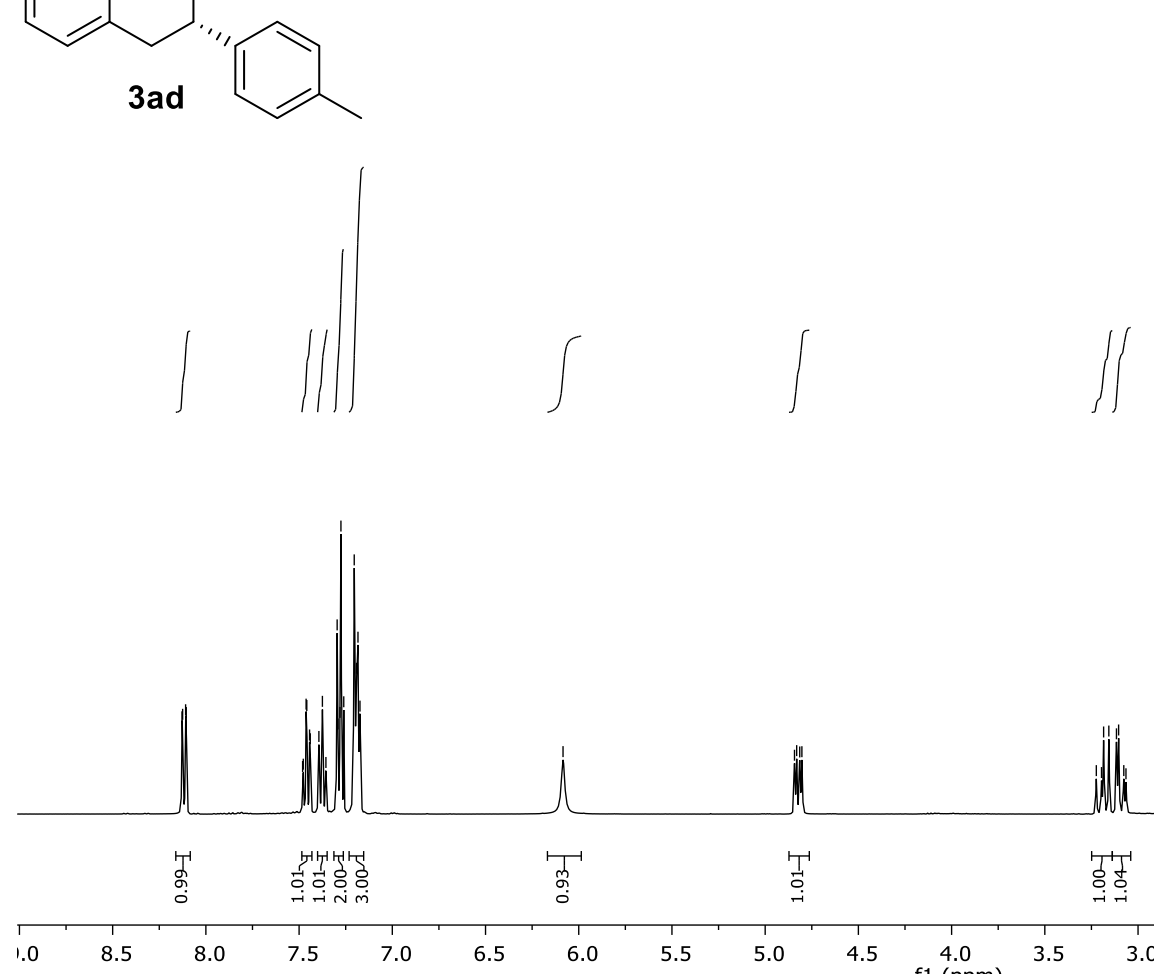

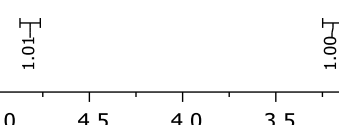

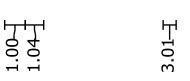




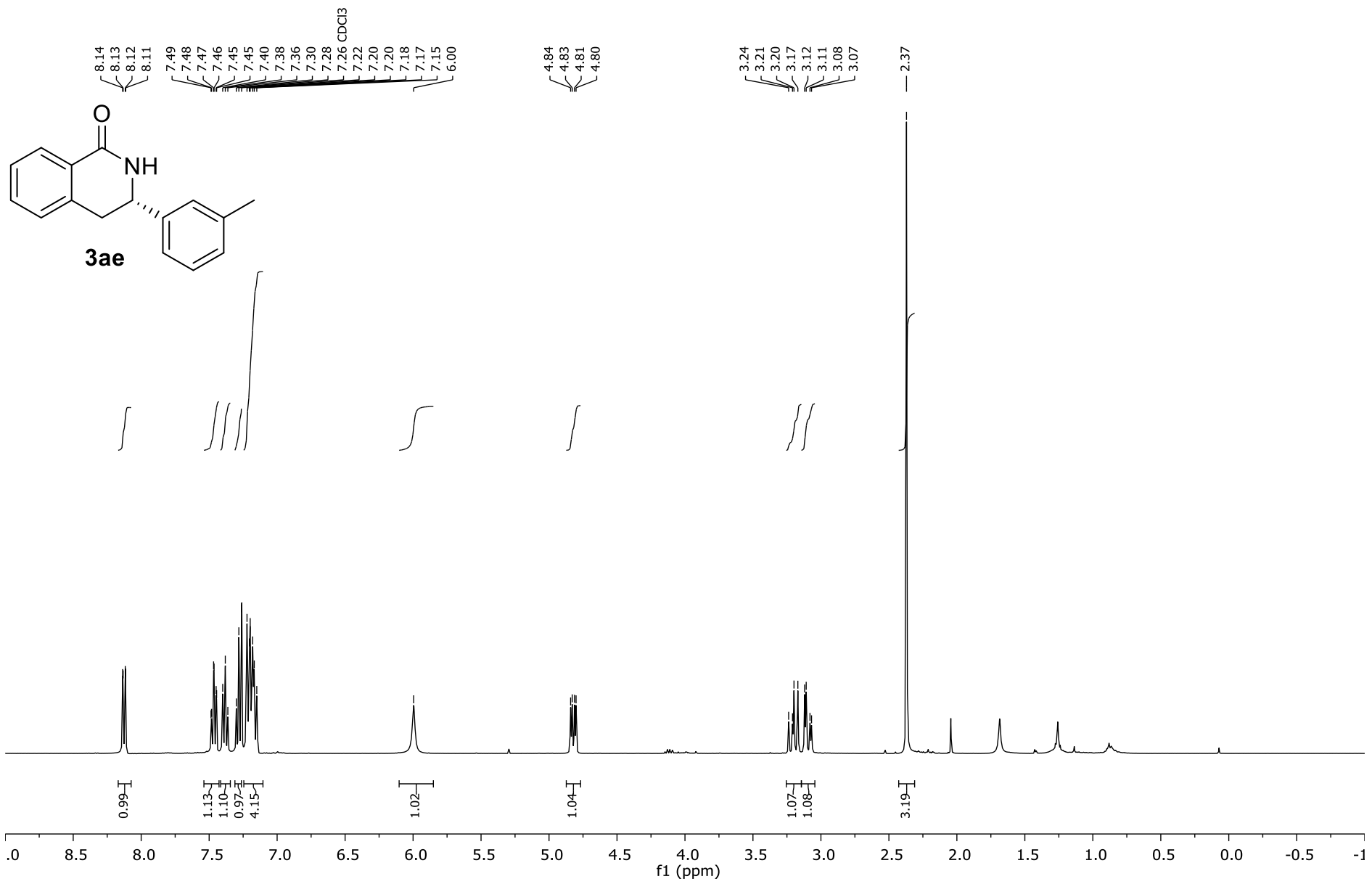

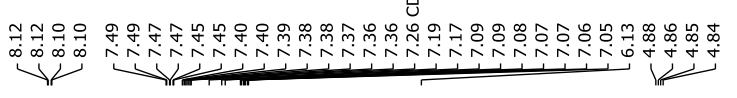

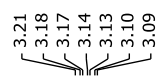
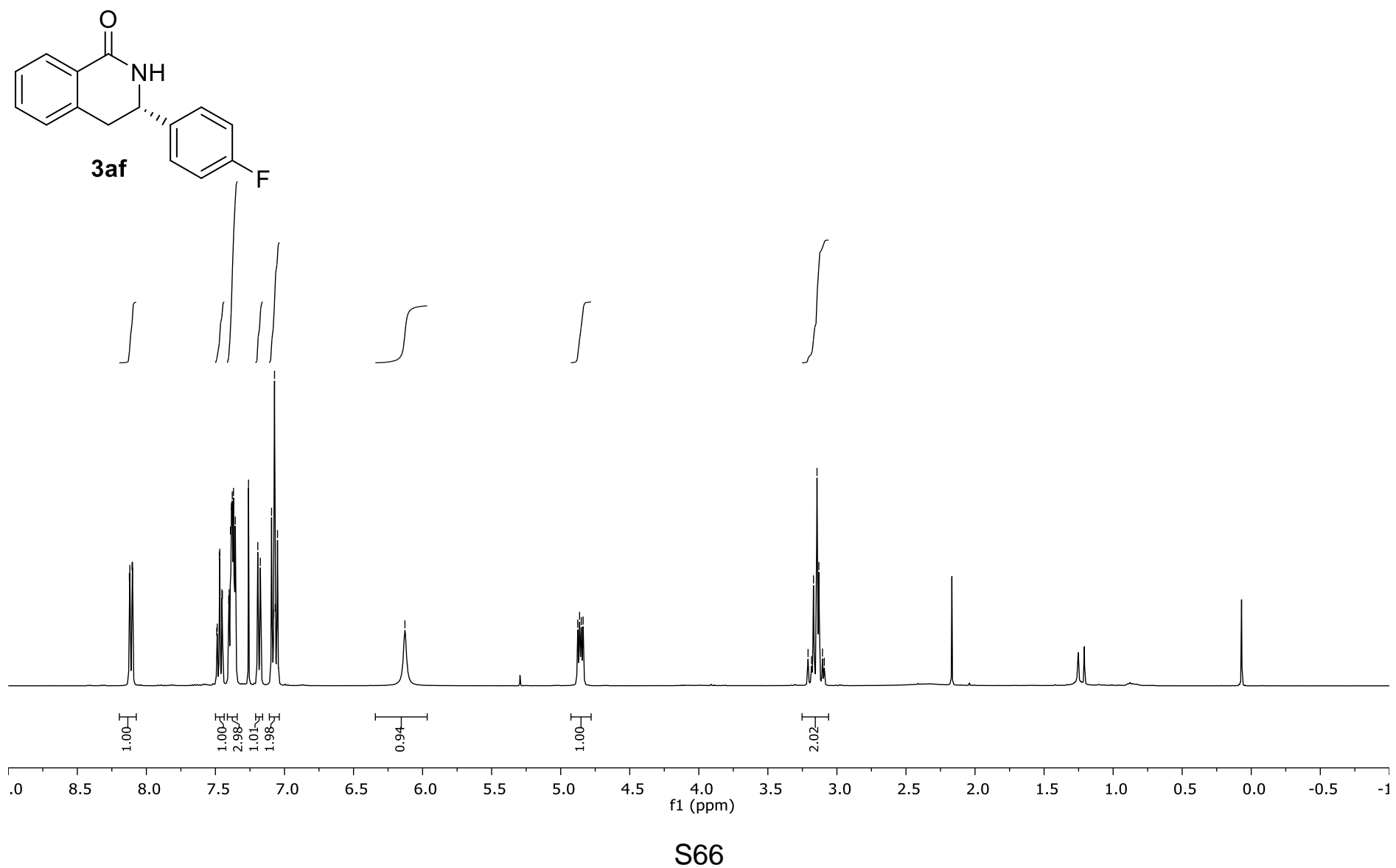
<smiles>O=C1N[C@H](c2ccc(F)cc2)Cc2ccccc21</smiles>

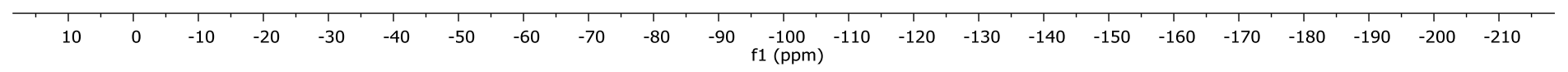
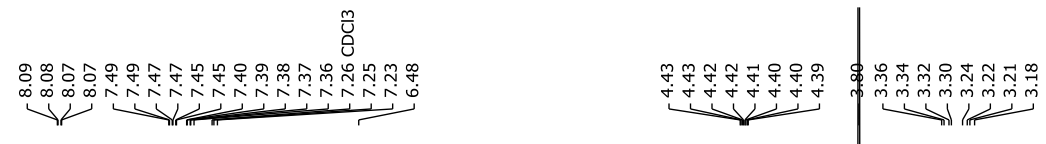<smiles>COC(=O)[C@H]1Cc2ccccc2C(=O)N1</smiles>

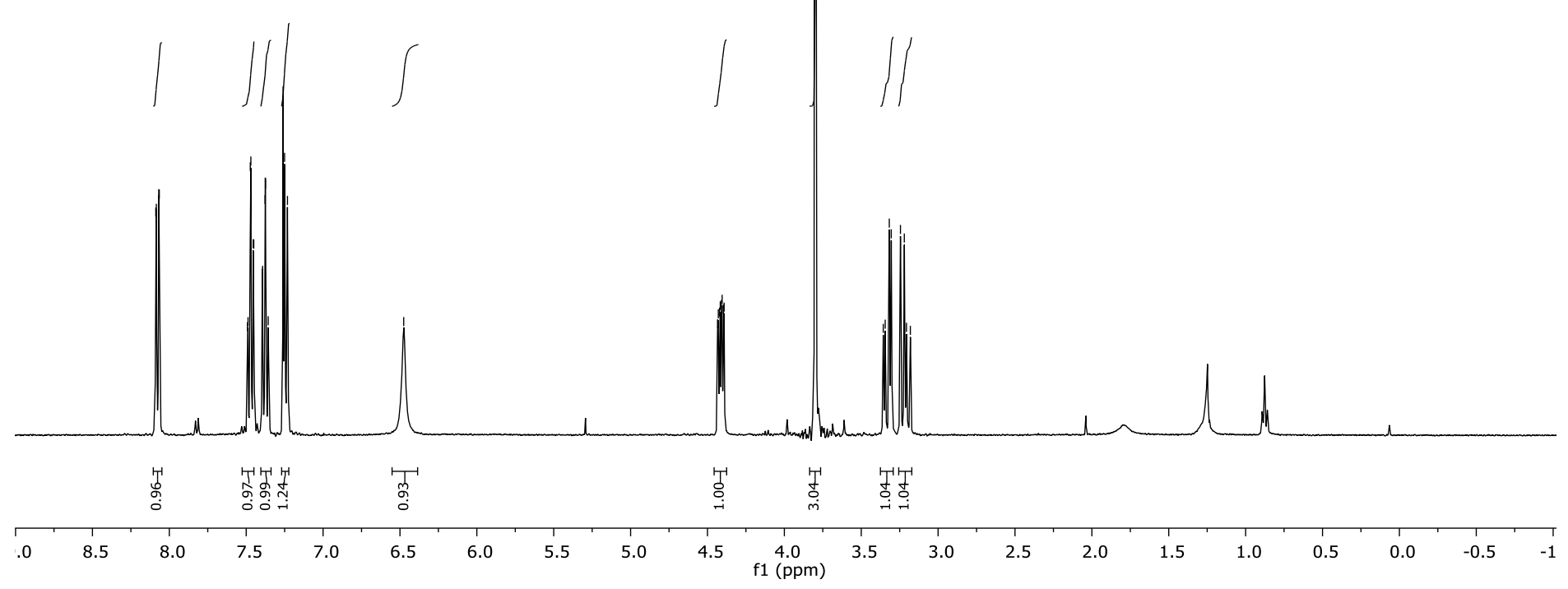




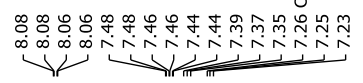

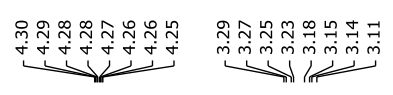<smiles>CC(C)(C)OC(=O)[C@@H]1Cc2ccccc2C(=O)N1</smiles>
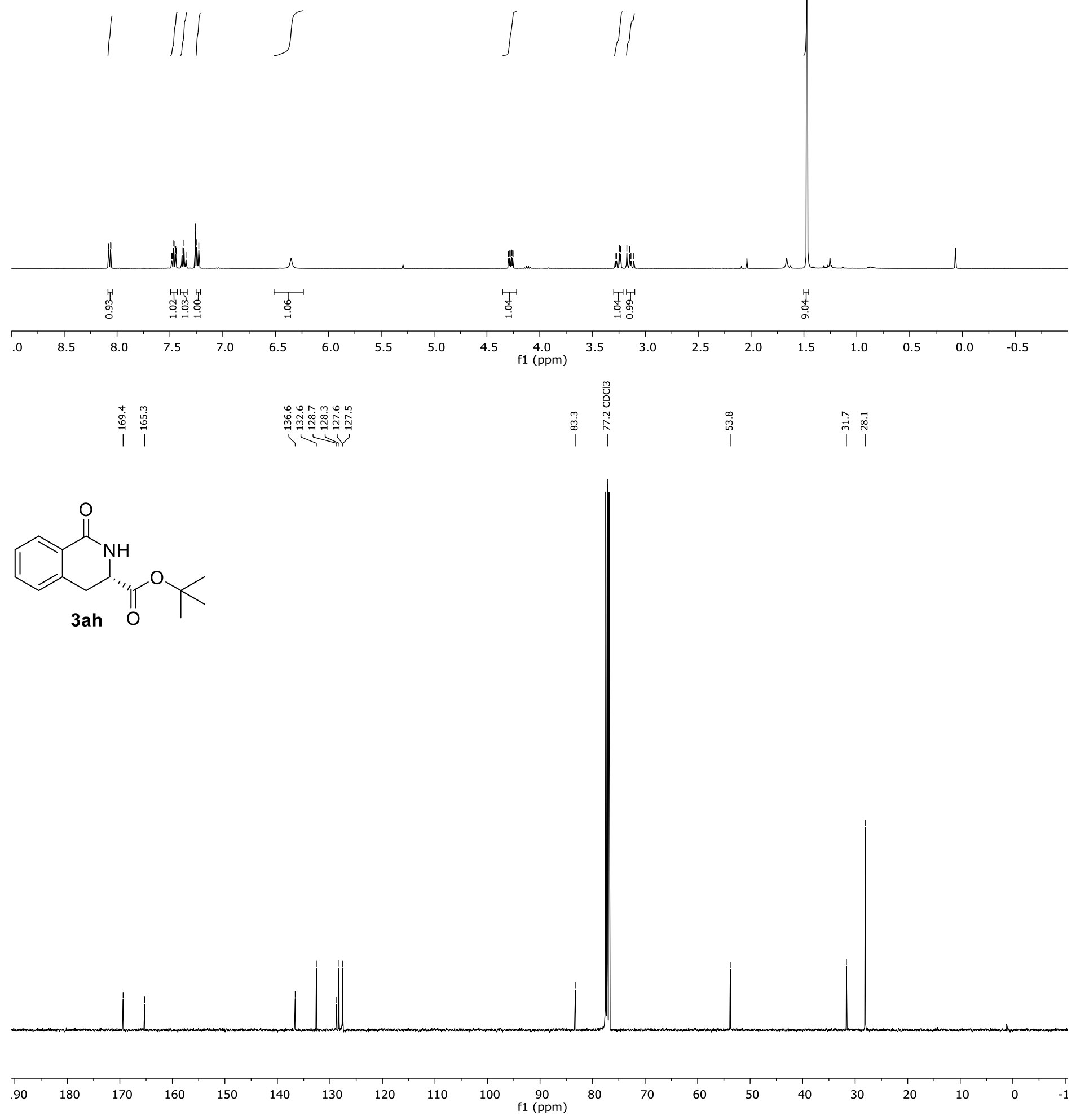

S68 


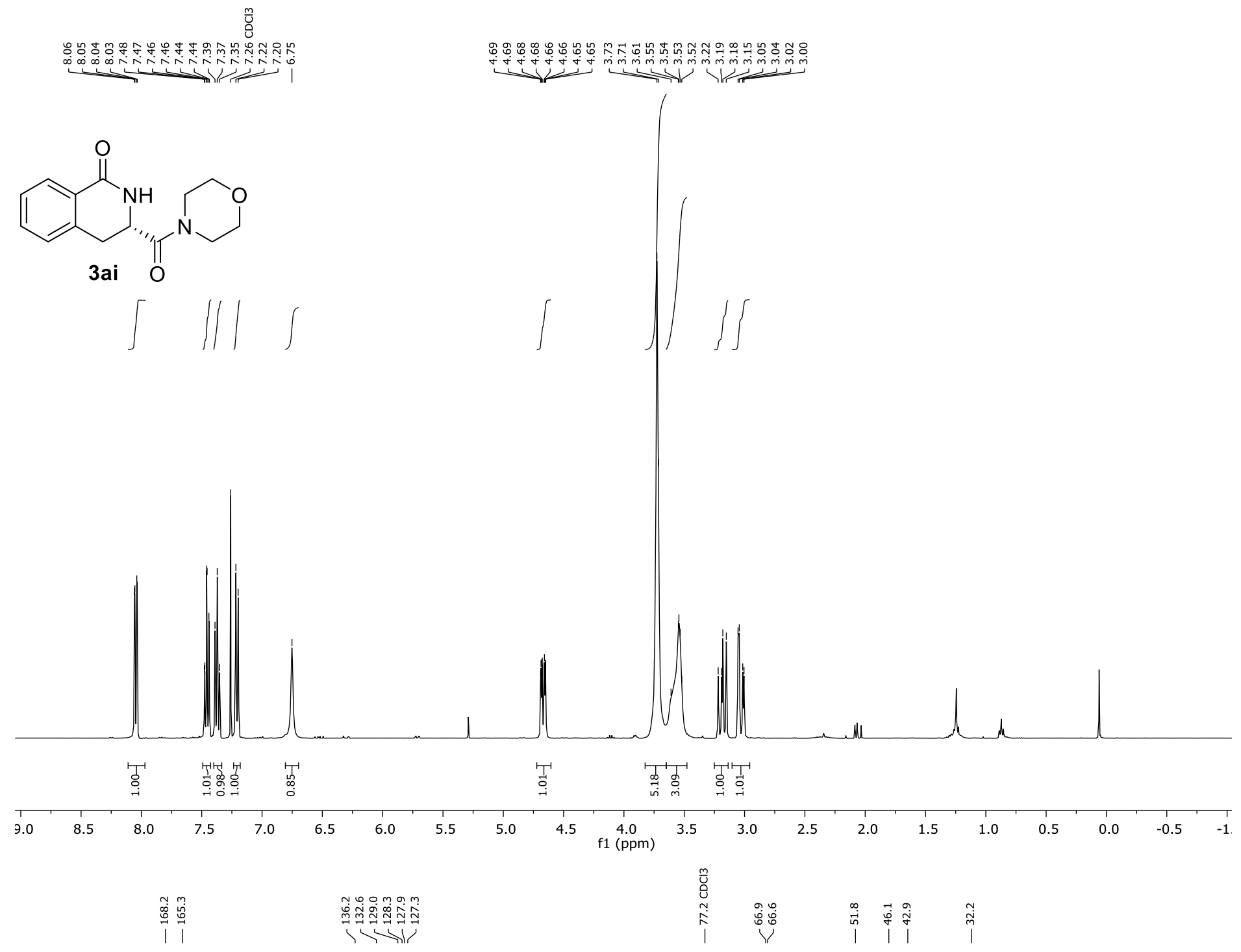<smiles>O=C1N[C@H](C(=O)N2CCOCC2)Cc2ccccc21</smiles>

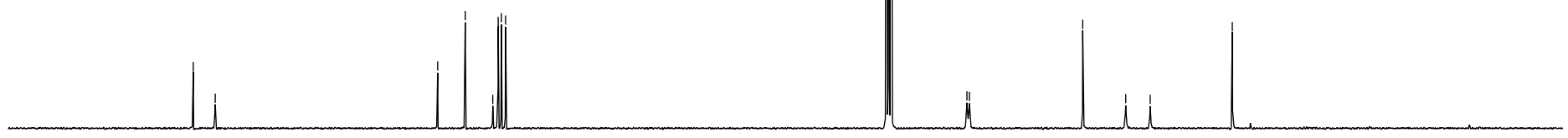

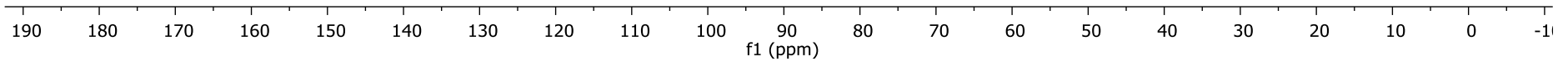




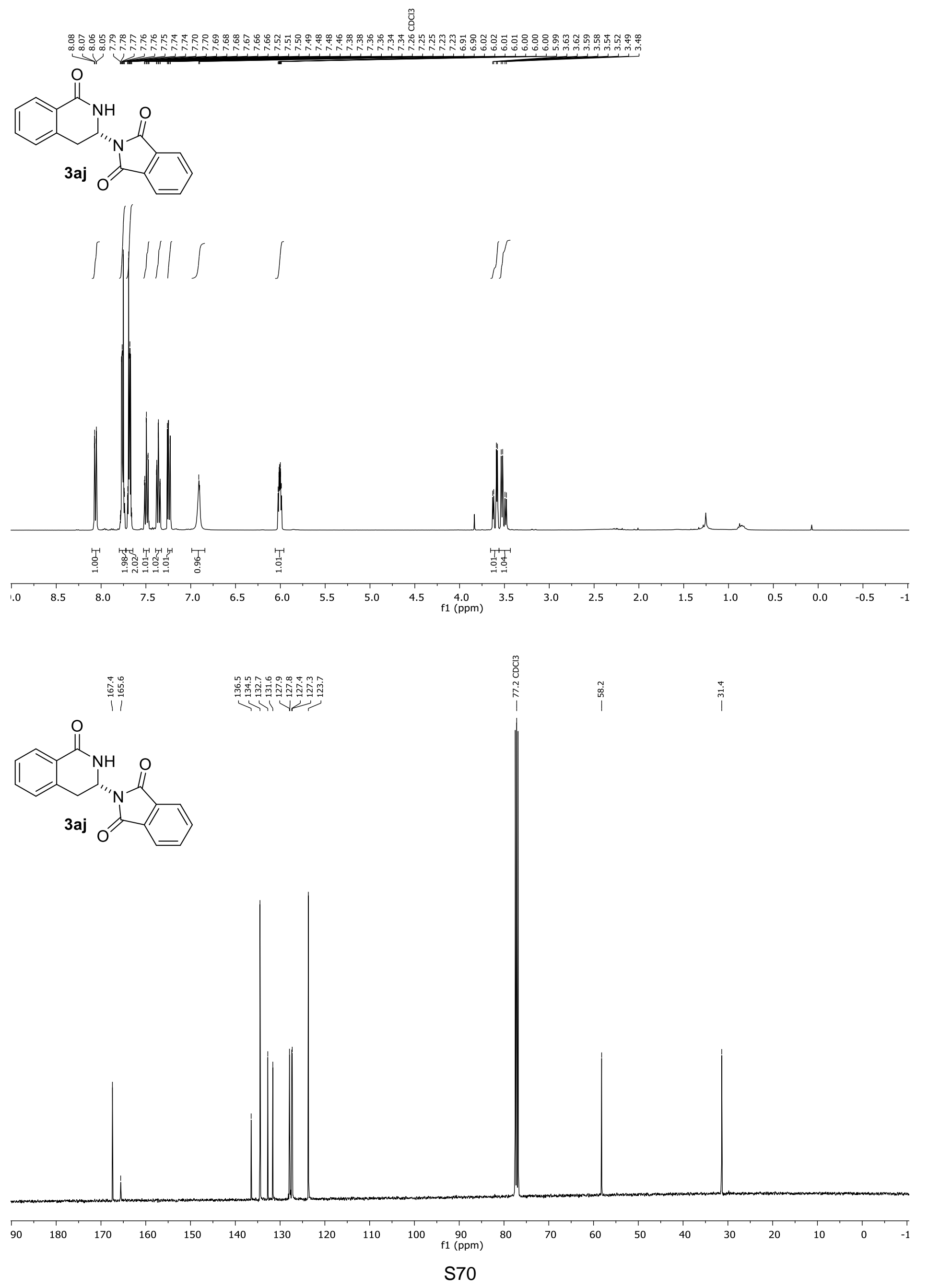




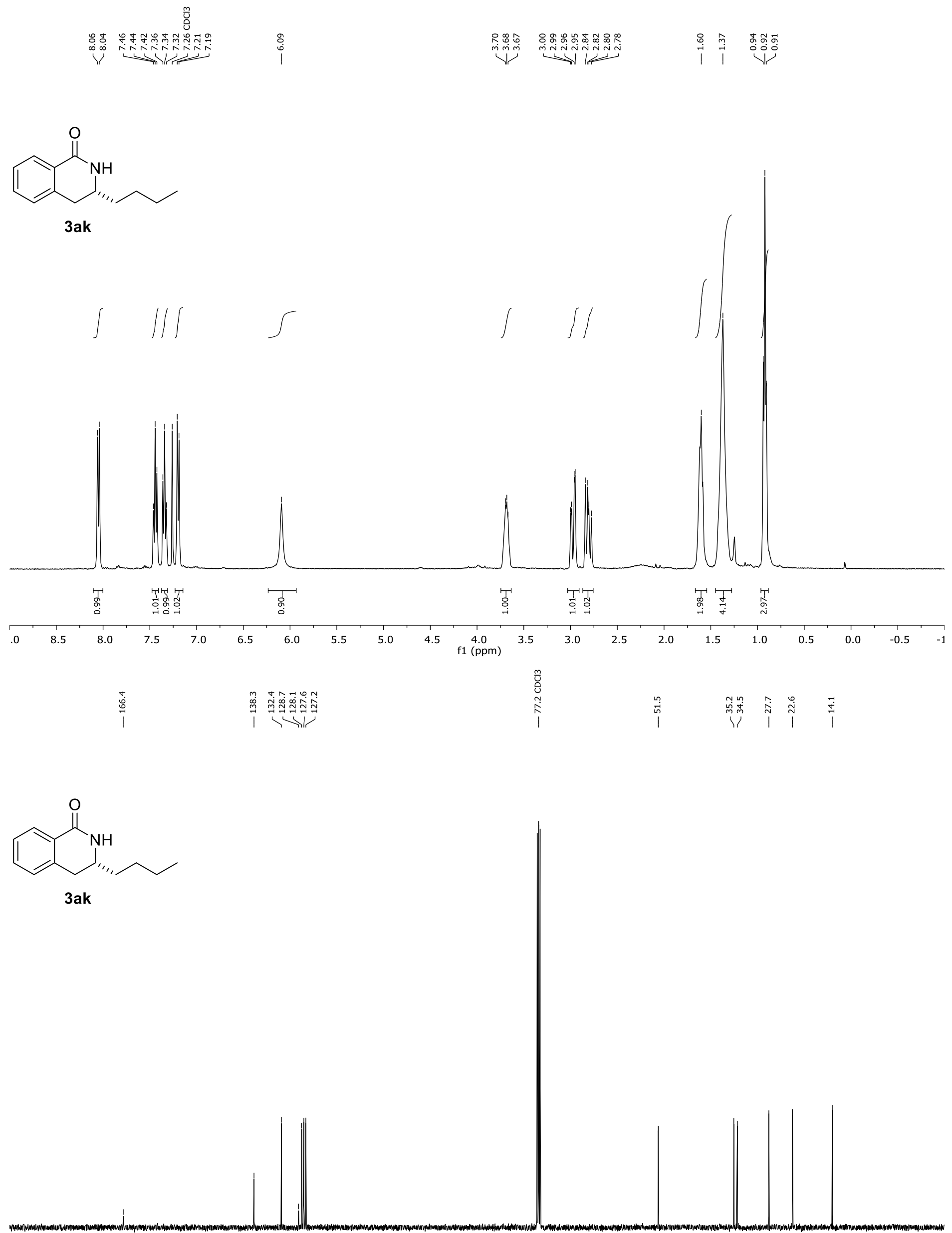


<smiles>CCCCCC[C@@H]1Cc2ccccc2C(=O)N1</smiles>

3al
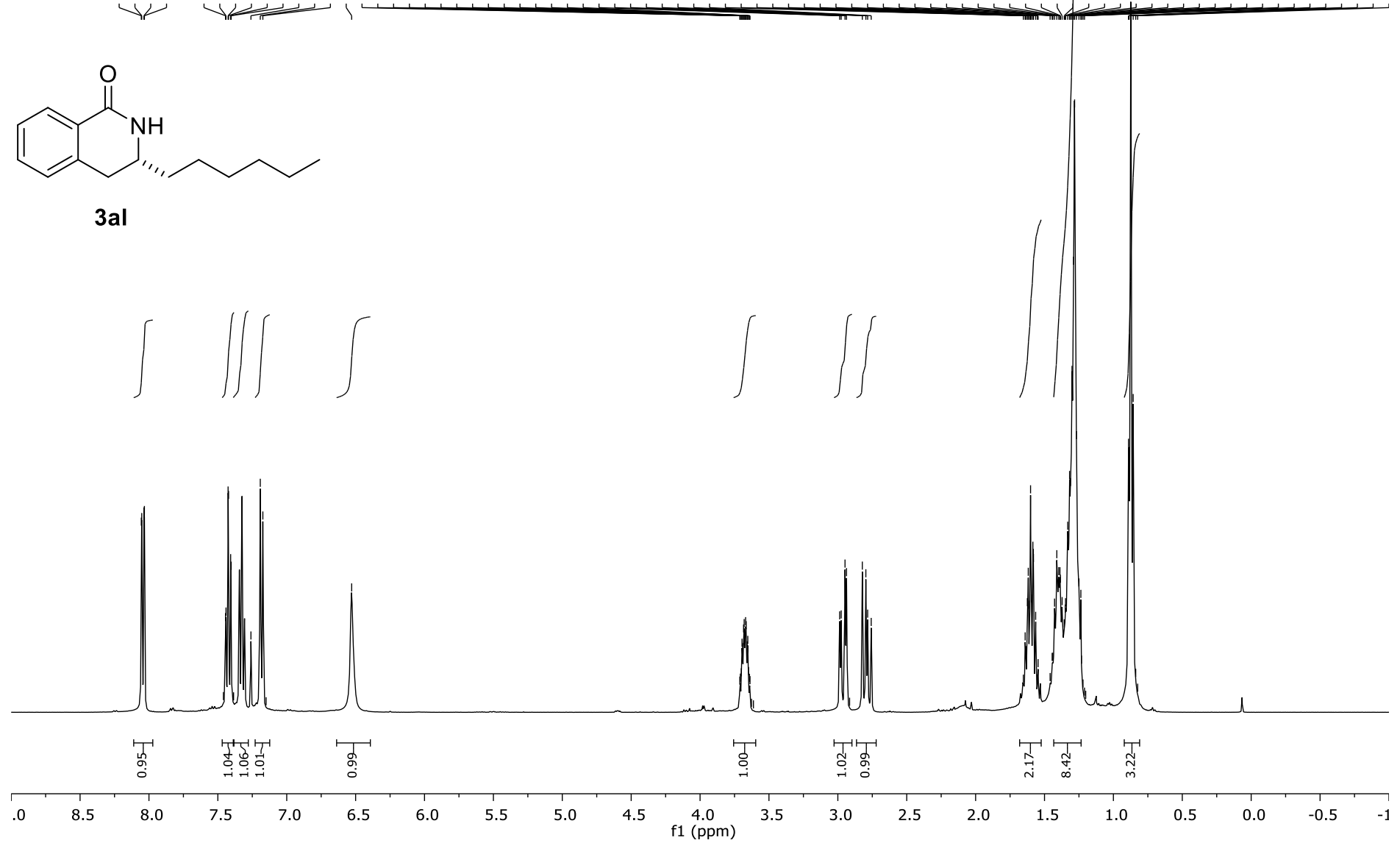

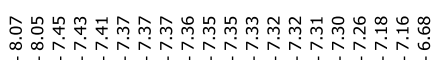

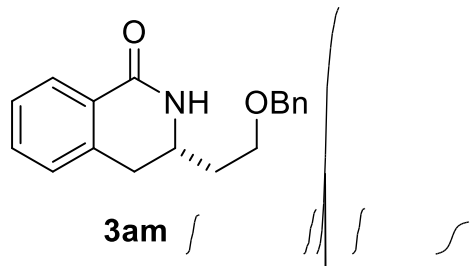<smiles>CCCCCC</smiles><smiles>C1=CC2CC1C2</smiles>
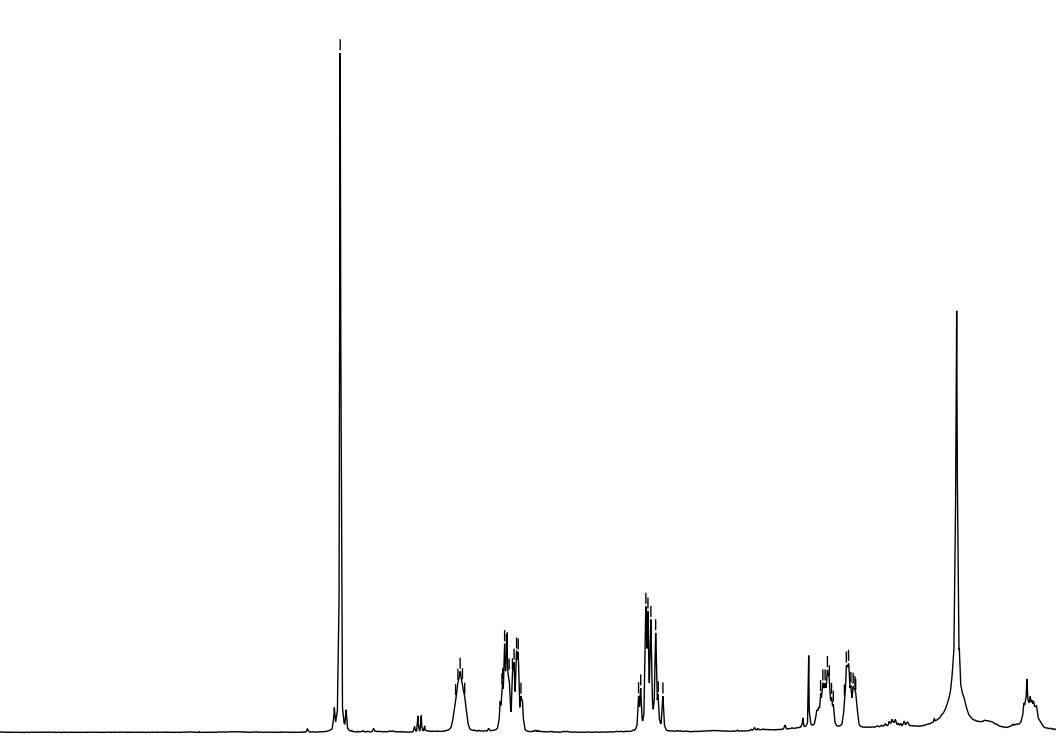

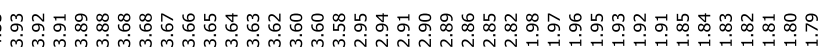

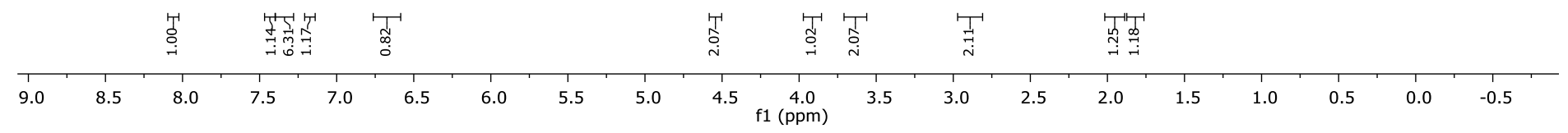


<smiles>O=C1NC(CCOCc2ccccc2)Cc2ccccc21</smiles>

3 am

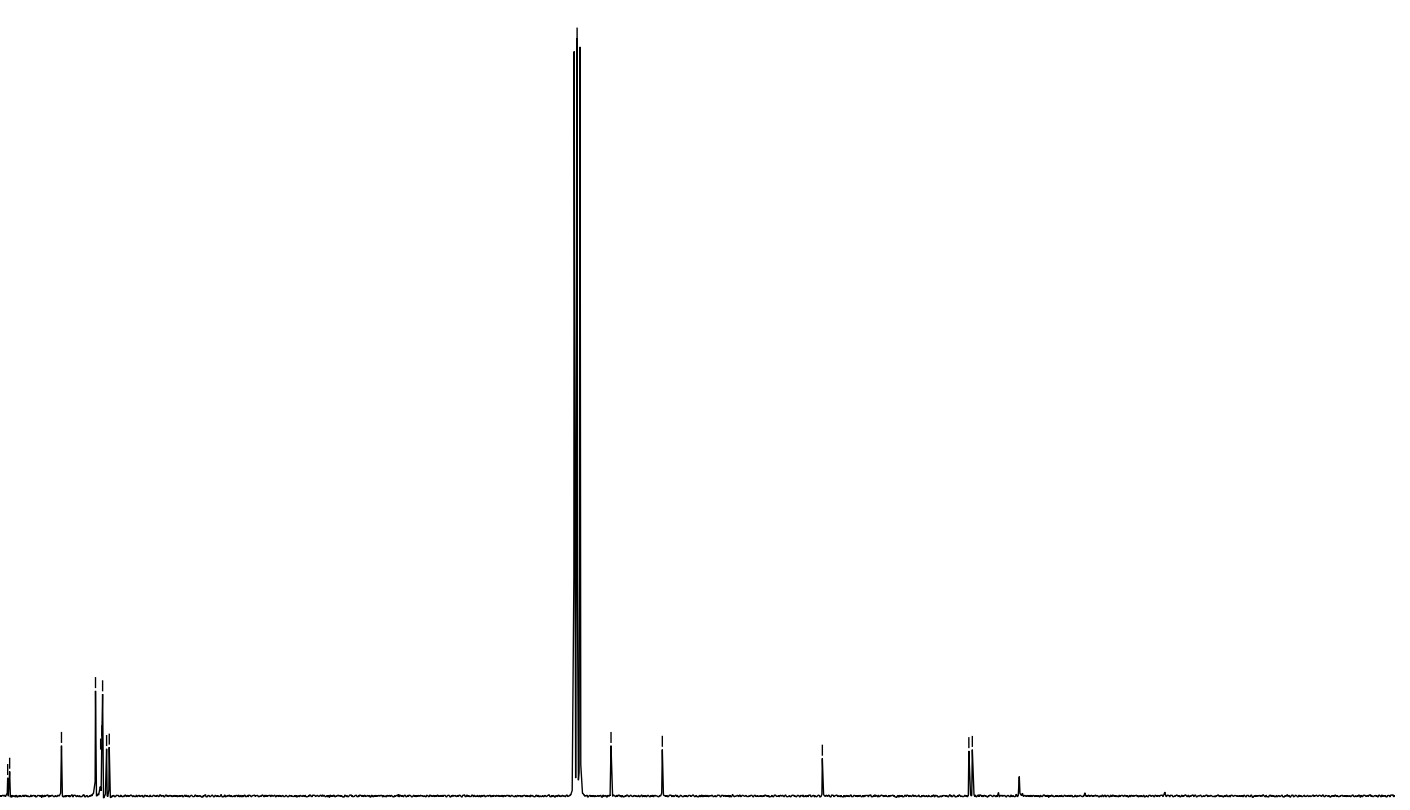

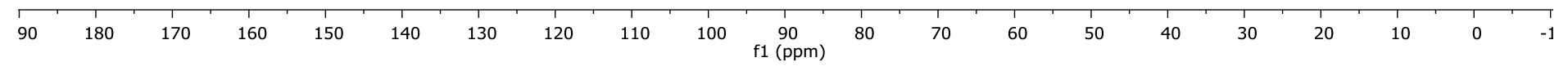

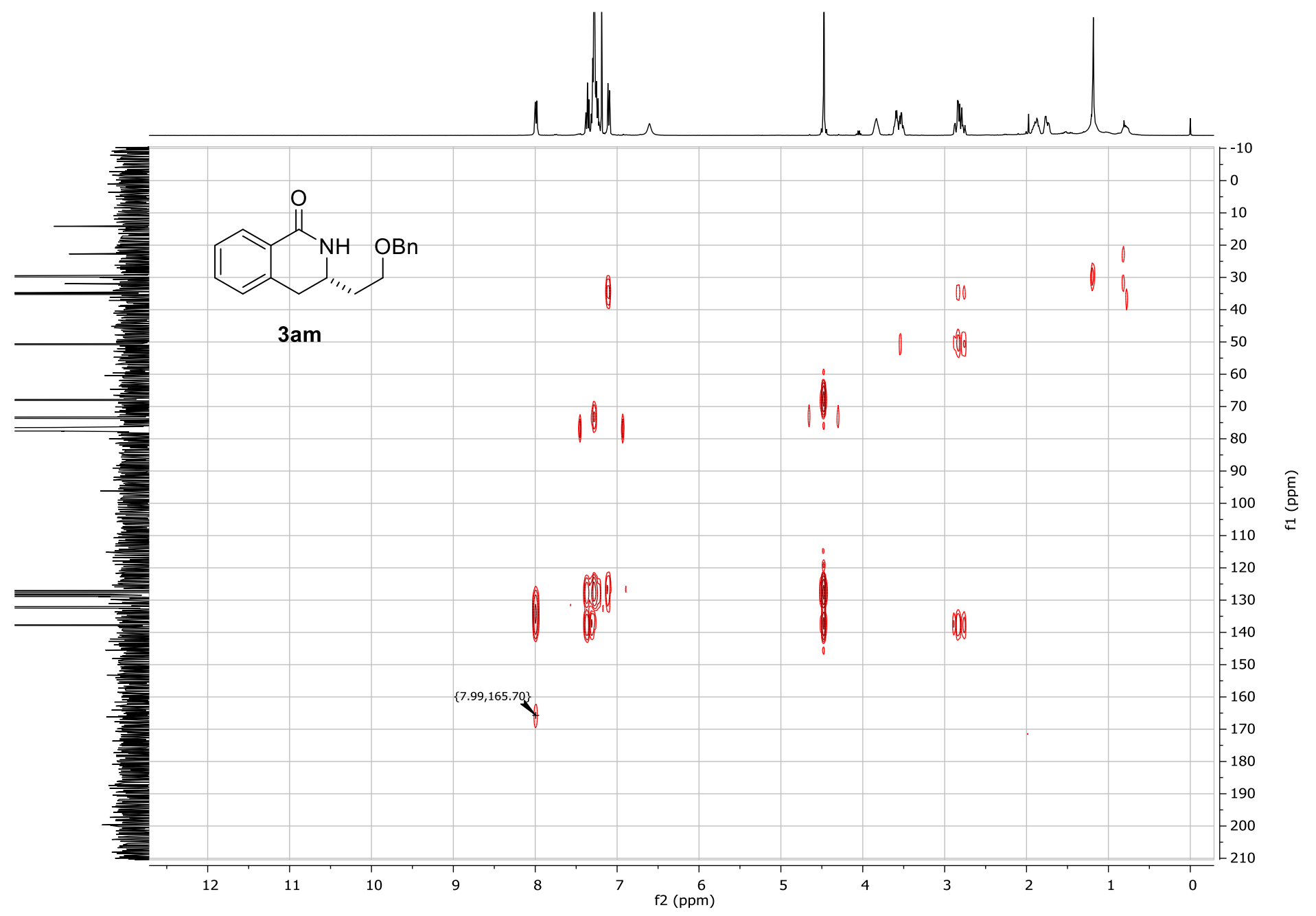

S73 
葛

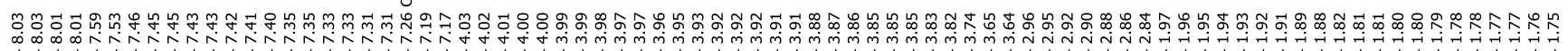<smiles>O=C1N[C@H](CCO)Cc2ccccc21</smiles>

3an
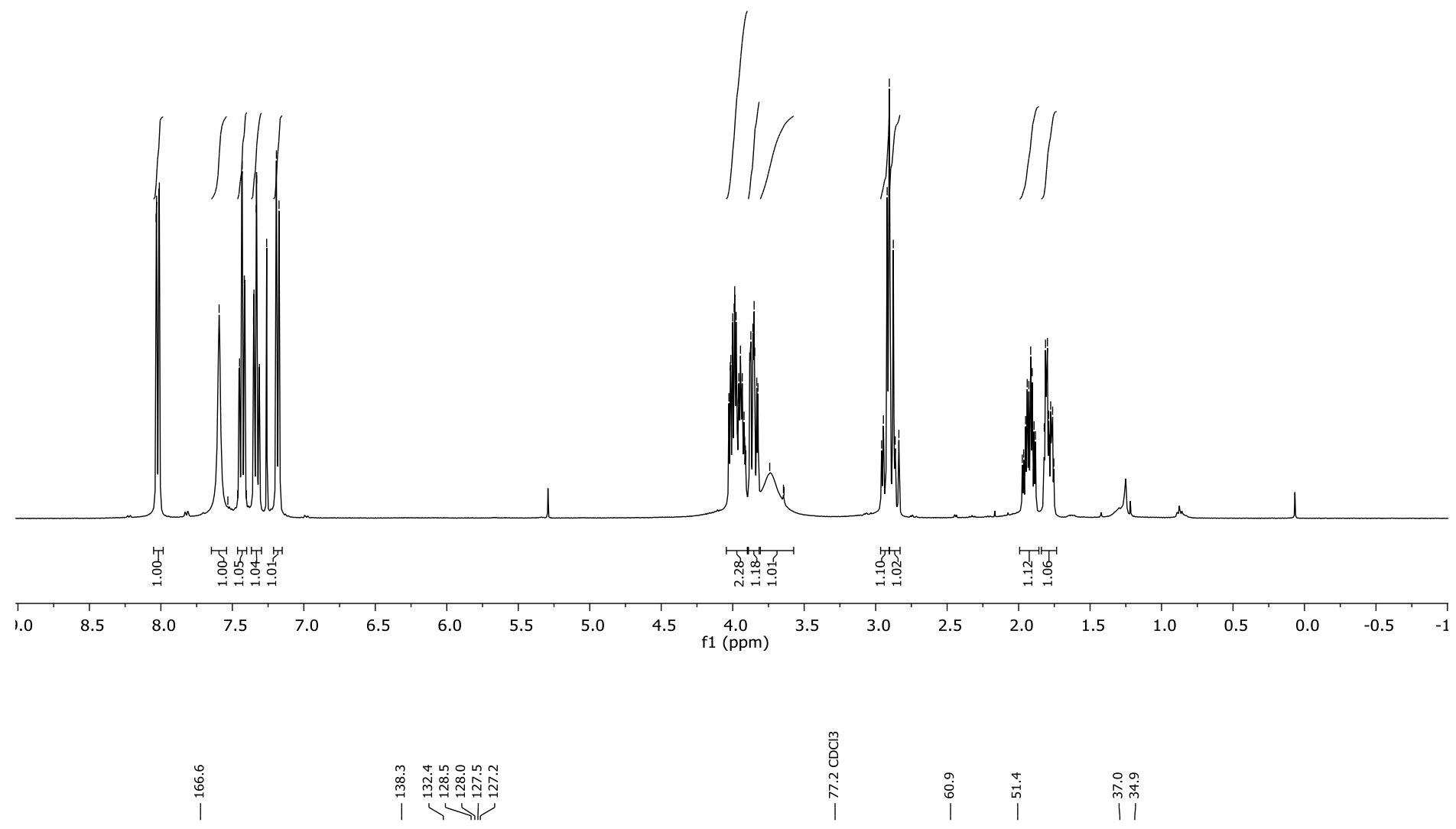<smiles>O=C1N[C@H](CCO)Cc2ccccc21</smiles>
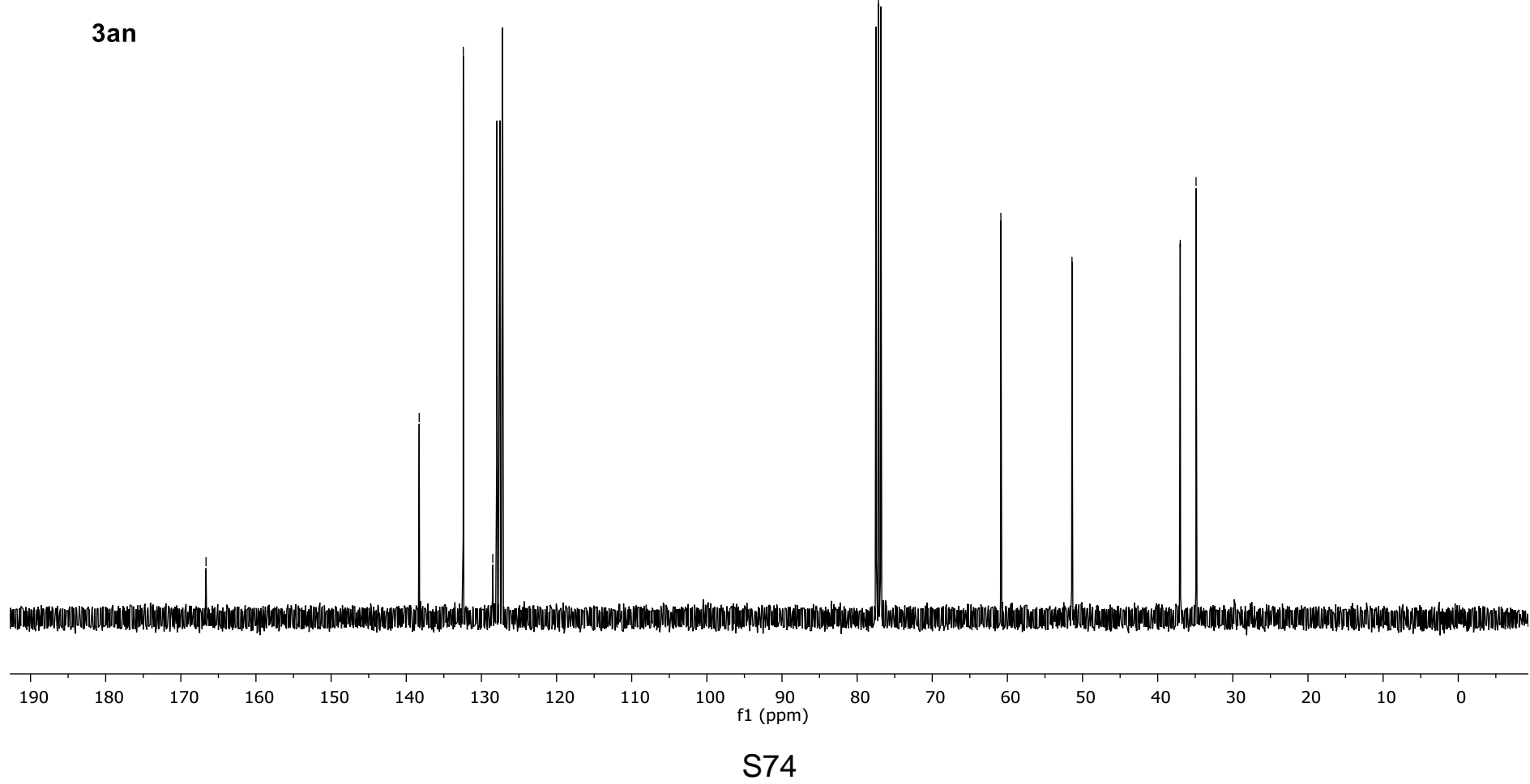

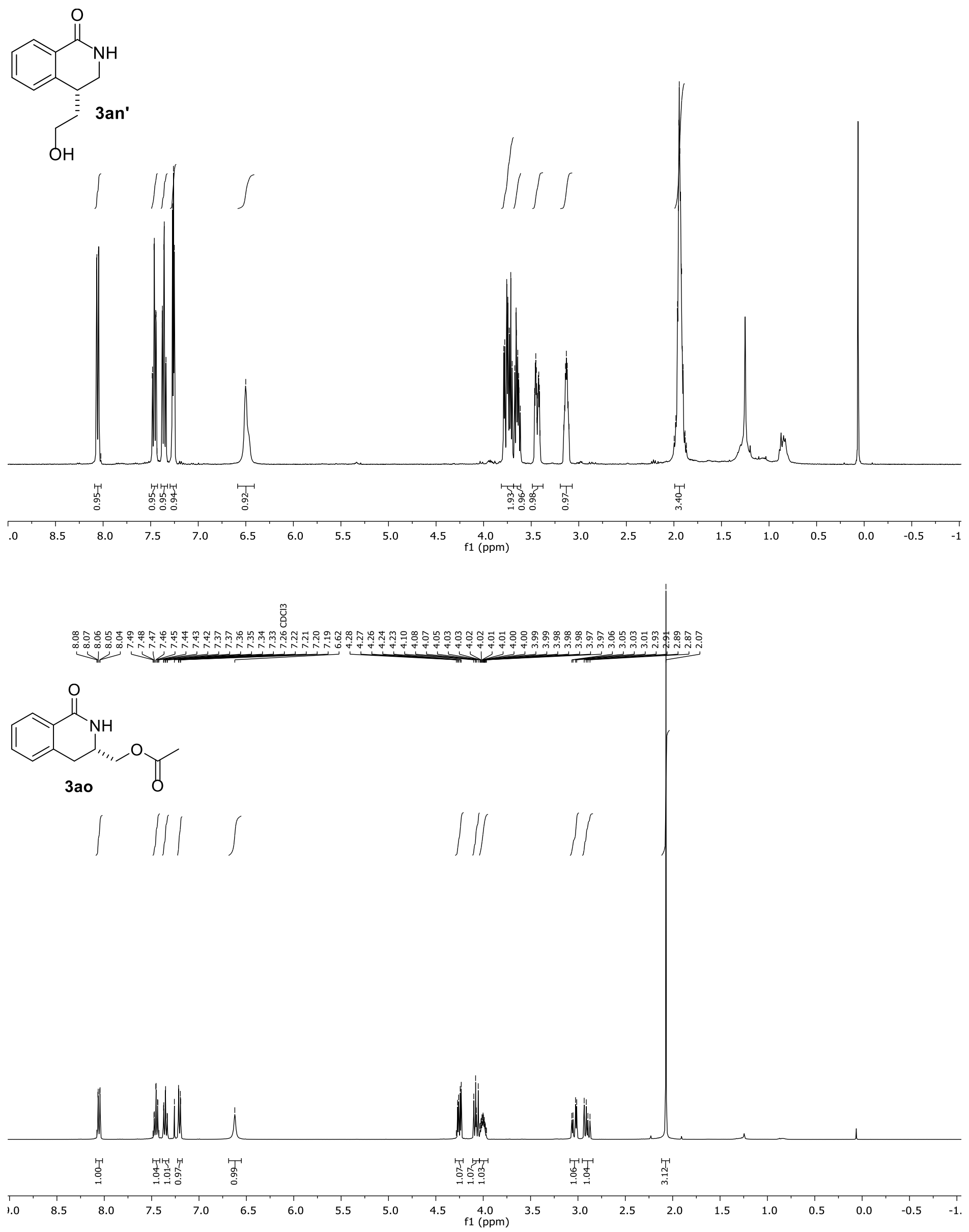


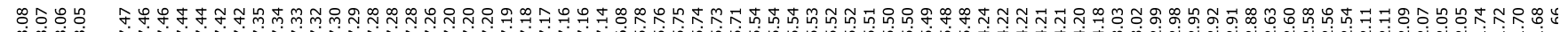

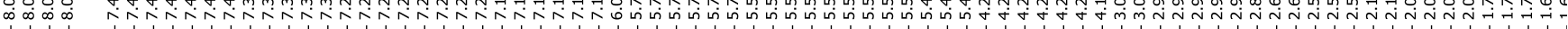<smiles>O=C1N[C@H](/C=C/CCCc2ccccc2)Cc2ccccc21</smiles>

3ap

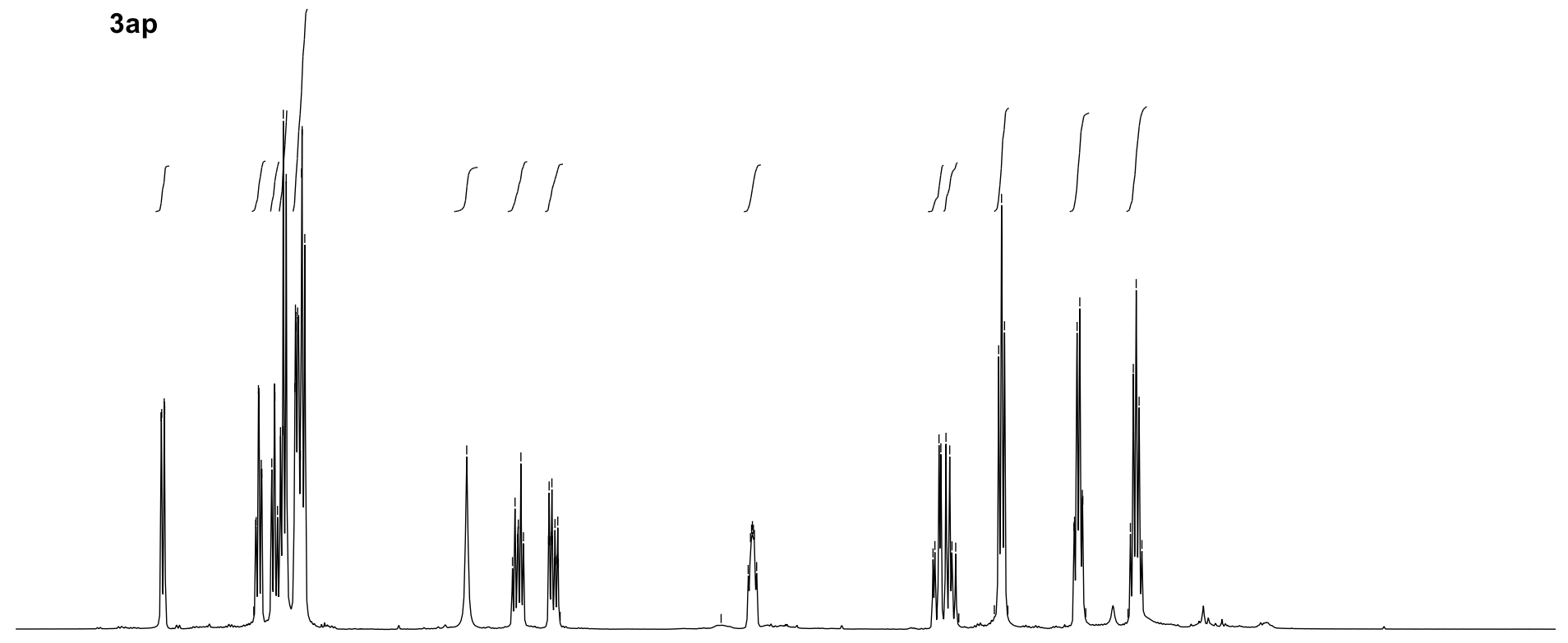

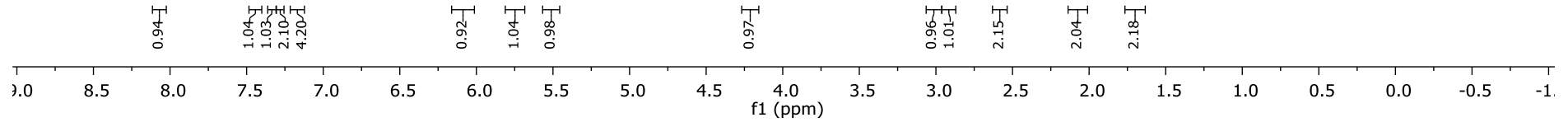

\section{$\underbrace{1}$}
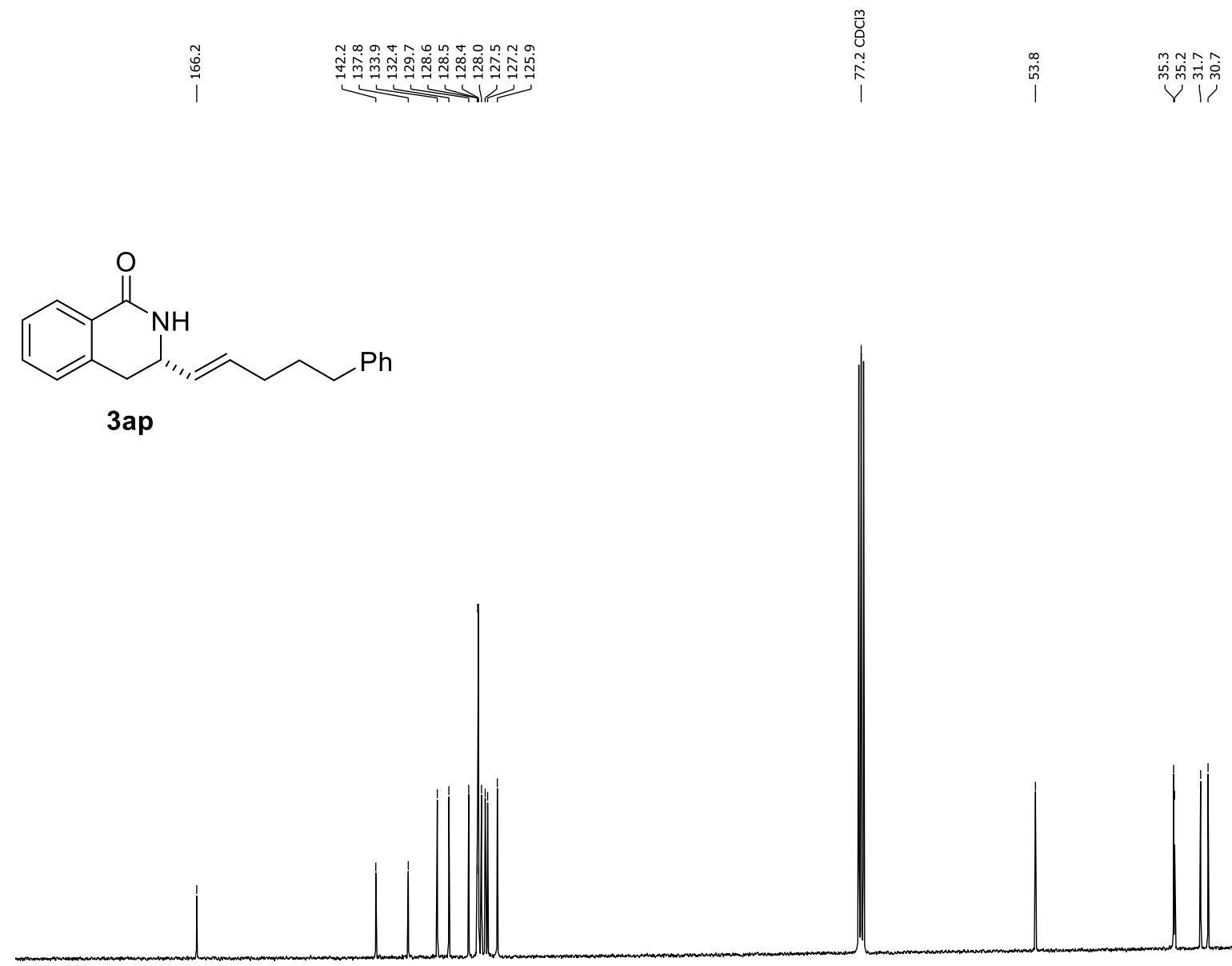$$
90
$$ 

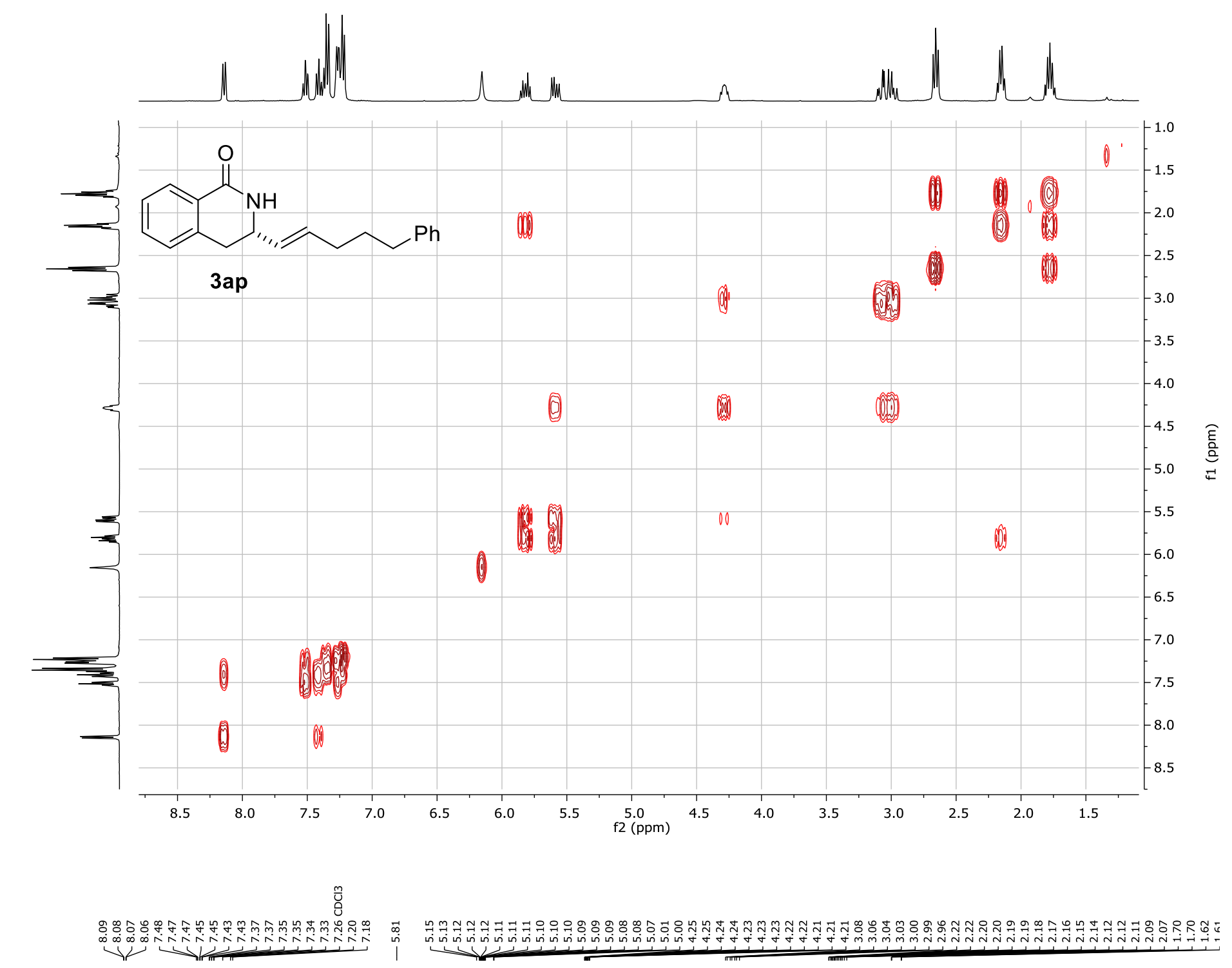<smiles>C=C(CCC=C(C)C)[C@@H]1Cc2ccccc2C(=O)N1</smiles>

$\int\|\| \quad \int \|$

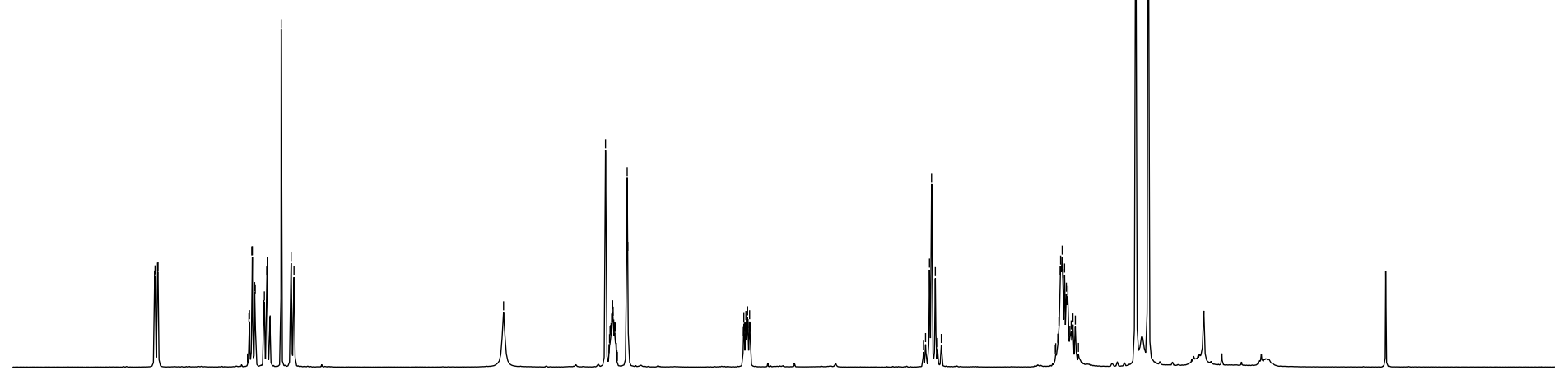

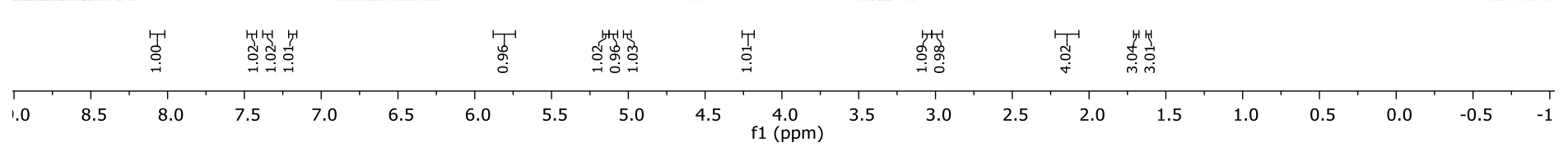




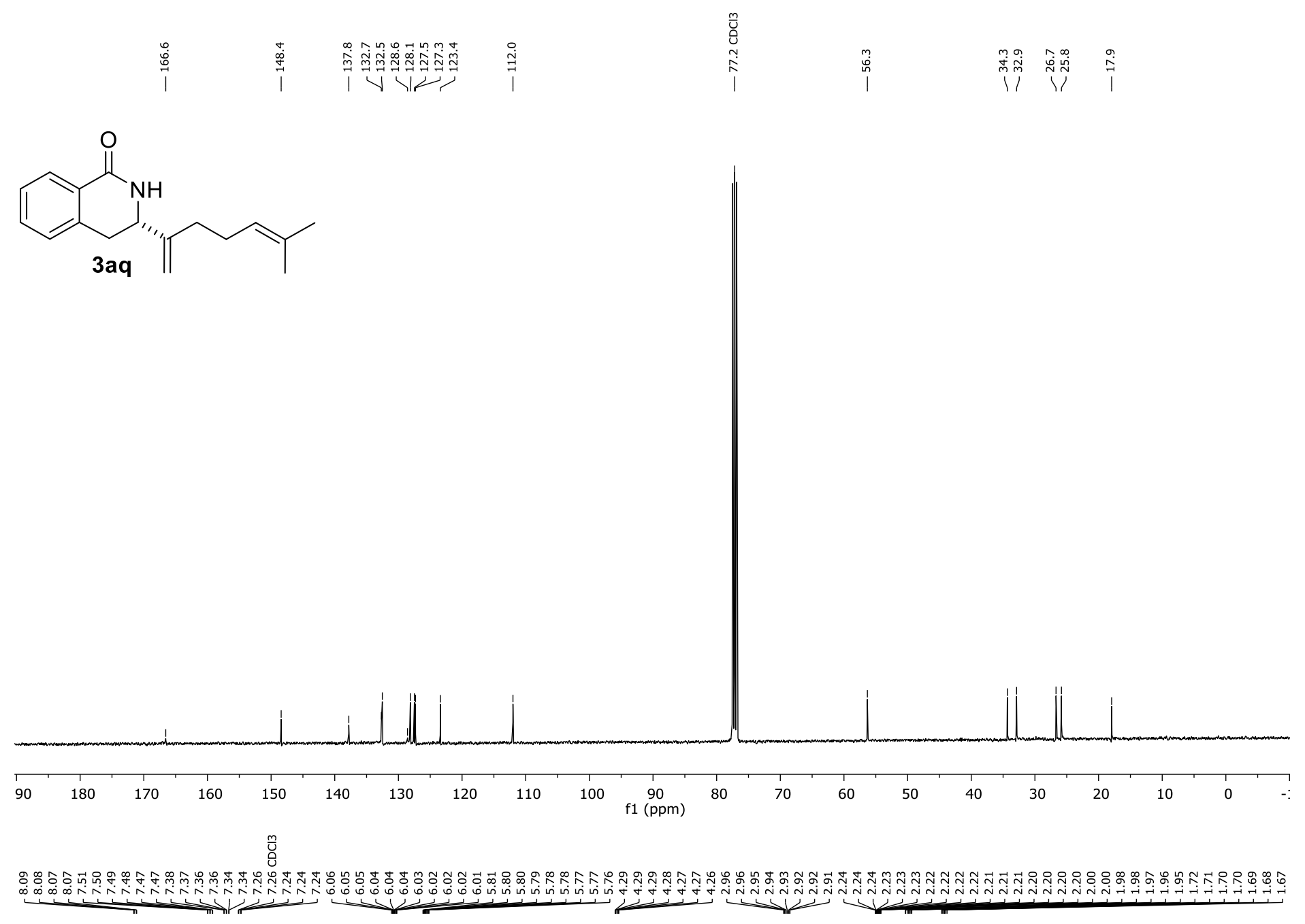<smiles>O=C1N[C@H]2C=CCC[C@H]2c2ccccc21</smiles>
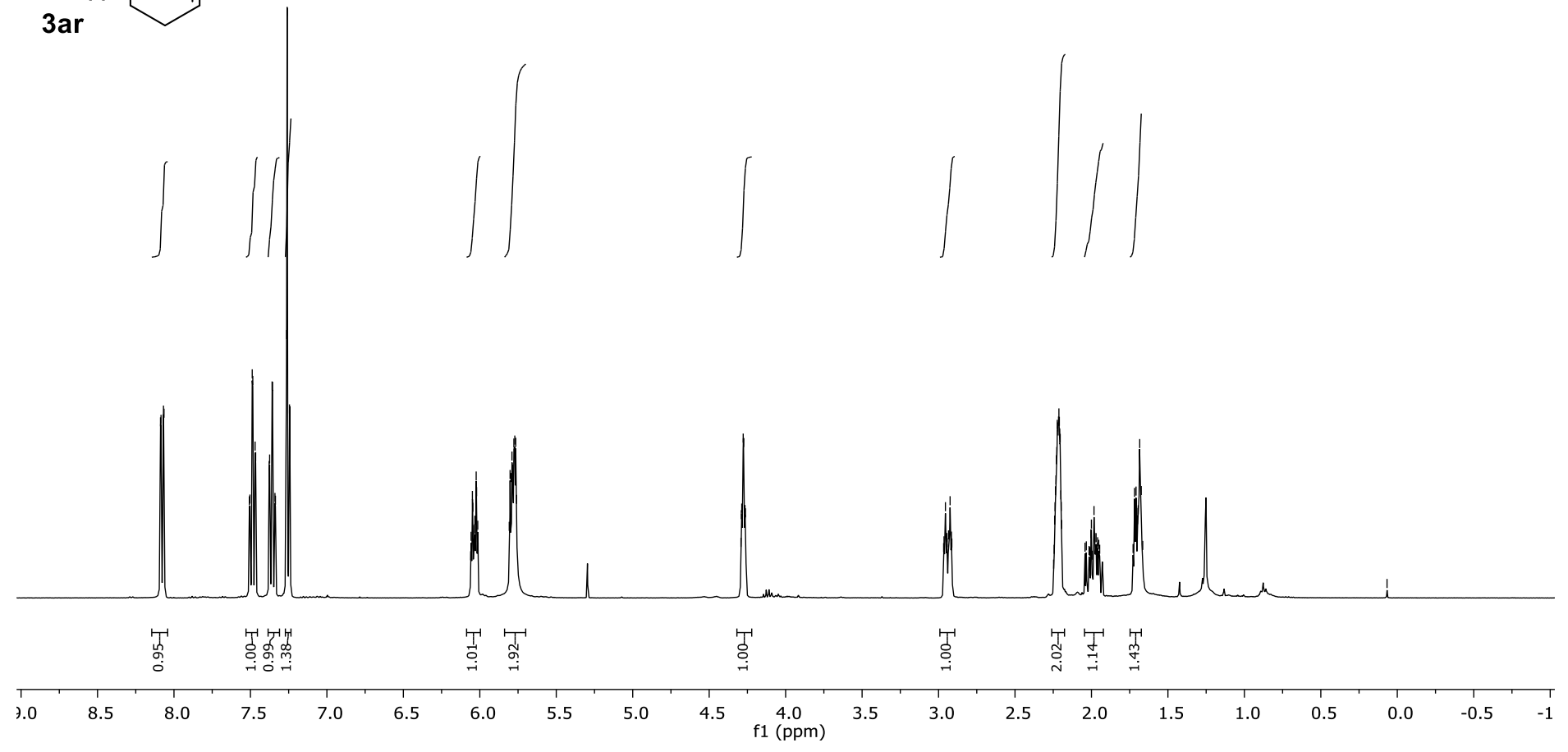


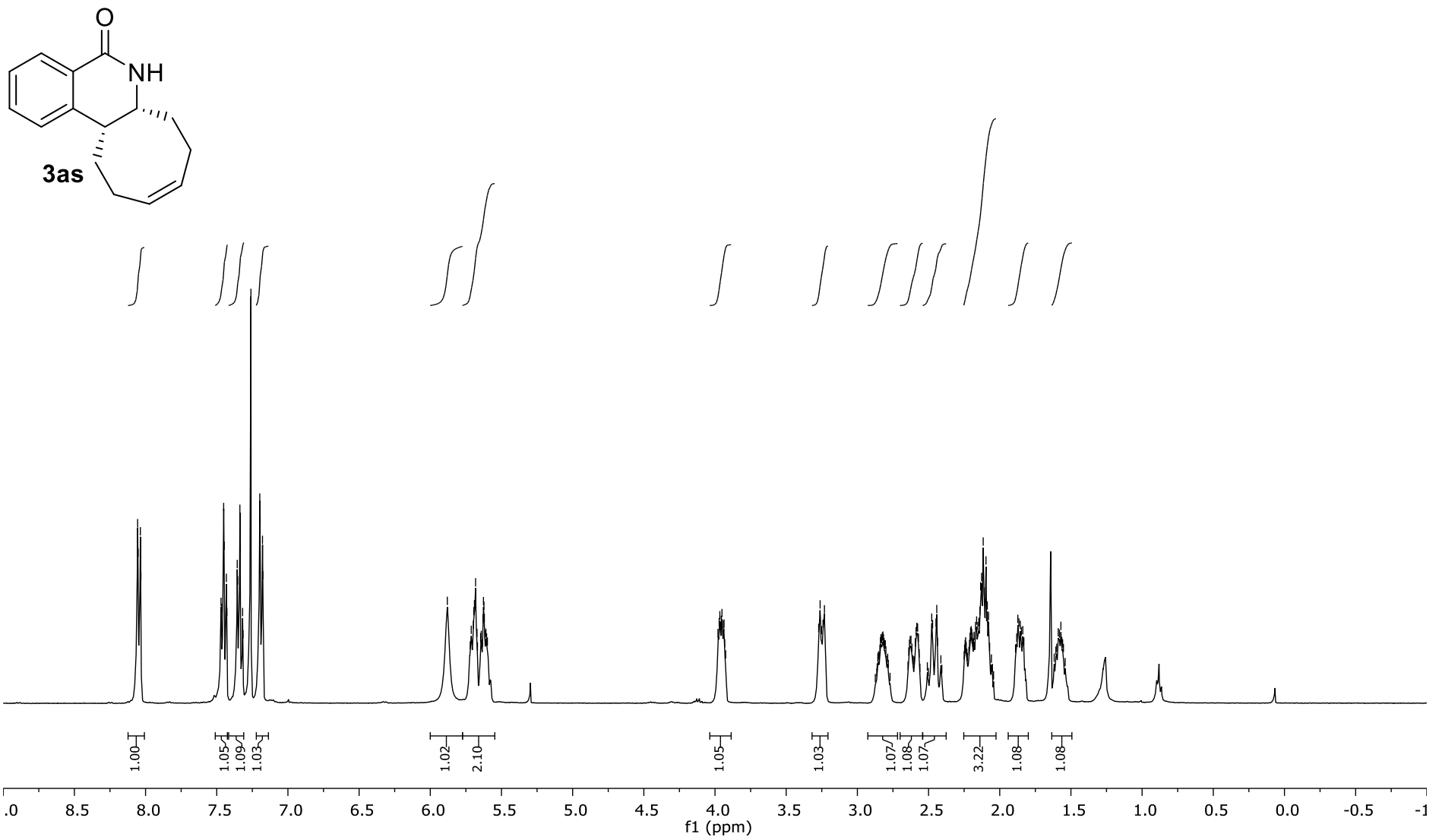

荌

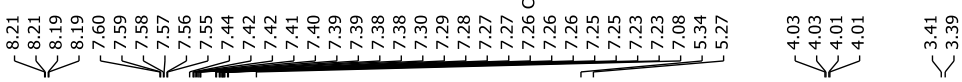<smiles>O=C1N[C@H](Cc2ccccc2)[C@H]2Cc3ccccc3[C@H]12</smiles>

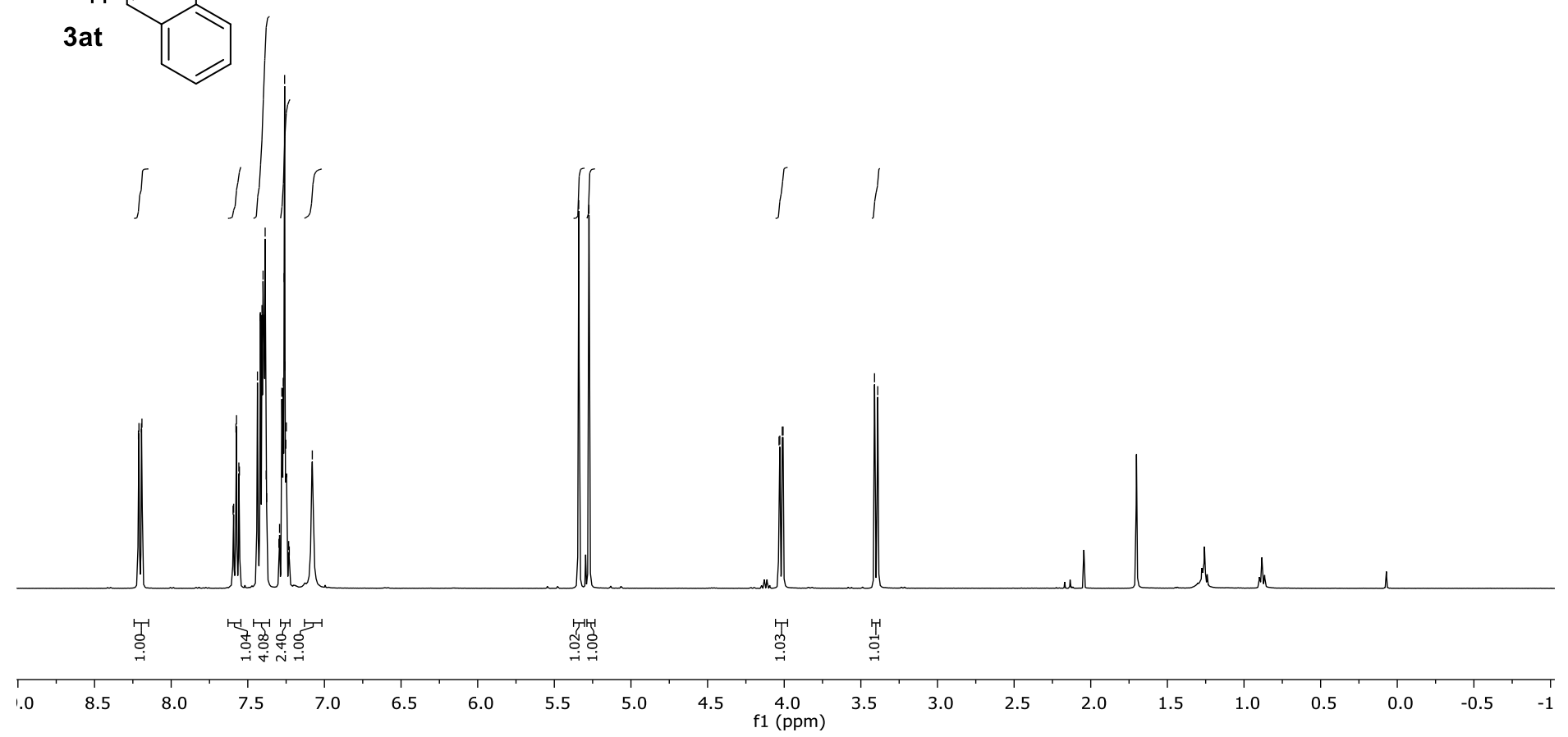



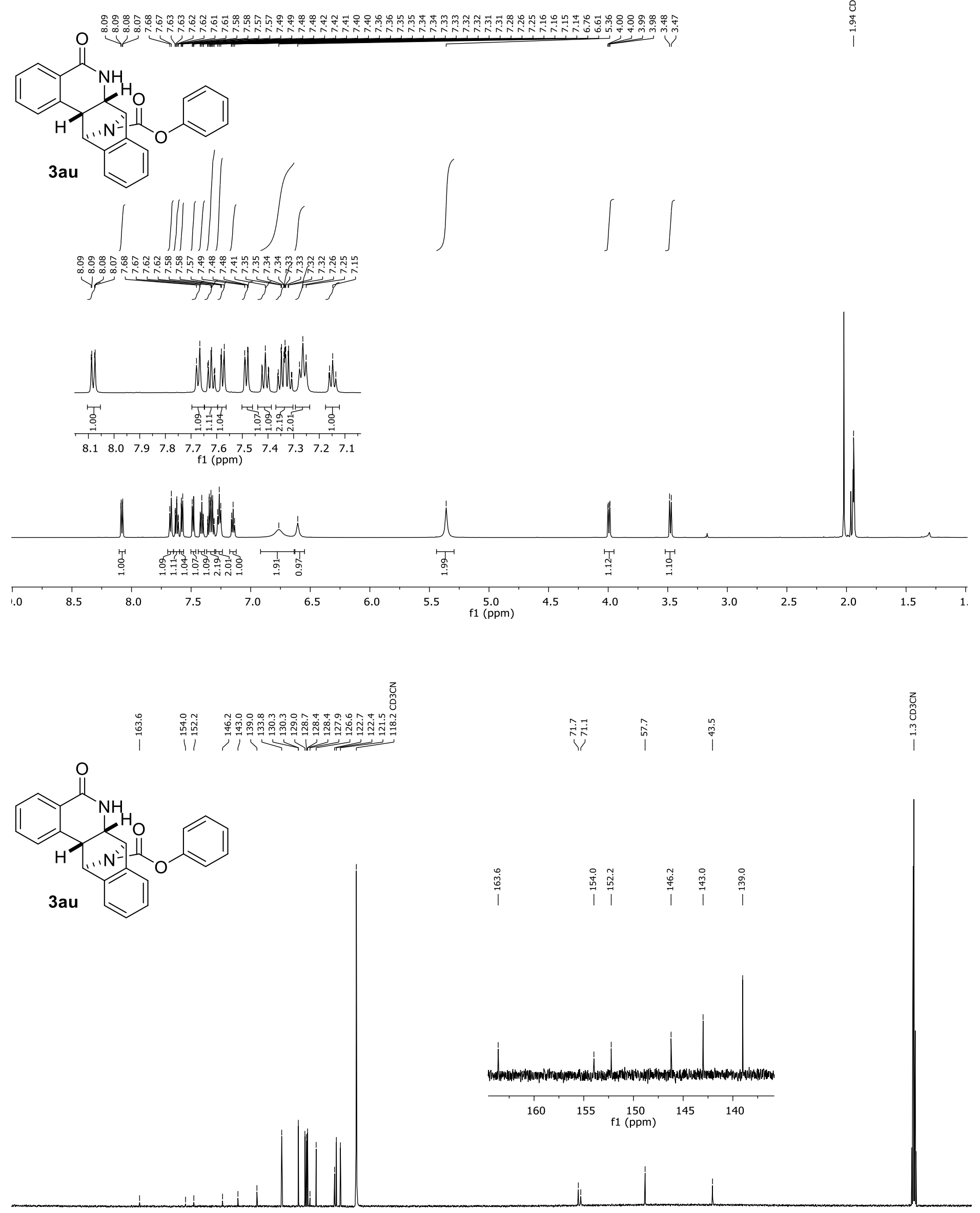

\begin{tabular}{|c|c|c|c|c|c|c|c|}
\hline 180 & 170 & 160 & 150 & 140 & 130 & 120 & 110 \\
\hline
\end{tabular}

